\title{
Association Genetics and Local Adaptation of Populus trichocarpa Torr. \& Gray
}

\author{
Hari Bahadur Chhetri \\ West Virginia University, hchhetri@mix.wvu.edu
}

Follow this and additional works at: https://researchrepository.wvu.edu/etd

Part of the Computational Biology Commons, Genetics Commons, Genomics Commons, and the Molecular Genetics Commons

\section{Recommended Citation}

Chhetri, Hari Bahadur, "Association Genetics and Local Adaptation of Populus trichocarpa Torr. \& Gray" (2019). Graduate Theses, Dissertations, and Problem Reports. 7478.

https://researchrepository.wvu.edu/etd/7478

This Dissertation is protected by copyright and/or related rights. It has been brought to you by the The Research Repository @ WVU with permission from the rights-holder(s). You are free to use this Dissertation in any way that is permitted by the copyright and related rights legislation that applies to your use. For other uses you must obtain permission from the rights-holder(s) directly, unless additional rights are indicated by a Creative Commons license in the record and/ or on the work itself. This Dissertation has been accepted for inclusion in WVU Graduate Theses, Dissertations, and Problem Reports collection by an authorized administrator of The Research Repository @ WVU.

For more information, please contact researchrepository@mail.wvu.edu. 
TORR. \& GRAY

Hari Bahadur Chhetri

\begin{abstract}
Dissertation submitted to the Eberly College of Arts and Sciences

at West Virginia University

in partial fulfillment of the requirements for the degree of
\end{abstract}

Doctor of Philosophy in

Biology

Stephen P. DiFazio, Ph.D., Chair
Donna Ford-Werntz, Ph.D.
Jennifer Hawkins, Ph.D.
Richard Thomas, Ph.D.
Vagner Benedito, Ph.D.
Department of Biology

Morgantown, West Virginia 2019

Keywords: Quantitative traits, GWAS, heritability, redundancy analysis, local adaptation, outlier loci, genes, expression networks

Copyright 2019 Hari Bahadur Chhetri 


\section{Abstract \\ Association genetics and local adaptation of Populus trichocarpa Torr. \& Gray}

Hari Bahadur Chhetri

A major goal in plant science is overcoming the recalcitrance of plant biomass to cellulose extraction, to enable efficient production of cellulosic biofuel. We have started to understand the genetic basis of some important traits such as cell wall chemistry, but we do not know anything about the key structural and functional traits such as wood anatomy that greatly affect plant biomass recalcitrance. Furthermore, biofuel feedstocks have to be adapted to varied environmental conditions to ensure high productivity in plantations, but little is known about the molecular mechanisms underlying local adaptation. With the advancement in sequencing and genotyping technologies, association genetics has emerged as a powerful approach for unraveling complex traits in plants, thereby linking the natural variation present in the phenotype with the underlying genotype. Furthermore, the integration of phenotypic, genomic and environmental data has great premise for understanding plant adaptation in the face of climate change.

Because of its rapid growth, hybrid vigor, broad geographic distribution, transformation potential, and the availability of tremendous genetic resources and wide phenotypic variation, Populus is a highly desirable genus for biofuel production and other wood products. My dissertation research uses an association genetics approach focused on important anatomical, morphological and physiological traits to address three key questions: (1) What genetic mechanisms underlie variation in morphological and physiological traits in P. trichocarpa? (2) What are the factors affecting local adaptation in P. trichocarpa and what is the relative contribution of climate and geography variables to population structure? (3) What genes or genomic regions are associated with variation in important functional and structural traits that can be targeted to enhance productivity and reduce recalcitrance of woody bioenergy feedstocks?

My research will enhance understanding of the biology of Populus trichocarpa by determining the genetic basis of key agronomic traits such as vessel size and density, leaf area, and stomatal density that affect overall performance under field conditions using genome-wide association study (GWAS). Understanding the genetic basis of these traits is key for developing Populus as a biomass feedstock for biofuel production. Furthermore, morphological and structural traits are often tightly correlated with physiological performance. Therefore, another aspect of this study is to unravel the genetic basis of key physiological traits such as leaf chlorophyll content, carbon isotope composition and leaf water potential, and their correlation with morphological traits. This will aid in better understanding of stress tolerance and the overall biology of this species. Furthermore, by performing these studies in plantations that are clonally replicated in three environments, I evaluated the robustness of the associations. Using genotype environment association (GEA) and redundancy analysis (RDA) I identified loci conferring local adaptation in $P$. trichocarpa. Moreover, with RDA analysis I determined the relative contribution of climate and geography in neutral population structure. Similarly, I determined the relative contribution of genomic, climate and geography data in explaining phenotypic variation. A long-term goal of the project is to develop a selection model based on comprehensive genetic and phenotypic information so that the genome enabled breeding value can be estimated. This will enhance the efficiency of Populus breeding programs by shortening the breeding cycle and improving the accuracy of selection. This will aid in developing genetically improved trees with high biomass production and reduced recalcitrance to cellulose extraction, thereby furthering the development of the lignocellulosic biofuels industry. 


\section{Dedication}

To my Parents 


\section{Acknowledgements}

First of all, I thank my advisor Dr. Stephen P. DiFazio for providing me the opportunity to work in his lab, and for all the guidance and help he provided me through-out this entire research journey. I am who I am today because of him. I thank my committee members Dr. Vagner Benedito, Dr. Donna FordWerntz, Dr. Jennifer Hawkins and Dr. Richard Thomas for their valuable comments and suggestions during our meetings.

I thank former postdocs in the lab, Dr. Alejandro Riveros Walker, Dr. Danielle Ellis, Dr. Luke Evans, Dr. Gancho Slavov and the current postdoc Dr. David Macaya-Sanz for their help in fieldwork, statistical analysis and programming. I thank Dr. Jorge Flores, professor at the Department of Biology (now retired) for letting me use his histology facility. I have spent countless hours staring at the microscope in your lab to process my wood anatomy samples.

I thank my lab-mates Dr. Brahma Reddy Induri, Dr. Eli Rodgers-Melnick, Sandy Simon and Ran Zhou for their help in fieldwork and all the great interactions I have had with them over the years.

I thank several undergrads that helped me for collecting data in the field and in the lab for my research - Amanda Emahizer, Luke Stover, Jacob Miller, Patrick Whitehouse, Hoff Lindberg and Savanna Plombon. I thank Sunita Mahat (graduate intern) for helping me with the image analysis using imageJ and matlab.

I thank Dr. Wellington Muchero, Dr. Jay Chen, Dr. Jerry Tuskan, Dr. Jessy Labbe, Lee Gunter, Sarah Jawdy, and Dr. Tony Bryan form Oak-Ridge National Lab for their help in the fieldwork. I thank my teaching mentors Pat Lutsie (now retired), Dr. Katrina Stewart, Dr. Michelle Withers, Dr. Dana Heubert Lima and Dr. Stephanie Young. You have taught me so much to grow as a teacher. I have learned so much from you guys, and you really made the teaching enjoyable.

I cannot thank my wife, Anita enough for her continuous support during the entire period of my graduate studies. She has contributed so much. Thanks for taking care of the family, good food and moral support. Thank you, Elina (daughter) and Elesh (son), you are the joy of my life. At last, but not least, I thank my parents, Krishna and Ganga for all their support and blessings and believing in me. 


\section{Table of contents}

ASSOCIATION GENETICS AND LOCAL ADAPTATION OF POPULUS TRICHOCARPA TORR. \& GRAY

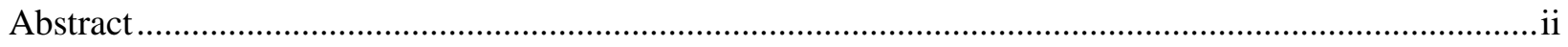

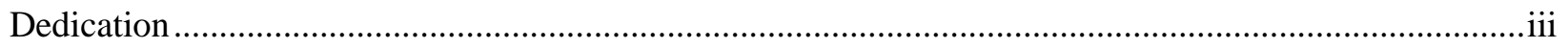

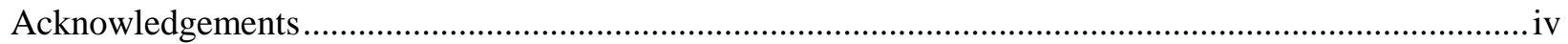

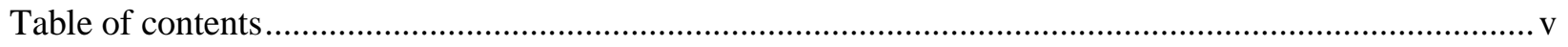

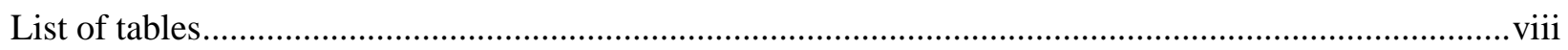

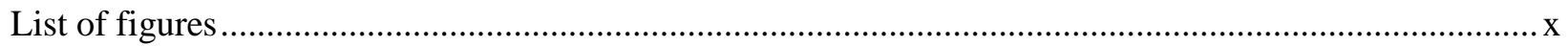

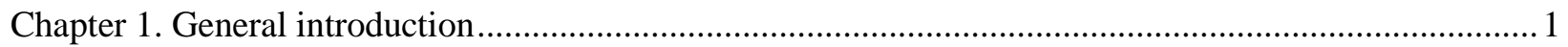

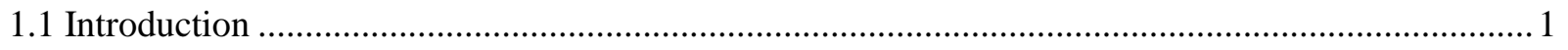

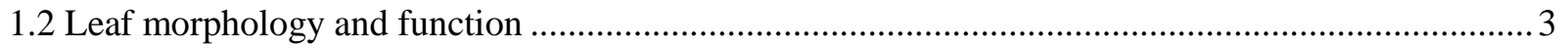

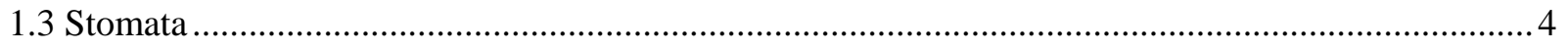

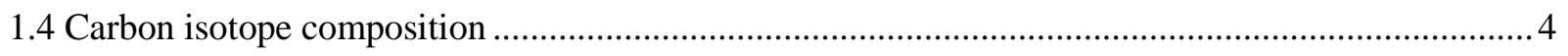

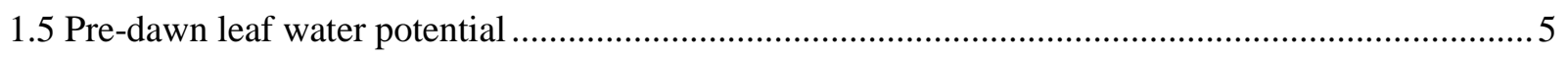

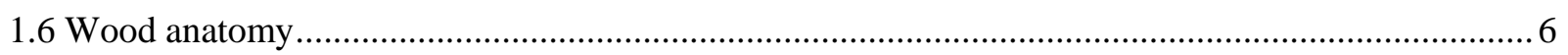

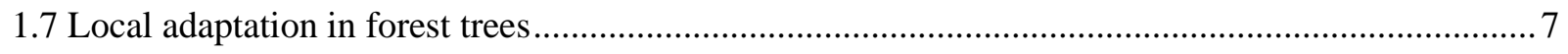

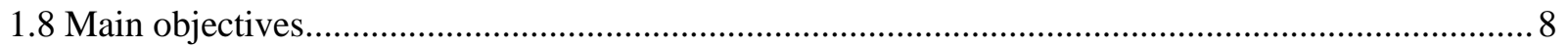

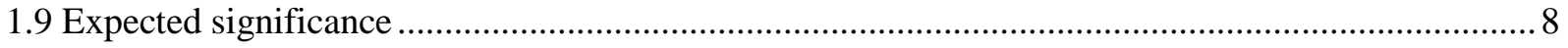

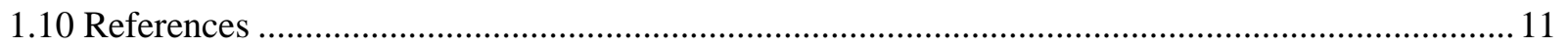

Chapter 2. Multitrit genome-wide association analysis of Populus trichocarpa identifies key polymorphisms controlling morphological and physiological traits....................................................20

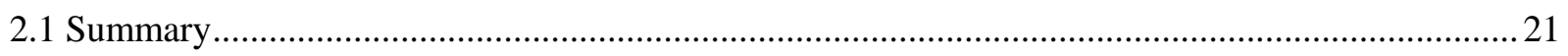

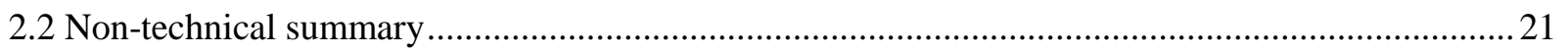

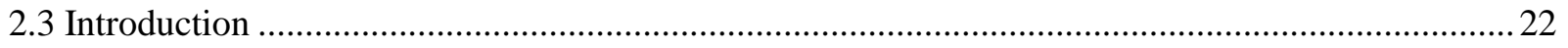

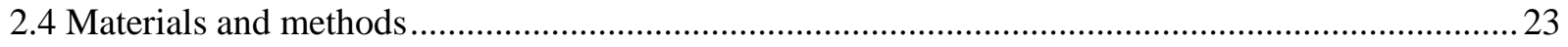

2.4.1 Plantation establishment and phenotyping ....................................................................23

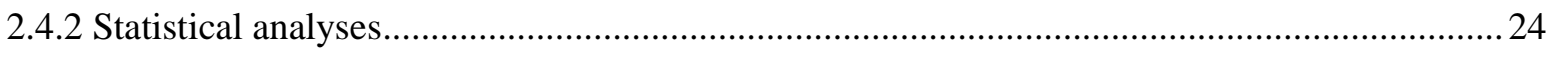

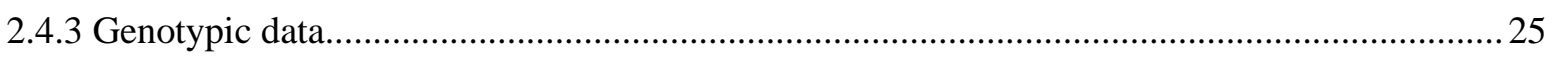

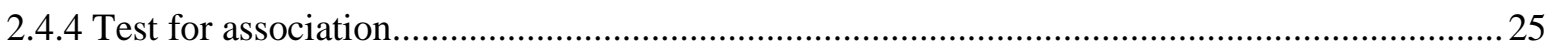

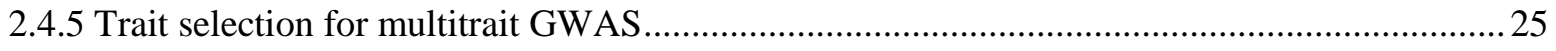

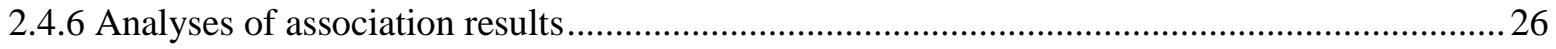

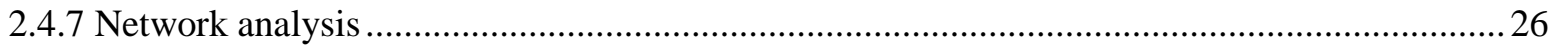

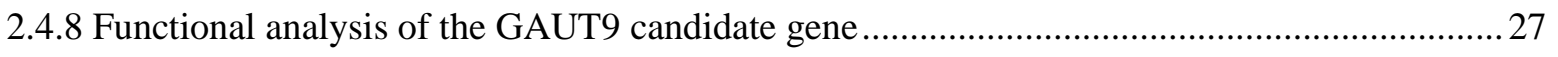

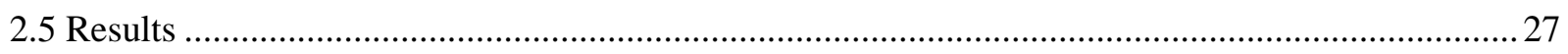

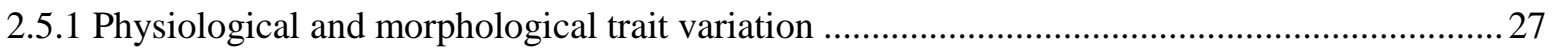




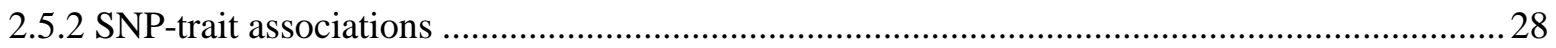

2.5.3 Direct evidence of the role of GAUT9 in determining leaf area in Populus ............................29

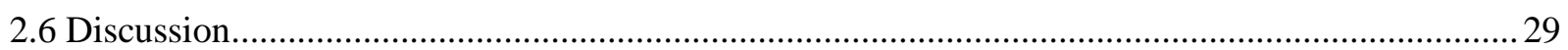

2.6.1 Morphological and physiological trait correlations and influence of geography ..................... 30

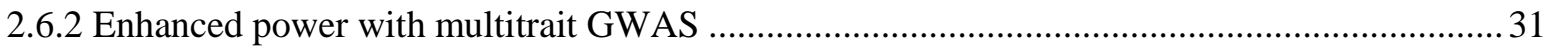

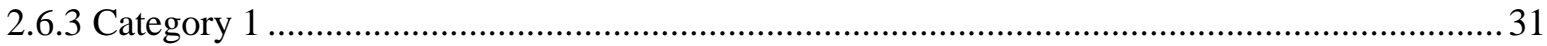

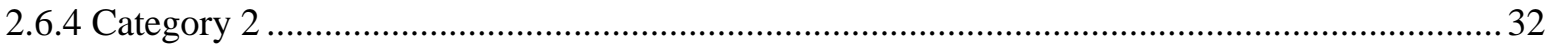

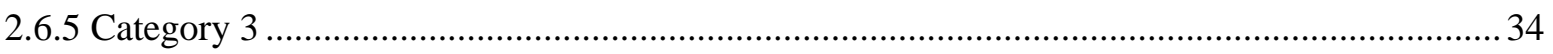

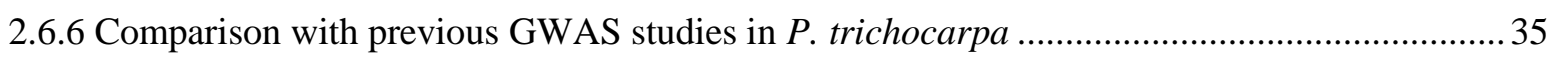

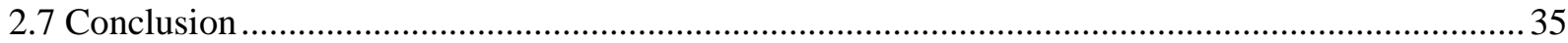

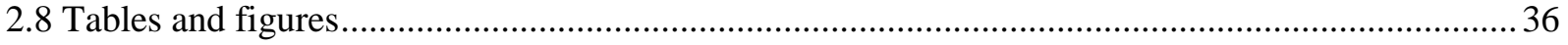

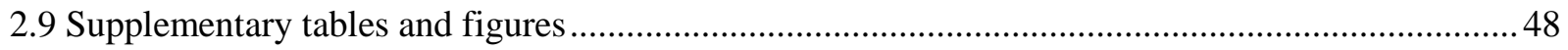

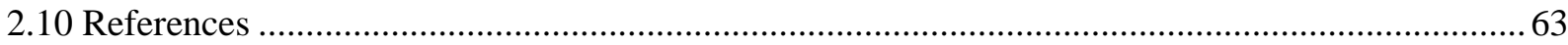

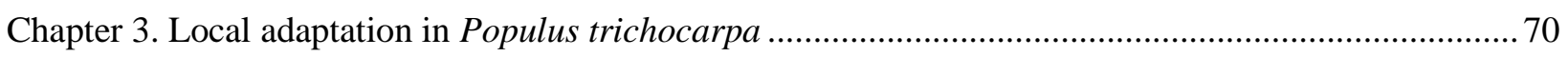

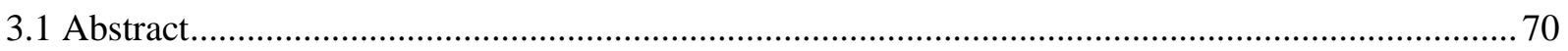

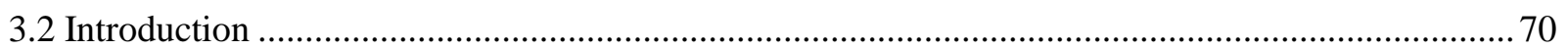

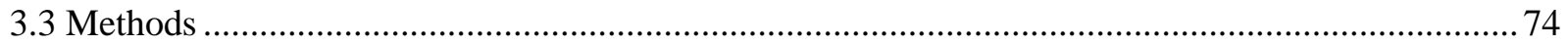

3.3.1 Plantation establishment and sampling …........................................................................ 74

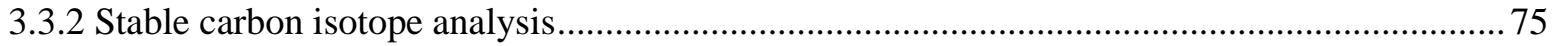

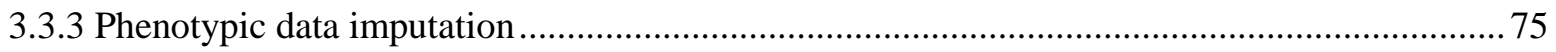

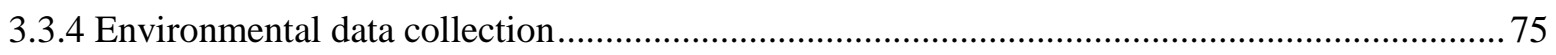

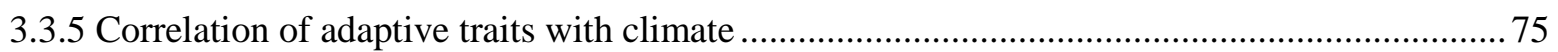

3.3.6 Test for the association of climate variables with the SNPs in the genome ............................. 76

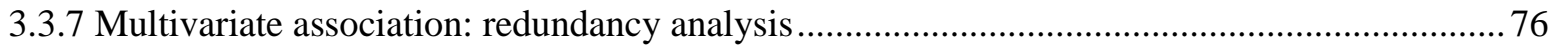

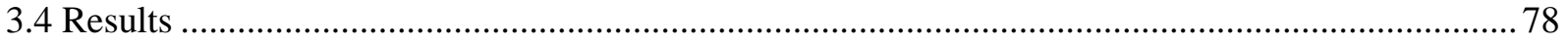

3.4.1 Trait heritabilities and genetic correlation of phenotypes with geography and climate variables

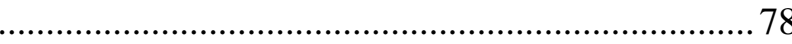

3.4.2 Detection of outlier loci using genotype-environment association (GEA) ........................... 78

3.4.3 Genes identified by genotype-environment association (GEA) …...................................... 79

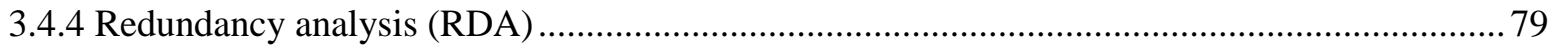

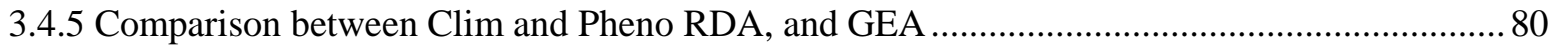

3.4.6 Comparing Pheno RDA model with simple multiple regression models................................ 81

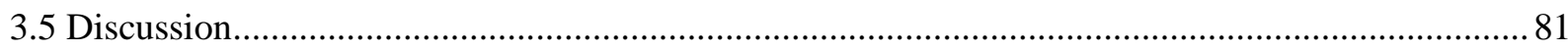

3.5.1 Genetic variation (phenotypic trait) and phenotype environment correlation......................... 81

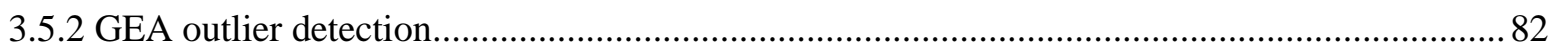

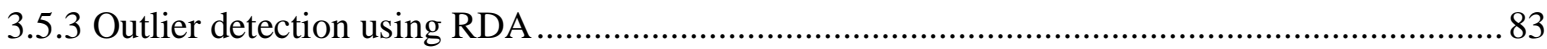

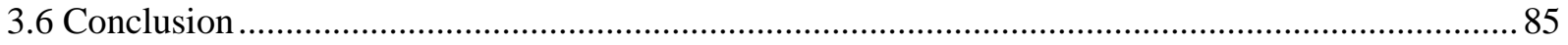




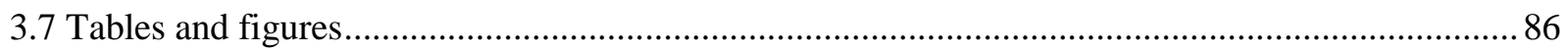

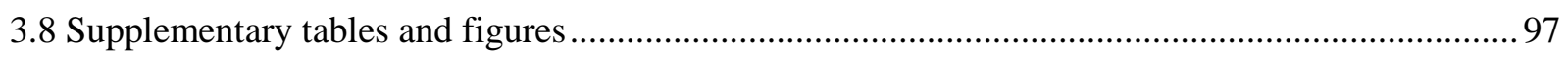

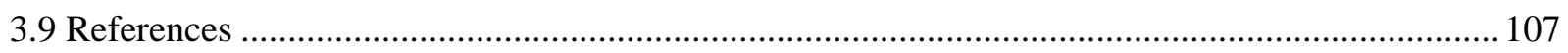

Chapter 4. Genome-wide association study of wood anatomical, wood chemistry and morphological traits

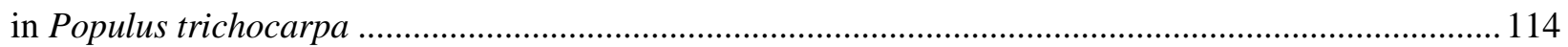

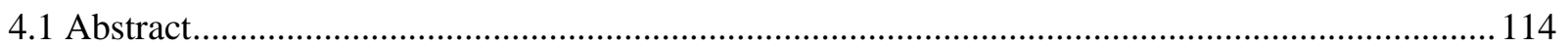

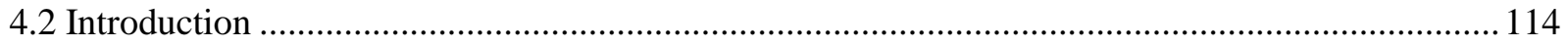

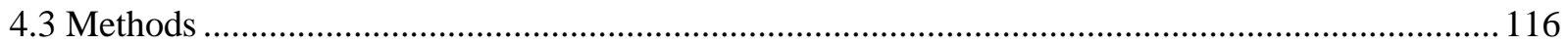

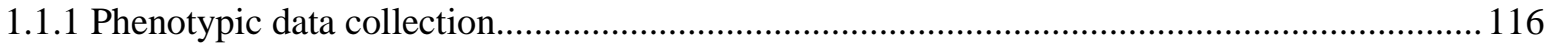

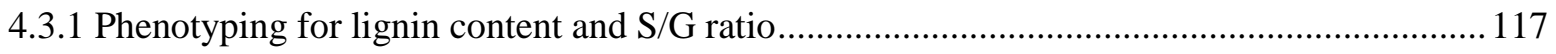

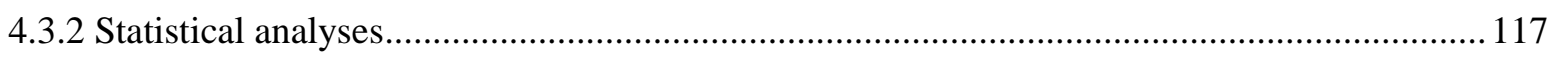

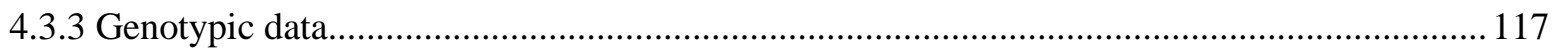

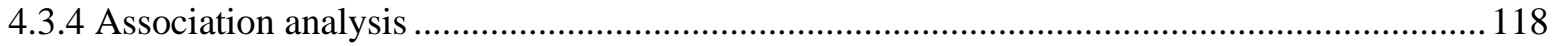

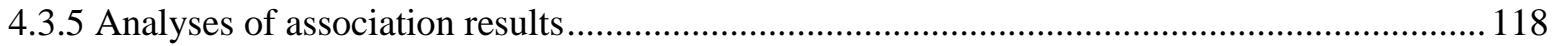

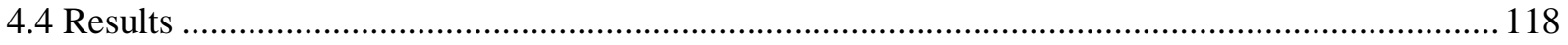

4.4.1 Heritabilities of wood anatomy, chemistry, and morphology traits ..................................... 118

4.4.2 Genetic correlation of phenotypic traits within and between the common gardens................ 119

4.4.3 Phenotypic trait correlations with climate variables ............................................................ 119

4.4.4 Genes identified from single trait and multitrait GWAS................................................... 119

4.4.5 Genes identified for wood anatomical and wood chemistry traits ....................................... 120

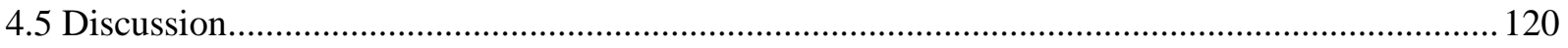

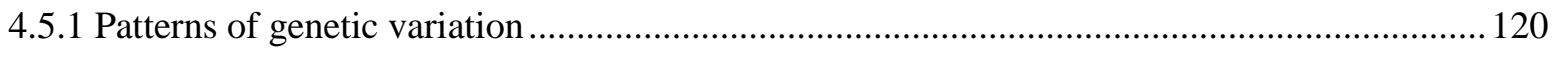

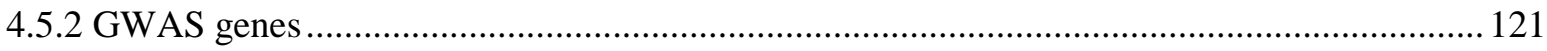

4.5.3 Gene models detected using single trait and multitrait GWAS ......................................... 122

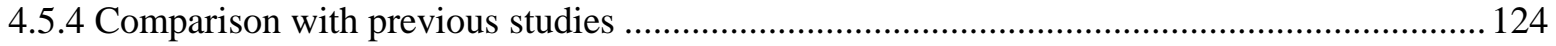

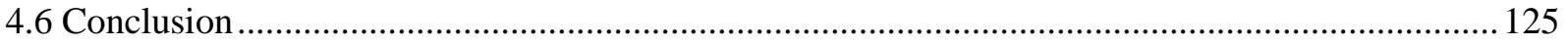

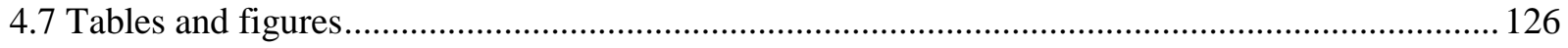

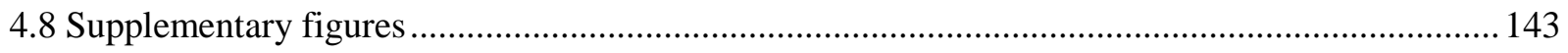

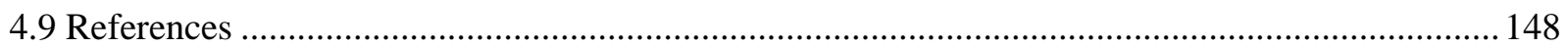

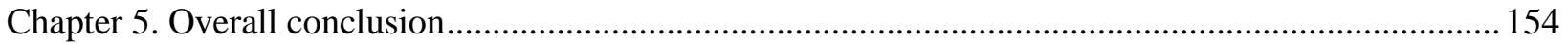




\section{List of tables}

Table 2.1 Broad-sense heritability and the number of SNP-trait associations for morphological and

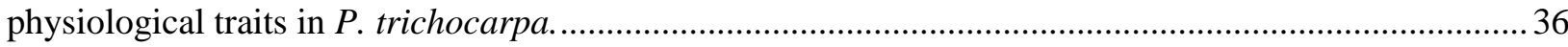

Table 2.2 List of traits for multitrait associations in P. trichocarpa ...................................................... 37

Table 2.3 Genes identified from $P$. trichocarpa single trait GWAS.................................................... 37

Table 2.4 Genes identified from P. trichocarpa multitrait GWAS. ..................................................... 38

Table 3.1 Pearson correlation (r) between 26 geo-climate variables and PCs 1 - 4 of 26 geo-climate variables of the source location of $P$. trichocarpa genotypes. Values greater than 0.13 or less than -0.13 are significant based on the Bonferroni correction criteria at 5\% significance level.

Table 3.2 PCA loadings of 26 geo-climate variables for the first 4 PCs. PC1, PC2, PC3 and PC4 explained $62.44 \%, 17.13 \%, 6.02 \%$ and $5.20 \%$ of the total variation, respectively. Dark blue and dark red colors indicate high positive and high negative values, respectively.

Table 3.3 Pearson correlation between phenotypic traits and geo-climate variables and PCs of 26 geoclimate variables. Values greater than 0.12 or less than -0.12 are significant at the $5 \%$ Bonferroni correction level of significance. Dark blue and dark red colors indicate high positive and high negative $P$ values, respectively.

Table 3.4 Genes identified from $P$. trichocarpa single and multitrait GWAS shared by climate and phenotype RDA models.

Table 4.1 Broad-sense heritability estimates $\left(H^{2}\right)$ and the number of SNP-trait associations for wood chemistry and anatomical and morphological traits in P. trichocarpa. 126

Table 4.2 List of traits used for multitrait associations and significant SNPs identified in P. trichocarpa.

Table 4.3 Pairwise correlation (r) of $P$. trichocarpa wood chemistry, anatomical and morphological traits in Clatskanie, OR. Values greater than 0.14 or less than -0.14 are significant based on the Bonferroni correction criteria at $5 \%$ significance level.

Table 4.4 Pearson correlation (r) of P. trichocarpa wood chemistry, anatomical and morpholgical traits in Clatskanie, OR with morphological and physiological traits in Corvallis, OR. Values greater than 0.14 or less than -0.14 are significant based on the Bonferroni correction criteria at 5\% significance level........ 130

Table 4.5 Pearson correlation (r) of $P$. trichocarpa wood chemistry, anatomical and morphological traits in Clatskanie, OR with morphological and physiological traits in Corvallis, OR. Values greater than 0.15 or less than -0.15 are significant based on the Bonferroni correction criteria at 5\% significance level... 131

Table 4.6 Genes identified from P. trichocarpa single trait GWAS..................................................... 132

Table 4.7 Genes identified from P. trichocarpa multitrait GWAS. 133

Supplementary Table S 2.1 SNP PC covariates used in P. trichocarpa single and multitrait GWAS analyses.

Supplementary Table S 2.2 Pearson pairwise correlation of morphological and physiological traits collected in $P$. trichocarpa association population; the numbers below diagonal represent correlation values; the numbers above diagonal represent $P$-values. Red and blue colors indicate positive and negative correlations or $P$-values, respectively. 
Supplementary Table S 2.3 PCA loadings of the traits of 13 morphological and physiological traits used in PCAbiplot (Figure 2.3) collected in P. trichocarpa association plantation in Corvallis, OR. Red and blue colors indicate positive and negative loadings, respectively. The first five PCs explain more than $83 \%$ of the variation in the traits. PC1, PC2, PC3, PC4 and PC5 explain 47\%, 14\%, 9\%, 8\% and 6\% of the total variation, respectively.

Supplementary Table S 2.4 Pearson correlation of morphological and physiological traits with latitude of origin in P. trichocarpa.

Supplementary Table S 3.1 Pearson correlation (r) of significant SNP eigenvectors from Clim and Pheno RDA models (Model 3 and 4) with selected geo-climate and first 4 PCs of 26 geo-climate variables. Values with variable color coding are $r$ values with less than or greater than 0.12 being significant based on Bonferroni correction criteria at $5 \%$ level of significance. 


\section{List of figures}

Figure 2.1 Source locations of 882 P. trichocarpa genotypes sampled in this study (colored dots). The trees were grown in a common garden in Corvallis, Oregon (black star).

Figure 2.2 Pairwise Pearson genetic correlation of selected morphological and physiological traits (traits with at least 681 genotypes) measured in the P. trichocarpa common garden in Corvallis, Oregon. The color spectrum, bright red to bright blue represents highly positive to highly negative correlations and the number represents the correlation values. Best Linear Unbiased Predictor (BLUP) adjusted values were used. $P$-values are provided in Supplementary Table S2.2. SPAD represents leaf greenness. AR, aspect ratio; SLA, specific leaf area.

Figure 2.3 PCA biplot showing the first and second principal components with individual $P$. trichocarpa genotypes (the points) colored by provenance as in Figure 2.1, and relative weightings of the explanatory variables indicated by vectors. BC, British Columbia; OR, Oregon. .42

Figure 2.4 $P$. trichocarpa SNP-trait association peak counts at 10kb intervals. (a) Single trait GWAS (b) Multitrait GWAS.

Figure 2.5 Manhattan plots comparing GEMMA univariate and multivariate GWAS in P. trichocarpa. The colors of the dots correspond to single trait or multitrait associations. $P$-values are converted to $\log 10$ ( $P$-value). SNPs above red lines passed Bonferroni correction test $\left(P<7.37 \times 10^{-9}\right)$, SNPs above blue lines are considered suggestive associations $\left(P<1 \times 10^{-7}\right)$. Only SNPs with $P<1 \times 10^{-3}$ are plotted. (a) Carbon isotope, stomatal density, and leaf area. (b) Allelic effects of SNP near Potri.009G015500. (c) Leaf area, leaf dry weight, leaf length, and leaf width. (d) Allelic effects of SNP near gene Potri.006G132500. (e) Leaf aspect ratio and specific leaf area. (f) Allelic effects of SNP near gene Potri.001G371800. SNPs depicted in Figures b, d, and $\mathrm{f}$ are circled in the corresponding Manhattan plots and the error bars in b, d, and $\mathrm{f}$ represent \pm standard error for re-scaled phenotypic values. 43

Figure 2.6 Merged network for carbon isotope, leaf area and stomatal density (CI_LA_SD) in P. trichocarpa. Networks of co-expressed genes were based on RNAseq data for 14 tissue types from the Phytozome Populus gene atlas. Networks of associated metabolites were based on GWAS for the same population that was used here (Weighill et al., 2018).....

Figure 2.7 Merged network for leaf area, leaf dry weight, leaf length, and leaf width (LA_LD_LL_LW) and leaf aspect ratio-specific leaf area (AR_SL) in P. trichocarpa. Symbols are as defined in Figure 2.6.

Figure 2.8 Effects of PdGAUT9.1 down-regulation on leaf size in P. deltoides. (a) Gene model for PtGAUT9.1 (Potri.004G111000) from Populus trichocarpa v3.0 genome. Black boxes indicate the 5' and 3' untranslated regions (UTRs); purple boxes indicate exons and lines indicate introns. The indicated RNAi targeted sequence was $123 \mathrm{bp}$. The sequences used for quantitative RT-PCR are indicated by arrows. (b) Schematic representation of PtGAUT9.1 RNAi silencing construct used to generate $P$. deltoides PdGAUT9.1-KD transgenic lines. (c) Relative transcript abundance of PdGAUT9.1 (Potri.004G111000) and PdGAUT9.2 (Potri.017G106800) as determined by quantitative RT-PCR analysis of leaf RNA from greenhouse-grown 3-month-old poplar WT, vector control (V. control.1) and PdGAUT9.1-KD lines (KH28.1, KH28.3 and KH28.12). Expression of PdGAUT9.1 in poplar WT was set to 1 and $18 S$ rRNA was used as a reference gene. Error bars are SE, $\mathrm{n}=6$. Differences were tested by oneway ANOVA $(P<0.05 ; P<0.001)$. (d) Leaf phenotype (the sixth leaf from the apex) of $P$. deltoides control (WT and VC) and PdGAUT9.1-KD line (KH28.12) from 3-month-old plants. (e) Length and (f) width of leaves from different developmental stages of three different 3-month-old GAUT9-KD transgenic lines (KH28.1, KH28.3 and KH28.12). Every other leaf of ten plants was measured starting with the $2^{\text {nd }}$ leaf from the apex. The error bars represent \pm standard error for leaf length and leaf width. 
Figure 3.1 Source locations of 869 P. trichocarpa genotypes sampled in this study (purple dots). The trees were grown in a common garden in Corvallis, Oregon (black star).

Figure 3.2 Genes detected by genotype environment association (GEA) across the methods - single trait, multitrait and PC-based. (a) $P<7.417 \times 10^{-9}$ (Bonferroni correction threshold). (b) $P<1 \times 10^{-7}$. 91

Figure 3.3 Single trait, multitrait and PC based association showing the association of geo-climate variables with the SNPs in chromosome 10 - Manhattan (left) and QQ plots (right). Numbers 1 to 19 represent chromosomes; scaffolds are the reads that did not align to any of the 19 chromosomes. (a) and (b) Summer heat:moisture index (SHM) (c) and (d) Mean annual precipitation (MAP) (e) and (f) Multitrait GWAS of six climate variables - MAP, MAT (mean annual temperature), MWMT (mean warmest month temperature), RH (relative humidity), SHM and TD [temperature difference between MWMT and MCMT (mean coldest month temperature)]. (g) and (h) PC2 of 25 geo-climate variables (includes latitude, longitude and elevation, and 22 climate variables). Red horizontal line indicates Bonferroni correction threshold $\left(P=7.417 \times 10^{-9}\right)$ and blue horizontal line indicates suggestive association threshold $\left(P=1 \times 10^{-7}\right)$. Nearest gene to the highlighted SNP/s (red circle) is Potri.010G080200, which is a very long chain beta-ketoacyl-CoA synthase with potential involvement in cuticular wax biosynthesis. .93

Figure 3.4 Triplots showing RDA on SNP eigenvectors constrained by the matrix of climate variables (a), climate and space variables (b) and climate variables with space as covariates (c). Both RDA1 and RDA2 axes were significant in all RDA models. It appears that the separation of $P$. trichocarpa populations in the RDA axis 1 in the climate only RDA model is due to the confounding effect of spatial variation. Circles indicate $869 P$. trichocarpa genotypes (color coded by populations, the rivers). Triangles indicate SNP eigenvectors with outlier eigenvectors (based on a 3 standard deviation cutoff selected from the tails of the distribution of RDA axes) in black. Blue arrows indicate the influence of climate variables on RDA axis

Figure 3.5 Square loadings of top 0.1\% SNPs from one of the 5 significant eigenvectors (EV32) detected from the climate RDA analysis (a) and one of the 17 outlier eigenvectors (EV1) detected from the phenotypic RDA analysis (b). Numbers 1 to 19 represent chromosomes; scaffolds are the reads that did not align to any of the 19 chromosomes. Gray and yellow colors represent positive and negative square loadings, respectively. Red and blue circles indicate top 100 square loadings corresponding to positive and negative loadings, respectively. Climate RDA (EV32) identified the same gene (circled red) that was identified in GEA. Phenotypic RDA (EV1) also identified a similar gene, Potri.010G80300 (circled blue) just 100 bp upstream of the Potri.01G080200.

Figure 3.6 Expression level of genes identified from single and multitrait GWAS shared by climate and phenotype RDA models in different tissues in P. trichocarpa.

Figure 3.7 Partitioning of variance components in RDA analyses. (a) SNP Clim + Geo model. 7.6\% of the total variation is explained by climate and geography (matrix of spatial variable). (b) Pheno $\sim \mathrm{SNP}+$ Clim + Geo model. $22 \%$ of the total variation is explained by SNP EVs, climate and geography. 96

Figure 3.8 Comparison of significant explanatory variables (brown) from RDA model with all 11 traits and the corresponding simple multiple linear regression models. EVs represent SNP eigenvectors, Y represents a space variable, MAT, MWMT, and RH are climate variables.

Figure 4.1 Source locations of 869 P. trichocarpa genotypes sampled in this study (purple dots). The trees were grown in a common garden in Clatskanie, Oregon, USA (black star).

Figure 4.2 Representative $P$. trichocarpa stem cross section at 100x magnification. 136

Figure 4.3 Single trait GWAS - Manhattan (left) and QQ plots (right). Numbers 1 to 19 represent chromosomes; scaffolds are the reads that did not align to any of the 19 chromosomes. (a) and (b) DBH; (c) and (d) Leaf circularity; (e) and (f) Leaf dry weight; (g) and (h) Leaf wet weight; (i) and (j) Petiole maximum diameter; (k) and (l) Abaxial stomatal density; (m) and (n) Intermediate wood vessel size. Red 
horizontal line indicates Bonferroni correction threshold $\left(P=7.417 \times 10^{-9}\right)$ and blue horizontal line indicates suggestive association threshold $\left(P=1 \times 10^{-7}\right)$.

Figure 4.4 Multitrait GWAS - Manhattan (left) and QQ plots (right). Numbers 1 to 19 represent chromosomes; scaffolds are the reads that did not align to any of the 19 chromosomes. (a) and (b) Late wood vessel area and Late wood vessel count; (c) and (d) Leaf area, leaf dry weight, leaf length and leaf wet weight. Red horizontal line indicates Bonferroni correction threshold $\left(P=7.417 \times 10^{-9}\right)$ and blue horizontal line indicates suggestive association threshold $\left(P=1 \times 10^{-7}\right)$.

Figure 4.5 Expression level of gene models identified from single trait GWAS in different tissues in $P$. trichocarpa.

Figure 4.6 Expression level of gene models identified from multitrait GWAS in different tissues in $P$. trichocarpa.

Supplementary Figure S 2.1 Manhattan plots comparing GEMMA univariate and multivariate GWAS in $P$. trichocarpa. The colors of the dots correspond to single trait or multitrait (purple) associations. $P$ values are converted to $-\log 10$ ( $P$-value). SNPs above red lines passed Bonferroni correction test $\left(P<7.37 \times 10^{-9}\right)$, SNPs above blue lines are considered suggestive associations $\left(P<1 \times 10^{-7}\right)$. Only SNPs with $P<1 \times 10^{-3}$ are plotted. (a) Stomatal density (green), carbon isotope (red), and pre-dawn leaf water potential (blue). (b) Height (green), leaf area (blue), and petiole length (red). (c) Height (green), petiole diameter (blue), and petiole length (red)

Supplementary Figure S 2.2 Manhattan plots comparing GEMMA univariate and multivariate GWAS in $P$. trichocarpa. The colors of the dots correspond to single trait or multitrait (purple) associations. $P$ values are converted to $-\log 10(P$-value $)$. SNPs above blue lines are considered suggestive associations $\left(P<1 \times 10^{-7}\right)$. Only SNPs with $P<1 \times 10^{-3}$ are plotted. (a) Leaf area (blue), stomatal density (green), and SPAD (red). (b) Leaf area (blue), petiole length (green), height (yellow), and carbon isotope (red). (c) Leaf area (blue), stomatal density (green), SPAD (yellow), and carbon isotope (red).

Supplementary Figure S 2.3 Manhattan plots comparing GEMMA univariate and multivariate GWAS in $P$. trichocarpa. The colors of the dots correspond to single trait or multitrait (purple) associations. $P$ values are converted to $-\log 10(P$-value). SNPs above blue lines are considered suggestive associations $\left(P<1 \times 10^{-7}\right)$. Only SNPs with $P<1 \times 10^{-3}$ are plotted. (a) Carbon isotope (red), pre-dawn leaf water potential (blue). (b) Leaf dry weight (red), petiole diameter (green), SPAD (blue). (c) Petiole diameter (green), petiole length (red), SLA (blue).

Supplementary Figure S 2.4 QQ-plot for single trait GWAS in P. trichocarpa for carbon isotope composition (a), leaf area (b), and stomatal density (c) and the corresponding multitrait GWAS with all 3 traits (d). For corresponding Manhattan Plots, see Figure 2.5a. .55

Supplementary Figure S 2.5 QQ-plot for single trait GWAS in P. trichocarpa for leaf area (a), leaf dry weight (b), leaf length (c) and leaf width (d) and the corresponding multitrait GWAS with all 4 leaf traits (e). See Figure 2.5c for the corresponding Manhattan plots.

Supplementary Figure S 2.6 QQ-plot for single trait GWAS in P. trichocarpa for leaf aspect ratio (a) and specific leaf area (b), and the corresponding multitrait GWAS with both traits (c). See Figure 2.5e for the corresponding Manhattan plots.

Supplementary Figure S 2.7 Pearson correlation of GAUT9 (Potri.004G111000) gene expression in (a) leaf and (b) developing xylem of $P$. trichocarpa with genotype at locus Chr04_9996091. Big boxes represent interquartile range with black horizontal bar within the box representing median expression value. The lower and upper whiskers represent 1.5 times the interquartile range, and the circles represent outliers. 
Supplementary Figure S 2.8 Allelic effects plots for single traits underlying the carbon isotope-leaf area-stomatal density multitrait association analysis in $P$. trichocarapa. Locus position is indicated above the plot. The error bars represent standard errors for re-scaled phenotypic values. (a) Locus

Chr01_43644555; (b) Locus Chr01_43644561; (c) Locus Chr02_10821727; (d) Locus Chr02_10821794.

Supplementary Figure S 2.9 Allelic effects plots for single traits underlying the LA_LD_LL_LW association analysis in P. trichocarpa. Locus position is indicated above the plot. Numbers in parentheses below the plot are the number of observations of each genotype. Error bars represent standard errors for re-scaled phenotypic values. LA, leaf area; LD, leaf dry weight; LL, leaf length; LW, leaf width. (a) Locus Chr01_14742176; (b) Locus Chr01_14769998; (c) Locus Chr02_3703017; (d) Locus Chr04_9996091; (e) Locus Chr06_10894444; (f) Locus Chr06_10894482; (g) Locus Chr08_9674908; (h) Locus Chr10_16268015; (i) Locus Chr10_16268028; (j) Locus Chr19_2437823.

Supplementary Figure S 2.10 Allelic effects plots for single traits underlying the AR_SLA association analysis in $P$. trichocarpa. Locus position is indicated above the plot. Numbers in parentheses below the plot are the number of observations of each genotype. Error bars represent standard errors for re-scaled phenotypic values. AR, aspect ratio; SLA, specific leaf area. (a) Locus Chr01_38557469; (b) Locus Chr03_17588967; (c) Locus Chr03_17588972; (d) Locus Chr08_752568.

Supplementary Figure S 3.1 Geography and climate maps for the range of $P$. trichocarpa distribution. (a) Elevation; (b) Mean annual temperature (MAT); (c) Mean coldest month temperature (MCMT); (d) Mean warmest month temperature (MWMT); (e) Summer heat:moisture index (SHM); (f) Annual heat:moisture index (AHM); (g) Mean annual relative humidity $(\mathrm{RH})$.

Supplementary Figure S 3.2 Single trait GWAS showing the association of climate variables with the SNPs in the genome - Manhattan (left) and QQ (right) plots. Numbers 1 to 19 represent chromosomes; scaffolds are the reads that did not align to any of the 19 chromosomes. (a) and (b) MAT (Mean annual temperature); (c) and (d) MWMT (Mean warmest month temperature); (e) and (f) TD (Temperature difference between MWMT and MCMT (Mean coldest month temperature); (g) and (h) RH (Mean annual relative humidity); (i) and (j) PC1 of 25 geo-climate variables (includes latitude, longitude and elevation, and 22 climate variables). Red horizontal line indicates Bonferroni correction threshold $\left(P=7.417 \times 10^{-9}\right)$ and blue horizontal line indicates suggestive association threshold $\left(P=1 \times 10^{-7}\right)$.

Supplementary Figure S 3.3 RDA triplot with the response matrix of 113 SNP eigenvectors constrained by the matrix of climate variables [(a) and (b)], climate and space variables [(c) and (d)] and climate variables conditioned on space variables [(e) and (f)]. Circles indicate 869 P. trichocarpa genotypes (color coded by population). Triangles indicate SNP eigenvectors with outlier eigenvectors (based on a 3 standard deviation cutoff selected from the tails of the distribution of RDA axes) in black. Blue arrows indicate the influence of climate variables on RDA axis.

Supplementary Figure S 3.4 RDA triplot with the response matrix of 11 phenotypic traits (large colored circles) constrained by the matrices of 113 SNP eigenvectors and 5 climate variables with the spatial matrix used as covariate. Arrows indicate variable containing SNP eigenvectors and climate with blue indicating climate variables and red indicating significant variables in the RDA model. Small gray circles indicate 869 P. trichocarpa genotypes. 103

Supplementary Figure S 3.5 Square loadings of top 0.1\% SNPs of outlier eigenvectors (EVs) detected from the climate RDA analysis (a) EV2 (b) EV3 (c) EV5 (d) EV8. Numbers 1 to 19 represent chromosomes; scaffolds are the reads that did not align to any of the 19 chromosomes. Gray and yellow colors represent positive and negative square loadings, respectively. Red and blue indicate top 100 square loadings corresponding to positive and negative loadings, respectively. 104

Supplementary Figure S 3.6 Square loadings of top 0.1\% SNPs of outlier eigenvectors (EVs) detected from the phenotypic RDA analysis (a) EV6 (b) EV18 (c) EV22 (d) EV23 (e) EV27 (f) EV31 (g) EV44 (h) 
EV46 (i) EV59 (j) EV60 (k) EV62 (1) EV72 (m) EV93 (n) EV106 (0) EV107. Numbers 1 to 19 represent chromosomes; scaffolds are the reads that did not align to any of the 19 chromosomes. Gray and yellow colors represent positive and negative square loadings, respectively. Red and blue indicate top 100 square loadings corresponding to positive and negative loadings, respectively. 106

Supplementary Figure S 4.1 Single trait GWAS - Manhattan (left) and QQ plot (right). Numbers 1 to 19 represent chromosomes; scaffolds are the reads that did not align to any of the 19 chromosomes. (a) and (b) Intermediate wood vessel count; (c) and (d) Late wood vessel size; (e) and (f) S/G ratio; (g) and (h) Leaf aspect ratio. Red horizontal line indicates Bonferroni correction threshold $\left(P=7.417 \times 10^{-9}\right)$ and blue horizontal line indicates suggestive association threshold $\left(P=1 \times 10^{-7}\right)$.

Supplementary Figure S 4.2 Multitrait GWAS - Manhattan (left) and QQ plot (right). Numbers 1 to 19 represent chromosomes; scaffolds are the reads that did not align to any of the 19 chromosomes. (a) and (b) Early wood vessel area, leaf area and stomatal density; (c) and (d) Early wood vessel size, intermediate wood vessel size, lignin content, S/G ratio and late wood vessel size; (e) and (f) Intermediate wood vessel size, lignin content and S/G ratio; (g) and (h) Lignin content, late wood vessel size and S/G ratio; (i) and (j) Lignin content and S/G ratio; (k) and (1) Lignin content, specific leaf area, stomatal density and early wood vessel area; (m) and (n) Late wood vessel area and late wood vessel count. Red horizontal line indicates Bonferroni correction threshold $\left(P=7.417 \times 10^{-9}\right)$ and blue horizontal line indicates suggestive association threshold $\left(P=1 \times 10^{-7}\right)$. 


\section{Chapter 1. General introduction}

\subsection{Introduction}

Understanding the genetic basis of a trait is of fundamental importance in biology. Various abiotic and biotic factors are known to have an effect on the genetic variation and evolution of a species (Bragg, Supple, Andrew, \& Borevitz, 2015; Lind, Menon, Bolte, Faske, \& Eckert, 2018; Richardson, Urban, Bolnick, \& Skelly, 2014; Sork et al., 2013). Forest trees like Populus that occur over a wide geographical range serve as an excellent model system for mechanistic studies of adaptation. The power of advanced sequencing technology coupled with clonally replicated common gardens has helped to accelerate the process of finding links between genotypes and phenotypes. Unraveling the genetic mechanisms underlying key structural and functional traits, thus understanding local adaptation, facilitates the development of Populus as a bioenergy crop.

Nevertheless, most morphological, anatomical and physiological traits are complex, and detection of underlying mechanisms is often not straightforward. The traditional way of detecting loci controlling adaptive traits- QTL mapping- is cumbersome and not feasible in many forest trees because of the requirement of a multigenerational pedigree that takes many years to develop. Furthermore, QTL mapping lacks precision because the detected underlying genetic region is not very precise due to lack of recombination, and it lacks generality because the QTL pedigree is not representative of the large genotypic range of populations (Ingvarsson, 2010). In this regard, association genetics has emerged as a powerful tool for assessing the genetic mechanisms of complex traits because it captures the variation present in natural populations (Neale \& Savolainen, 2004). One of the biggest advantages of association mapping is that wild natural populations can be used directly, and there is no need to generate a pedigree. Furthermore, loci and alleles underlying the traits can be precisely located and characterized at the nucleotide level, though the realized resolution depends greatly on the background linkage disequilibrium (Balding, 2006). With the vast improvement in sequencing technologies in recent years, whole genome association scans are possible at a low cost. For a tree like Populus that has a moderate genome size (about $450 \mathrm{Mb}$ ) sequencing hundreds of individuals in a population at a reasonable depth is possible (Evans et al., 2014). However, confounding factors such as population structure can cause spurious correlations, which needs to be taken into account for association studies (Slavov, Leonardi, Adams, Strauss, \& DiFazio, 2010).

Forest trees are excellent model systems for studying local adaptation. Forest trees are perennials and have long generation times, span over large geographic areas, and harbor high genetic and phenotypic variation. They often cover large range of environments and show latitudinal clines for various adaptive traits (Grattapaglia, Plomion, Kirst, \& Sederoff, 2009; Neale \& Kremer, 2011; Savolainen \& Pyhäjärvi, 
2007). Populus is a particularly good model tree genus due to its wide distribution, fast growth, moderate genome size, hybrid vigor, ease of propagation and high ecological and economic value. A wealth of genomic resources is available for the genus. Populus trichocarpa is one of the most intensively-studied species in the genus. It is a dominant riparian species that has a distribution from northern California to northern British Columbia. It is a pioneer species that plays an important role in riparian ecosystems (Cronk, 2005; Eckenwalder, Stettler, Bradshaw Jr., Heilman, \& Hinckley, 1996), and it is being developed as a biofuel feedstock (Tuskan, 1998). Genomic resources for this species include a whole genome sequence (Tuskan et al., 2006), extensive resequencing data for a large association population (Chhetri et al., 2019; Evans et al., 2014; Weighill et al., 2018), a SNP array targeting 34,000 genomewide loci (Geraldes et al., 2013), exome sequencing (Zhou, Bawa, \& Holliday, 2014) and extensive transcriptome libraries (Jansson \& Douglas, 2007). Furthermore, high levels of adaptive phenotypic variation is present in P. trichocarpa (Chhetri et al., 2019; Mckown, Guy, et al., 2014; McKown et al., 2014; Mckown, Klápště, et al., 2014). In addition, linkage disequilibrium (LD) in Populus is very low on average. Low LD coupled with high-resolution SNP markers in Populus can be a big asset for precisely locating QTL alleles in the genome.

Studies on association genetics of forest trees have shown that nucleotide polymorphisms within candidate genes account for significant variation in complex traits in natural populations (Eckert et al., 2009; González-Martínez, Wheeler, Ersoz, Nelson, \& Neale, 2007; Ingvarsson, Garcia, Luquez, Hall, \& Jansson, 2008; Rellstab, Gugerli, Eckert, Hancock, \& Holderegger, 2015; Wegrzyn et al., 2010). However, most of these association studies are either not comprehensive in terms of the traits studied and/or are based on a priori selection of targeted genomic regions that ignore a significant part of the genome. In recent years, these efforts have been expanded to the whole genome level and focused on morphological, structural and physiological traits. Numerous genes and genomic regions controlling quantitative traits have been identified in P. trichocarpa (Bdeir et al., 2019; Chhetri et al., 2019; Evans et al., 2014; McKown et al., 2014; Mckown, Klápště, et al., 2014; Muchero et al., 2015; Wegrzyn et al., 2010). Tremendous progress has been made in finding allelic variants associated with cell wall chemistry (Muchero et al., 2015; Wegrzyn et al., 2010). QTLs for lignin content, syringyl to guaiacyl (S/G) ratio, and 5- and 6-carbon sugars were detected and validated from a QTL and multiple association populations (Muchero et al., 2015). Furthermore, insights have been gained into mechanisms underlying local adaptation, including the role of structural polymorphisms and gene duplication in adaptive trait evolution (Evans et al., 2014).

Forest tree populations typically show local adaptation, in which local genotypes perform better than genotypes from other locations (Jump \& Penuelas, 2005; Savolainen, Lascoux, \& Merilä, 2013). This poses challenges in the face of rapid climate change because locally advantageous genotypes could 
become maladapted. Local populations could respond to climate change by altering genetic composition through gene flow and recombination, which in turn will aid in modifying the structural and functional traits for local adaptation. However, it is unclear if gene flow will be sufficient to respond to the rapid rate of climate change (Aitken, Yeaman, Holliday, Wang, \& Curtis-McLane, 2008; Slavov et al., 2004; Xie, Carlson, \& Ying, 2012). A combination of comprehensive genomic data and field experiments is required for understanding the genetics of local adaptation (Savolainen, Lascoux \& Merila, 2013). There has been some progress in understanding the genetic mechanisms underlying ecologically and economically important traits (Bdeir et al., 2019; Cumbie et al., 2011; Du et al., 2019; Evans et al., 2014; GonzálezMartínez, Huber, Ersoz, Davis, \& Neale, 2008; González-Martínez et al., 2007; Ingvarsson, 2010; McKown et al., 2014; Mckown, Klápště, et al., 2014; Muchero et al., 2018, 2015; Neale \& Savolainen, 2004; Parchman et al., 2012; Porth et al., 2013; Wang et al., 2018; Wegrzyn et al., 2010; Wei et al., 2014; Zhang et al., 2018), but comprehensive study of morphological, anatomical and physiological traits is still rare in forest trees. We will use an association genetics approach to study key physiological and adaptive traits in P. trichocarpa. The complementary aspect of this study is to understand mechanisms underlying local adaptation in Populus by employing various differentiation (genome scan), genotype-environment association (GEA) and multivariate methods such as redundancy analysis (RDA) (Capblancq, Luu, Blum, \& Bazin, 2018; Duforet-Frebourg, Luu, Laval, Bazin, \& Blum, 2016; Forester, Lasky, Wagner, \& Urban, 2018; Frichot, Schoville, Bouchard, \& François, 2013; Lind et al., 2018; Rellstab et al., 2015).

Better understanding of physical traits (e.g. pulp yield), chemical traits (e.g. cellulose and lignin content), morphological traits [e.g. specific leaf area (SLA) and petiole length], physiological traits (e.g. leaf water potential) as well as anatomical traits (e.g. cell wall thickness and size and distribution of xylem vessels) including their interactions is also of utmost importance to optimize biomass as a major source of bioenergy. Thus far there have been no published studies of the genetic and molecular mechanisms underlying important wood anatomical traits such as xylem vessel size and density. This study aims to provide information for detecting genes responsible for these key anatomical, morphological and physiological traits via an association genetics approach, which may in turn be instrumental in providing a framework for developing Populus as a sustainable bioenergy crop. A complementary aspect of this study is to assess the genetic mechanism underlying local adaptation in Populus.

\subsection{Leaf morphology and function}

A wide range of genetic variation has been reported for leaf morphology in P. trichocarpa (Gilchrist et al., 2006). Plasticity of leaf traits allows plants to adjust to a wide range of environmental conditions. Trees subjected to drought typically have decreased leaf area, leaf number, and specific leaf area along with decreased photosynthetic rate, transpiration, stomatal conductance $\left(\mathrm{g}_{\mathrm{s}}\right)$, intercellular $\mathrm{CO}_{2}$ 
concentration $\left(\mathrm{c}_{\mathrm{i}}\right)$, maximum efficiency of PSII $\left(\mathrm{F}_{\mathrm{v}} / \mathrm{F}_{\mathrm{m}}\right)$ and maximum effective quantum yield of PSII (Yield) as reported for some Populus species (Xiao, Xu, \& Yang, 2008; Xu et al., 2008). P. trichocarpa trees in higher latitudes have greater leaf longevity that allows them to compensate for reduced growth and photosynthetic rate (Gornall \& Guy, 2007). Trees in high latitudes are also expected to have thicker leaves. Furthermore, the alkene composition of the cuticle has been found to be associated with productivity and water use efficiency in natural P. trichocarpa populations (Gonzales-Vigil, Hefer, von Loessl, La Mantia, \& Mansfield, 2017). Significant variation in leaf characters among populations has been found in the populations of another important species of the genus, P. deltoides in Ontario. Vein numbers in the leaf were shown to have significant correlation with latitude and longitude (Rajora, Zsuffa, \& Dancik, 1991). Variation in leaf morphology has great implications for plants in regulating photosynthetic rate, growth and adaptation to a changing climate.

\subsection{Stomata}

Variation in stomatal size and density in the abaxial and adaxial leaf surface affects stomatal conductance which in turn affects net $\mathrm{CO}_{2}$ assimilation (A) in plants (Pearce, Millard, Bray, \& Rood, 2006). Studies have shown that the stomatal density increases and the size decreases in P. trichocarpa and other Populus species with an increase in latitude and in xeric conditions (Sparks \& Black, 1999). Interestingly, the adaxial leaf stomata in P. trichocarpa, although scant in distribution, have shown strong correlation with latitude, thus facilitating higher stomatal conductance and net $\mathrm{CO}_{2}$ assimilation in the regions with short growing seasons (Gornall \& Guy, 2007; McKown et al., 2014). Studies on $P$. trichocarpa and other Populus species have shown that more variation in total stomatal densities (abaxial plus adaxial) exists within species than between species. However, abaxial and adaxial stomatal densities and adaxial to abaxial density ratio differed across species suggesting adaptation to different environmental niches (Pearce et al., 2006). Several studies have shown that the regulation of stomatal density and stomatal aperture play a great role in acclimation and adaptation to abiotic stress (Ceulemans, Impens, \& Steenackers, 1988; Ceulemans, Praet, \& Jiang, 1995). This information can be useful for selecting Populus clones suitable for various climatic regions.

\subsection{Carbon isotope composition}

Carbon isotope composition provides evidence for water use efficiency in plants. There are two ways by which the water use efficiency in plants can be measured. The ratio $\left(\mathrm{A} / \mathrm{g}_{\mathrm{s}}\right)$ of $\mathrm{CO}_{2}$ assimilation (A) to stomatal conductance $\left(\mathrm{g}_{\mathrm{s}}\right)$ provides a measure of instantaneous intrinsic water use efficiency (WUEi) whereas the stable carbon isotope composition $\left(\delta^{13} \mathrm{C}\right)$ provides a long-term measure of water use efficiency (WUE) in plants and is less prone to short-term environmental fluctuations.

Plants in general have affinity towards the lighter carbon isotope, ${ }^{12} \mathrm{C}$ over ${ }^{13} \mathrm{C}$, which is commonly referred to as carbon isotope discrimination. When intercellular $\mathrm{CO}_{2}$ concentration $\left(\mathrm{c}_{\mathrm{i}}\right)$ is low, 
discrimination decreases and is reflected as a less negative value. The negative value of discrimination is not just due to the intercellular $\mathrm{CO}_{2}$ concentration inside the leaf. About $27 \mathrm{ppm}$ of the fractionation is due to the affinity of Rubisco with ${ }^{12} \mathrm{C}$, and $4 \mathrm{ppm}$ is due to the diffusion properties of the leaf epidermis. The net discrimination, a long-term measure of WUE is calculated as follows (Farquhar, Ehleringer, \& Hubick, 1989):

$\Delta=\delta^{13} C_{\text {air }}-\frac{\delta^{13} C_{\text {plant }}}{1,000+\delta^{13} C_{\text {plant }}} \times 1,000$,

where $\Delta$ is the net carbon isotope discrimination, and $\delta^{13} C_{\text {air }}\left(\mathrm{c}_{\mathrm{a}}\right)$ and $\delta^{13} C_{\text {plant }}\left(\mathrm{c}_{\mathrm{i}}\right)$ are carbon isotope composition of the source air and the plant, respectively.

More negative values of $\delta^{13} \mathrm{C}$ are often associated with lower WUE and higher stomatal conductance (Gornall \& Guy, 2007; McCarroll \& Loader, 2004). If $\mathrm{c}_{\mathrm{i}}$ in the leaf is higher relative to $\mathrm{c}_{\mathrm{a}}$, then it suggests that the stomatal conductance is occurring at a much higher rate than photosynthesis, so that the discrimination against ${ }^{13} \mathrm{C}$ is greater, giving low $\delta^{13} \mathrm{C}$ values. $P$. trichocarpa clones are known to have strong genetic variation for $\delta^{13} \mathrm{C}$ (Gornall \& Guy, 2007). Understanding how plants respond to limited water availability measured by net discrimination has important ecological implications.

\subsection{Pre-dawn leaf water potential}

Predawn leaf water potential $(\Psi)$ is the measure of plant water status which is expected to be in equilibrium with the soil water potential (Fu, Chen, \& Li, 2010; Richter, 1997) and represents the energy level of the solution drawn from the soil. The method has been used as a proxy for determining the irrigation timing in crop plants, which is an integrated measure of soil, plant and atmosphere on plant water use. Water potential is a negative number measured in reference to pure water, which has zero water potential. It is measured in bars, where 10 bars is equivalent to $1 \mathrm{MPa}$. A similar measurement, called leaf hydraulic conductance $\left(\mathrm{K}_{\text {leaf }}\right)$, is a measure of the ratio of the flow rate of water to the potential gradient across the leaf and gives an idea of how a plant is responding to the internal and external environment. It is a better measurement than the leaf water potential measurement, but is time consuming (Sack \& Holbrook, 2006; Sack \& Scoffoni, 2012). Factors such as stomatal conductance and transpiration that affect leaf water potential can have correlations with hydraulic conductance (Sperry, 2000). Drought tolerant species have been shown to have a linear decline in leaf water potential (more negative $\Psi$ value) at $80 \%$ loss of $\mathrm{K}_{\text {leaf }}$ (Scoffoni, McKown, Rawls, \& Sack, 2012).

Low water potential in plants can cause stomatal closure and affect photosynthesis. Low water potential in xylem can lead to cavitation affecting the movement of water to the leaves (Sperry, 2000). A study on stem and leaf water potential in $P$. euphratica and $P$. russkii showed variation in moisture retaining capacity between the species (Fu et al., 2010). Another study on P. tremula showed a correlation between stomatal conductance and stomatal sensitivity to an increase in leaf water potential (Aasamaa, 
Sober, \& Rahi, 2001). Under stressed conditions (low water potential) P. tremula showed an increased concentration of abscisic acid (ABA) leading to a decrease in stomatal conductance. At water potential lower than -0.7 MPa, P. balsamifera showed vulnerability to xylem cavitation (Hacke \& Sauter, 1995). $P$. trichocarpa clones have the capability to respond to decreasing leaf water potential by rapidly closing stomata. $P$. trichocarpa populations from high potential evaporation environments have high resistance to drought-induced xylem cavitation (Sparks \& Black, 1999).

\subsection{Wood anatomy}

Wood is primarily comprised of cellulose and lignin, two of the most abundant polymers on earth (Groover, Nieminen, Helariutta, \& Mansfield, 2010). The internal anatomy of wood is relatively simple, yet the mechanisms regulating the genetic and molecular basis of wood formation are highly complex. The formation of wood is due to the primary growth that occurs in the early stages of development and the secondary growth that occurs after the primary growth ceases to function (Evert, 2006). Cell walls are a major component of wood and provide rigidity to the cell. The major polymers found in the plant cell wall are cellulose, hemicellulose, pectin and lignin. While lignin is the major component providing mechanical support to the cell and the plant body, it is a major challenge for the enzymatic conversion of polysaccharides to ethanol because of its interference with the enzymatic process (DOE. U.S., 2010).

Anatomically wood is made up of three different xylem tissues in angiosperms - vessels, rays and fibers. Vessels are the main conducting tissues that help in longitudinal transport of water. Rays are physiologically active parenchymatous cells that serve for carbon storage as well as the transport of resources radially between xylem and phloem. Fibers provide the strength. Wood anatomical traits are some of the key functional traits that provide stability and architecture to the tree, provide defense against insects and pathogens, and regulate hydraulic conductivity. Trees with larger vessels generally have low wood density, higher cellulose content and are associated with fast growing ability. In contrast, trees with smaller vessels have high wood density and are associated with slow growth and higher lignin content, but have high survival capability because of hydraulic safety and resistance against damage from herbivores and pathogens. Both size and the number of vessels affect hydraulic conductance in trees (Sperry, Hacke, \& Pittermann, 2006). According to the Hagen-Poiseuille law hydraulic conductance of a cylindrical vessel increases with the forth power of the vessel radius (Schuldt, Leuschner, Brock, \& Horna, 2013). Trees with larger vessels have higher hydraulic conductance, higher stomatal conductance and more photosynthetic carbon gain (Santiago et al., 2004), but are prone to vessel implosion and cavitation (Hacke, Sperry, Pockman, Davis, \& McCulloh, 2001; Hacke, Sperry, Wheeler, \& Castro, 2006).

The allocation of resources to different tissue types in the wood depends on abiotic and biotic selection pressures. This would mean a change in the length and density of the vessels, number of ray 
cells or the density of fibers that can potentially affect the structural and chemical composition of the wood, ultimately affecting wood recalcitrance and plant performance (Zanne \& Falster, 2010).

Manipulating the allocation of resources in poplar wood is important for developing this species as a reliable and sustainable lignocellulosic feedstock for biofuel production.

\subsection{Local adaptation in forest trees}

Forest trees span over large geographic areas, harbor high genetic and phenotypic variation, and often show latitudinal clines for various adaptive traits (Grattapaglia et al., 2009; Neale \& Kremer, 2011; Savolainen et al., 2013; Savolainen \& Pyhäjärvi, 2007). Local adaptation is the tendency of plant populations showing better fitness in the native environment compared to the foreign habit. Classical way of testing for local adaptation is by reciprocal experiments where the plants from the two native environments are grown in each other's environment and evaluate the plant fitness. However, experiments like this are time consuming and not feasible, especially for trees. Therefore, recent works in local adaptation in forest trees are focused more on the molecular mechanism underlying local adaptation.

Various methods including differentiation and genotype-environment association (GEA) are widely popular for identifying loci conferring local adaption in forest trees (Rellstab et al., 2015). Differentiation outlier methods (genome scans) allow for detecting loci with strong allele frequency differences among populations without needing environmental or phenotypic data (Duforet-Frebourg et al., 2016; Evans et al., 2014; Günther \& Coop, 2013). In contrast GEA methods identify loci based on the associations between genetic and environmental data that are potential drivers of selection (Frichot et al., 2013; Joost et al., 2007; Stucki et al., 2017; Yoder et al., 2014). However, these methods can suffer from high false-positive (De Mita et al., 2013; Meirmans, 2012) and are not powerful enough for detecting recent and weak selection loci (Lind et al., 2018). To overcome these problems, recent studies have used multivariate methods called Redundancy Analysis (RDA) that allow for simultaneously testing the association of loci with the predictor variables (Forester et al., 2018; Legendre \& Legendre, 2012). Furthermore, adaptive traits are largely under polygenic control in natural populations (Hollinger, Pennings, \& Hermisson, 2019; Pritchard \& Di Rienzo, 2010) meaning that selection on adaptive traits might affect hundreds or thousands of loci with a minimum change in allele frequency at a given locus (Le Corre \& Kremer, 2012). It is very possible that adaptation is due to covariance among key loci rather than strong differentiation in individual loci, which increase the possibility of small-effect loci remaining undetected from their neutral genetic background (Berg \& Coop, 2014; De Villemereuil, Gaggiotti, Mouterde, \& Till-Bottraud, 2016; Le Corre \& Kremer, 2012; Lind et al., 2018; Rajora, Eckert, \& Zinck, 2016). Therefore, local adaptation methods like differentiation (genome scans) and GEA should be complemented with the use of multivariate approaches like RDA to unravel the complexity underlying adaptive genetic polymorphisms (Capblancq et al., 2018; Forester et al., 2018). 
With ever growing genomic data and the improvement in various genome scan and other differentiation and GEA methods, determining the molecular signal conferring local adaptation has become increasingly feasible. Several genes controlling cold-hardiness in conifers and genes controlling bud phenology in Populus have been identified using association genetics, differentiation, GEA and RDA methods (Capblancq et al., 2018; Eckert et al., 2010; Evans et al., 2014; Fahrenkrog et al., 2017; Vangestel, Eckert, Wegrzyn, St. Clair, \& Neale, 2018; Wang et al., 2018). However, whenever possible, an integrated approach that takes into account genomic, environmental and phenotypic data at an appropriate spatial scale should be used for better understanding the mechanism of local adaptation in the face of climate change. A few studies have highlighted the importance of this approach (Eckert et al., 2015; Talbot et al., 2017; Vangestel et al., 2018). Common gardens in general provide a powerful approach for unraveling the genetic basis of quantitative traits in a controlled environment, for testing the interactive effects of environment and genotype on phenotypes, and comparing phenotypic differentiation with neutral population genetic variation. With the increasing availability of genome sequencing data coupled with common garden phenotypic data (Chhetri et al., 2019) and environmental data from the source location of phenotypes, understanding the molecular mechanisms underlying local adaptation in the face of climate change has become more feasible in Populus.

\subsection{Main objectives}

1) Understand the genetic mechanisms underlying variation in morphological and physiological traits in P. trichocarpa.

2) Understand the factors affecting local adaptation in P. trichocarpa including the relative contribution of climate and geography variables in population structure.

3) Identify candidate genes that can be targeted to enhance productivity and reduce recalcitrance of woody bioenergy feedstocks. Is there an effect of environment in GWAS for leaf morphological traits (compare GWAS for the same traits from two different plantations)?

\subsection{Expected significance}

Lignocellulosic feedstocks, especially for fast growing trees like Populus, are considered to be more reliable and sustainable than starch based feedstocks not only for biofuel production, but also for ecosystem management and carbon balance (DeCicco, 2013; Du et al., 2018; Rubin, 2008; Simmons, Loqué, \& Ralph, 2010; Weng, Li, Bonawitz, \& Chapple, 2008; Yuan, Tiller, Al-Ahmad, Stewart, \& Stewart, 2008). Modification for biomass recalcitrance, increased biomass production, and efficient conversion of biomass into ethanol are the main challenges that need to be overcome before Populus trees

can be commercialized for biofuel production. In recent years much progress has been made in unraveling the underlying genetic mechanisms of complex traits in Populus and other forest trees. However, most prior studies are based on candidate gene or other approaches that ignore a significant part of the genome 
that might potentially be affecting the trait. Furthermore, these studies are limited either by the number of traits being studied or by the sample size (limited power). We have started to learn the underlying genetic mechanism that controls the chemical composition in the cell wall in Populus, but we do not know anything about the underlying genetic mechanisms regulating the most important anatomical and architectural traits such as vessel and fiber density, ray cell numbers, and cell wall thickness that affect the hydraulic conductivity and carbon storage in trees. Moreover, understanding local adaptation and how these functional and structural traits interact with the environment is very important for selecting the high performing trees for various environmental conditions.

This study is an attempt to provide a comprehensive survey of genetic mechanisms underlying morphological, anatomical and physiological traits that affect overall plant productivity. Unraveling the genetic architecture of complex traits relevant to whole plant productivity including anatomical, morphological and physiological traits would enhance the understanding of the biology of this species. We arguably have the largest forest tree population for genome-wide association studies. Whole genome resequencing data are available for more than 1000 genotypes. A complementary aspect of this study is to assess the local adaptation in P. trichocarpa. Understanding the genetic mechanisms underlying local adaptation using genotype-environment association (GEA) and multivariate approaches such as Redundancy Analysis (RDA) would provide a better understanding of polygenic adaptation in Populus. Furthermore, understanding the genetic and environmental components driving local adaptation is key for accelerated domestication and ultimately developing $P$. trichocarpa as a bioenergy crop.

The research is expected to identify genes and gene families related to vessel size and density, and other important plant productivity and biofuel related traits. Functional analysis of the detected polymorphisms and genes will allow better understanding of the phenotype. The knowledge thus gained can be transformed to optimize $P$. trichocarpa as a bioenergy feedstock for biomass production and efficient sugar release from lignocellulosic biomass. This knowledge can be transferred to other commercial trees such as Eucalyptus and bioenergy crops such as switchgrass and sorghum.

The present study will complement other association studies of complex traits such as bud phenology, bud flush, tree diameter and height in the same population. Extensive data collected for some of the traits such as SLA and stomatal density in more than one common garden will validate the association of a particular trait with the genomic region. This may also provide an avenue for research ultimately leading to developing Populus as a sustainable bioenergy crop. Transgenic plants with reduced lignin content, increased cellulose content, increased water use efficiency and increased biomass are possible by overexpressing or knocking out genes responsible for quantitative variation in these traits. Furthermore, understanding the molecular mechanism underlying local adaption would be important for better understanding the biology and sustainable use and management of this species. Finally, the data 
generated from this research can be incorporated into a marker-aided breeding program to accelerate the domestication of these crops for biofuels and other end uses. 


\subsection{References}

Aasamaa, K., Sober, A., \& Rahi, M. (2001). Leaf anatomical characteristics associated with shoot hydraulic conductance, stomatal conductance and stomatal sensitivity to changes of leaf water status in temperate deciduous trees. Australian Journal of Plant Physiology, 28(8), 765-774.

Aitken, S. N., Yeaman, S., Holliday, J. A., Wang, T., \& Curtis-McLane, S. (2008). Adaptation, migration or extirpation: climate change outcomes for tree populations. Evolutionary Applications, 1(1), 95111.

Balding, D. (2006). A tutorial on statistical methods for population association studies. Nat Rev Genet, 7(10), 781-791. doi: 10.1038/nrg1916

Bdeir, R., Muchero, W., Yordanov, Y., Tuskan, G. A., Busov, V., \& Gailing, O. (2019). Genome-wide association studies of bark texture in Populus trichocarpa. Tree Genetics and Genomes, 15(1). doi: $10.1007 / \mathrm{s} 11295-019-1320-2$

Berg, J. J., \& Coop, G. (2014). A Population Genetic Signal of Polygenic Adaptation. PLoS Genetics, 10(8). doi: 10.1371/journal.pgen.1004412

Bragg, J. G., Supple, M. A., Andrew, R. L., \& Borevitz, J. O. (2015). Genomic variation across landscapes: insights and applications. New Phytologist, 207, 953-967. doi: 10.1111/nph.13410

Capblancq, T., Luu, K., Blum, M. G. B., \& Bazin, E. (2018). Evaluation of redundancy analysis to identify signatures of local adaptation. Molecular Ecology Resources, 18(6), 1223-1233. doi: 10.1111/1755-0998.12906

Ceulemans, R., Impens, I., \& Steenackers, V. (1988). Genetic variation in aspects of leaf growth of Populus clones, using the leaf plastochron index. Canadian Journal of Forest Research, 18(8), 1069-1077.

Ceulemans, R., Praet, L., \& Jiang, X. N. (1995). Effects of CO2 enrichment, leaf position and clone on stomatal index and epidermal cell density in poplar (Populus). New Phytologist, 131(1), 99-107. doi: 10.1111/j.1469-8137.1995.tb03059.x

Chhetri, H. B., Macaya-sanz, D., Kainer, D., Biswal, A. K., Evans, L. M., Chen, J., ... Difazio, S. P. (2019). Multitrait genome-wide association analysis of Populus trichocarpa identifies key polymorphisms controlling morphological and physiological traits. New Phytologist, 223, 293-309. doi: 10.1111/nph.15777

Cronk, Q. C. B. (2005). Plant eco-devo: the potential of poplar as a model organism. New Phytologist, 166(1), 39-48.

Cumbie, W. P., Eckert, A., Wegrzyn, J., Whetten, R., Neale, D., \& Goldfarb, B. (2011). Association genetics of carbon isotope discrimination, height and foliar nitrogen in a natural population of Pinus taeda L. Heredity, 107(2), 105-114. doi: 10.1038/hdy.2010.168 
De Mita, S., Thuillet, A. C., Gay, L., Ahmadi, N., Manel, S., Ronfort, J., \& Vigouroux, Y. (2013).

Detecting selection along environmental gradients: Analysis of eight methods and their effectiveness for outbreeding and selfing populations. Molecular Ecology, 22(5), 1383-1399. doi:

$10.1111 / \mathrm{mec} .12182$

De Villemereuil, P., Gaggiotti, O. E., Mouterde, M., \& Till-Bottraud, I. (2016). Common garden experiments in the genomic era: New perspectives and opportunities. Heredity, 116(3), 249-254. doi: 10.1038/hdy.2015.93

DeCicco, J. M. (2013). Biofuel's carbon balance: doubts, certainties and implications. Climatic Change, 121(4), 801-814. doi: 10.1007/s10584-013-0927-9

DOE. U.S. (2010). DOE Systems Biology Knowledgebase Implementation Plan. Retrieved from http://www.genomicscience.energy.gov/compbio/

Du, Q., Lu, W., Quan, M., Xiao, L., Song, F., Li, P., ... Zhang, D. (2018). Genome-Wide Association Studies to Improve Wood Properties: Challenges and Prospects. Frontiers in Plant Science, 9(December), 1-10. doi: 10.3389/fpls.2018.01912

Du, Q., Yang, X., Xie, J., Quan, M., Xiao, L., Lu, W., ... Zhang, D. (2019). Time-specific and pleiotropic quantitative trait loci coordinately modulate stem growth in Populus. Plant Biotechnology Journal, 17(3), 608-624. doi: 10.1111/pbi.13002

Duforet-Frebourg, N., Luu, K., Laval, G., Bazin, E., \& Blum, M. G. B. (2016). Detecting genomic signatures of natural selection with principal component analysis: Application to the 1000 genomes data. Molecular Biology and Evolution, 33(4), 1082-1093. doi: 10.1093/molbev/msv334

Eckenwalder, J. E., Stettler, R. F., Bradshaw Jr., H. D., Heilman, P. E., \& Hinckley, T. M. (1996). Systematics and evolution of Populus . In Biology of Populus and its implications for management and conservation (pp. 7-32). Ottawa, Canada: NRC Research Press.

Eckert, A. J., Bower, A. D., Wegrzyn, J. L., Pande, B., Jermstad, K. D., Krutovsky, K. V., ... Neale, D. B. (2009). Association genetics of coastal Douglas fir (Pseudotsuga menziesii var. menziesii, Pinaceae). I. Cold-hardiness related traits. Genetics, 182(4), 1289-1302. doi: 10.1534/genetics.109.102350

Eckert, A. J., Maloney, P. E., Vogler, D. R., Jensen, C. E., Mix, A. D., \& Neale, D. B. (2015). Local adaptation at fine spatial scales: an example from sugar pine (Pinus lambertiana, Pinaceae). Tree Genetics and Genomes, 11(3). doi: 10.1007/s11295-015-0863-0

Eckert, A. J., Van Heerwaarden, J., Wegrzyn, J. L., Nelson, C. D., Ross-Ibarra, J., González-Martínez, S. C., \& Neale, D. B. (2010). Patterns of population structure and environmental associations to aridity across the range of loblolly pine (Pinus taeda L., Pinaceae). Genetics, 185(3), 969-982. doi: 10.1534/genetics.110.115543

Evans, L. M., Slavov, G. T., Rodgers-Melnick, E., Martin, J., Ranjan, P., Muchero, W., ... DiFazio, S. P. 
(2014). Population genomics of Populus trichocarpa identifies signatures of selection and adaptive trait associations. Nature Genetics, 46(10), 1089-1096. doi: 10.1038/ng.3075

Evert, R. F. (2006). Esau’s Plant Anatomy. Hoboken, New Jersey: John Wiley \& Sons, Inc.

Fahrenkrog, A. M., Neves, L. G., Resende, M. F. R., Dervinis, C., Davenport, R., Barbazuk, W. B., \& Kirst, M. (2017). Population genomics of the eastern cottonwood (Populus deltoides). Ecology and Evolution, 7(22), 9426-9440. doi: 10.1002/ece3.3466

Farquhar, G. D., Ehleringer, J. R., \& Hubick, K. T. (1989). Carbon isotope discrimination and photosynthesis. Ann Rev Plant Physiol Plant Mol Biol, 40, 503-537.

Forester, B. R., Lasky, J. R., Wagner, H. H., \& Urban, D. L. (2018). Comparing methods for detecting multilocus adaptation with multivariate genotype-environment associations. Molecular Ecology, 27(9), 2215-2233. doi: 10.1111/mec.14584

Frichot, E., Schoville, S. D., Bouchard, G., \& François, O. (2013). Testing for associations between loci and environmental gradients using latent factor mixed models. Molecular Biology and Evolution, 30(7), 1687-1699. doi: 10.1093/molbev/mst063

Fu, A., Chen, Y. N., \& Li, W. H. (2010). Analysis on the change of water potential of Populus euphratica Oliv. and P. Russkii Jabl under different irrigation volumes in temperate desert zone. Chinese Science Bulletin, 55(10), 965-972. doi: 10.1007/s11434-009-0459-х

Geraldes, A., Difazio, S. P., Slavov, G. T., Ranjan, P., Muchero, W., Hannemann, J., ... Tuskan, G. A. (2013). A 34K SNP genotyping array for Populus trichocarpa: Design, application to the study of natural populations and transferability to other Populus species. Molecular Ecology Resources, 13(2), 306-323. doi: 10.1111/1755-0998.12056

Gilchrist, E. J., Haughn, G. W., Ying, C. C., Otto, S. P., Zhuang, J., Cheung, D., .. Cronk, Q. C. B. (2006). Use of Ecotilling as an efficient SNP discovery tool to survey genetic variation in wild populations of Populus trichocarpa. Molecular Ecology, 15(5), 1367-1378. doi: 10.1111/j.1365294X.2006.02885.x

Gonzales-Vigil, E., Hefer, C. A., von Loessl, M. E., La Mantia, J., \& Mansfield, S. D. (2017). Exploiting Natural Variation to Uncover an Alkene Biosynthetic Enzyme in Poplar. The Plant Cell, 29(8), 2000-2015. doi: 10.1105/tpc.17.00338

González-Martínez, S. C., Huber, D., Ersoz, E., Davis, J. M., \& Neale, D. B. (2008). Association genetics in Pinus taeda L. II. Carbon isotope discrimination. Heredity, 101(1), 19-26. doi: 10.1038/hdy.2008.21

González-Martínez, S. C., Wheeler, N. C., Ersoz, E., Nelson, C. D., \& Neale, D. B. (2007). Association genetics in Pinus taeda L. I. wood property traits. Genetics, 175(1), 399-409. doi:

10.1534/genetics.106.061127 
Gornall, J. L., \& Guy, R. D. (2007). Geographic variation in ecophysiological traits of black cottonwood (Populus trichocarpa). Canadian Journal of Botany, 85(12), 1202-1213. doi: 10.1139/B07-079

Grattapaglia, D., Plomion, C., Kirst, M., \& Sederoff, R. R. (2009). Genomics of growth traits in forest trees. Current Opinion in Plant Biology, 12(2), 148-156. doi: 10.1016/j.pbi.2008.12.008

Groover, A. T., Nieminen, K., Helariutta, Y., \& Mansfield, S. D. (2010). Genetics and Genomics of Populus. In S. Jansson, R. Bhalerao, \& A. T. Groover (Eds.), Genetics and Genomics of Populus (Vol. 8, pp. 201-224). doi: 10.1007/978-1-4419-1541-2_10

Günther, T., \& Coop, G. (2013). Robust identification of local adaptation from allele frequencies. Genetics, 195(1), 205-220. doi: 10.1534/genetics.113.152462

Hacke, U. G., \& Sauter, J. J. (1995). Vulnerability of xylem to embolism in relation to leaf water potential and stomatal conductance in Fagus sylvatica f . purpurea and Populus balsamifera. Journal of Experimental Botany, 46(290), 1177-1183.

Hacke, U. G., Sperry, J. S., Pockman, W. T., Davis, S. D., \& McCulloh, K. A. (2001). Trends in wood density and structure are linked to prevention of xylem implosion by negative pressure. Oecologia, 126(4), 457-461. doi: 10.1007/s004420100628

Hacke, U. G., Sperry, J. S., Wheeler, J. K., \& Castro, L. (2006). Scaling of angiosperm xylem structure with safety and efficiency. Tree Physiology, 26(6), 689-701. doi: 10.1093/treephys/26.6.689

Hollinger, I., Pennings, P. S., \& Hermisson, J. (2019). Polygenic adaptation : From sweeps to subtle frequency shifts 2 Author summary. PLoS Genetics, 15(3), 1-26. doi: https://doi.org/10.5061/dryad.7n6vg10

Ingvarsson, P. K. (2010). Nucleotide Polymorphism, Linkage Disequilibrium and Complex Trait Dissection in Populus. In S. Jansson, R. P. Bhalerao, \& A. T. Groover (Eds.), Genetics and Genomics of Populus (Vol. 8, pp. 91-111). doi: 10.1007/978-1-4419-1541-2_5

Ingvarsson, P. K., Garcia, M. V, Luquez, V., Hall, D., \& Jansson, S. (2008). Nucleotide polymoirphism and phenotypic associations within and around the phytochrome B2 locus in European aspen (Populus tremula, Salicaceae). Genetics, 178(4), 2217-2226.

Jansson, S., \& Douglas, C. J. (2007). Populus: A model system for plant biology. Annual Review of Plant Biology, 58, 435-458.

Joost, S., Bonin, A., Bruford, M. W., Després, L., Conord, C., Erhardt, G., \& Taberlet, P. (2007). A spatial analysis method (SAM) to detect candidate loci for selection: Towards a landscape genomics approach to adaptation. Molecular Ecology, 16(18), 3955-3969. doi: 10.1111/j.1365294X.2007.03442.x

Jump, A. S., \& Penuelas, J. (2005). Running to stand still: adaptation and the response of plants to rapid climate change. Ecology Letters, 8(9), 1010-1020. 
Le Corre, V., \& Kremer, A. (2012). The genetic differentiation at quantitative trait loci under local adaptation. Molecular Ecology, 21(7), 1548-1566. doi: 10.1111/j.1365-294X.2012.05479.x

Legendre, P., \& Legendre, L. (2012). Numerical Ecology. Elsevier.

Lind, B. M., Menon, M., Bolte, C. E., Faske, T. M., \& Eckert, A. J. (2018). The genomics of local adaptation in trees: are we out of the woods yet? Tree Genetics and Genomes, 14(2). doi: $10.1007 / \mathrm{s} 11295-017-1224-\mathrm{y}$

McCarroll, D., \& Loader, N. J. (2004). Stable isotopes in tree rings. Quaternary Science Reviews, 23(78), 771-801. doi: 10.1016/j.quascirev.2003.06.017

Mckown, A. D., Guy, R. D., Klápště, J., Geraldes, A., Friedmann, M., Cronk, Q. C. B., ... Douglas, C. J. (2014). Geographical and environmental gradients shape phenotypic trait variation and genetic structure in Populus trichocarpa. New Phytologist, 201(4), 1263-1276. doi: 10.1111/nph.12601

McKown, A. D., Guy, R. D., Quamme, L., Klápště, J., La Mantia, J., Constabel, C. P., ... Azam, M. S. (2014). Association genetics, geography and ecophysiology link stomatal patterning in Populus trichocarpa with carbon gain and disease resistance trade-offs. Molecular Ecology, 23(23), 57715790. doi: 10.1111/mec.12969

Mckown, A. D., Klápště, J., Guy, R. D., Geraldes, A., Porth, I., Hannemann, J., ... Douglas, C. J. (2014). Genome-wide association implicates numerous genes underlying ecological trait variation in natural populations of Populus trichocarpa. New Phytologist, 203(2), 535-553. doi: 10.1111/nph.12815

Meirmans, P. G. (2012). The trouble with isolation by distance. Molecular Ecology, 21(12), 2839-2846. doi: 10.1111/j.1365-294X.2012.05578.x

Muchero, W., Guo, J., DiFazio, S. P., Chen, J., Ranjan, P., Slavov, G. T., .. Tuskan, G. A. (2015). Highresolution genetic mapping of allelic variants associated with cell wall chemistry in Populus. BMC Genomics, 16, 24. doi: 10.1186/s12864-015-1215-z

Muchero, W., Sondreli, K. L., Chen, J.-G., Urbanowicz, B. R., Zhang, J., Singan, V., ... LeBoldus, J. M. (2018). Association mapping, transcriptomics, and transient expression identify candidate genes mediating plant-pathogen interactions in a tree. Proceedings of the National Academy of Sciences, 115(45), 11573-11578. doi: 10.1073/pnas.1804428115

Neale, D. B., \& Kremer, A. (2011). Forest tree genomics: growing resources and applications. Nature Reviews. Genetics, 12(2), 111-122. doi: 10.1038/nrg2931

Neale, D. B., \& Savolainen, O. (2004). Association genetics of complex traits in conifers. Trends in Plant Science, 9(7), 325-330.

Parchman, T. L., Gompert, Z., Mudge, J., Schilkey, F. D., Benkman, C. W., \& Buerkle, C. A. (2012). Genome-wide association genetics of an adaptive trait in lodgepole pine. Molecular Ecology, 21(12), 2991-3005. doi: 10.1111/j.1365-294X.2012.05513.x 
Pearce, D. W., Millard, S., Bray, D. F., \& Rood, S. B. (2006). Stomatal characteristics of riparian poplar species in a semi-arid environment. Tree Physiology, 26(2), 211-218.

Porth, I., Klápště, J., Skyba, O., Lai, B. S. K., Geraldes, A., Muchero, W., ... Mansfield, S. D. (2013). Populus trichocarpa cell wall chemistry and ultrastructure trait variation, genetic control and genetic correlations. The New Phytologist, 197(3), 777-790. doi: 10.1111/nph.12014

Pritchard, J. K., \& Di Rienzo, A. (2010). Adaptation - not by sweeps alone. Nature Reviews Genetics, 11(10), 665-667. doi: 10.1038/nrg2880

Rajora, O. P., Eckert, A. J., \& Zinck, J. W. R. (2016). Single-locus versus multilocus patterns of local adaptation to climate in eastern white pine (Pinus strobus, Pinaceae). PLoS ONE, 11(7), 1-26. doi: 10.1371/journal.pone.0158691

Rajora, O. P., Zsuffa, L., \& Dancik, B. P. (1991). Allozyme and leaf morphological variation of eastern cottonwood at the northern limits of its range in Ontario. Forest-Science, 37(2), 688-702. doi: S

Rellstab, C., Gugerli, F., Eckert, A. J., Hancock, A. M., \& Holderegger, R. (2015). A practical guide to environmental association analysis in landscape genomics. Molecular Ecology, 24(17), 4348-4370. doi: $10.1111 / \mathrm{mec} .13322$

Richardson, J. L., Urban, M. C., Bolnick, D. I., \& Skelly, D. K. (2014). Microgeographic adaptation and the spatial scale of evolution. Trends in Ecology and Evolution, 29(3), 165-176. doi: 10.1016/j.tree.2014.01.002

Richter, H. (1997). Water relations of plants in the field: some comments on the measurement of selected parameters. Journal of Experimental Botany, 48(306), 1-7. doi: 10.1093/jxb/48.1.1

Rubin, E. M. (2008). Genomics of cellulosic biofuels. 454(7206), 841-845.

Sack, L., \& Holbrook, N. M. (2006). Leaf hydraulics. Annual Review of Plant Biology, 57, 361-381. doi: 10.1146/annurev.arplant.56.032604.144141

Sack, L., \& Scoffoni, C. (2012). Measurement of leaf hydraulic conductance and stomatal conductance and their responses to irradiance and dehydration using the Evaporative Flux Method (EFM). Journal of Visualized Experiments : JoVE, (70), 1-7. doi: 10.3791/4179

Santiago, L. S., Goldstein, G., Meinzer, F. C., Fisher, J. B., Machado, K., Woodruff, D., \& Jones, T. (2004). Leaf photosynthetic traits scale with hydraulic conductivity and wood density in Panamanian forest canopy trees. Oecologia, 140(4), 543-550. doi: 10.1007/s00442-004-1624-1

Savolainen, O., Lascoux, M., \& Merilä, J. (2013). Ecological genomics of local adaptation. Nature Reviews. Genetics, 14(11), 807-820. doi: 10.1038/nrg3522

Savolainen, O., \& Pyhäjärvi, T. (2007). Genomic diversity in forest trees. Current Opinion in Plant Biology, 10(2), 162-167. doi: 10.1016/j.pbi.2007.01.011

Schuldt, B., Leuschner, C., Brock, N., \& Horna, V. (2013). Changes in wood density, wood anatomy and 
hydraulic properties of the xylem along the root-to-shoot flow path in tropical rainforest trees. Tree Physiology, 33(2), 161-174. doi: 10.1093/treephys/tps122

Scoffoni, C., McKown, A. D., Rawls, M., \& Sack, L. (2012). Dynamics of leaf hydraulic conductance with water status: quantification and analysis of species differences under steady state. Journal of Experimental Botany, 63(2), 643-658. doi: 10.1093/jxb/err270

Simmons, B. A., Loqué, D., \& Ralph, J. (2010). Advances in modifying lignin for enhanced biofuel production. Current Opinion in Plant Biology, 13(3), 313-320. doi: 10.1016/j.pbi.2010.03.001

Slavov, G. T., DiFazio, S. P., Strauss, S. H., den Nijs, J. C. M., Bartsch, D., \& Sweet, J. (2004). Gene flow in forest trees: Gene migration patterns and landscape modeling of transgene dispersion in hybrid poplar. In Introgression from Genetically Modified Plants into Wild Relatives (pp. 89-106). UK: CAB International.

Slavov, G. T., Leonardi, S., Adams, W. T., Strauss, S. H., \& DiFazio, S. P. (2010). Population substructure in continuous and fragmented stands of Populus trichocarpa. Heredity, 105(4), 348357.

Sork, V. L., Aitken, S. N., Dyer, R. J., Eckert, A. J., Legendre, P., \& Neale, D. B. (2013). Putting the landscape into the genomics of trees: Approaches for understanding local adaptation and population responses to changing climate. Tree Genetics and Genomes, 9(4), 901-911. doi: 10.1007/s11295013-0596-x

Sparks, J. E. D. P., \& Black, R. A. (1999). Regulation of water loss in populations of Populus trichocarpa: the role of stomatal control in preventing xylem cavitation. Tree Physiology, 19, 453459.

Sperry, J. S. (2000). Hydraulic constraints on plant gas exchange. Agricultural and Forest Meteorology, 104(1), 13-23. doi: 10.1016/S0168-1923(00)00144-1

Sperry, J. S., Hacke, U. G., \& Pittermann, J. (2006). Size and function in conifer tracheids and angiosperm vessels. American Journal of Botany, 93(10), 1490-1500. doi: 10.3732/ajb.93.10.1490

Stucki, S., Orozco-terWengel, P., Forester, B. R., Duruz, S., Colli, L., Masembe, C., ... Joost, S. (2017). High performance computation of landscape genomic models including local indicators of spatial association. Molecular Ecology Resources, 17(5), 1072-1089. doi: 10.1111/1755-0998.12629

Talbot, B., Chen, T. W., Zimmerman, S., Joost, S., Eckert, A. J., Crow, T. M., ... Manel, S. (2017). Combining genotype, phenotype, and environment to infer potential candidate genes. Journal of Heredity, 108(2), 207-216. doi: 10.1093/jhered/esw077

Tuskan, G. A. (1998). Short-rotation woody crop supply systems in the United States: What do we know and what do we need to know? Biomass \& Bioenergy, 14(4), 307-315.

Tuskan, G. A., Difazio, S., Jansson, S., Bohlmann, J., Grigoriev, I., Hellsten, U., ... Rouzé, P. (2006). The 
genome of black cottonwood, Populus trichocarpa (Torr. \& Gray). Science, 313(5793), 1596. doi: $10.1126 /$ science. 1128691

Vangestel, C., Eckert, A. J., Wegrzyn, J. L., St. Clair, J. B., \& Neale, D. B. (2018). Linking phenotype, genotype and environment to unravel genetic components underlying cold hardiness in coastal Douglas-fir (Pseudotsuga menziesii var. menziesii). Tree Genetics and Genomes, 14(1). doi: $10.1007 / \mathrm{s} 11295-017-1225-\mathrm{x}$

Wang, J., Ding, J., Tan, B., Robinson, K. M., Michelson, I. H., Johansson, A., ... Ingvarsson, P. K. (2018). A major locus controls local adaptation and adaptive life history variation in a perennial plant. Genome Biology, 19(1), 72. doi: 10.1186/s13059-018-1444-y

Wegrzyn, J. L., Eckert, A. J., Choi, M., Lee, J. M., Stanton, B. J., Sykes, R., ... Neale, D. B. (2010). Association genetics of traits controlling lignin and cellulose biosynthesis in black cottonwood (Populus trichocarpa, Salicaceae) secondary xylem. New Phytologist, 188(2), 515-532. doi: 10.1111/j.1469-8137.2010.03415.x

Wei, Z., Zhang, G., Du, Q., Zhang, J., Li, B., \& Zhang, D. (2014). Association mapping for morphological and physiological traits in Populus simonii. BMC Genetics, 15 Suppl 1(Suppl 1), S3. doi: 10.1186/1471-2156-15-S1-S3

Weighill, D., Jones, P., Shah, M., Ranjan, P., Muchero, W., Schmutz, J., ... Jacobson, D. (2018). Pleiotropic and Epistatic Network-Based Discovery: Integrated Networks for Target Gene Discovery. Frontiers in Energy Research, 6(May), 30. doi: 10.3389/fenrg.2018.00030

Weng, J.-K., Li, X., Bonawitz, N. D., \& Chapple, C. (2008). Emerging strategies of lignin engineering and degradation for cellulosic biofuel production. Current Opinion in Biotechnology, 19(2), 166172. doi: $10.1016 / j$.copbio.2008.02.014

Xiao, X. W., Xu, X., \& Yang, F. (2008). Adaptive Responses to Progressive Drought Stress in two Populus cathayana Populations. Silva Fennica, 42(5), 705-719.

Xie, C.-Y., Carlson, M. R., \& Ying, C. C. (2012). Ecotypic mode of regional differentiation of black cottonwood (Populus trichocarpa) due to restricted gene migration: further evidence from a field test on the northern coast of British Columbia. Canadian Journal of Forest Research, 42(2), 400 405. doi: 10.1139/x11-187

Xu, X., Yang, F., Xiao, X. W., Zhang, S., Korpelainen, H., \& Li, C. Y. (2008). Sex-specific responses of Populus cathayana to drought and elevated temperatures. Plant Cell and Environment, 31(6), 850860. doi: 10.1111/j.1365-3040.2008.01799.x

Yoder, J. B., Stanton-Geddes, J., Zhou, P., Briskine, R., Young, N. D., \& Tiffin, P. (2014). Genomic signature of adaptation to climate in Medicago truncatula. Genetics, 196(4), 1263-1275. doi: 10.1534/genetics.113.159319 
Yuan, J. S., Tiller, K. H., Al-Ahmad, H., Stewart, N. R., \& Stewart, C. N. (2008). Plants to power: bioenergy to fuel the future. Trends in Plant Science, 13(8), 421-429. doi:

10.1016/j.tplants.2008.06.001

Zanne, A. E., \& Falster, D. S. (2010). Plant functional traits-linkages among stem anatomy, plant performance and life history. New Phytologist, 185, 348-351. doi: 10.1111/j.14698137.2009.03138.x

Zhang, J., Yang, Y., Zheng, K., Xie, M., Feng, K., Jawdy, S. S., ... Muchero, W. (2018). Genome-wide association studies and expression-based quantitative trait loci analyses reveal roles of HCT2 in caffeoylquinic acid biosynthesis and its regulation by defense-responsive transcription factors in Populus. New Phytologist, 220(2), 502-516. doi: 10.1111/nph.15297

Zhou, L., Bawa, R., \& Holliday, J. A. (2014). Exome resequencing reveals signatures of demographic and adaptive processes across the genome and range of black cottonwood (Populus trichocarpa). Molecular Ecology, 23(10), 2486-2499. doi: 10.1111/mec.12752 
Chapter 2. Multitrit genome-wide association analysis of Populus trichocarpa identifies key polymorphisms controlling morphological and physiological traits

Note: This chapter is taken directly from my publication:

"Multitrait genome-wide association analysis of Populus trichocarpa identifies key polymorphisms controlling morphological and physiological traits”, New Phytologist 2019, 223: 293-309. Published 7 March 2019. DOI: 10.1111/nph.15777.

Authors: Hari B. Chhetri ${ }^{1}$, David Macaya-Sanz ${ }^{1}$,David Kainer ${ }^{2}$, Ajaya K. Biswal ${ }^{3,4}$, Luke M. Evans ${ }^{1}$, JinGui Chen ${ }^{2}$, Cassandra Collins ${ }^{5}$, Kimberly Hunt ${ }^{5}$, Sushree S. Mohanty ${ }^{3}$, Todd Rosenstiel ${ }^{6}$, David Ryno ${ }^{4}$, Kim Winkeler ${ }^{5}$, Xiaohan Yang ${ }^{2}$, Daniel Jacobson ${ }^{2}$, Debra Mohnen ${ }^{3,4}$, Wellington Muchero ${ }^{2}$, Steven H. Strauss $^{7}$, Timothy J. Tschaplinski ${ }^{2}$, Gerald A. Tuskan ${ }^{2}$, Stephen P. DiFazio ${ }^{1}$

${ }^{1}$ Department of Biology, West Virginia University, Morgantown, WV 26506

${ }^{2}$ Biosciences Division, Oak Ridge National Laboratory, Oak Ridge, TN 37831

${ }^{3}$ Department of Biochemistry and Molecular Biology, University of Georgia, Athens, GA 30602

${ }^{4}$ Complex Carbohydrate Research Center, University of Georgia, Athens, GA 30602

${ }^{5}$ ArborGen, Inc., 2011 Broadbank Ct., Ridgeville, SC, 29472, USA

${ }^{6}$ Department of Biology, Portland State University, Portland, OR 97207

${ }^{7}$ Department of Forest Ecosystems \& Society, Oregon State University, Corvallis, OR 97331

$H B C, D M-S, A K B, L M E, D K, T J T$, and SPD collected data and performed analyses; AKB, DM, CC, $L M E, K H, S S M, D R, K W$, and $X Y$ produced and characterized transgenics; J-G C, TR, DJ, WM, SHS, GAT, TJT, and SPD conceived and designed the study; HBC and SPD wrote the manuscript; DK, LME, $J-G C, D J, G A T, S H S$, and DM edited the manuscript. 


\subsection{Summary}

- Genome-wide association studies (GWAS) have great promise for identifying the loci that contribute to adaptive variation, but the complex genetic architecture of many quantitative traits presents a substantial challenge.

- We measured 14 morphological and physiological traits and identified SNP-phenotype associations in a Populus trichocarpa population distributed from California to British Columbia. We used whole genome re-sequencing data of 882 trees with more than 6.78 million SNPs, coupled with multitrait association to detect polymorphisms with potentially pleiotropic effects. Candidate genes were validated with functional data.

- Broad-sense heritability $\left(H^{2}\right)$ ranged from 0.30 to 0.56 for morphological traits and 0.08 to 0.36 for physiological traits. A total of 4 and 20 gene models were detected using the single trait and multitrait association methods, respectively. Several of these associations were corroborated by additional lines of evidence, including co-expression networks, metabolite analyses, and direct confirmation of gene function through RNAi.

- Multitrait association identified many more significant associations than single trait association, potentially revealing pleiotropic effects of individual genes. This can be particularly useful for challenging physiological traits like water-use efficiency or complex traits like leaf morphology, for which we were able to identify credible candidate genes by combining multitrait association with gene co-expression and co-methylation data.

\subsection{Non-technical summary}

We used markers from across the entire genome together with combinations of related traits to identify candidate genes that potentially control important adaptive traits in the black cottonwood tree. Several of these genes were corroborated by functional analysis, including a possible transcriptional regulator of drought responses, as well as a gene involved in cell wall composition that affects leaf size. This study provides a framework for direct functional annotation of genes, which remains a major challenge for recalcitrant model organisms like forest trees. 


\subsection{Introduction}

A long-standing question in evolutionary biology is the role of selection in shaping the spatial and temporal patterns of phenotypic variation (Weigel \& Nordborg, 2015). In the era of genomics, it is now possible to identify the molecular mechanisms underlying phenotypic variation on the landscape. Owing to their wide geographical distribution and climatic gradients, forest trees are an excellent model system for testing how genetic drift, and selection affect genetic variation within a species (Neale \& Savolainen, 2004; Ingvarsson \& Street, 2011; Neale \& Kremer, 2011; Ingvarsson et al., 2016). Forest trees generally have large effective population sizes, extensive gene flow, high genetic variation, and local adaptation (Neale \& Savolainen, 2004; González-martínez et al., 2006; Ingvarsson \& Street, 2011). About 31\% of the total land area on earth is occupied by forests, which are of great ecological and economic importance (MacDicken et al., 2016), so understanding the factors affecting variation in traits that are important for environmental adaptation is of utmost importance, particularly in the context of rapidly changing climates (Aitken et al., 2008).

Association genetics has emerged as a major tool for identifying the genomic regions underlying traits of interest (Ingvarsson \& Street, 2011). Using natural populations that have undergone many generations of recombination between ancestral haplotypes allows identification of the genomic region affecting a trait at fine scale. Nevertheless, one of the major downsides of association mapping is the requirement of large numbers of loci and individuals (Visscher et al., 2017). With recent advances in sequencing technologies, acquiring genomic data at a whole-genome scale has become much more feasible. Nevertheless, despite the high heritability of many morphological traits, only a small proportion of heritability is explained by single nucleotide polymorphisms (SNPs) in most GWAS analyses, suggesting insufficient statistical power (Solovieff et al., 2013). In fact, power analyses indicate that most association studies in forest trees are orders of magnitude too small to detect the effects of alleles of small effect and low frequency (Visscher et al., 2017), which collectively account for a large fraction of the heritability of complex traits (Boyle et al., 2017).

While increasing sample size of GWAS populations is clearly desirable, it is costly and, in some cases, may not be feasible. Alternatively, approaches that can improve the power of GWAS from the same inputs can be used. One approach is to use gene and pathway-based analysis, where GWAS is performed on a set of SNPs or genes (Kim et al., 2016). Another option is to take a multitrait approach, where GWAS is performed with multiple related traits combined in a multivariate framework. Recently, the latter approach has gained some popularity because it offers substantial increase in power compared to the standard univariate approach (Porter \& O'Reilly, 2017). One of the big advantages of multitrait GWAS is that missing information in one of the phenotypes in the multitrait set can be complemented by the other phenotypes (Ritchie et al., 2015). The increased power of multitrait GWAS depends in part on 
correlation among traits (Porter \& O'Reilly, 2017) and the combination of weak genetic effects across the traits (Casale et al., 2015). Multitrait GWAS also takes advantage of pleiotropic effects of polymorphisms, thereby increasing statistical power even when the traits have low correlation (Broadaway et al., 2016; Hackinger \& Zeggini, 2017). Finally, unlike analyses based on principal components, multitrait GWAS effectively captures indirect genetic effects whereby a SNP affects one phenotype through its effects on a functionally-related phenotype (Stephens, 2013; Porter \& O'Reilly, 2017).

Here we used the model species $P$. trichocarpa to explore the utility of multitrait GWAS to detect genetic variants controlling adaptive traits. The genus Populus has a wide distribution in the northern hemisphere and are dioecious, wind pollinated, and highly heterozygous. Populus are also fast growing, easy to propagate, and demonstrate interspecific hybrid vigor, all of which makes the genus a model system with high economic potential for production of forest products and biofuels (Jansson \& Douglas, 2007; Rubin, 2008). P. trichocarpa is from section Tacamahaca (Eckenwalder, 1996) and is distributed from central California to northern British Columbia (BC). It is the first tree genome to be sequenced (Tuskan et al., 2006) and a tremendous amount of genetic resources are available, including abundant transcriptomes (Sjödin et al., 2009; Geraldes et al., 2011; Zhang et al., 2018b) and re-sequencing data (Slavov et al., 2012; Evans et al., 2014), as well as multiple association populations in replicated plantations (Evans et al., 2014; McKown et al., 2014c; Holliday et al., 2016). From these studies, and others, it has been shown that climate plays a major role in shaping genetic variation and driving selection within this species. We show here that multitrait GWAS is a substantially more powerful approach than single trait GWAS in identifying molecular determinants of quantitative traits, though much remaining heritable variation remains to be identified.

\subsection{Materials and methods}

\subsubsection{Plantation establishment and phenotyping}

The $P$. trichocarpa association population consists of 1084 trees collected from natural populations in western Washington, Oregon, California, and BC (Figure 2.1). The trees were clonally propagated from stem cuttings and planted in a common garden in Corvallis, OR in July of 2009 (Evans et al., 2014). The plantation consists of 3 blocks in a completely randomized design and the trees were planted at $2 \mathrm{~m} \times 3 \mathrm{~m}$ spacing. The plantation was coppiced in December of 2010 and again in December of 2013. Coppiced plants were allowed to re-sprout and grow for one season, after which they were pruned to a single leader in January of the following year (2012 and 2015, respectively).

In December 2013, 759 trees were sampled for carbon isotope analysis. Wood cores (12 $\mathrm{mm}$ ) were taken from breast height of the tree and the 2012 growth ring was selected for analysis. Cross section of the wood tissue representing the entire growth ring (about 1.2 to $1.8 \mathrm{mg}$ ) representing early, intermediate and 
late wood were sampled. The wood samples were oven-dried at $65^{\circ} \mathrm{C}$ for at least $72 \mathrm{~h}$, weighed and wrapped in a tin capsule before sending to the Appalachian Ecology Lab in Frostburg, Maryland for analysis. Carbon isotope composition $\left(\delta^{13} \mathrm{C}\right)$ was estimated as follows:

$$
\delta^{13} \mathrm{C}=\left(\frac{R_{\text {sample }}}{R_{\text {standard }}}-1\right) \times 1000,
$$

where, $R_{\text {sample }}$ and $R_{\text {standard }}$ are the ${ }^{13} \mathrm{C} /{ }^{12} \mathrm{C}$ ratios in a sample and standard, respectively.

In July of 2014 leaf characteristics were measured for 1056 trees (one complete block plus a subset of replicates, Table 2.1). The first and second fully expanded leaves (counting from the apex) were collected from a branch receiving full sunlight. One of the leaves was used for measuring petiole length and diameter with a digital caliper and then scanned using a hand-held scanner. Images were analyzed to estimate leaf area, leaf length, leaf width and leaf perimeter using imageJ software (Shindelin et al., 2015). Dry weights were determined for the same leaves for estimates of specific leaf area (SLA). Leaf chlorophyll (SPAD) was assessed using a SPAD 502 Plus meter (Spectrum Technologies) with an average of 3 replicate measures on leaf section. Abaxial stomatal density was measured by applying clear nail polish to the broadest part of the leaf close to the midrib. A clear piece of tape was then used to capture an imprint of the epidermal leaf surface. These were mounted on slides and the number of stomata in $1 \mathrm{~mm}^{2}$ area in 4 random fields was counted at 400x magnification. Pre-dawn leaf water potential was measured for 964 trees using the cut petiole method (Scholander et al., 2016). Measurements were made on a fully-expanded leaf from the middle of the canopy. Leaves were collected between the hours of 2 $\mathrm{AM}$ and $5 \mathrm{AM}$ and a pressure bomb was used in the field to measure the pressure of $\mathrm{N}_{2}$ gas required to force sap from the cut petiole. Height was measured following the 2015 growing season.

\subsubsection{Statistical analyses}

All measurements were checked for recording errors and outliers were removed. Data were checked for normality. The phenotypic values were adjusted for any within garden microsite variation using Thin Plate Spline (tps) regression using the Tps function of the fields package in R. Using tpsadjusted phenotypic values, broad-sense heritabilities were estimated for all traits using the genotypes with replicate clonal measurements. Variance components were estimated by fitting the model with the lmer and ranef functions of the lme4 package in $\mathrm{R}$, with genotype as a random effect, and error estimated from the residuals of the model:

$$
H^{2}=\frac{\sigma_{G}^{2}}{\sigma_{G}^{2}+\sigma_{E}^{2}}
$$

Genetic correlation between traits was estimated using Best Linear Unbiased Predictors (BLUPs) from the same model. Using the clonal tps-adjusted values, the Pearson correlation was performed using the cor function of the stats package in $\mathrm{R}$. The prcomp function of the ggbiplot package in $\mathrm{R}$ was used to 
estimate the relationships of the phenotypes using linear combinations (Principal Components) of the original phenotypic values. Leaf Water Potential was not included in the PCA due to low heritability.

\subsubsection{Genotypic data}

Preparation of the genotypic data was as described in Evans et al. (2014) and Weighill et al.(2018). Briefly, whole-genome re-sequencing was performed for 1053 trees using Illumina genetic analyzers at the DOE Joint Genome Institute. Pairwise relatedness was calculated using GCTA (Yang et $a l ., 2011)$, taking population structure into account. Trees related more closely than first cousins were removed from the analyses. The remaining 882 individuals were used for all subsequent analyses. A genetic relationship matrix was estimated for the remaining trees using GEMMA, and used as a covariate in GWAS analyses. Principal Components (PCs) of all resequencing data were estimated using smartpca from EIGENSOFT v6.1.4 and the first 60 PCs were selected as potential covariates for the association tests. Stepwise regression using the step function with default selection criteria (i.e. both backward and forward selection) of the MASS package in R was used for selecting PCs that were significantly associated with each phenotype or group of phenotypes. All significant PCs were used as covariates for GWAS (Supplementary Table S2.1). Finally, SNPs with minor allele frequency $\leq 0.05$ and markers with severe departures from Hardy-Weinberg expectations were removed.

\subsubsection{Test for association}

Association tests were performed using GEMMA (Zhou \& Stephens, 2012, 2014). Phenotypic BLUPs, genetic relationship matrix, significant PC axes of the genotypic data and 6,781,211 SNPs (remaining SNPs after filtering with MAF less than 0.05) were used for the association test. Single trait GWAS was run for 14 phenotypes (Table 2.1). The tested model was:

$y=W \alpha+x \beta+u+\epsilon$,

where $y$ is an $n$-vector of phenotypic BLUP values, where $n$ is the number of individuals tested; $W$ is an $n \times c$ matrix of covariates; $\alpha$ is a $c$-vector of corresponding coefficients, where $c$ is the number of principal coordinate axes used; $x$ is an $n$-vector of marker genotypes, $\beta$ is the effect size of the marker, $u$ is an n-vector of random effects that includes a relatedness matrix and $\epsilon$ is an $n$-vector of errors.

\subsubsection{Trait selection for multitrait GWAS}

Multitrait combinations were created based on genetic correlations among phenotypes as well as hypothesized structural and functional relationships of the traits. The latter can be important even in the absence of genetic correlations (Stephens, 2013). Pairwise genetic correlations were performed (Figure 2.2; Supplementary Table S2.2) and the functional relationships were assessed through relevant literature for the phenotypes before forming 12 multitrait sets (Table 2.2). For example, leaf area, leaf dry weight, leaf length and leaf width were combined to form a multitrait set because these traits are highly intercorrelated and represent the leaf as a structural unit. Likewise, tree height, leaf area and petiole length 
were combined because the traits are inter-correlated and all affect interception of photosynthetically active radiation. We also combined traits that did not have high genetic correlations, but presumably had functional relationships. For example, we combined carbon isotope composition, leaf water potential and stomatal density because these traits together provide a broader picture of water-use efficiency (WUE), abiotic stress and gas exchange in plants. Pre-dawn leaf water potential is a measure of water retaining capacity of the plants. Measurement of $\delta^{13} \mathrm{C}$ composition in wood provides a measure of integrated WUE. Higher composition of $\delta^{13} \mathrm{C}$ is related to lower carbon isotope discrimination, which in turn is related to high water use efficiency. The gas exchange process in plant leaves is regulated by stomata in the leaves and the number and density and number of stomata is key for this mechanism. Multitrait association was conducted with GEMMA using the same model as for single trait associations, except $y$ is an $n \times d$ matrix of $d$ phenotypes for $n$ individuals.

\subsubsection{Analyses of association results}

Determining a significance cutoff is one of the biggest challenges for high-dimension analyses like GWAS (Sham \& Purcell, 2014). Here we have chosen a uniformly conservative approach to facilitate comparisons among GWAS methods. We used a $P$-value cutoff $(\alpha \leq 0.05)$ based on the Bonferroni correction criterion of $7.37 \times 10^{-9}$ and a more liberal $P$-value cutoff of $1 \times 10^{-7}$ to identify suggestive associations. These were later cross-referenced to other sources of evidence to highlight robust associations (see below). For the purpose of summarizing the results, significant SNPs within $10 \mathrm{~kb}$ of one another were merged and counted as a single significant locus. Gene models that were closest to significant SNPs were identified based on v3 of the P. trichocarpa genome. Annotation information was obtained from Phytozome, including expression level in different plant tissues and annotations of putative gene functions (Goodstein et al., 2012). Percentage of variance explained (PVE) by SNPs was estimated using the formula in Shim et al. (2015).

\subsubsection{Network analysis}

To gain further insight into possible biological functions of candidate genes identified by the GWAS analysis, we examined the position of the genes in networks constructed for the same population that was used in this paper. The networks were based on the following: gene co-expression using the JGI Plant Gene Atlas for P. trichocarpa (https://phytozome.jgi.doe.gov); GWAS of metabolite profiles determined by GC-MS (Tschaplinski et al., 2012), and methylation data for multiple tissues in $P$. trichocarpa (Vining et al., 2012). Details of the underlying data and network construction can be found in Weighill et al. (2018). Briefly, candidate genes identified from single and multitrait GWAS were used as a seed to identify subnetworks that were potentially related to the gene. A merged network was created by combining metabolites at False Discovery Rate (FDR) of 0.1, significant GWAS SNPs linked to the 
corresponding phenotypes and the co-expression and co-methylation subnetworks. The networks were visualized in Cytoscape version 3.6.1 (Shannon et al., 2003).

\subsubsection{Functional analysis of the GAUT9 candidate gene}

Detailed functional characterization was conducted for one of the genes highlighted by the multitrait GWAS and network analyses presented here in order to provide experimental validation of the functional roles inferred by our analyses. A $123 \mathrm{bp}$ fragment comprising portions of the coding region and 3'-untranslated region of Potri.004G111000 (PtGAUT9.1) was amplified via PCR from a P. trichocarpa cDNA library using the following gene specific primers: PtGAUT9.1-F (CACCCCCGGGTTTGGCCTTTAGACGAATTCC) and PtGAUT9.1-R (TCTAGAGTGACAACTAATGATCGGATCCA). The fragment was cloned into an RNAi cassette and transferred to a binary vector for Agrobacterium-mediated transformation of the $P$. deltoides clone WV94, as previously described (Biswal et al., 2015, 2018a). Measurement of leaf traits was carried out on 10 plants each of 3-month-old WT, empty vector control, and PdGAUT9.1-KD lines. RNA isolation and quantitative RT-PCR were performed as previously described (Biswal et al., 2015, 2018a). Briefly, total RNA was isolated using an RNeasy Plant Mini Kit (Qiagen, Valencia, CA). The primers PdGAUT9.1-qRT-F (GTGCTTGGCCTCGGATATAA) and PdGAUT9.1-qRT-R (GAAACATGAAACCTTGGCTTGA) were used to amplify the target gene, PdGAUT9.1. The closelyrelated PdGAUT9.2 gene was also evaluated to demonstrate the specificity of down-regulation using the primers qRT-F (GCGGCATCAATGGTGGATTA) and PdGAUT9.2-qRT-R (TTCTATTCCTCGCCACTCTCTC).

We also evaluated the impacts of the associated polymorphism on gene expression in the association population using RNAseq data from developing xylem, as described by Zhang et al. (2018b). Briefly, normalized gene expression was determined using TopHat2, Cufflinks, and Featurecounts, and normalized via DESeq2, followed by Pearson's correlation with the genotype.

\subsection{Results}

\subsubsection{Physiological and morphological trait variation}

Broad-sense heritabilities ranged from 0.297 to 0.561 for morphological traits, and from 0.080 to 0.363 for physiological traits (Table 2.1). Pre-dawn leaf water potential had low broad-sense heritability that was not significantly different from 0 (Table 2.1). Although TPS regression was used to correct for microsite variation, physiological traits like pre-dawn leaf water potential appear to be very sensitive to environmental conditions and the micro-climatic conditions at the time of sampling.

Most morphological traits were highly inter-correlated whereas physiological traits were generally not inter-correlated, which is consistent with expectations due to the high measurement error for the latter (Figure 2.2; Supplementary Table S2.2). We performed Principal Component Analysis (PCA) to 
further explore the relationships among traits within the population. The first principal component (PC1) explained more than $47 \%$ of the total variation. PC1 and PC2 together explained $61 \%$ of the total variation. Morphological traits were positively weighted toward the PC1 axis (Figure 2.3), which also shows a slight negative correlation with the latitude of the provenance $(\mathrm{r}=-0.17, P \leq 0.001)$. Specific leaf area and stomatal density were negatively weighted for PC2, while SPAD was positively weighted along this axis (Figure 2.3; Supplementary Table S2.3). PC2 generally separated the Columbia population from $\mathrm{BC}$ and the core subpopulations (Figure 2.3). Most morphological traits were correlated with the latitude of origin (Supplementary Table S2.4).

\subsubsection{SNP-trait associations}

We conducted single trait GWAS with 6.78 million SNPs for the 14 morphological and physiological traits. We did not identify any SNP that passed Bonferroni correction $\left(P<7.37 \times 10^{-9}\right)$. However, we identified a total of 4 SNPs (Table 2.1; Figure 2.4a) that passed a suggestive association $P$ value cutoff of $1 \times 10^{-7}$. These associated SNPs were within or close to four $P$. trichocarpa gene models (Table 2.3). PVE of significant SNPs ranged from 3.45\% to $4.35 \%$ (Supplementary Table S2.5), though this is likely to be inflated since it is estimated in the discovery population.

Multitrait GWAS for a total of 12 sets of traits identified 5 SNPs that passed the Bonferroni correction $P$-value cutoff and a total of 32 SNPs that passed the suggestive association $P$-value cutoff of $1 \times 10^{-7}$ (Figure 2.4b; Table 2.4). These SNPs were within or close to 22 P. trichocarpa gene models (Table 2.4). PVE of these SNPs ranged from $0.0003 \%$ to $4.35 \%$ for the individual traits comprising the multitrait set (Supplementary Table S2.5).

To facilitate the presentation, we divided the multitrait association results into the following three categories based on the correspondence of the multitrait and the single trait results: 1) multitrait GWAS with increased power for the same (or nearby) SNP positions as in the single trait GWAS (Figures 2.5a,b; Supplementary Figure S2.1); 2) multitrait GWAS with increased power, but with different genomic positions than the single trait GWAS (Figures 2.5c,d; Supplementary Figures S2.2 \& S2.3) multitrait GWAS with reduced power for some loci, but with the same (or nearby) SNP positions as in the single trait GWAS (Figures 2.5e,f; Supplementary Figure S2.3). Each category contained 4 multitrait combinations. QQ plots showed a clear improvement for multitrait association compared to the corresponding single trait association (Supplementary Figures S2.4-S2.6).

To provide further evidence for the involvement of the associated SNPs in trait variation, we integrated our GWAS results with other independent datasets, including leaf metabolite levels, gene expression and tissue-specific methylation. Three genes that were significantly associated with carbonisotope, leaf area and stomatal density (CI-LA-SD) showed interesting linkages to co-expressed genes and/or metabolites, including a potential regulatory network mediated by YABBY transcription factors, 
and a possible regulatory network that is associated with phenolic composition (Figure 2.6;

Supplementary Tables S2.6 \& S2.7). Similarly, the multitrait associations for leaf shape (leaf area-leaf dry weight-leaf length-leaf width, and specific leaf area-leaf aspect ratio) were linked by association with common candidate genes and revealed two large co-expression networks as well as a group of coexpressed enzymes that affect cell wall characteristics (Figure 2.7).

\subsubsection{Direct evidence of the role of GAUT9 in determining leaf area in Populus}

One of the genes associated with leaf morphology (LA-LD-LL-LW) was Potri.004G111000, annotated as galacturonosyltransferase 9 (GAUT9). The polymorphism was $1.9 \mathrm{~kb}$ downstream of the end of the predicted stop codon (Supplementary Table S2.6). The next closest gene is nearly $33 \mathrm{~kb}$ away from the SNP, so Potri.004G111000 is the most likely gene to be impacted by this polymorphism. Consistent with this, the associated polymorphism was significantly correlated with the expression of Potri.004G111000 in developing xylem samples from the association population (Supplementary Figure S2.7a, $\mathrm{r}=0.169, P<0.001$ ), but not in fully expanded leaves (Supplementary Figure S2.7b, $\mathrm{r}=0.035$, ns). Lack of correlation in leaves is likely due to sampling of the wrong developmental stage, but this requires further investigation.

In the process of studying the role of Potri.004G111000 in the recalcitrance of $P$. deltoides xylem, multiple $P$. deltoides RNAi lines were generated and leaf characteristics of wild type, vector control and three RNAi PdGAUT9.1-KD lines were compared (Figure 2.8). Reducing the GAUT9.1 transcript level by $51-60 \%$ (Figure $2.8 \mathrm{c}$ ) resulted in a $43-66 \%$ increased leaf length and leaf width at all developmental time points analyzed in 3-month old greenhouse grown plants (Figures 2.8d-f).

\subsection{Discussion}

Identification of the genetic underpinnings of adaptive trait variation has been an elusive goal of forest tree research for more than a century (Wheeler et al., 2015). Such efforts have been greatly enhanced in the age of genomics, which potentially enables identification of sequence variants controlling heritable variation. The genus Populus has been a focus of much of this effort due to the tremendous investment in genetic and genomic resources in recent years (Jansson \& Douglas, 2007). Previous studies have demonstrated that $P$. trichocarpa contains substantial heritable variation that has been shaped by the combined effects of demographic history and selection (Slavov et al., 2012; Evans et al., 2014; Geraldes et al., 2014; Holliday et al., 2016). However, studies focused on GWAS of individual complex traits have mostly failed to uncover variants that control a majority of the genetic variation in P. trichocarpa (Evans et al., 2014; Geraldes et al., 2014; McKown et al., 2014b, 2018), most likely due to a lack of power to detect variants of small effect and/or low allele frequency (Visscher et al., 2017). Here we attempt to compensate for these problems by using a larger GWAS population and by performing multitrait GWAS 
coupled with multiple lines-of-evidence to support the roles of marginally-associated loci in the target phenotypes.

\subsubsection{Morphological and physiological trait correlations and influence of geography}

Largely consistent with previous studies (Evans et al., 2014; McKown et al., 2014a; Holliday et al., 2016), correlations of morphological and physiological traits with latitude in our study suggests that the variation in adaptive traits in $P$. trichocarpa is partly driven by geography. There was a negative correlation of tree height with latitude, indicating that northern provenances grew poorly in our test site. Many leaf traits were also correlated with height as well as latitude, so the functional relationships among these traits cannot be readily discerned. Based on the correlation coefficients, leaves became smaller and thicker with low abaxial stomatal density and high chlorophyll content for trees from higher latitudes. Several other Populus studies in common gardens have reported higher nitrogen content, stomatal conductance and photosynthetic assimilation in northern trees (Gornall \& Guy, 2007; McKown et al., 2014a; Soolanayakanahally et al., 2015; Elmore et al., 2017; Momayyezi \& Guy, 2017). Furthermore, Gornall \& Guy (2007) and McKown et al. (2014b) found a negative correlation between abaxial stomata density and latitude, but they further indicated that the northern $P$. trichocarpa trees were amphistomatous, with adaxial stomata density increasing with the latitude. Most trees used in our study lacked adaxial stomata, likely reflecting the more southerly distribution of our collection (data not shown).

For the most part we found no clear relationships between wood $\delta^{13} \mathrm{C}$ and leaf traits or latitude of origin. This was unlikely to be due to excessive experimental error because these traits all showed significant broad sense heritability. This is consistent with other published reports for Populus. For example, a previous field study of $P$. trichocarpa revealed no correlation between $\delta^{13} \mathrm{C}$ of wood and location of origin for a wide variety of morphological and physiological traits (McKown et al., 2014a). However, in a greenhouse study of $P$. trichocarpa, intrinsic WUE was correlated with photosynthetic assimilation and leaf mass area (Momayyezi \& Guy, 2017). Similarly, P. balsamifera showed a positive correlation of wood and leaf $\delta^{13} \mathrm{C}$ with latitude in a greenhouse study (Soolanayakanahally et al., 2009) and no correlation with latitude in a field study (Soolanayakanahally et al., 2015). Monclus et al. (2009) found a correlation between $\delta^{13} \mathrm{C}$ and productivity traits (fresh biomass, height and circumference) for $P$. deltoides $\times P$. trichocarpa hybrids but no correlation of leaf $\delta^{13} \mathrm{C}$ and productivity for $P$. deltoides $\times P$. nigra hybrids (Monclus et al., 2005). The variability in these results may be due to the effect of environments of the common gardens or variation in the genotypic responses to drought (Soolanayakanahally et al., 2015). 


\subsubsection{Enhanced power with multitrait GWAS}

Despite a relatively large sample size and the use of whole genome SNP data, single trait GWAS for 14 traits revealed only 4 loci with suggestive associations, and collectively these explain only a very small percentage of variance in the phenotypes. In contrast, multitrait GWAS for 12 combinations of a subset of these traits identified 32 SNPs in or near 20 genes. It appears that the overall power of the analysis was moderately improved for most trait combinations compared to the corresponding single trait GWAS. The power of multitrait GWAS depends on multiple factors, including SNP effect size, direction of effect (positive vs. negative), percentage of variance explained (PVE) by the SNP, and trait correlations (Zhou \& Stephens, 2014; Porter \& O’Reilly, 2017). Additionally, because many of our traits were measured without replication, the multitrait analyses may have provided more accurate estimates of the underlying phenotypes due to covariance of some of the traits. Although we used the presumed functional relationship and the correlations of the traits to form the multitrait sets for this study, we cannot determine the relative impacts of these factors in these specific analyses, though this has been explored elsewhere through simulation studies (Zhou \& Stephens, 2014; Porter \& O'Reilly, 2017). Nevertheless, we can gain some insights by examining the PVE of the significant SNPs from multitrait GWAS in the corresponding single trait analyses to indirectly infer the contribution of each of the component traits. In the following section we explore this using case studies from each of the three categories defined above.

\subsubsection{Category 1}

This category includes multitrait GWAS with increased power for the same (or nearby) SNP positions as in the single trait GWAS. The multitrait GWAS that includes carbon isotope, leaf area and stomatal density (CI-LA-SD) well represents this category (Figure 2.5a). The multitrait GWAS detected 4 suggestive SNPs, each of which was in approximately the same position as a non-significant peak from at least one of the corresponding single trait GWASs. Examination of the effect plot for the SNP with the highest significance (Figure 2.5b) suggests that the multitrait GWAS may be capturing a pleiotropic effect in this case, since two of the traits, $\delta^{13} \mathrm{C}$ and stomatal density, both have higher means for homozygotes for the major allele compared to the other genotype classes. A similar pattern is evident for the peaks on Chr01 and Chr02, though in these cases the allelic effects are in opposite configurations for leaf area and stomatal density (Supplementary Figure S2.8), possibly reflecting a weak negative correlation between these traits.

Examination of provisional annotations and direct functional linkages for the genes closest to the associated SNPs provides further insight into the possible mechanisms by which these loci affect these three traits. For example, Potri.002G145100, a putative YABBY-1 related axial regulator, was coexpressed with 12 other genes, including another YABBY-5 transcription factor and a WUSCHELrelated homeobox gene family member, possibly representing a large regulatory network (Figure 2.6). 
Another possible regulatory network contained Potri.001G411800, an EF-Hand Calcium Binding Domain protein. In $P$. trichocarpa, Potri.001G411800 has moderate expression in early and late dormant bud, early male and female development, root tip, young, immature and first fully expanded leaves (Goodstein et al., 2012). This gene was co-expressed with 6 other genes, including another EF-hand family protein, Potri.011G129100. Calcium is an important second messenger in eukaryotes and has important roles in cell signaling and response to biotic and abiotic stresses and developmental cues (Sanders et al., 2002; Chen et al., 2015; Ranty et al., 2016; Zhu, 2016; Edel et al., 2017). The EF-hand motif is the most common and highly conserved calcium-binding motif (Lewit-Bentley \& Réty, 2000; Zeng et al., 2017).

This co-expression network provides further evidence that Potri.001G411800 is involved in responses to abiotic stress. It is co-expressed with a late embryogenesis abundant (LEA) hydroxyprolinerich glycoprotein (Potri.009G158900), a group that has a major role in responses to drought, salinity and, osmotic and temperature related stresses (Gao \& Lan, 2016; Magwanga et al., 2018). Potri.001G411800 is also associated with 10 different metabolites in the same population, including several that are related to plant development and stress responses (Figure 2.6). For example, at least five of the metabolites are identified as flavonoids or flavonoid glycosides, including caffeoyl-quercetin glycoside, coumaroyl caffeoyl glycoside, and catechol glycoside. Flavonoids are known to have antioxidant properties that are induced under abiotic and biotic environmental stresses (Hernández et al., 2009). Quercetin glycosides also play an important role in plant growth and development (Parvez et al., 2004). More importantly, they are known to have a role in osmotic adjustment wherein the deleterious effect of water deficit is minimized by the active accumulation of solutes such as glycosides and phenolics as a response to drought (Tschaplinski et al., 2019).

\subsubsection{Category 2}

This category includes cases in which the multitrait GWAS had increased power, but the associated loci did not overlap with peaks in the single trait GWAS. The multitrait set that includes leaf area, leaf dry weight, leaf length and leaf width (LA-LD-LL-LW) well represents this category (Figure $2.5 \mathrm{c})$. We detected 10 significant SNPs in the multitrait GWAS compared to none in the corresponding single trait GWAS, and the peaks were largely non-overlapping. The locus with the highest association in the multitrait analysis showed similar patterns of genotypic means for all four traits, consistent with an additive effect (Figure 2.5d). Most of the other cases had low minor allele frequency and high variation among phenotypes for homozygotes for the minor allele (Supplementary Figure S2.9; Supplementary Table S2.5). This is likely a case where joint estimation of the variances provided more power to detect differences among the genotypic classes, resulting in significant multitrait associations for loci that showed no association with the individual component traits. 
This case study also provides an example of the use of multiple lines of evidence to provide further support for relatively weak associations (Weighill et al., 2018). For example, the SNP Chr04_9996091 had a $P$-value of $4.72 \times 10^{-8}$, which does not pass a strict FDR with $\alpha=0.05$, so we classified this as a "suggestive" association. However, we have multiple lines of evidence that the closest gene model, Potri.004G111000, is involved with leaf development. This gene encodes a putative galacturonosyltransferase that is moderately expressed in the first fully expanded leaf, young and immature leaves, and pre-dormant and fully open vegetative buds (Goodstein et al., 2012). The closest Arabidopsis thaliana homolog of this gene, AT3G02350, encodes a gene annotated as galacturonosyltransferase 9 (GAUT9), for which enzyme activity has not yet been established. GAUT9 belongs to the GAUT gene family of proven and putative pectin homogalacturonan (HG) galacturnosyltransferases (Sterling et al., 2006; Atmodjo et al., 2013; Biswal et al., 2018b; Voiniciuc et al., 2018). Down-regulation of the PdGAUT9.1 gene caused increased leaf length and width in both developing and mature leaves of greenhouse-grown $P$. deltoides, confirming the role of the gene in leaf development in Populus.

Other GAUT genes have also been shown to affect cell wall properties and leaf size in Populus. Downregulation of a GAUT12 homolog in P. deltoides showed decreased xylan and pectin content in the cell wall and increased biomass yield (Biswal et al., 2015), while over-expression showed a reduction in overall plant productivity and resulted in smaller leaves, reduced xylem cell numbers and size, and an increase in in the amount of xylose and galacturonic acid in the cell wall (Biswal et al., 2018b). Downregulation of GAUT4 in P. deltoides resulted in decreased pectic homogalacturonan and rhamnogalacturonan II and increased plant height, diameter, leaf area, and biomass (Biswal et al., 2018a). Additional evidence supporting increased leaf growth with decreases in pectin is provided by reports of increased expansion of Arabidopsis rosette leaves resulting from overexpression of polygalacturonase, an enzyme that degrades pectic homogalacturonan (Rui et al., 2017).

Co-expression analysis lends further support for the involvement of this gene in cell wall biosynthesis. Potri.004G111000 (GAUT9) was co-expressed with 17 other gene models and one metabolite that are cell-wall related (Figure 2.7; Supplementary Tables S2.6 \& S2.7). For example, Potri.004G123500, is annotated as a Uridine diphosphate (UDP) glycosyltransferase (UGT) superfamily. In P. trichocarpa, this gene had moderate expression in pre-, early and late dormant buds, young and immature and first fully expanded leaves, and stem nodes and internodes (Goodstein et al., 2012). Another gene, Potri.010G102300, encodes a xyloglucan endotransglucosylase that is a member of the Glycoside Hydrolase Family 16 and which is also expected to affect cell wall properties (Nishikubo et al., 2011; Yang et al., 2014). 
Two other genes with suggestive associations to leaf morphology characteristics were coexpressed with a large number of other genes with potential regulatory functions. Potri.008G144100 is similar to the AAA-ATPase subunit of the 26S proteasome complex, and it was co-expressed with eight other genes with putative roles in protein degradation or synthesis, plus an ATP-dependent caseinolytic protease (Potri.018G018800) potentially involved in lipid processing (Figure 2.7; Supplementary Table S2.7). Another gene associated with leaf morphology, Potri.001G173900, encodes a Tudor-like RNAbinding protein with conserved ENT and Agenet domains. There is emerging evidence that the latter domain may be involved in transcriptional regulation in Arabidopsis (Zhang et al., 2018a). This gene was co-expressed with 49 other genes with putative roles in protein degradation or RNA regulation, as well as nine genes with annotations related to carbohydrate metabolism (Supplementary Tables S2.6,S2.7). These two genes are excellent candidates as master regulators of leaf morphology, possibly mediated by cell wall modification.

Drost et al. (2015) identified a major QTL peak on Chr10 for leaf width in an interspecific $P$. trichocarpa $\mathrm{x} P$. deltoides pseudobackcross family. This peak is in close proximity to one of our GWAS peaks for LA-LD-LL-LW. An ADP-ribosylation factor GTPase (PtARF1) was the prime candidate gene in the hybrid family based on eQTL analysis and functional assays (Drost et al., 2015). However, this gene was over 200kb from the closest associated SNP in our study (Potri.010G254700, a leucine-rich repeat transmembrane protein kinase). Furthermore, PtARF1 did not appear in our networks, indicating that it was not even weakly associated with leaf morphology in our population, and was not co-expressed or co-methylated with any weakly associated genes. This may indicate that different mechanisms control leaf morphology within P. trichocarpa compared to interspecific hybrids. However, the hypothesized mechanism for PtARF1 focuses on its role in vesicle-mediated trafficking of the PIN protein to regulate auxin gradients (Drost et al., 2015), which is broadly consistent with the genes in our network that affect cell wall extensibility and carbohydrate metabolism. Intriguingly, the co-expression network of Potri.001G173900 includes a gene (Potri.017G101100) for which the best homolog in Arabidopsis (AT3G02260) is a putative calossin-like protein required for polar auxin transport.

\subsubsection{Category 3}

This case study includes multitrait GWAS sets that had lower top SNP-trait association signals compared to the corresponding single trait GWAS for some loci. The multigrain GWAS set that included specific leaf area and leaf aspect ratio (SL-AR) well represents this category (Figure 2.5e). We detected four loci with suggestive associations for multitrait GWAS compared to 2 for single trait GWAS for specific leaf area (Figure 2.5e). In the case of locus Chr01_38557469, the association for SL was stronger than that for the AR-SL combination, and that locus explained $3.78 \%$ of the variation in SL (Supplementary Table S2.5). In contrast, there was no hint of an association for AR at that locus, possibly 
due to high variation in the minor allele homozygous individuals (Figure 2.5f). The loci for which the multitrait association showed the lowest $P$-value follow a similar pattern to those from Categories 1 and 2, with both traits showing differences among the genotypes (Supplementary Figure S2.10). For the ARSL multitrait we detected a total of 4 gene models, out of which one gene model overlapped with the single trait GWAS for specific leaf area (Figure 2.7). One of these, Potri.008G014000, belongs to the HSP-20 like chaperones superfamily. This gene is co-expressed with 49 other genes in P. trichocarpa, including 22 with putative roles in protein degradation or RNA processing, suggesting that this is another important regulatory network for leaf morphology (Supplementary Table S2.7).

\subsubsection{Comparison with previous GWAS studies in P. trichocarpa}

We compared all genes identified from single trait as well as the multitrait GWAS with the previous GWAS studies in P. trichocarpa using 34K Populus SNP array data (McKown et al., 2014b,c), but despite the moderate ( 0.2 to 0.4 ) heritabilities of most of the comparable traits such as leaf traits, height, chlorophyll content and stomatal density in these studies, none of the 22 genes we identified in our study overlapped with the previous studies. This might be due to the difference in the genotypes and the common garden used for our study, or to higher phenotyping error in the present study. It also likely reflects the more targeted genome sampling in the previous studies, which only assayed 3543 genes that were pre-selected based on annotations and other functional information (Geraldes et al., 2011). Nevertheless, we believe that the whole genome resequencing of 882 trees used in our study allowed the detection of robust genetic variants underlying some phenotypic traits. Some corroboration for these associations was provided by patterns of expression and co-expression, intersection with genetic control of metabolites, and direct confirmation of mutant phenotypes.

\subsection{Conclusion}

We have presented one of the most comprehensive GWAS studies to date for P. trichocarpa in terms of the size of the SNP dataset and the number of genotypes. Taking advantage of the natural variation present in the population and the power of multitrait association, we detected candidate genes that were associated with adaptive morphological and physiological traits. Some of these may represent genes with potentially pleiotropic effects on adaptive traits including leaf morphology, and water use efficiency. These have great potential for further functional characterization and can be a suitable target for breeding programs as they capture functional and structural relationships among the traits that are not apparent with single trait GWAS. Furthermore, the network analysis added an extra layer of information that provided further independent lines of evidence supporting the involvement of these genes in their associated phenotypes and provides clues about possible mechanisms of action. This is an important step in functional annotation, which remains a major challenge for recalcitrant model organisms like forest trees. 


\subsection{Tables and figures}

Table 2.1 Broad-sense heritability and the number of SNP-trait associations for morphological and physiological traits in P. trichocarpa.

\begin{tabular}{llllllll}
\hline Trait & $\begin{array}{l}H^{2} \\
(\mathrm{TPS})^{\mathrm{a}}\end{array}$ & $H^{2 \mathrm{~b}}$ & $\mathrm{~N}^{\mathrm{c}}$ & Total trees $^{\mathrm{d}}$ & $\begin{array}{l}\text { SNPs } \\
<1 \times 10^{-7 \mathrm{e}}\end{array}$ & PCs $^{\mathrm{f}}$ & ${\text { Chip_ } H^{2}(+/-\mathrm{CI})^{\mathrm{g}}}$ \\
\hline Morphology & & & & & & & \\
$\quad$ Height (HT) & 0.363 & 0.320 & 876 & $2378(851)$ & 0 & 27 & $1(+/-0.002)$ \\
$\quad$ Leaf area (LA) & 0.344 & 0.336 & 794 & $1056(262)$ & 0 & 23 & $0.793(+/-0.265)$ \\
Leaf aspect ratio (AR) & 0.462 & 0.477 & 794 & $1056(262)$ & 0 & 20 & $0.61(+/-0.312)$ \\
Leaf dry weight (LD) & 0.371 & 0.360 & 844 & $1094(250)$ & 0 & 26 & $0.751(+/-0.251)$ \\
Leaf length (LL) & 0.370 & 0.360 & 794 & $1056(262)$ & 0 & 22 & $0.766(+/-0.262)$ \\
Leaf perimeter (LP) & 0.362 & 0.351 & 794 & $1056(262)$ & 0 & 22 & $0.79(+/-0.262)$ \\
Leaf width (LW) & 0.344 & 0.346 & 794 & $1056(262)$ & 0 & 25 & $0.76(+/-0.266)$ \\
Petiole diameter (PD) & 0.297 & 0.184 & 839 & $1124(285)$ & 0 & 20 & $0.62(+/-0.263)$ \\
Petiole length (PL) & 0.561 & 0.562 & 839 & $1124(285)$ & 0 & 23 & $0.881(+/-0.28)$ \\
Specific leaf area (SL) & 0.371 & 0.376 & 784 & $1010(226)$ & 2 & 19 & $0.746(+/-0.257)$ \\
Stomatal density (SD) & 0.500 & 0.493 & 813 & $1064(251)$ & 1 & 16 & $0.834(+/-0.267)$ \\
Physiology & & & & & & & \\
Carbon isotope (CI) & 0.363 & 0.375 & 681 & $759(78)$ & 0 & 15 & $0.292(+/-0.337)$ \\
Leaf water potential (WP) & 0.080 & 0.000 & 823 & $964(141)$ & 0 & 15 & $0.322(+/-0.319)$ \\
SPAD 2014 (SP) & 0.310 & 0.297 & 839 & $1124(285)$ & 1 & 17 & $0.566(+/-0.331)$ \\
\hline
\end{tabular}

aBroad Sense Heritability with Thin Plate Spline correction (TPS) correction applied to the phenotypic data

${ }^{\mathrm{b} B r o a d}$ Sense Heritability without TPS correction.

'Number of genotypes.

${ }^{\mathrm{d} N u m b e r}$ of ramets sampled, with replicates in parentheses

${ }^{\text {e}} \mathrm{SNPs}$ with $P$-values $<1 \times 10^{-7}$ (suggestive significant SNPs)

${ }^{\mathrm{f}}$ Number of SNP PC covariates used in multitrait GWAS

${ }^{g}$ Mean Chip Heritability values for phenotypes with confidence interval (CI)

Note: All broad sense heritability estimates were significantly different from 0 except for WP. 
Table 2.2 List of traits for multitrait associations in P. trichocarpa.

\begin{tabular}{llrr}
\hline Trait Combination & Abbreviation & popN $^{\mathrm{a}}$ & PCs $^{\mathrm{b}}$ \\
\hline Carbon isotope, height, leaf area, petiole length & CI_HT_LA_PL & 632 & 14 \\
Carbon isotope, leaf area, stomatal density & CI_LA_SD & 603 & 12 \\
Carbon isotope, leaf area, SPAD, stomatal density & CI_LA_SD_SP & 600 & 12 \\
Carbon isotope, leaf water potential & CI_WP & 673 & 6 \\
Carbon isotope, leaf water potential, stomatal density & CI_SD_WP & 638 & 8 \\
Height, leaf area, petiole length & HT_LA_PL & 791 & 8 \\
Height, petiole diameter, petiole length & HT_PD_PL & 839 & 13 \\
Leaf area, leaf dry weight, leaf length, leaf width & LA_LD_LL_LW & 788 & 14 \\
Leaf area, SPAD, stomatal density & LA_SD_SP & 755 & 14 \\
Leaf aspect ratio, specific leaf area & AR_SL & 780 & 9 \\
Leaf dry weight, petiole diameter, SPAD & LD_PD_SP & 831 & 7 \\
Petiole diameter, petiole length, specific leaf area & PD_PL_SL & 781 & 17 \\
\hline
\end{tabular}

${ }^{a}$ Number of unique genotypes

${ }^{b}$ Number of SNP PC covariates used in multitrait GWAS

Table 2.3 Genes identified from P. trichocarpa single trait GWAS.

\begin{tabular}{llll}
\hline Gene model $^{\mathrm{a}}$ & Trait & $P$-value $^{\mathrm{b}}$ & Functional annotation $^{\mathrm{a}}$ \\
\hline Potri.001G371800 & Specific leaf area & $3.95 \mathrm{E}-08$ & NA $^{\mathrm{c}}$ \\
Potri.004G111000 & Specific leaf area & $9.76 \mathrm{E}-08$ & galacturonosyltransferase 9 \\
Potri.008G111800 & Stomatal density & $8.93 \mathrm{E}-08$ & 18S pre-ribosomal assembly protein \\
& & & gar2-related \\
Potri.010G098400 & SPAD & 4.84E-08 & Tetratricopeptide repeat (TPR)-like \\
& & & superfamily protein \\
\hline
\end{tabular}

\footnotetext{
${ }^{\mathrm{a}}$ Gene models are annotated using v3.1 of the $P$. trichocarpa genome

${ }^{\text {b }}$ SNP $P$-values $<1 \times 10^{-7}$

${ }^{\mathrm{c}} \mathrm{NA}$, Not available
} 
Table 2.4 Genes identified from P. trichocarpa multitrait GWAS.

\begin{tabular}{|c|c|c|c|}
\hline Gene model $^{\mathrm{a}}$ & Trait & $P$-value ${ }^{\mathrm{b}}$ & Functional annotation $^{\mathrm{a}}$ \\
\hline Potri.001G173900 & $\begin{array}{l}\text { Leaf area, leaf dry weight, } \\
\text { leaf length, leaf width }\end{array}$ & $1.61 \mathrm{E}-08$ & $\begin{array}{l}\text { Plant tudor-like RNA-binding } \\
\text { protein }\end{array}$ \\
\hline Potri.001G174300 & $\begin{array}{l}\text { Leaf area, leaf dry weight, } \\
\text { leaf length, leaf width }\end{array}$ & $9.15 \mathrm{E}-08$ & $\mathrm{NA}^{\mathrm{c}}$ \\
\hline Potri.001G189300 & $\begin{array}{l}\text { Leaf area, leaf dry weight, } \\
\text { leaf length, leaf width }\end{array}$ & $3.30 \mathrm{E}-08$ & NA \\
\hline Potri.001G371800 & $\begin{array}{l}\text { Leaf aspect ratio, specific } \\
\text { leaf area }\end{array}$ & 7.96E-08 & NA \\
\hline Potri.001G411800 & $\begin{array}{l}\text { Carbon isotope, leaf area, } \\
\text { stomatal density }\end{array}$ & 2.41E-08 & $\begin{array}{l}\text { EF-hand calcium-binding domain } \\
\text { containing protein }\end{array}$ \\
\hline Potri.002G055400 & $\begin{array}{l}\text { Leaf area, leaf dry weight, } \\
\text { leaf length, leaf width }\end{array}$ & $3.28 \mathrm{E}-08$ & Phytochrome interacting factor 4 \\
\hline Potri.002G145100 & $\begin{array}{l}\text { Carbon isotope, leaf area, } \\
\text { stomatal density }\end{array}$ & $2.62 \mathrm{E}-08$ & $\begin{array}{l}\text { Plant-specific transcription factor } \\
\text { YABBY family protein }\end{array}$ \\
\hline Potri.003G165400 & $\begin{array}{l}\text { Leaf aspect ratio, specific } \\
\text { leaf area }\end{array}$ & $6.62 \mathrm{E}-08$ & Gem-like protein 5 \\
\hline Potri.004G111000 & $\begin{array}{l}\text { Leaf area, leaf dry weight, } \\
\text { leaf length, leaf width }\end{array}$ & 4.72E-08 & Galacturonosyltransferase 9 \\
\hline Potri.004G153400 & $\begin{array}{l}\text { Leaf aspect ratio, specific } \\
\text { leaf area }\end{array}$ & $6.59 \mathrm{E}-08$ & $\begin{array}{l}\text { Similar to RAS-related GTP- } \\
\text { binding protein }\end{array}$ \\
\hline Potri.005G097900 & $\begin{array}{l}\text { Leaf area, SPAD, stomatal } \\
\text { density }\end{array}$ & 4.29E-08 & $\begin{array}{l}\text { Similar to oxidoreductase; } 2 \mathrm{OG}- \\
\mathrm{Fe}(2) \text { oxygenase family protein }\end{array}$ \\
\hline Potri.006G132500 & $\begin{array}{l}\text { Leaf area, leaf dry weight, } \\
\text { leaf length, leaf width }\end{array}$ & $1.57 \mathrm{E}-12$ & Ribosomal protein L4/L1 family \\
\hline Potri.006G134200 & $\begin{array}{l}\text { Carbon isotope, leaf area, } \\
\text { SPAD, stomatal density }\end{array}$ & 5.29E-08 & $\begin{array}{l}\text { Lysine-ketoglutarate } \\
\text { reductase/saccharopine } \\
\text { dehydrogenase bifunctional } \\
\text { enzyme }\end{array}$ \\
\hline Potri.008G121700 & $\begin{array}{l}\text { Carbon isotope, leaf water } \\
\text { potential, stomatal density }\end{array}$ & 5.43E-09 & NA \\
\hline
\end{tabular}




\begin{tabular}{|c|c|c|c|}
\hline Potri.008G144100 & $\begin{array}{l}\text { Leaf area, leaf dry weight, } \\
\text { leaf length, leaf width }\end{array}$ & 5.37E-08 & $\begin{array}{l}\text { Regulatory particle triple-A } \\
\text { ATPase 6A }\end{array}$ \\
\hline Potri.009G015500 & $\begin{array}{l}\text { Carbon isotope, leaf area, } \\
\text { stomatal density }\end{array}$ & $8.76 \mathrm{E}-09$ & $\begin{array}{l}\text { Mitochondrial transcription } \\
\text { termination factor family protein }\end{array}$ \\
\hline Potri.012G065600 & $\begin{array}{l}\text { Leaf area, petiole length, } \\
\text { height }\end{array}$ & $3.24 \mathrm{E}-08$ & Leo1-like family protein \\
\hline Potri.014G136400 & $\begin{array}{l}\text { Petiole diameter, petiole } \\
\text { length, specific leaf area }\end{array}$ & $2.66 \mathrm{E}-08$ & $\begin{array}{l}\text { LRR receptor-like } \\
\text { serine/threonine-protein kinase } \\
\text { RKF3-related }\end{array}$ \\
\hline Potri.016G071700 & $\begin{array}{l}\text { Carbon isotope, leaf area, } \\
\text { SPAD, stomatal density }\end{array}$ & $1.36 \mathrm{E}-08$ & NA \\
\hline \multirow[t]{2}{*}{ Potri.019G021600 } & $\begin{array}{l}\text { Leaf area, leaf dry weight, } \\
\text { leaf length, leaf width }\end{array}$ & 8.87E-08 & FtsH extracellular protease family \\
\hline & $\begin{array}{l}\text { Carbon isotope, height, leaf } \\
\text { area, petiole length }\end{array}$ & $1.56 \mathrm{E}-08$ & \\
\hline
\end{tabular}

${ }^{\mathrm{a}}$ Gene models are annotated using v3.1 of the P. trichocarpa genome.

${ }^{\text {b }}$ SNP $P$-values $<1 \times 10^{-7}$

${ }^{\mathrm{c}} \mathrm{NA}$, Not available 


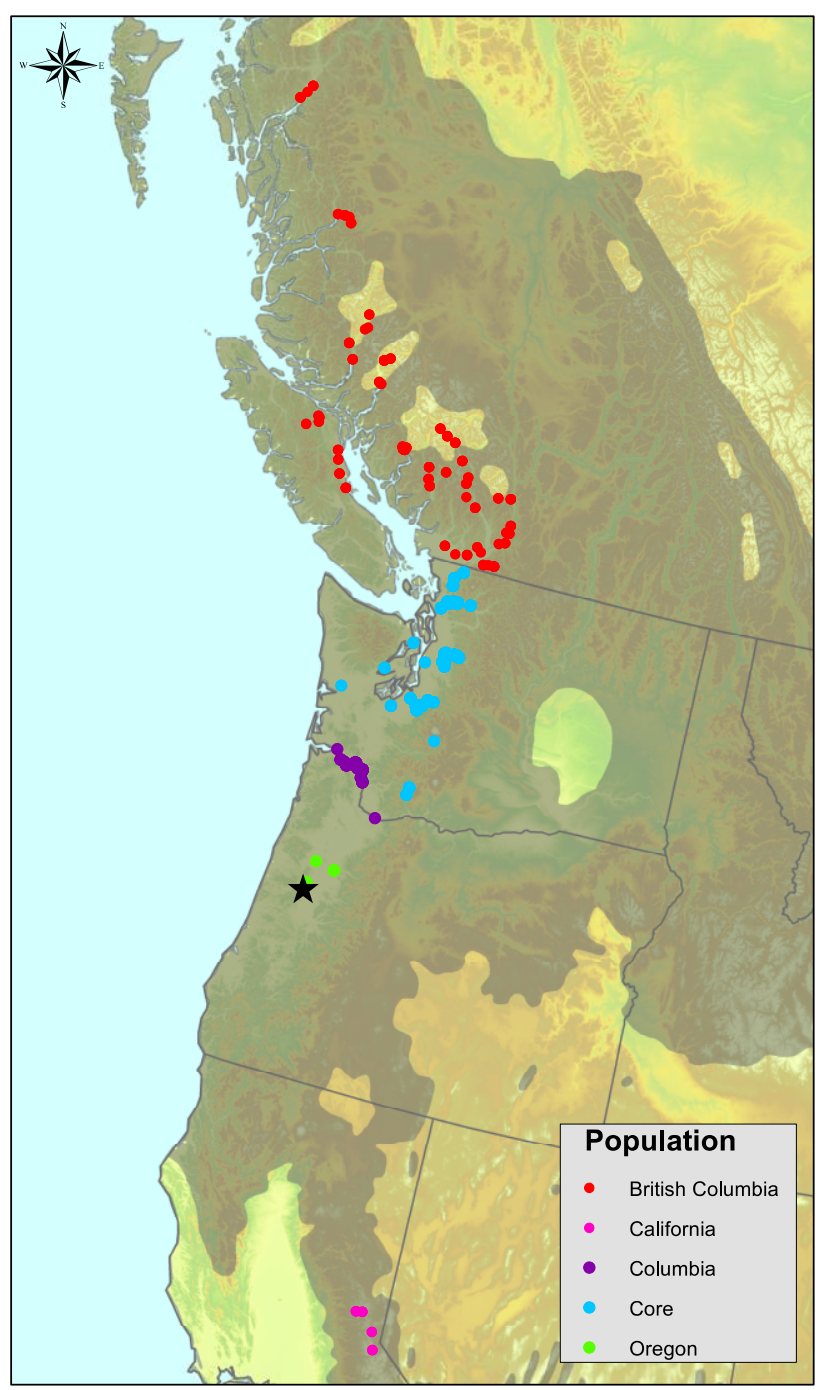

Figure 2.1 Source locations of 882 P. trichocarpa genotypes sampled in this study (colored dots). The trees were grown in a common garden in Corvallis, Oregon (black star). 


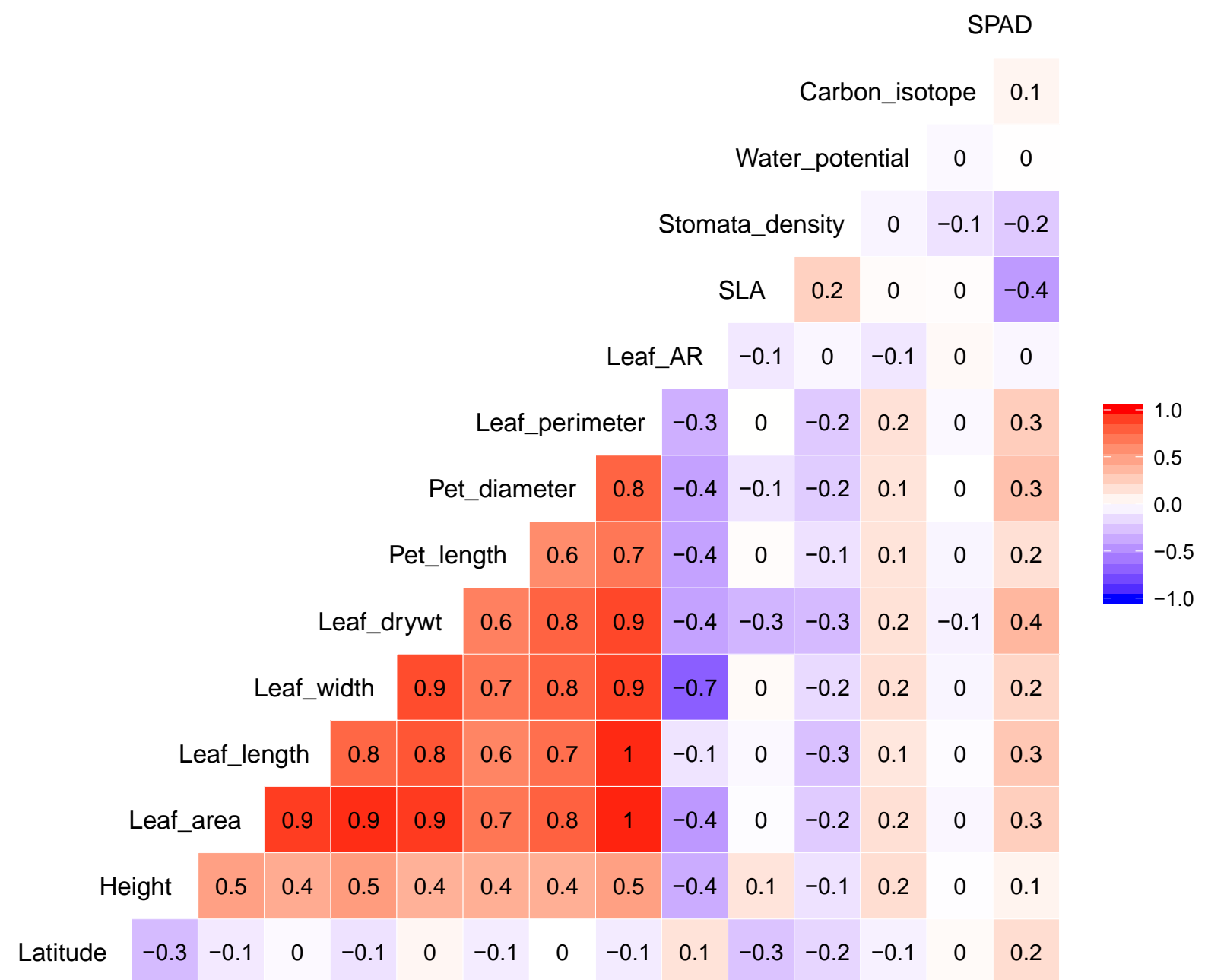

Figure 2.2 Pairwise Pearson genetic correlation of selected morphological and physiological traits (traits with at least 681 genotypes) measured in the P. trichocarpa common garden in Corvallis, Oregon. The color spectrum, bright red to bright blue represents highly positive to highly negative correlations and the number represents the correlation values. Best Linear Unbiased Predictor (BLUP) adjusted values were used. $P$-values are provided in Supplementary Table S2.2. SPAD represents leaf greenness. AR, aspect ratio; SLA, specific leaf area. 


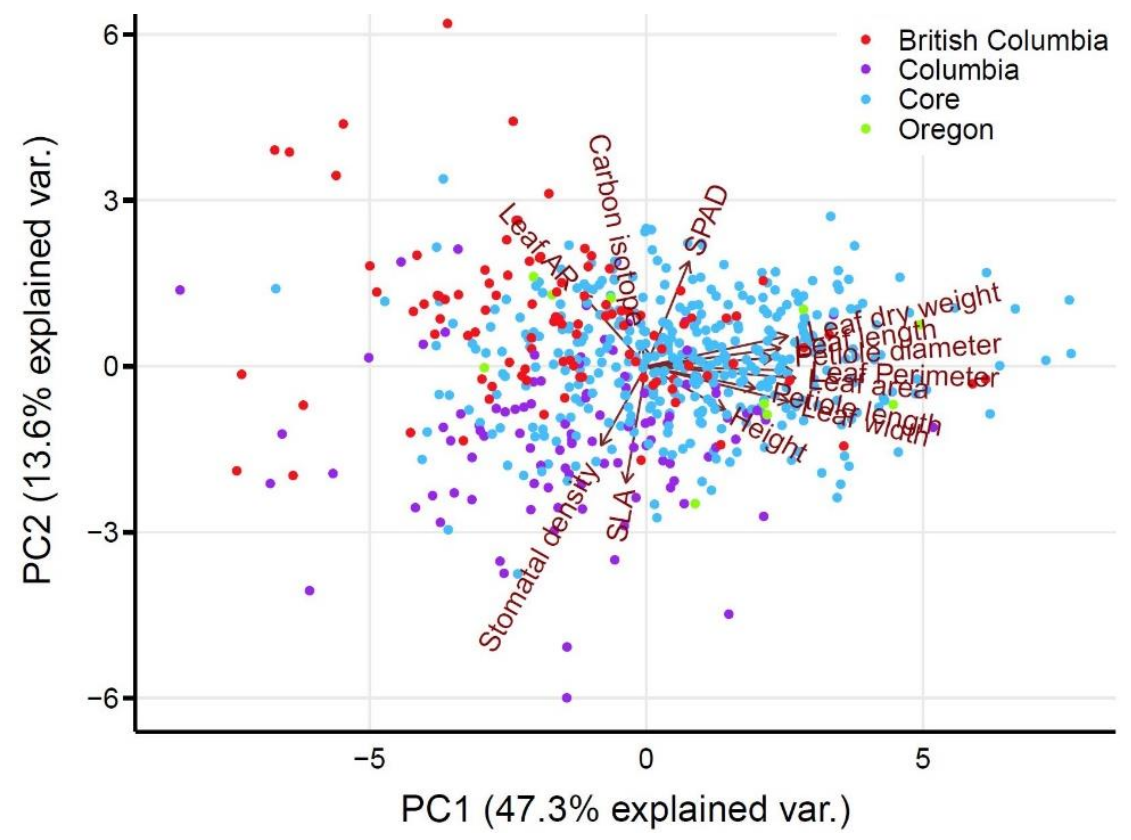

Figure 2.3 PCA biplot showing the first and second principal components with individual $P$.

trichocarpa genotypes (the points) colored by provenance as in Figure 2.1, and relative weightings of the explanatory variables indicated by vectors. BC, British Columbia; OR, Oregon.

(a)

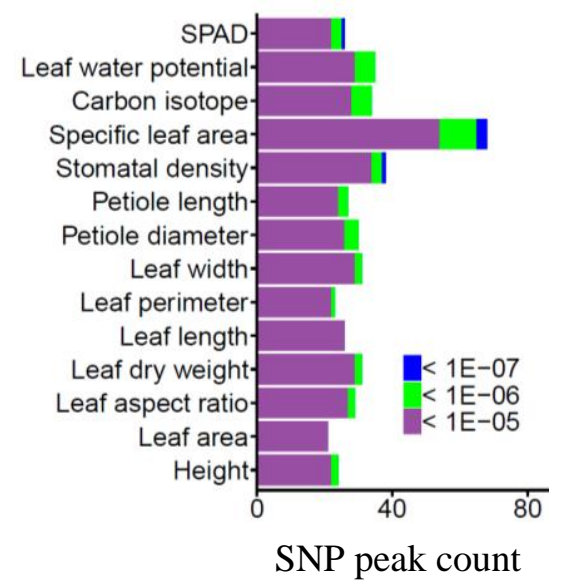

(b)

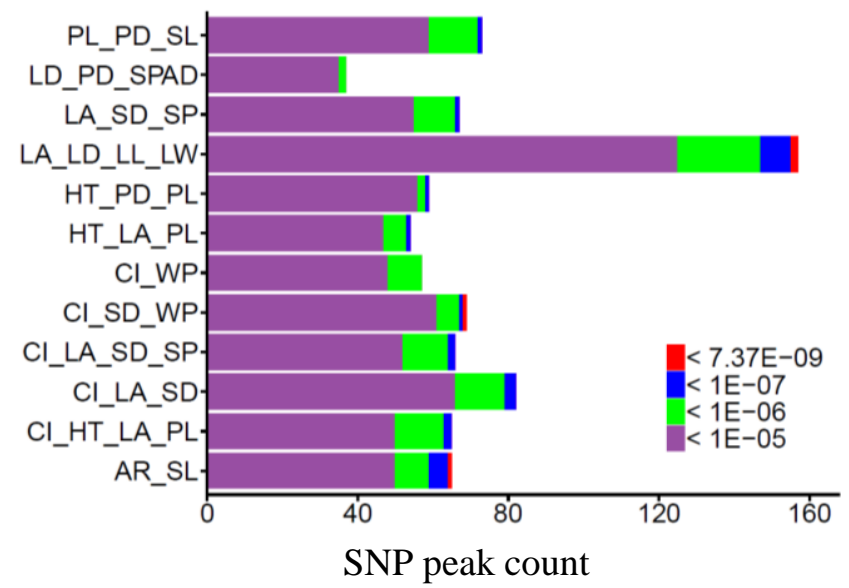

Figure 2.4 P. trichocarpa SNP-trait association peak counts at 10kb intervals. (a) Single trait GWAS (b) Multitrait GWAS. 
(a)

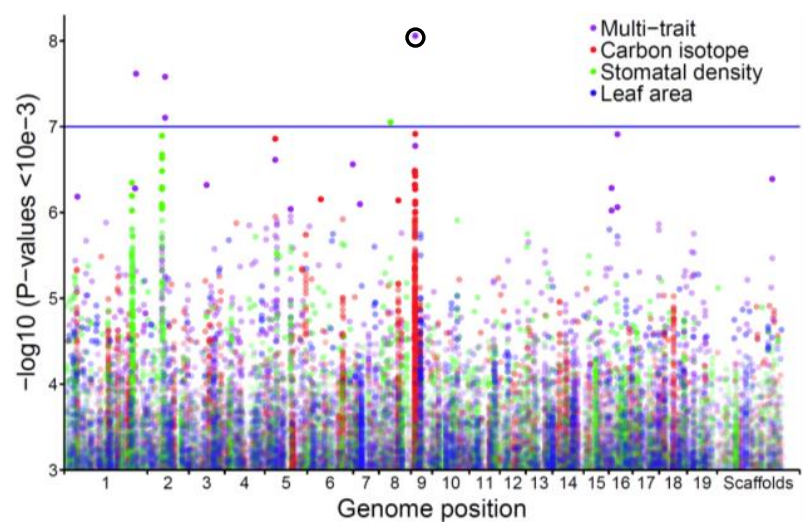

(c)

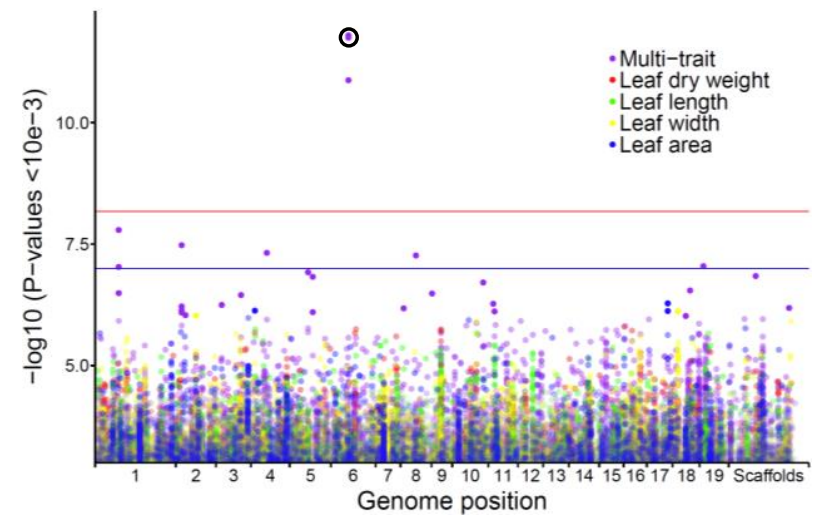

(e)

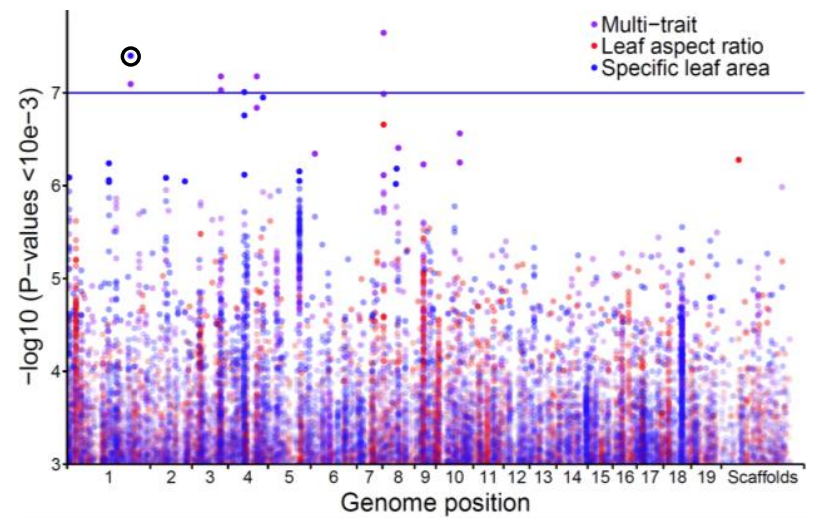

(b)

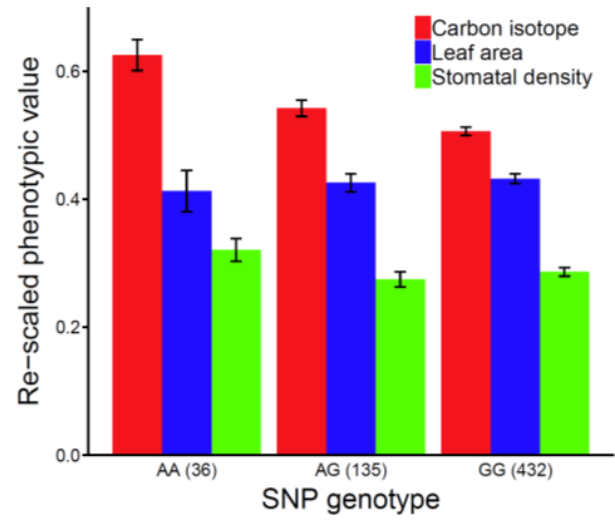

(d)

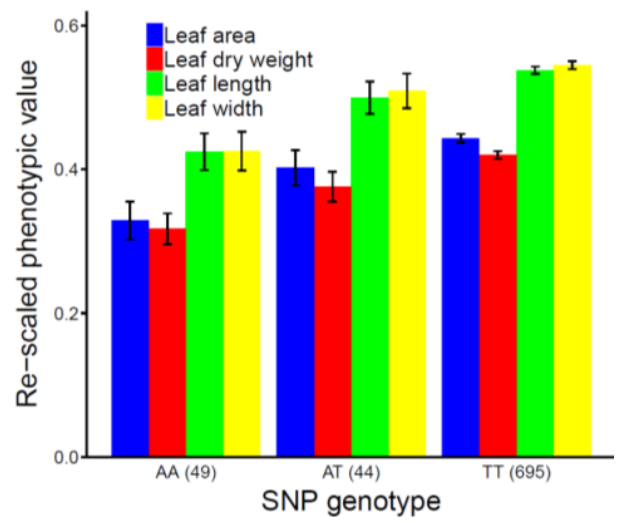

(f)

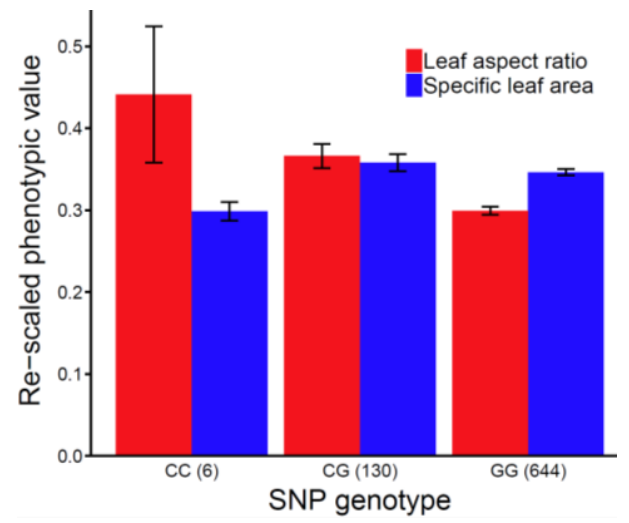

Figure 2.5 Manhattan plots comparing GEMMA univariate and multivariate GWAS in P. trichocarpa. The colors of the dots correspond to single trait or multitrait associations. $P$-values are converted to $\log 10$ ( $P$-value). SNPs above red lines passed Bonferroni correction test $\left(P<7.37 \times 10^{-9}\right)$, SNPs above blue lines are considered suggestive associations $\left(P<1 \times 10^{-7}\right)$. Only SNPs with $P<1 \times 10^{-3}$ are plotted. (a) Carbon isotope, stomatal density, and leaf area. (b) Allelic effects of SNP near Potri.009G015500. (c) Leaf area, 
leaf dry weight, leaf length, and leaf width. (d) Allelic effects of SNP near gene Potri.006G132500. (e) Leaf aspect ratio and specific leaf area. (f) Allelic effects of SNP near gene Potri.001G371800. SNPs depicted in Figures b, d, and $\mathrm{f}$ are circled in the corresponding Manhattan plots and the error bars in b, $\mathrm{d}$, and $\mathrm{f}$ represent \pm standard error for re-scaled phenotypic values.

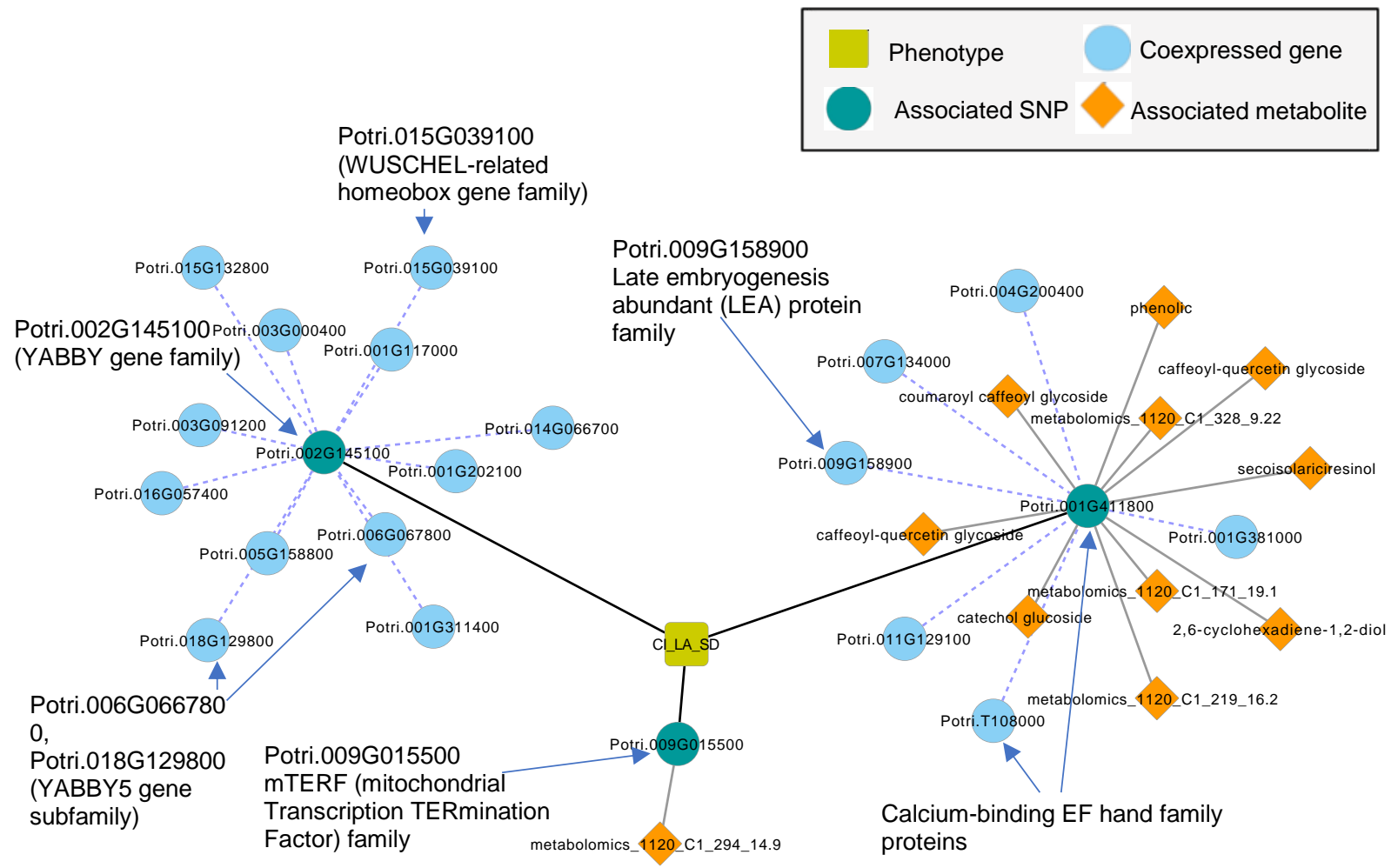

Figure 2.6 Merged network for carbon isotope, leaf area and stomatal density (CI_LA_SD) in $P$.

trichocarpa. Networks of co-expressed genes were based on RNAseq data for 14 tissue types from the Phytozome Populus gene atlas. Networks of associated metabolites were based on GWAS for the same population that was used here (Weighill et al., 2018). 


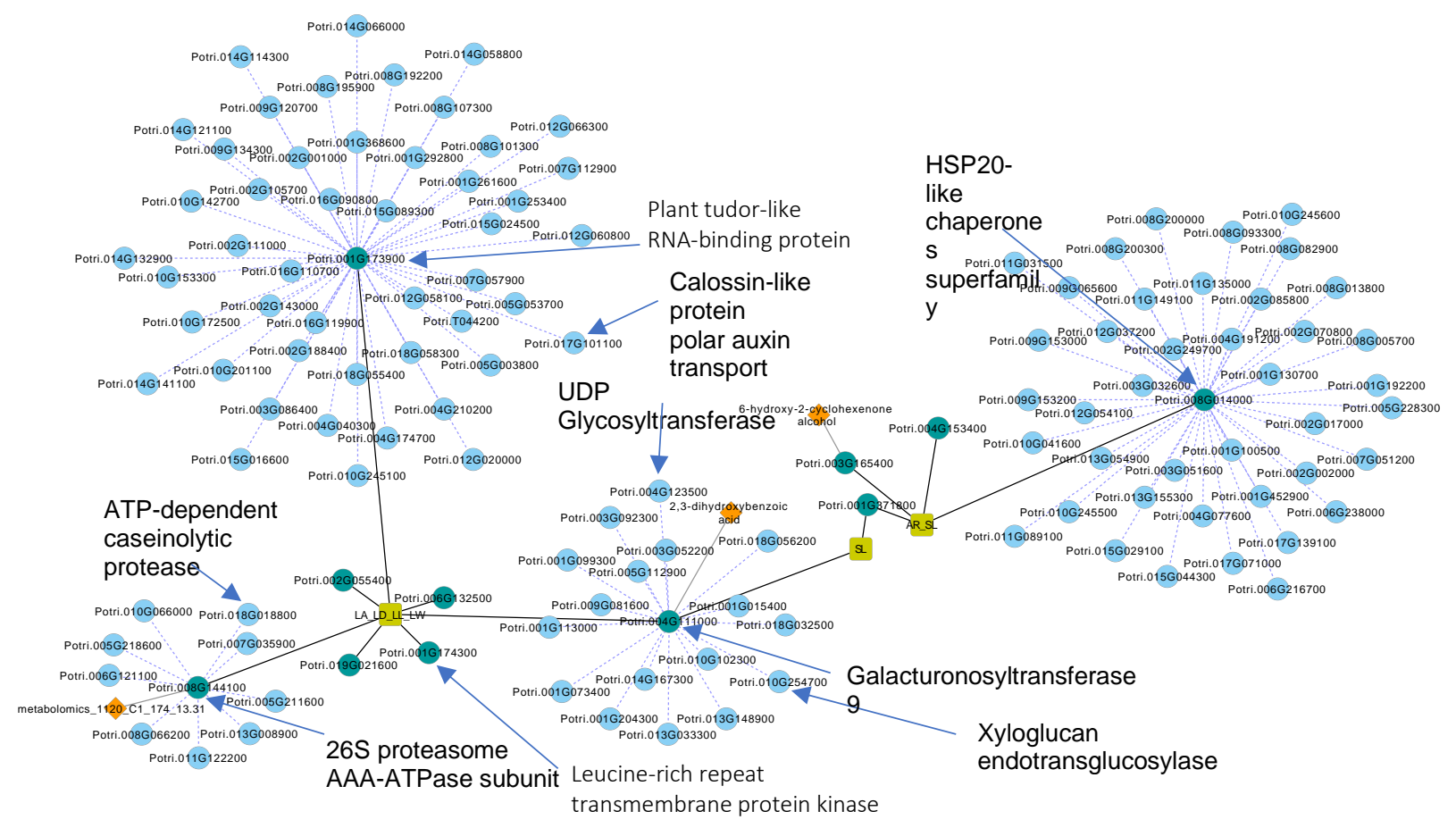

Figure 2.7 Merged network for leaf area, leaf dry weight, leaf length, and leaf width (LA_LD_LL_LW) and leaf aspect ratio-specific leaf area (AR_SL) in P. trichocarpa. Symbols are as defined in Figure 2.6. 
(a)

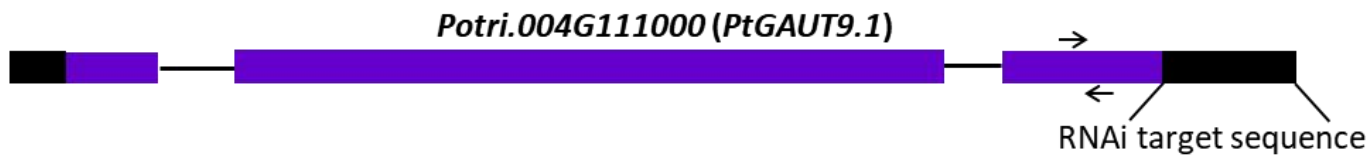

(b)

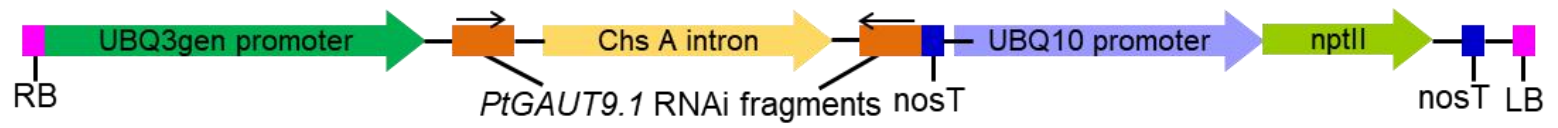

(c)

(d)

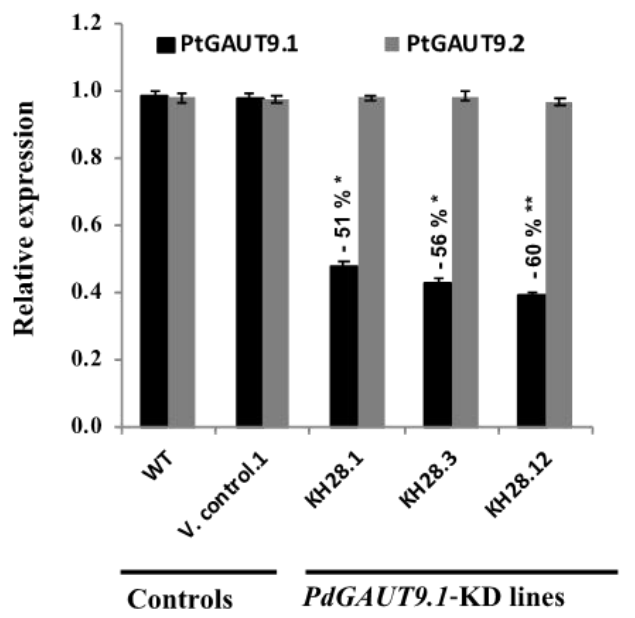

(e)

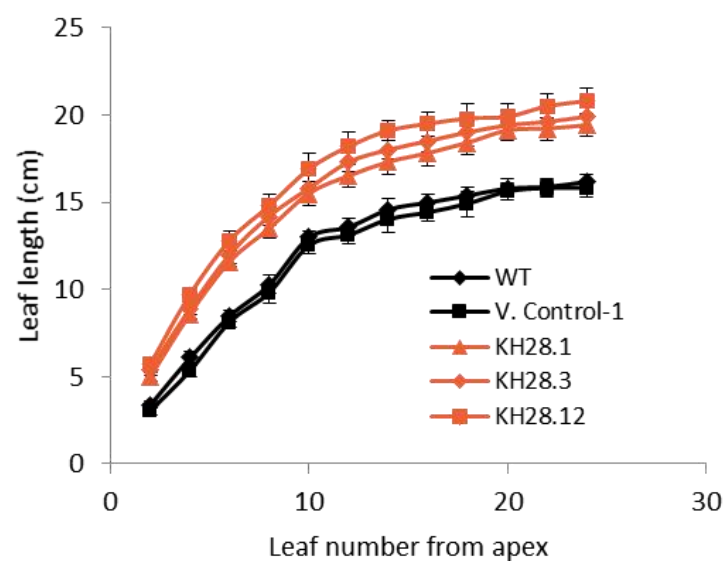

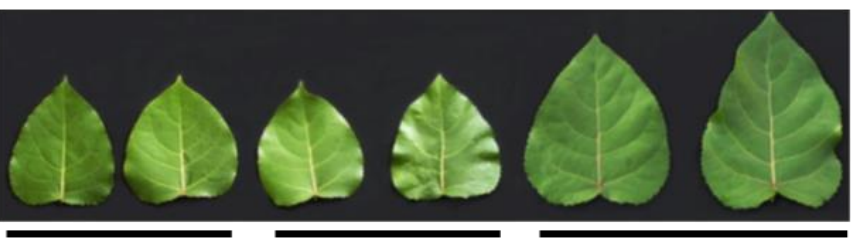

WT $\mathrm{VC}$ PdGAUT9.1-KD lines (f)

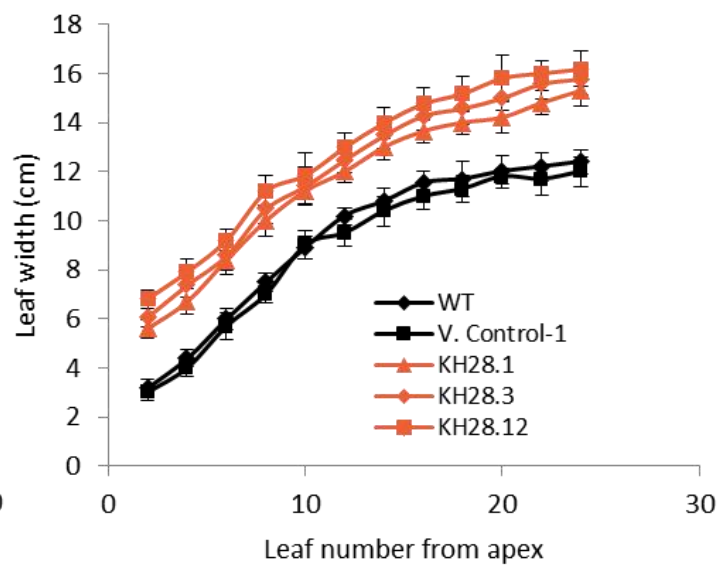

Figure 2.8 Effects of PdGAUT9.1 down-regulation on leaf size in P. deltoides. (a) Gene model for PtGAUT9.1 (Potri.004G111000) from Populus trichocarpa v3.0 genome. Black boxes indicate the 5' and 3' untranslated regions (UTRs); purple boxes indicate exons and lines indicate introns. The indicated RNAi targeted sequence was $123 \mathrm{bp}$. The sequences used for quantitative RT-PCR are indicated by arrows. (b) Schematic representation of PtGAUT9.1 RNAi silencing construct used to generate $P$. deltoides PdGAUT9.1-KD transgenic lines. (c) Relative transcript abundance of PdGAUT9.1 (Potri.004G111000) and PdGAUT9.2 (Potri.017G106800) as determined by quantitative RT-PCR 
analysis of leaf RNA from greenhouse-grown 3-month-old poplar WT, vector control (V. control.1) and PdGAUT9.1-KD lines (KH28.1, KH28.3 and KH28.12). Expression of PdGAUT9.1 in poplar WT was set to 1 and $18 S$ rRNA was used as a reference gene. Error bars are SE, $\mathrm{n}=6$. Differences were tested by oneway ANOVA $(P<0.05 ; P<0.001)$. (d) Leaf phenotype (the sixth leaf from the apex) of $P$. deltoides control (WT and VC) and PdGAUT9.1-KD line (KH28.12) from 3-month-old plants. (e) Length and (f) width of leaves from different developmental stages of three different 3-month-old GAUT9-KD transgenic lines (KH28.1, KH28.3 and KH28.12). Every other leaf of ten plants was measured starting with the $2^{\text {nd }}$ leaf from the apex. The error bars represent \pm standard error for leaf length and leaf width. 


\subsection{Supplementary tables and figures}

Supplementary Table S 2.1 SNP PC covariates used in P. trichocarpa single and multitrait GWAS analyses.

\begin{tabular}{|c|c|c|c|}
\hline & Trait & SNP PC covariates* & $\begin{array}{l}\text { SNP PC } \\
\text { count }\end{array}$ \\
\hline \multirow{14}{*}{ Single-trait } & Height (HT) & $1,3-7,10,12-13,16-18,20-22,26-28,31,35-36,38,42,44,49,51,57$ & 27 \\
\hline & Leaf area (LA) & $1,3-7,10-13,16,20,22,28,32,38,44-45,47,49,50,57,60$ & 23 \\
\hline & Leaf aspect ratio (AR) & $2-7,9,13,21-22,34-35,41-43,47,49-50,57-58$ & 20 \\
\hline & Leaf dry weight (LD) & $1,3,4,6-7,9,10-13,16,19-22,32,36,38,42-45,47,49,51,57$ & 26 \\
\hline & Leaf length (LL) & $1,3-7,10,12-13,15-17,20,22,28,32,38,44,46-47,51,60$ & 22 \\
\hline & Leaf perimeter (LP) & $1,3-7,10,12,13,17,20,22,25,28,32,38,44,46-47,49,57,60$ & 22 \\
\hline & Leaf width (LW) & $1-4,6-7,9-13,16,20-22,28,32,38,41,43,47,49,50,57,60$ & 25 \\
\hline & Petiole diameter (PD) & $1-4,6-7,11-13,18,22,28,32,36,43,46-47,51,57,60$ & 20 \\
\hline & Petiole length (PL) & $1-7,11-13,15-17,22,27,32,40,46-47,50-51,54,57$ & 23 \\
\hline & Specific leaf area (SL) & $1-3,5,7,10-11,13,16,19,24,36,40-43,50,55,59$ & 19 \\
\hline & Stomatal density (SD) & $1-2,5-6,13,18,22,24,34,39,46,48,50,52,57-58$ & 16 \\
\hline & Carbon isotope (CI) & $2-3,6-8,15-16,21-22,28,31,47,48,53,59$ & 15 \\
\hline & Leaf water potential (WP) & $1,3-4,7,9,12,17,20,22,28,33-34,47,49,52$ & 15 \\
\hline & $\mathrm{SPAD}_{2014}(\mathrm{SP})$ & $1,4,6-7,15,19,21,24,29-30,32,40,42-43,52,54,60$ & 17 \\
\hline \multirow{12}{*}{ Multi-trait } & Carbon isotope, height, leaf area, petiole length (CI_HT_LA_PL) & $1-7,12-13,22,25,38,47,57$ & 14 \\
\hline & Carbon isotope, leaf area, stomatal density (CI_LA_SD) & $3-5,7,12-13,18,25,38,39,47,57$ & 12 \\
\hline & Carbon isotope, leaf area, SPAD, stomatal density (CI_LA_SD_SP) & $3-5,7,12-13,18,25,38,39,47,57$ & 12 \\
\hline & Carbon isotope, leaf water potential (CI_WP) & $1-2,7,8,15,28$ & 6 \\
\hline & Carbon isotope, leaf water potential, stomatal density (CI_SD_WP) & $1-3,4,6,18,22,57$ & 8 \\
\hline & Height, leaf area, petiole length (HT_LA_PL) & $1,3-6,18,22,57$ & 8 \\
\hline & Height, petiole diameter, petiole length (HT_PD_PL) & $1,3-6,7,11-13,16,22,32,47$ & 13 \\
\hline & Leaf area, leaf dry weight, leaf length, leaf width (LA_LD_LL_LW) & $1,3-4,6-7,12-13,22,32,38,44,47,49,57$ & 14 \\
\hline & Leaf area, SPAD, stomatal density (LA_SD_SP) & $3-5,7,12-13,18,32,38,47,49-50,57,60$ & 14 \\
\hline & Leaf aspect ratio, specific leaf area (AR_SL) & $1,3,5,7,10-11,36,40,59$ & 9 \\
\hline & Leaf dry weight, petiole diameter, SPAD (LD_PD_SP) & $1,6-7,29,32,43,60$ & 7 \\
\hline & Petiole diameter, petiole length, specific leaf area (PD_PL_SL) & $1-7,10,13,17,22,27,32,36,42,47,50$ & 17 \\
\hline
\end{tabular}

* PC covariates were selected based on the stepwise regression using both forward and backward selection methods 
Supplementary Table S 2.2 Pearson pairwise correlation of morphological and physiological traits collected in $P$. trichocarpa association population; the numbers below diagonal represent correlation values; the numbers above diagonal represent $P$-values. Red and blue colors indicate positive and negative correlations or $P$-values, respectively.

\begin{tabular}{|c|c|c|c|c|c|c|c|c|c|c|c|c|c|c|c|c|c|}
\hline \multirow[b]{2}{*}{ 总 } & \multirow[b]{2}{*}{ 畄 } & \multicolumn{9}{|c|}{ Morphology } & \multicolumn{5}{|c|}{ Physiology } & \multirow[b]{2}{*}{ 善 } & \multirow[b]{2}{*}{ 产 } \\
\hline & & 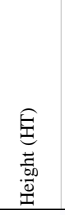 & 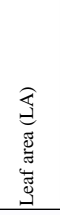 & 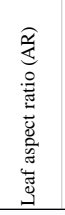 & 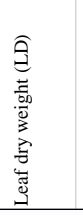 & 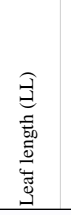 & 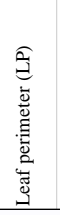 & 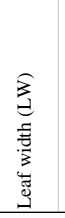 & 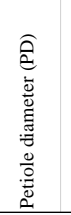 & 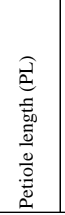 & 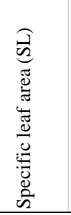 & 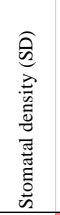 & 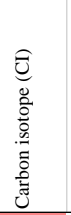 & 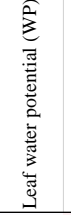 & $\begin{array}{l}\hat{\hat{n}} \\
\hat{2} \\
\hat{z} \\
\hat{n}\end{array}$ & & \\
\hline \multirow{11}{*}{ 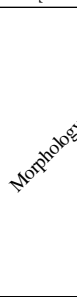 } & Height (HT) & NA & 0 & 0 & 0 & 0 & 0 & 0 & 0 & 0 & 0 & 0 & 0.782 & 0 & 0.079 & \begin{tabular}{|l|l|}
9 & Height (HT) \\
\end{tabular} & \\
\hline & Leaf area (LA) & 0.489 & & 0 & 0 & 0 & 0 & 0 & 0 & 0 & 0.681 & 0 & 0.285 & 0 & & Leaf area (LA) & \\
\hline & Leaf aspect ratio (AR) & -0.356 & -0.443 & NA & 0 & 0.001 & 0 & 0 & 0 & 0 & 0.004 & 0.252 & 0.368 & 0.002 & 0.274 & Leaf aspect ratio (AR) & \\
\hline & Leaf dry weight (LD) & 0.407 & 0.91 & -0.399 & NA & 0 & 0 & 0 & 0 & 0 & 0 & 0 & 0.087 & 0 & & \multirow{5}{*}{$\begin{array}{ll}0 & \text { Leaf dry weight (LD) } \\
0 & \text { Leaf length (LL) } \\
0 & \text { Leaf perimeter (LP) } \\
0 & \text { Leaf width (LW) } \\
0 & \text { Petiole diameter (PD) }\end{array}$} & \\
\hline & Leaf length (LL) & 0.437 & 0.904 & -0.114 & 0.821 & NA & 0 & 0 & 0 & 0 & 0.399 & 0 & 0.606 & 0 & & & \\
\hline & Leaf perimeter (LP) & 0.493 & 0.963 & -0.342 & $0.871^{\circ}-\operatorname{lo}$ & 0.952 & $\mathrm{NA}$ & 0 & 0 & 0 & 0.942 & 0 & 0.41 & 0 & & & \\
\hline & Leaf width (LW) & 0.513 & 0.944 & -0.695 & 0.857 & 0.754 & 0.883 & NA & 0 & 0 & 0.455 & 0 & 0.215 & 0 & & & \\
\hline & Petiole diameter (PD) & 0.417 & 0.778 & -0.402 & 0.769 & 0.719 & 0.764 & 0.764 & NA & 0 & 0.001 & 0 & 0.963 & 0 & & & \\
\hline & Petiole length (PL) & 0.417 & 0.691 & -0.401 & 0.642 & 0.623 & 0.684 & 0.689 & 0.589 & $\mathrm{NA}$ & 0.623 & 0 & 0.219 & 0 & & \multirow{2}{*}{\begin{tabular}{l|l}
0 & Petiole length $(\mathrm{PL})$ \\
0 & Specific leaf area (SL)
\end{tabular}} & \\
\hline & Specific leaf area (SL) & 0.14 & -0.014 & -0.098 & -0.318 & -0.029 & 0.002 & 0.025 & -0.116 & 0.017 & NA & 0 & 0.695 & 0.564 & & & \\
\hline & Stomatal density (SD) & -0.124 & -0.22 & -0.039 & -0.293 & -0.271 & -0.231 & -0.159 & -0.219 & -0.128 & 0.241 & NA & 0 & 0.176 & & Stomatal density (SD) & \\
\hline \multirow[b]{3}{*}{20} & Carbon isotope (CI) & -0.009 & -0.036 & 0.03 & -0.058 & -0.017 & -0.028 & -0.042 & 0.002 & -0.042 & 0.013 & -0.12 & $\mathrm{NA}$ & 0.377 & 0.116 & \multirow{2}{*}{\begin{tabular}{l|l|}
6 & Carbon isotope (CI) \\
1 & Leaf water potential (WP)
\end{tabular}} & \multirow{3}{*}{$209^{90}$} \\
\hline & Leaf water potential (WP) & 0.175 & 0.164 & -0.103 & 0.153 & 0.128 & 0.155 & 0.161 & 0.141 & 0.142 & 0.02 & -0.05 & -0.03 & NA & 0.931 & & \\
\hline & $\operatorname{SPAD}(\mathrm{SP})$ & 0.059 & 0.252 & -0.037 & 0.378 & 0.291 & 0.262 & 0.217 & 0.322 & 0.163 & -0.436 & -0.23 & 0.053 & 0.003 & & $\operatorname{SPAD}_{2014}(\mathrm{SP})$ & \\
\hline
\end{tabular}


Supplementary Table S 2.3 PCA loadings of the traits of 13 morphological and physiological traits used in PCAbiplot (Figure 2.3) collected in P. trichocarpa association plantation in Corvallis, OR. Red and blue colors indicate positive and negative loadings, respectively. The first five PCs explain more than $83 \%$ of the variation in the traits. PC1, PC2, PC3, PC4 and PC5 explain 47\%, 14\%, 9\%, $8 \%$ and $6 \%$ of the total variation, respectively.

\begin{tabular}{|lrr|rrr|}
\hline Traits & PC1 & \multicolumn{1}{l}{ PC2 } & PC3 & PC4 & \multicolumn{1}{l}{ PC5 } \\
\hline Carbon isotope & -0.011 & 0.103 & 0.512 & -0.737 & 0.390 \\
\hline Height & 0.207 & -0.213 & 0.211 & -0.123 & -0.536 \\
\hline Leaf AR & -0.171 & 0.358 & 0.471 & 0.516 & 0.184 \\
\hline Leaf area & 0.392 & -0.058 & 0.036 & 0.064 & 0.093 \\
\hline Leaf dry weight & 0.372 & 0.148 & -0.126 & 0.010 & 0.031 \\
\hline Leaf length & 0.353 & 0.088 & 0.275 & 0.293 & 0.167 \\
\hline Leaf Perimeter & 0.383 & -0.021 & 0.139 & 0.153 & 0.123 \\
\hline Leaf width & 0.375 & -0.174 & -0.138 & -0.133 & 0.005 \\
\hline Petiole diameter & 0.335 & 0.041 & -0.027 & -0.035 & 0.088 \\
\hline Petiole length & 0.287 & -0.108 & -0.033 & 0.033 & 0.085 \\
\hline SLA & -0.053 & -0.571 & 0.392 & 0.111 & 0.114 \\
\hline SPAD & 0.113 & 0.513 & -0.233 & -0.138 & 0.175 \\
\hline Stomatal density & -0.118 & -0.387 & -0.362 & 0.101 & 0.645 \\
\hline
\end{tabular}


Supplementary Table S 2.4 Pearson correlation of morphological and physiological traits with latitude of origin in P. trichocarpa.

\begin{tabular}{|c|c|c|}
\hline Traits & $\begin{array}{l}\text { Correlation with } \\
\text { latitude (r) }\end{array}$ & $P$-value \\
\hline Height (HT) & -0.292 & $<0.001^{*}$ \\
\hline Petiole length (PL) & -0.082 & $0.017 *$ \\
\hline Petiole diameter (PD) & -0.001 & 0.976 \\
\hline Leaf area (LA) & -0.092 & $0.009 *$ \\
\hline Leaf perimeter (LP) & -0.086 & $0.016^{*}$ \\
\hline Leaf length (LL) & -0.026 & 0.458 \\
\hline Leaf width (LW) & -0.125 & $<0.001 *$ \\
\hline Leaf aspect ratio (AR) & 0.147 & $<0.001 *$ \\
\hline Leaf dry weight (LD) & 0.047 & 0.175 \\
\hline Specific leaf area (SL) & -0.256 & $<0.001 *$ \\
\hline SPAD (SP) & 0.176 & $<0.001 *$ \\
\hline Stomatal density (SD) & -0.175 & $<0.001 *$ \\
\hline Leaf water potential (WP) & -0.058 & 0.096 \\
\hline Carbon isotope $(\mathrm{CI})$ & 0.026 & 0.504 \\
\hline $\begin{array}{l}\mathrm{PC} 1 \text { of all traits except leaf } \\
\text { feret and leaf water potential }\end{array}$ & -0.171 & $<0.001 *$ \\
\hline $\begin{array}{l}\text { PC2 of all traits except leaf } \\
\text { feret and leaf water potential }\end{array}$ & 0.409 & $<0.001 *$ \\
\hline$*$ Significant $P$-values & & \\
\hline
\end{tabular}

Note: Supplementary Tables S2.5, S2.6 and S2.7 are too large to fit here, but are available online at this link: https://nph.onlinelibrary.wiley.com/doi/10.1111/nph.15777. 
(a)

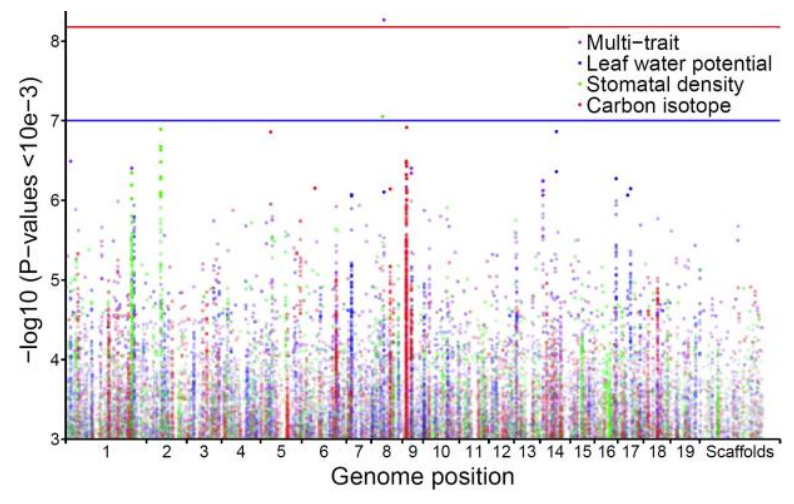

(b)

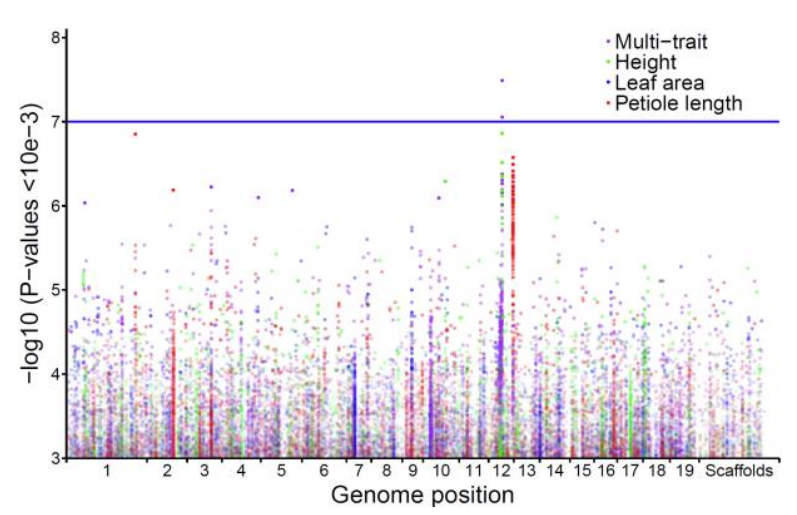

(c)

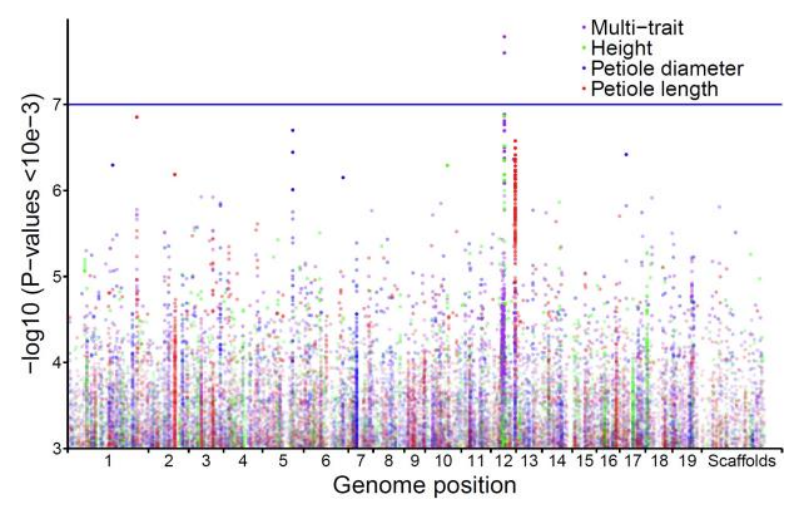

Supplementary Figure S 2.1 Manhattan plots comparing GEMMA univariate and multivariate GWAS in $P$. trichocarpa. The colors of the dots correspond to single trait or multitrait (purple) associations. $P$ values are converted to $-\log 10$ ( $P$-value). SNPs above red lines passed Bonferroni correction test $\left(P<7.37 \times 10^{-9}\right)$, SNPs above blue lines are considered suggestive associations $\left(P<1 \times 10^{-7}\right)$. Only SNPs with $P<1 \times 10^{-3}$ are plotted. (a) Stomatal density (green), carbon isotope (red), and pre-dawn leaf water potential (blue). (b) Height (green), leaf area (blue), and petiole length (red). (c) Height (green), petiole diameter (blue), and petiole length (red). 
(a)

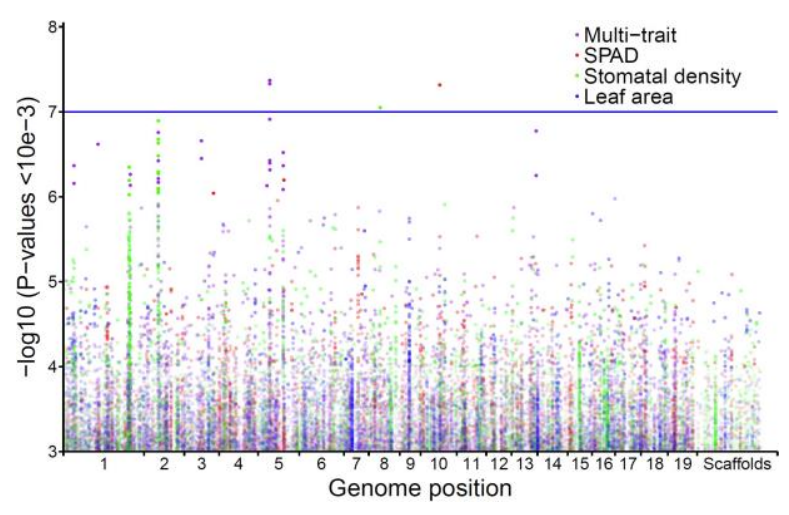

(c) (b)

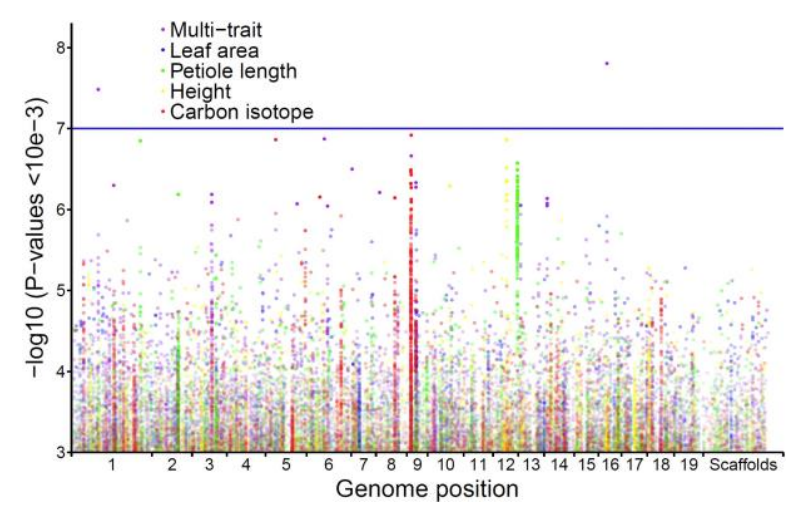

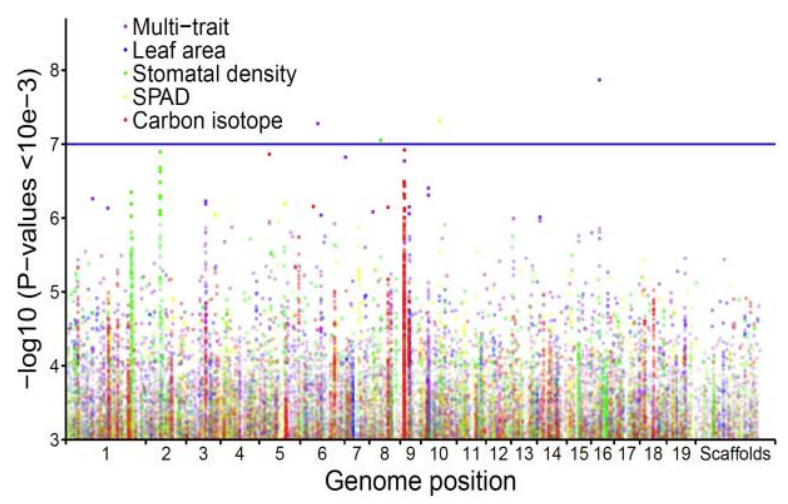

Supplementary Figure S 2.2 Manhattan plots comparing GEMMA univariate and multivariate GWAS in $P$. trichocarpa. The colors of the dots correspond to single trait or multitrait (purple) associations. $P$ values are converted to $-\log 10$ ( $P$-value). SNPs above blue lines are considered suggestive associations $\left(P<1 \times 10^{-7}\right)$. Only SNPs with $P<1 \times 10^{-3}$ are plotted. (a) Leaf area (blue), stomatal density (green), and SPAD (red). (b) Leaf area (blue), petiole length (green), height (yellow), and carbon isotope (red). (c) Leaf area (blue), stomatal density (green), SPAD (yellow), and carbon isotope (red). 
(a)

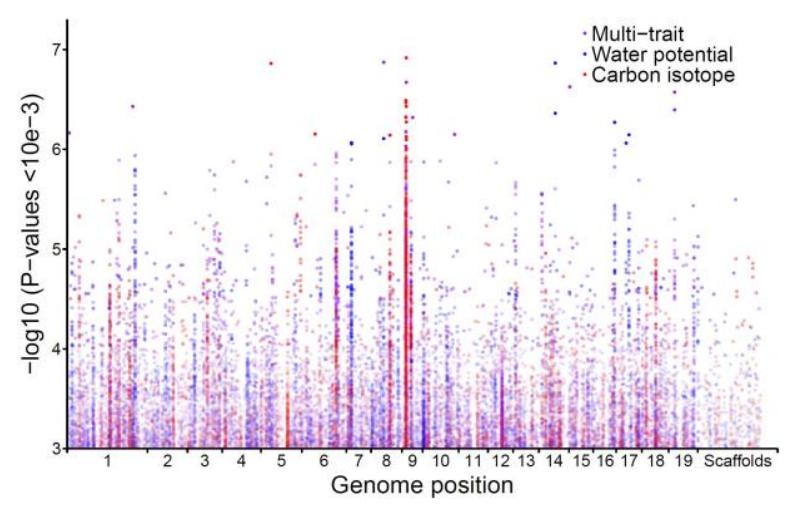

(b)

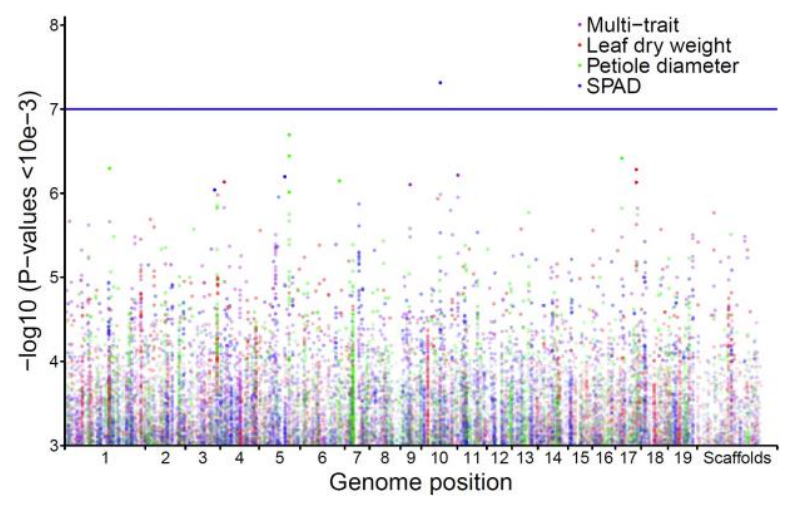

(c)

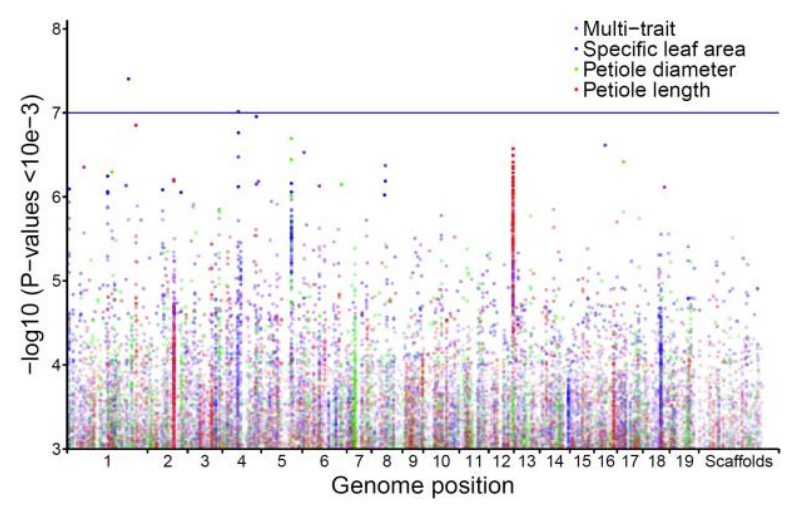

Supplementary Figure S 2.3 Manhattan plots comparing GEMMA univariate and multivariate GWAS in $P$. trichocarpa. The colors of the dots correspond to single trait or multitrait (purple) associations. $P$ values are converted to $-\log 10$ ( $P$-value). SNPs above blue lines are considered suggestive associations $\left(P<1 \times 10^{-7}\right)$. Only SNPs with $P<1 \times 10^{-3}$ are plotted. (a) Carbon isotope (red), pre-dawn leaf water potential (blue). (b) Leaf dry weight (red), petiole diameter (green), SPAD (blue). (c) Petiole diameter (green), petiole length (red), SLA (blue). 

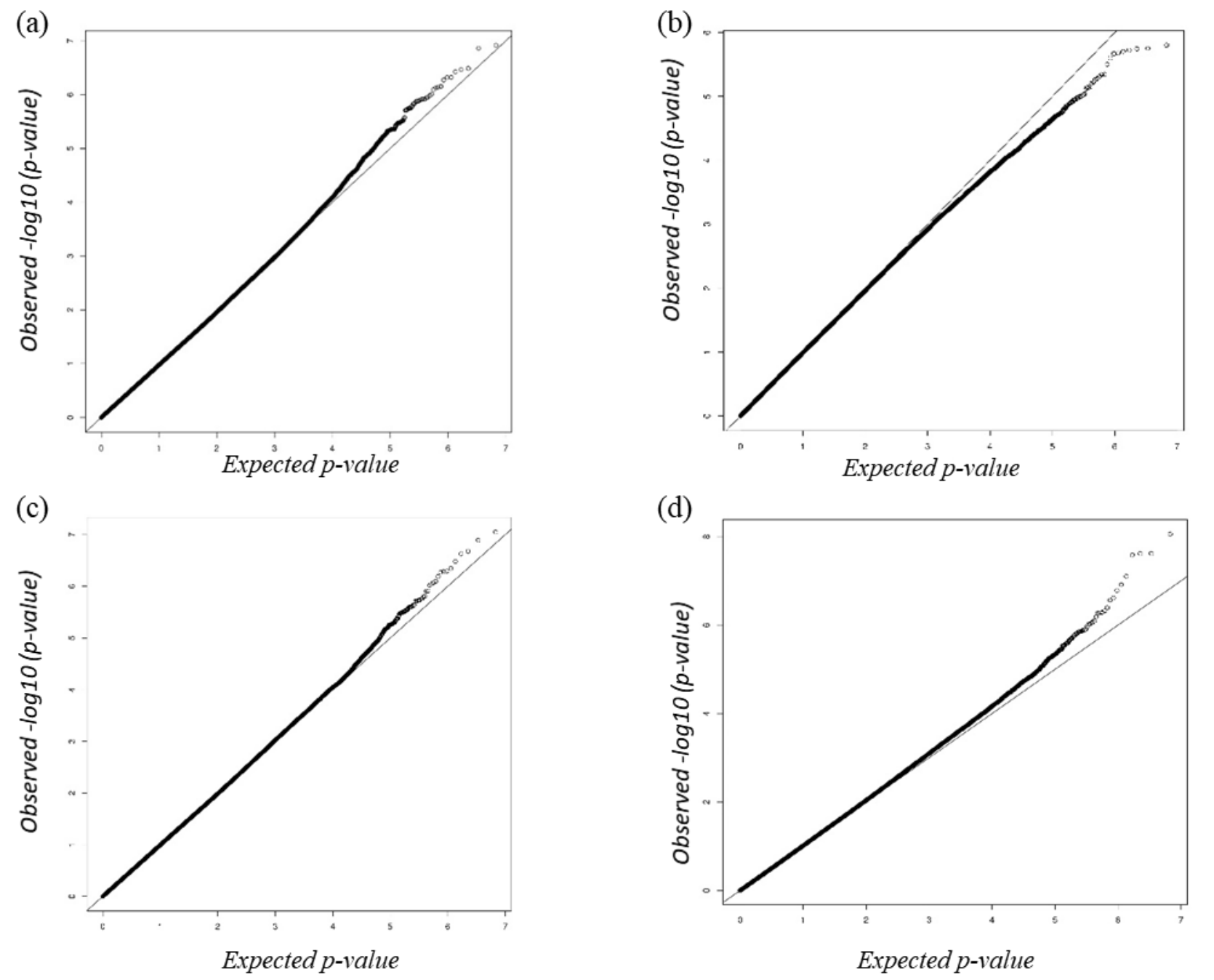

Supplementary Figure S 2.4 QQ-plot for single trait GWAS in P. trichocarpa for carbon isotope composition (a), leaf area (b), and stomatal density (c) and the corresponding multitrait GWAS with all 3 traits (d). For corresponding Manhattan Plots, see Figure 2.5a. 
(a)

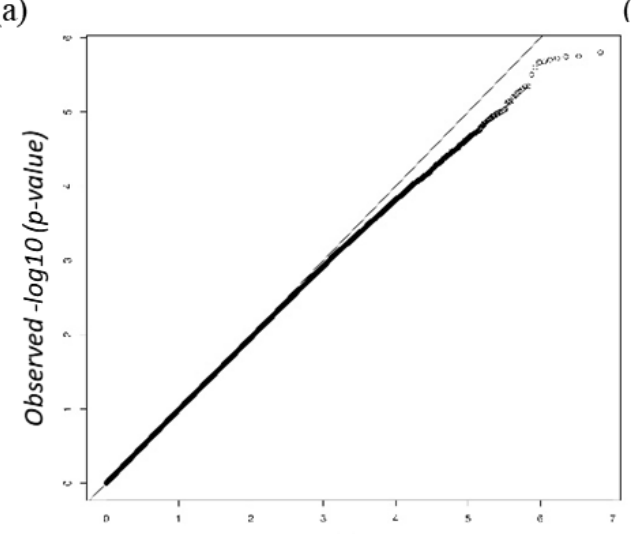

(c)

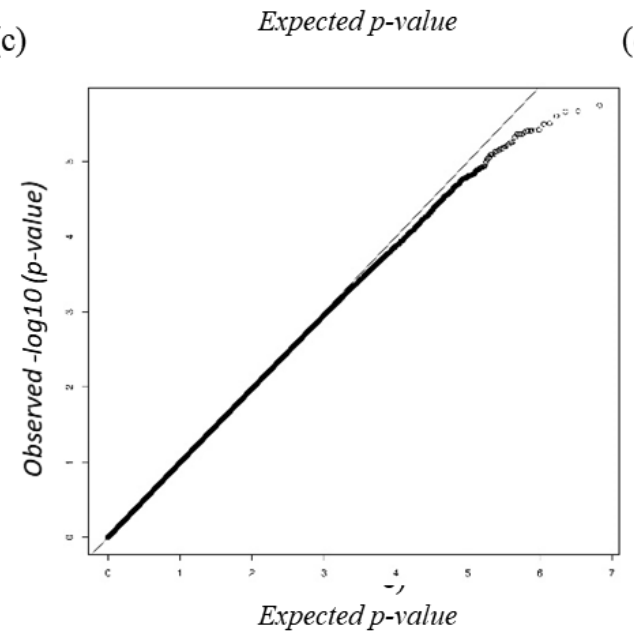

(e) (b)

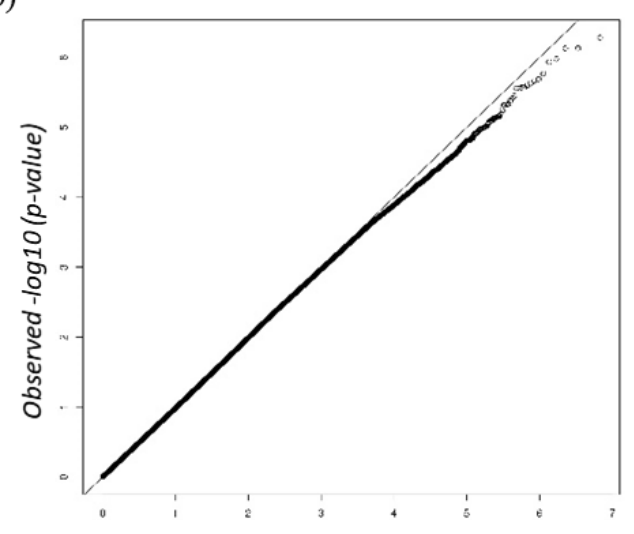

(d)

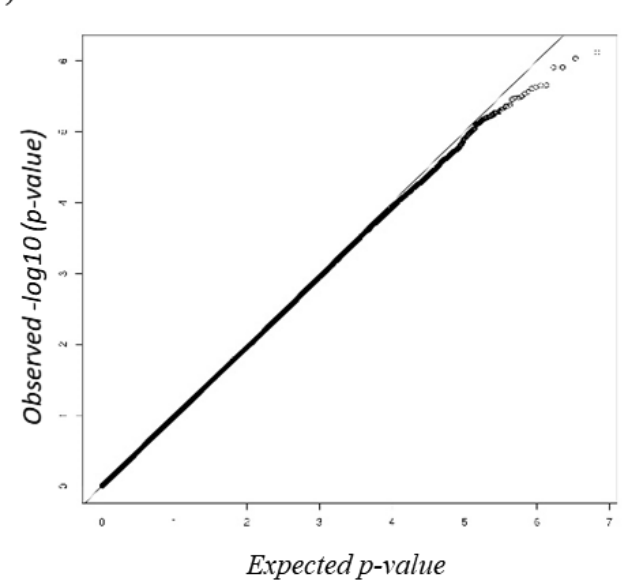

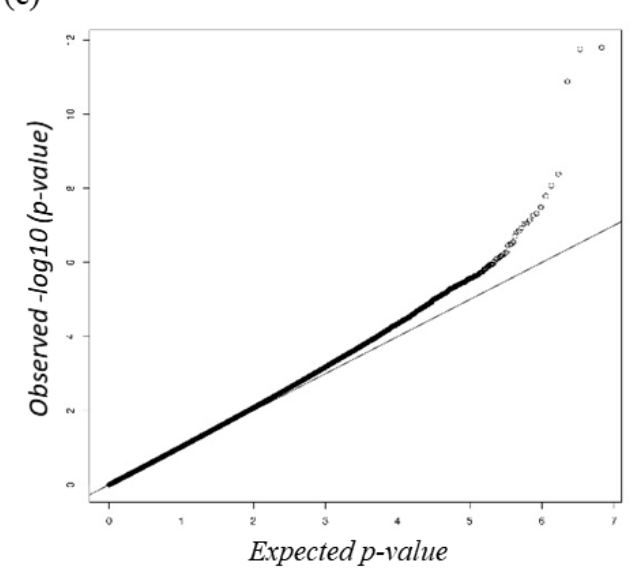

Supplementary Figure S 2.5 QQ-plot for single trait GWAS in P. trichocarpa for leaf area (a), leaf dry weight (b), leaf length (c) and leaf width (d) and the corresponding multitrait GWAS with all 4 leaf traits (e). See Figure 2.5c for the corresponding Manhattan plots. 

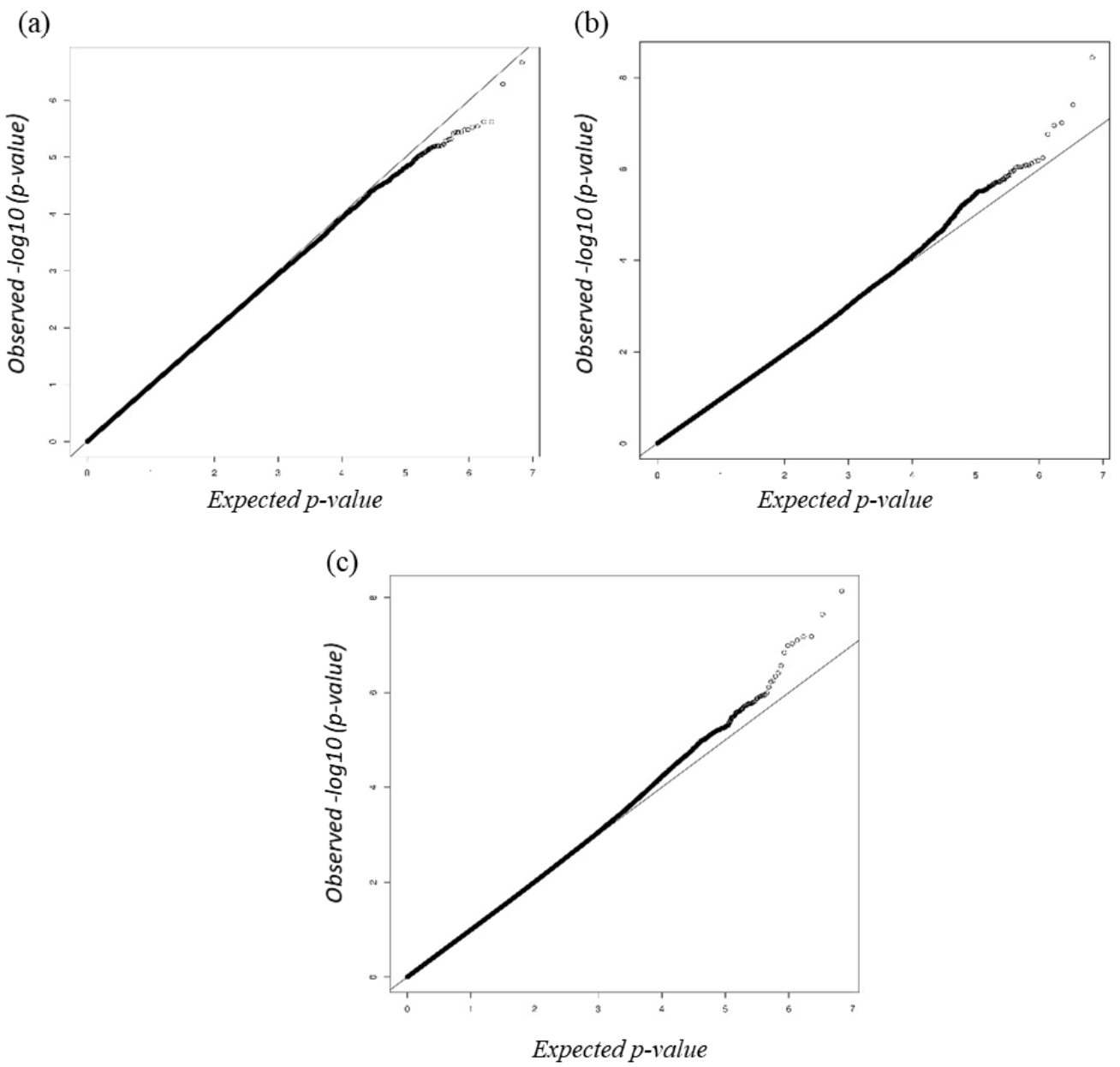

Supplementary Figure S 2.6 QQ-plot for single trait GWAS in P. trichocarpa for leaf aspect ratio (a) and specific leaf area (b), and the corresponding multitrait GWAS with both traits (c). See Figure 2.5e for the corresponding Manhattan plots. 
(a)

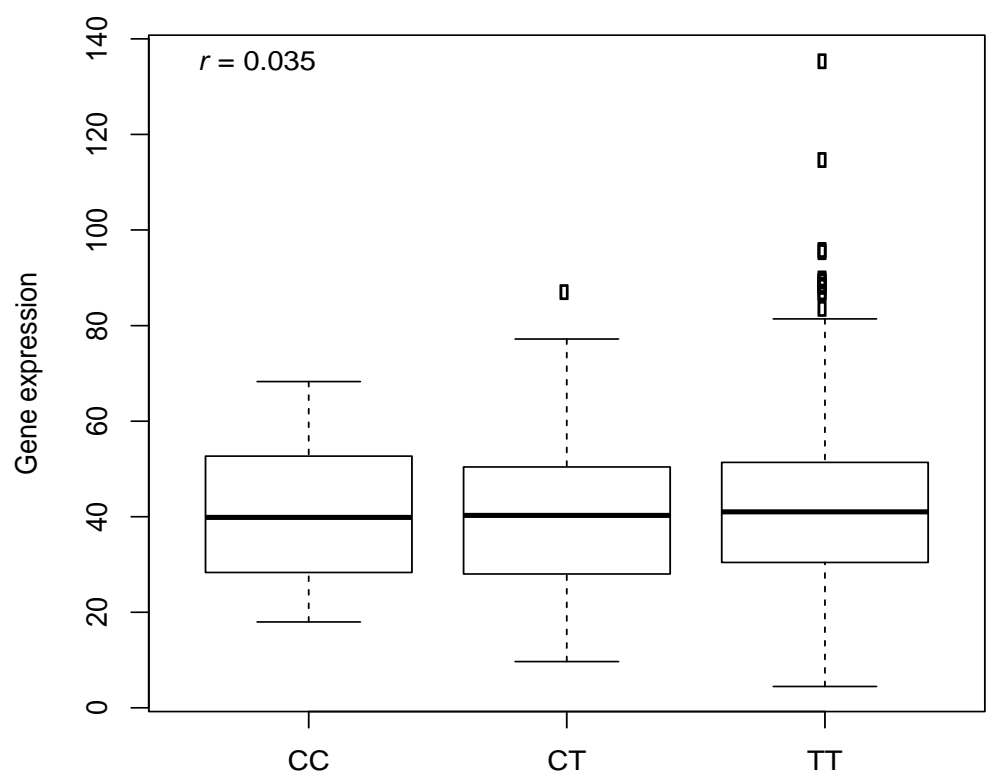

(b)

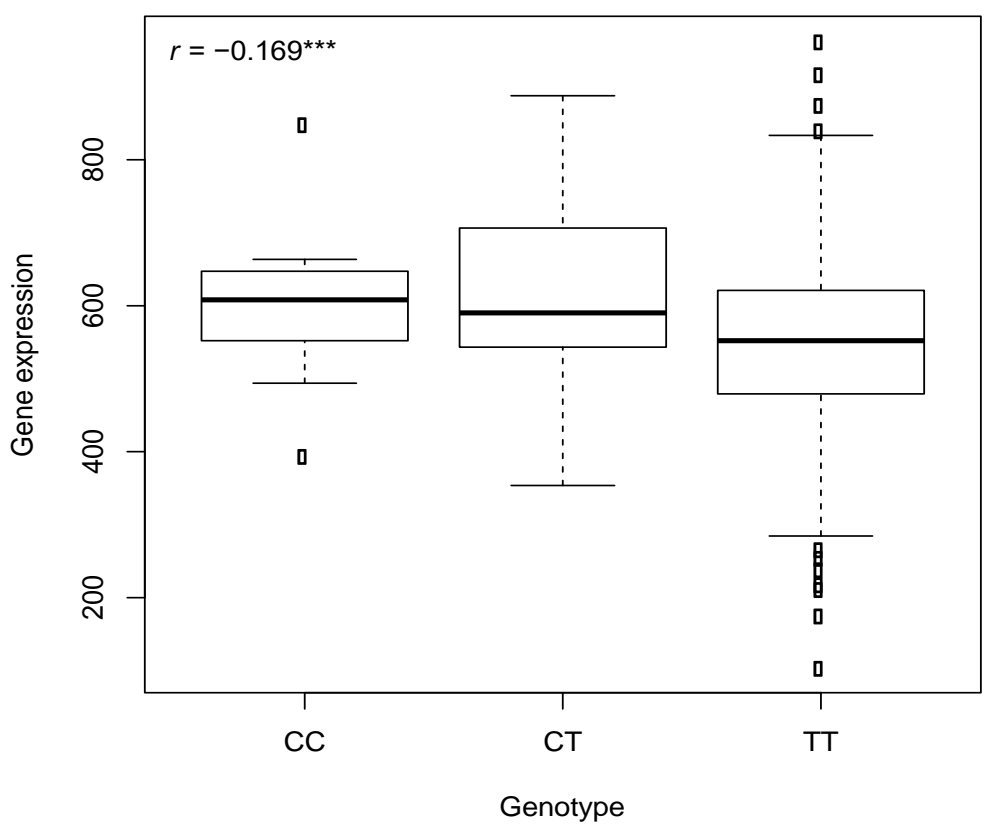

Supplementary Figure S 2.7 Pearson correlation of GAUT9 (Potri.004G111000) gene expression in (a) leaf and (b) developing xylem of $P$. trichocarpa with genotype at locus Chr04_9996091. Big boxes represent interquartile range with black horizontal bar within the box representing median expression 
value. The lower and upper whiskers represent 1.5 times the interquartile range, and the circles represent outliers.

(a)

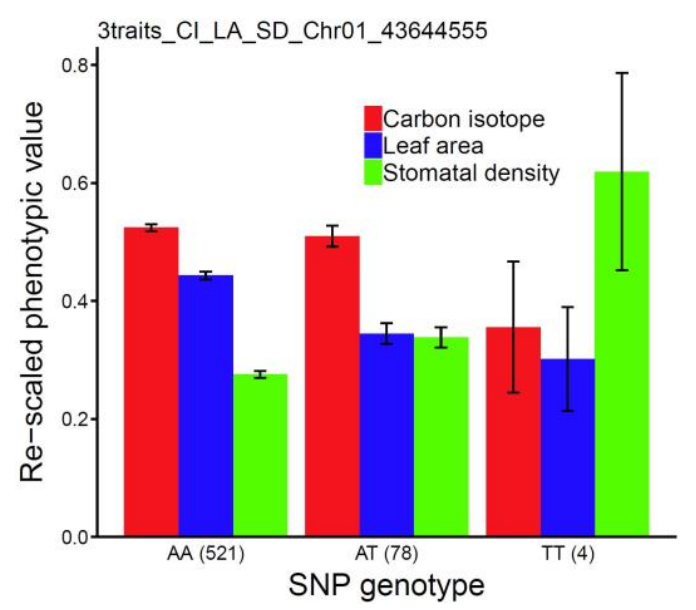

(c)

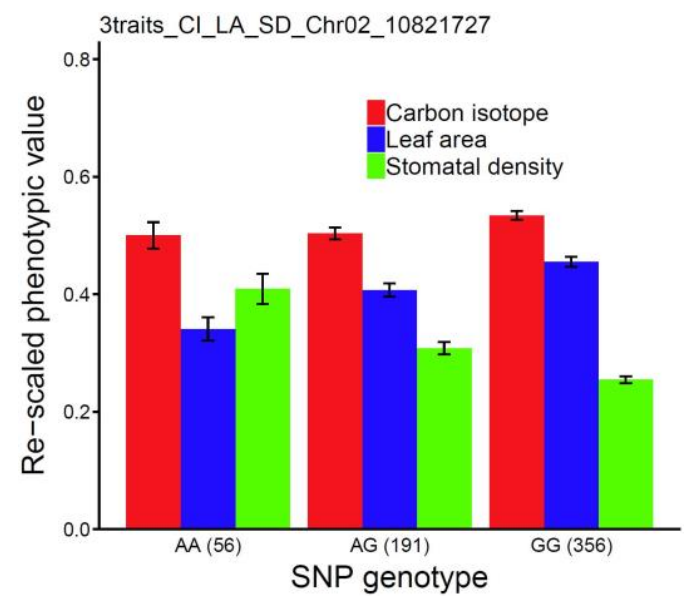

(b)

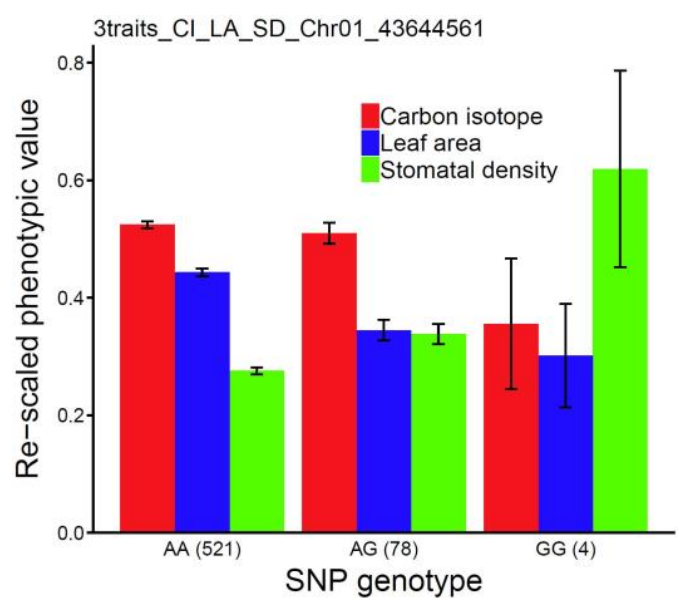

(d)

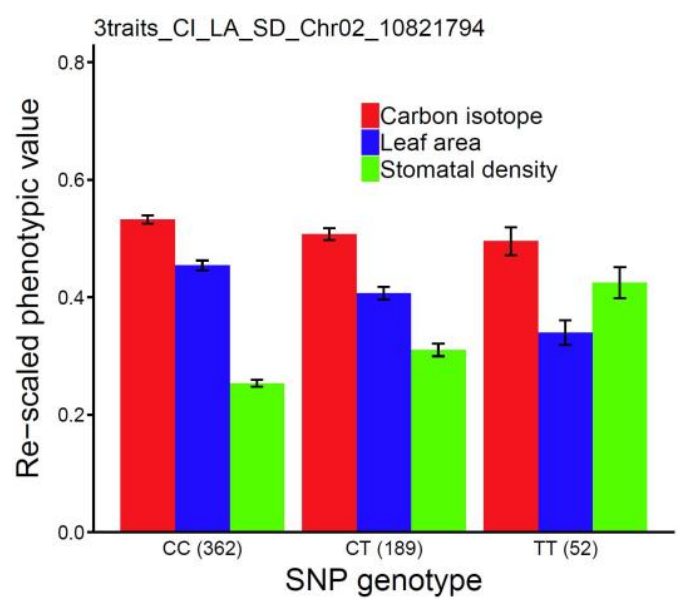

Supplementary Figure S 2.8 Allelic effects plots for single traits underlying the carbon isotope-leaf area-stomatal density multitrait association analysis in $P$. trichocarapa. Locus position is indicated above the plot. The error bars represent standard errors for re-scaled phenotypic values. (a) Locus Chr01_43644555; (b) Locus Chr01_43644561; (c) Locus Chr02_10821727; (d) Locus Chr02_10821794. 
(a)

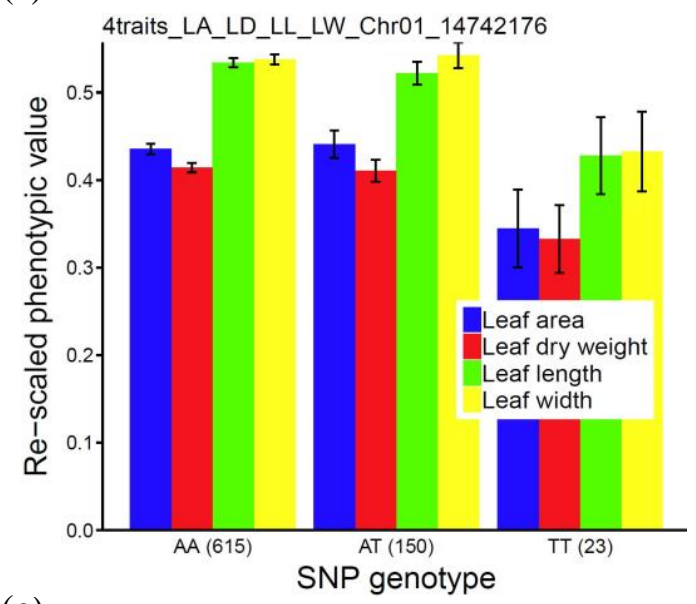

(c)

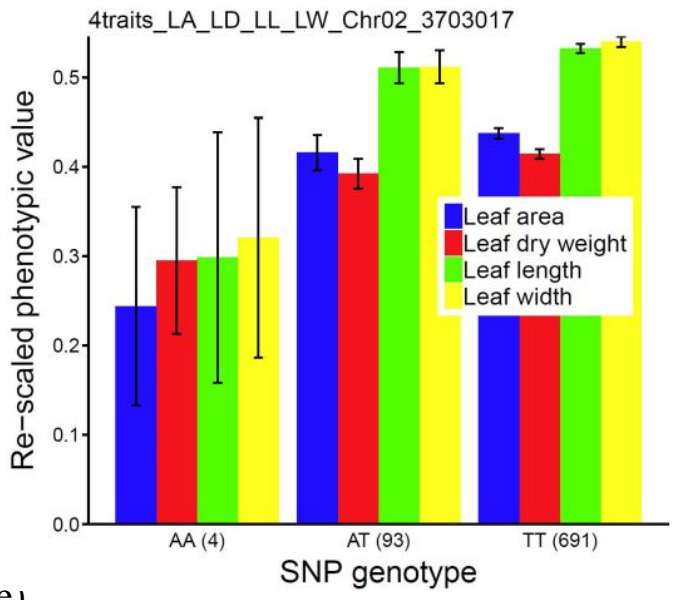

(e)

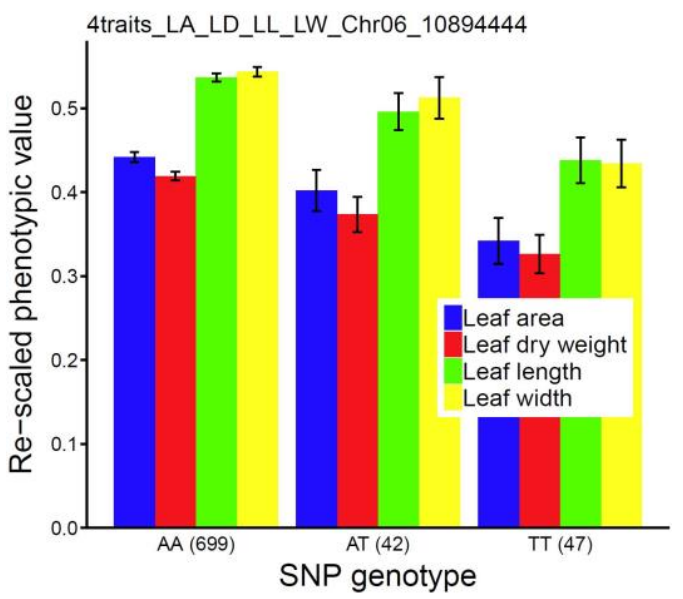

(b)

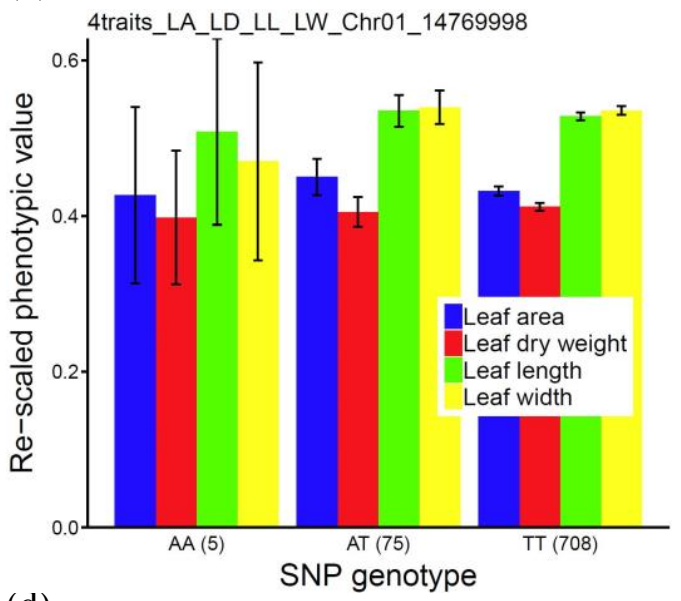

(d)

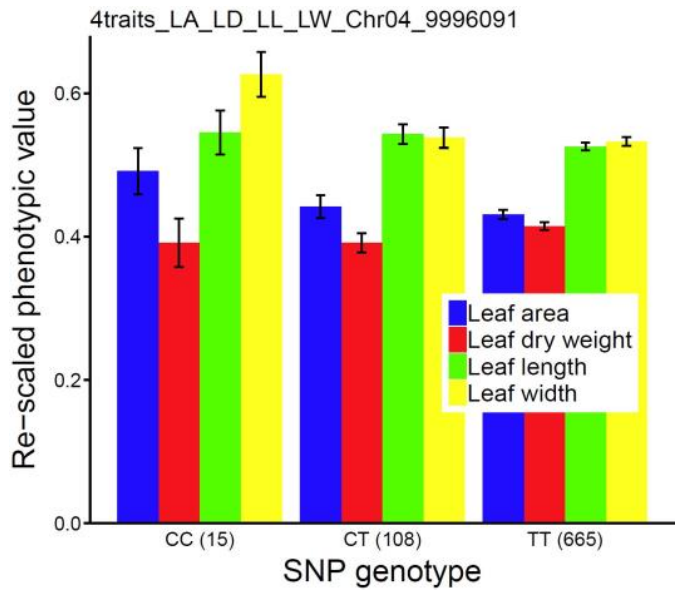

(f)

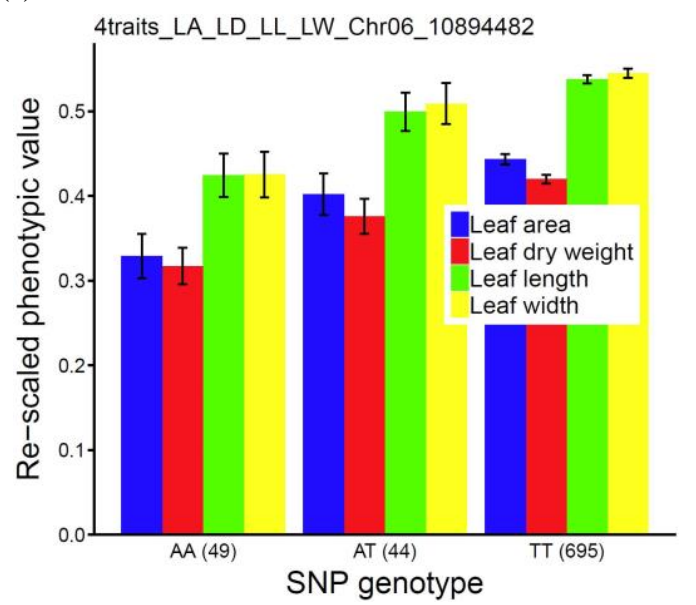


$(\mathrm{g})$

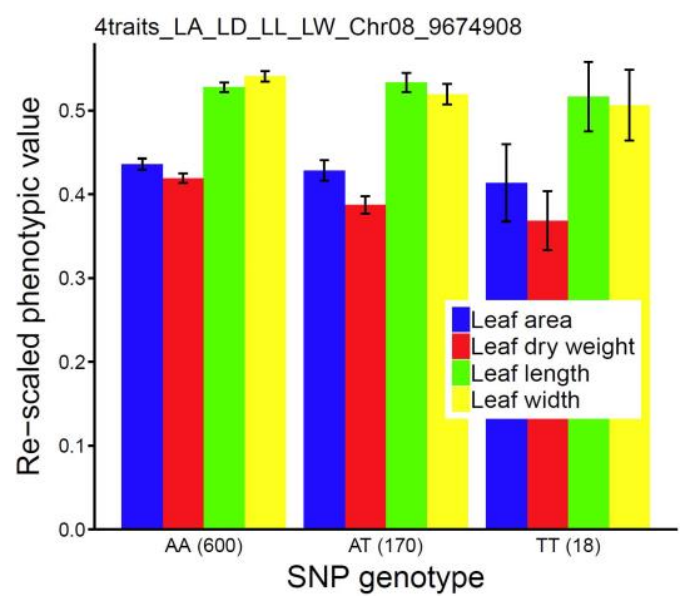

(i)

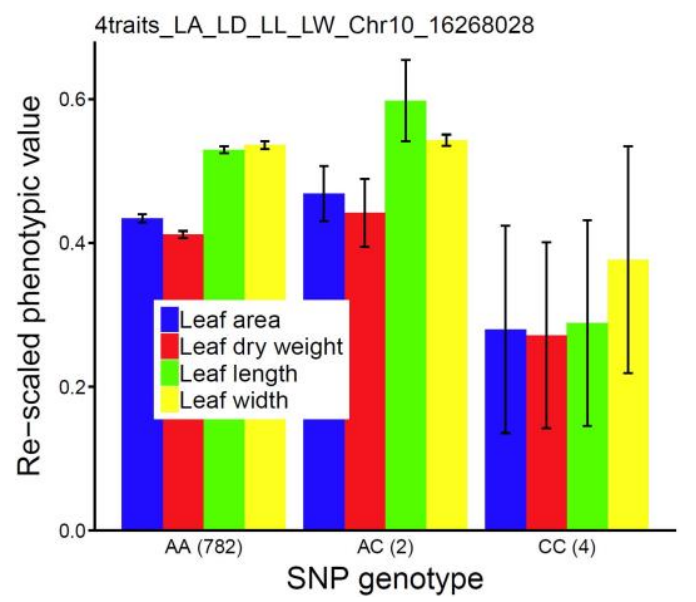

(h)

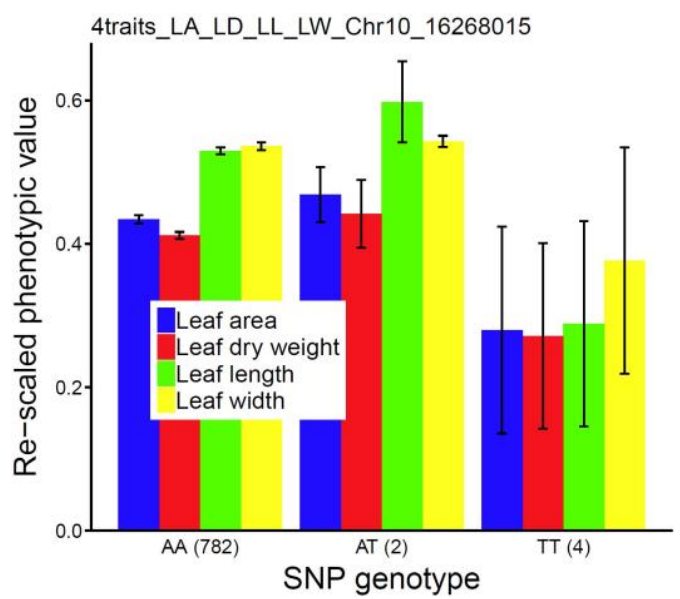

(j)

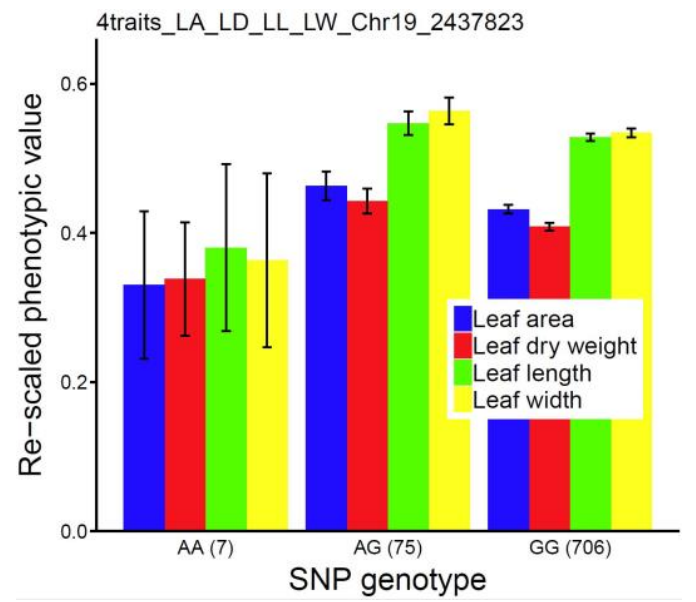

Supplementary Figure S 2.9 Allelic effects plots for single traits underlying the LA_LD_LL_LW association analysis in P. trichocarpa. Locus position is indicated above the plot. Numbers in parentheses below the plot are the number of observations of each genotype. Error bars represent standard errors for re-scaled phenotypic values. LA, leaf area; LD, leaf dry weight; LL, leaf length; LW, leaf width. (a) Locus Chr01_14742176; (b) Locus Chr01_14769998; (c) Locus Chr02_3703017; (d) Locus Chr04_9996091; (e) Locus Chr06_10894444; (f) Locus Chr06_10894482; (g) Locus Chr08_9674908; (h) Locus Chr10_16268015; (i) Locus Chr10_16268028; (j) Locus Chr19_2437823. 
(a)

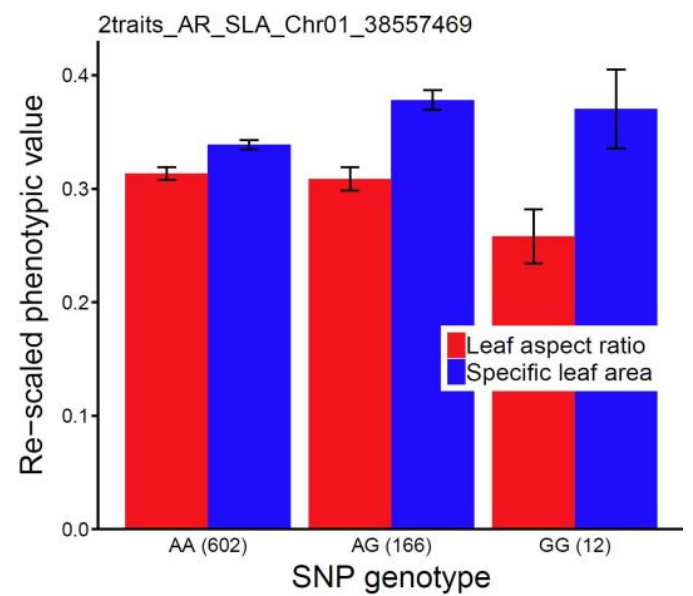

(c)

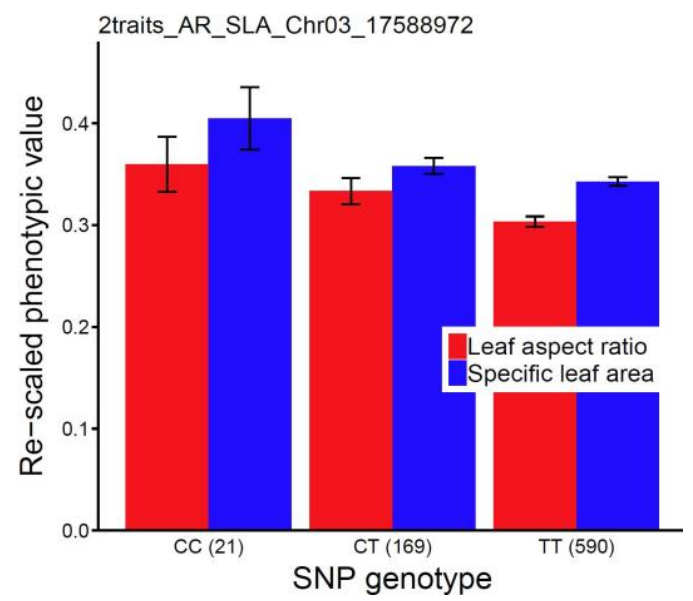

(b)

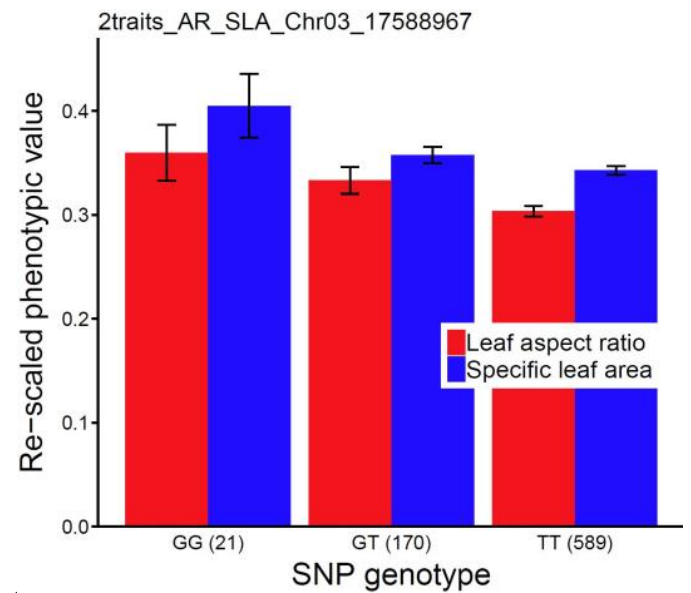

(d)

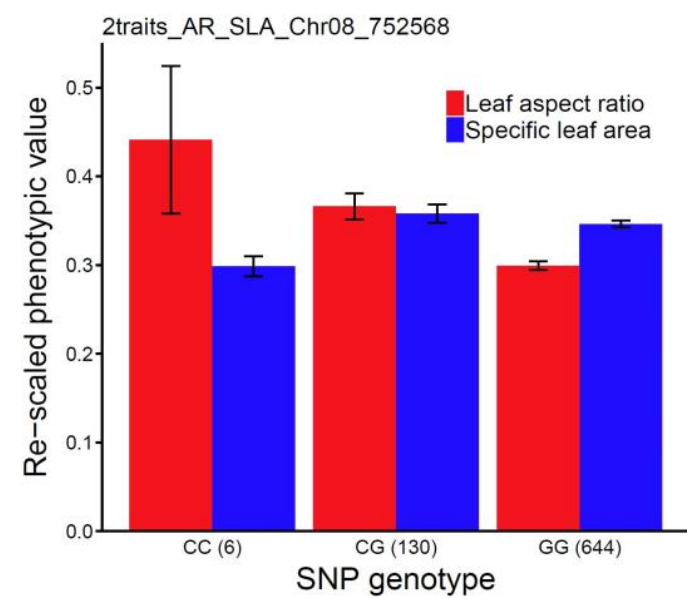

Supplementary Figure S 2.10 Allelic effects plots for single traits underlying the AR_SLA association analysis in P. trichocarpa. Locus position is indicated above the plot. Numbers in parentheses below the plot are the number of observations of each genotype. Error bars represent standard errors for re-scaled phenotypic values. AR, aspect ratio; SLA, specific leaf area. (a) Locus Chr01_38557469; (b) Locus Chr03_17588967; (c) Locus Chr03_17588972; (d) Locus Chr08_752568. 


\subsection{References}

Aitken SN, Yeaman S, Holliday JA, Wang T, Curtis-McLane S. 2008. Adaptation, migration or extirpation: climate change outcomes for tree populations. Evolutionary Applications 1: 95-111. Atmodjo MA, Hao Z, Mohnen D. 2013. Evolving Views of Pectin Biosynthesis. Annual Review of Plant Biology 64: 747-779.

Biswal AK, Atmodjo MA, Li M, Baxter HL, Yoo CG, Pu Y, Lee YC, Mazarei M, Black IM, Zhang JY, et al. 2018a. Sugar release and growth of biofuel crops are improved by downregulation of pectin biosynthesis. Nature Biotechnology 36: 249-257.

Biswal AK, Atmodjo MA, Pattathil S, Amos RA, Yang X, Winkeler K, Collins C, Mohanty SS, Ryno D, Tan L, et al. 2018b. Working towards recalcitrance mechanisms: Increased xylan and homogalacturonan production by overexpression of GAlactUronosylTransferase12 (GAUT12) causes increased recalcitrance and decreased growth in Populus. Biotechnology for Biofuels 11: 1-26.

Biswal AK, Hao Z, Pattathil S, Yang X, Winkeler K, Collins C, Mohanty SS, Richardson EA, Gelineo-Albersheim I, Hunt K, et al. 2015. Downregulation of GAUT12 in Populus deltoides by RNA silencing results in reduced recalcitrance, increased growth and reduced xylan and pectin in a woody biofuel feedstock. Biotechnology for Biofuels 8: 41.

Boyle EA, Li YI, Pritchard JK. 2017. An Expanded View of Complex Traits: From Polygenic to Omnigenic. Cell 169: 1177-1186.

Broadaway KA, Cutler DJ, Duncan R, Moore JL, Ware EB, Jhun MA, Bielak LF, Zhao W, Smith JA, Peyser PA, et al. 2016. A Statistical Approach for Testing Cross-Phenotype Effects of Rare Variants. American Journal of Human Genetics 98: 525-540.

Casale FP, Rakitsch B, Lippert C, Stegle O. 2015. Efficient set tests for the genetic analysis of correlated traits. Nature Methods 12: 755-758.

Chen C, Sun X, Duanmu H, Zhu D, Yu Y, Cao L, Liu A, Jia B, Xiao J, Zhu Y. 2015. GsCML27, a gene encoding a calcium-binding Ef-hand protein from Glycine soja, plays differential roles in plant responses to bicarbonate, salt and osmotic stresses. PLOS ONE 10: 1-19.

Drost DR, Puranik S, Novaes E, Novaes CRDB, Dervinis C, Gailing O, Kirst M. 2015. Genetical genomics of Populus leaf shape variation. BMC Plant Biology 15: 166.

Eckenwalder JE. 1996. Systematics and evolution of Populus. In: Stettler RF, Bradshaw HD, Heilman PE, Hinckley TM, eds. Biology of Populus and its Implications for Management and Conservation. Ottawa, Canada: NRC Research Press, 7-32.

Edel KH, Marchadier E, Brownlee C, Kudla J, Hetherington AM. 2017. The Evolution of Calcium- 
Based Signalling in Plants. Current Biology 27: R667-R679.

Elmore AJ, Craine JM, Nelson DM, Guinn SM. 2017. Continental scale variability of foliar nitrogen and carbon isotopes in Populus balsamifera and their relationships with climate. Scientific Reports 7: 7759.

Evans LM, Slavov GT, Rodgers-Melnick E, Martin J, Ranjan P, Muchero W, Brunner AM, Schackwitz W, Gunter L, Chen J-G, et al. 2014. Population genomics of Populus trichocarpa identifies signatures of selection and adaptive trait associations. Nature Genetics 46: 1089-1096.

Gao J, Lan T. 2016. Functional characterization of the late embryogenesis abundant (LEA) protein gene family from Pinus tabuliformis (Pinaceae) in Escherichia coli. Scientific Reports 6: 1-10.

Geraldes A, Farzaneh N, Grassa CJ, Mckown AD, Guy RD, Mansfield SD, Douglas CJ, Cronk

QCB. 2014. Landscape genomics of Populus trichocarpa: The role of hybridization, limited gene flow, and natural selection in shaping patterns of population structure. Evolution 68: 3260-3280.

Geraldes A, Pang J, Thiessen N, Cezard T, Moore R, Zhao Y, Tam A, Wang S, Friedmann M, Birol I, et al. 2011. SNP discovery in black cottonwood (Populus trichocarpa) by population transcriptome resequencing. Molecular Ecology Resources 11: 81-92.

González-martínez SC, Krutovsky K V, Neale DB. 2006. Forest-tree population genomics and adaptive evolution. New Phytologist 170: 227-238.

Goodstein DM, Shu S, Howson R, Neupane R, Hayes RD, Fazo J, Mitros T, Dirks W, Hellsten U, Putnam N, et al. 2012. Phytozome: A comparative platform for green plant genomics. Nucleic Acids Research 40: 1178-1186.

Gornall JL, Guy RD. 2007. Geographic variation in ecophysiological traits of black cottonwood (Populus trichocarpa). Canadian Journal of Botany 85: 1202-1213.

Hackinger S, Zeggini E. 2017. Statistical methods to detect pleiotropy in human complex traits. Open Biology 7: 170125.

Hernández I, Alegre L, Van Breusegem F, Munné-Bosch S. 2009. How relevant are flavonoids as antioxidants in plants? Trends in Plant Science 14: 125-132.

Holliday JA, Zhou L, Bawa R, Zhang M, Oubida RW. 2016. Evidence for extensive parallelism but divergent genomic architecture of adaptation along altitudinal and latitudinal gradients in Populus trichocarpa. New Phytologist 209: 1240-1251.

Ingvarsson PK, Hvidsten TR, Street NR. 2016. Towards integration of population and comparative genomics in forest trees. New Phytologist 212: 338-344.

Ingvarsson PK, Street NR. 2011. Association genetics of complex traits in plants. New Phytologist 189: 
909-922.

Jansson S, Douglas CJ. 2007. Populus: A Model System for Plant Biology. Annual Review of Plant Biology 58: 435-458.

Kim J, Zhang Y, Pan W. 2016. Powerful and adaptive testing for multi-trait and Multi-SNP associations with GWAS and sequencing data. Genetics 203: 715-731.

Lewit-Bentley A, Réty S. 2000. EF-hand calcium-binding proteins. Current Opinion in Structural Biology 10: 637-643.

MacDicken K, Jobsson O, Pina L, Marklund S, Maulo VC, Adhikari Y, Garzuglia M, Lindquist E, Reams G, Mundhenk P, et al. 2016. Global Forest Resources Assessment 2015. Rome: Food and Agriculture Organizations of the United Nations.

Magwanga RO, Lu P, Kirungu JN, Lu H, Wang X, Cai X, Zhou Z, Zhang Z, Salih H, Wang K, et al. 2018. Characterization of the late embryogenesis abundant (LEA) proteins family and their role in drought stress tolerance in upland cotton. BMC Genetics 19: 1-31.

McKown AD, Guy RD, Klápště J, Geraldes A, Friedmann M, Cronk QCB, El-Kassaby YA, Mansfield SD, Douglas CJ. 2014a. Geographical and environmental gradients shape phenotypic trait variation and genetic structure in Populus trichocarpa. New Phytologist 201: 1263-1276.

McKown AD, Guy RD, Quamme L, Klápště J, La Mantia J, Constabel CP, El-Kassaby YA, Hamelin RC, Zifkin M, Azam MS. 2014b. Association genetics, geography and ecophysiology link stomatal patterning in Populus trichocarpa with carbon gain and disease resistance trade-offs. Molecular Ecology 23: 5771-5790.

McKown AD, Klápště J, Guy RD, El-Kassaby YA, Mansfield SD. 2018. Ecological genomics of variation in bud-break phenology and mechanisms of response to climate warming in Populus trichocarpa. New Phytologist 220: 300-316.

McKown AD, Klapste J, Guy RD, Geraldes A, Porth I, Hannemann J, Friedmann M, Muchero W, Tuskan GA, Ehlting J, et al. 2014c. Genome-wide association implicates numerous genes underlying ecological trait variation in natural populations of Populus trichocarpa. New Phytologist 203: 535-553. Momayyezi M, Guy RD. 2017. Substantial role for carbonic anhydrase in latitudinal variation in mesophyll conductance of Populus trichocarpa Torr. \& Gray. Plant Cell and Environment 40: 138-149. Monclus R, Dreyer E, Delmotte FM, Villar M, Delay D, Boudouresque E, Petit JM, Marron N, Brechet C, Brignolas F. 2005. Productivity, leaf traits and carbon isotope discrimination in 29 Populus deltoides $\times$ P. nigra clones. New Phytologist 167: 53-62.

Monclus R, Villar M, Barbaroux C, Bastien C, Fichot R, Delmotte FM, Delay D, Petit JM, Brchet 
C, Dreyer E, et al. 2009. Productivity, water-use efficiency and tolerance to moderate water deficit correlate in 33 poplar genotypes from a Populus deltoides $\times$ Populus trichocarpa F1progeny. Tree Physiology 29: 1329-1339.

Neale DB, Kremer A. 2011. Forest tree genomics: Growing resources and applications. Nature Reviews Genetics 12: 111-122.

Neale DB, Savolainen O. 2004. Association genetics of complex traits in conifers. New Phytologist 9 : 325-330.

Nishikubo N, Takahashi J, Roos AA, Derba-Maceluch M, Piens K, Brumer H, Teeri TT, Stalbrand H, Mellerowicz EJ. 2011. Xyloglucan endo-Transglycosylase-Mediated Xyloglucan Rearrangements in Developing Wood of Hybrid Aspen. Plant Physiology 155: 399-413.

Parvez MM, Tomita-Yokotani K, Fujii Y, Konishi T, Iwashina T. 2004. Effects of quercetin and its seven derivatives on the growth of Arabidopsis thaliana and Neurospora crassa. Biochemical Systematics and Ecology 32: 631-635.

Porter HF, O'Reilly PF. 2017. Multivariate simulation framework reveals performance of multi-trait GWAS methods. Scientific Reports 7: 1-12.

Ranty B, Aldon D, Cotelle V, Galaud J-P, Thuleau P, Mazars C. 2016. Calcium Sensors as Key Hubs in Plant Responses to Biotic and Abiotic Stresses. Frontiers in Plant Science 7: 1-7.

Ritchie MD, Holzinger ER, Li R, Pendergrass SA, Kim D. 2015. Methods of integrating data to uncover genotype-phenotype interactions. Nature Reviews Genetics 16: 85-97.

Rubin EM. 2008. Genomics of cellulosic biofuels. Nature 454: 841-845.

Rui Y, Xiao C, Yi H, Kandemir B, Wang JZ, Puri VM, Anderson CT. 2017.

POLYGALACTURONASE INVOLVED IN EXPANSION3 Functions in Seedling Development, Rosette Growth, and Stomatal Dynamics in Arabidopsis thaliana. The Plant Cell 29: 2413-2432.

Sanders D, Pelloux J, Brownlee C, Harper JF. 2002. Calcium at the Crossroads of Signaling. The Plant Cell 14: S401-S417.

Schindelin J, Rueden CT, Hiner MC, Eliceiri KW. 2015. The ImageJ ecosystem: An open platform for biomedical image analysis. Molecular Reproduction and Development 82: 518-529.

Scholander APF, Hammel HT, Bradstreet ED, Hemmingsen EA. 2016. Sap Pressure in Vascular Plants. Science 148: 339-346.

Sham PC, Purcell SM. 2014. Statistical power and significance testing in large-scale genetic studies. Nature Reviews Genetics 15: 335-346.

Shannon P, Markiel A, Owen Ozier 2, Baliga NS, Wang JT, Ramage D, Amin N, Schwikowski B, 
Ideker T. 2003. Cytoscape: a software environment for integrated models of biomolecular interaction networks. Genome Research 13: 2498-2504.

Shim H, Chasman DI, Smith JD, Mora S, Ridker PM, Nickerson DA, Krauss RM, Stephens M. 2015. A multivariate genome-wide association analysis of $10 \mathrm{LDL}$ subfractions, and their response to statin treatment, in 1868 Caucasians. PLoS ONE 10: 1-20.

Sjödin A, Street NR, Sandberg G, Gustafsson P, Jansson S. 2009. The Populus Genome Integrative Explorer (PopGenIE): A new resource for exploring the Populus genome. New Phytologist 182: 10131025.

Slavov GT, Difazio SP, Martin J, Schackwitz W, Muchero W, Rodgers-Melnick E, Lipphardt MF, Pennacchio CP, Hellsten U, Pennacchio LA, et al. 2012. Genome resequencing reveals multiscale geographic structure and extensive linkage disequilibrium in the forest tree Populus trichocarpa. New Phytologist 196: 713-725.

Solovieff N, Cotsapas C, Lee PH, Purcell SM, Smoller JW. 2013. Pleiotropy in complex traits: Challenges and strategies. Nature Reviews Genetics 14: 483-495.

Soolanayakanahally RY, Guy RD, Silim SN, Drewes EC, Schroeder WR. 2009. Enhanced assimilation rate and water use efficiency with latitude through increased photosynthetic capacity and internal conductance in balsam poplar (Populus balsamifera L.). Plant, Cell and Environment 32: 18211832.

Soolanayakanahally RY, Guy RD, Street NR, Robinson KM, Silim SN, Albrectsen BR, Jansson S. 2015. Comparative physiology of allopatric Populus species: geographic clines in photosynthesis, height growth, and carbon isotope discrimination in common gardens. Frontiers in Plant Science 6: 528.

Stephens M. 2013. A Unified Framework for Association Analysis with Multiple Related Phenotypes. PLoS ONE 8: e65245.

Sterling JD, Atmodjo MA, Inwood SE, Kumar Kolli VS, Quigley HF, Hahn MG, Mohnen D. 2006. Functional identification of an Arabidopsis pectin biosynthetic homogalacturonan galacturonosyltransferase. Proceedings of the National Academy of Sciences 103: 5236-5241. Tschaplinski TJ, Abraham PE, Jawdy SS, Gunter LE, Martin MZ, Engle NL, Yang X, Tuskan GA. 2019. The nature of the progression of drought stress drives differential metabolomic responses in Populus deltoides. Annals of Botany In press.

Tschaplinski TJ, Standaert RF, Engle NL, Martin MZ, Sangha AK, Parks JM, Smith JC, Samuel R, Jiang N, Pu Y, et al. 2012. Down-regulation of the caffeic acid O-methyltransferase gene in switchgrass reveals a novel monolignol analog. Biotechnology for Biofuels 5: 71. 
Tuskan GA, DiFazio S, Jansson S, Bohlmann J, Grigoriev I, Hellsten U, Putnam N, Ralph S, Rombauts S, Salamov A, et al. 2006. The genome of black cottonwood, Populus trichocarpa (Torr. \& Gray). Science 313: 1596.

Vining KJ, Pomraning KR, Wilhelm LJ, Priest HD, Pellegrini M, Mockler TC, Freitag M, Strauss SH. 2012. Dynamic DNA cytosine methylation in the Populus trichocarpa genome: Tissue-level variation and relationship to gene expression. BMC Genomics 13: 27.

Visscher PM, Wray NR, Zhang Q, Sklar P, McCarthy MI, Brown MA, Yang J. 2017. 10 Years of GWAS Discovery: Biology, Function, and Translation. American Journal of Human Genetics 101: 5-22. Voiniciuc C, Engle KA, Günl M, Dieluweit S, Schmidt MH-W, Yang J-Y, Moremen KW, Mohnen D, Usadel B. 2018. Identification of Key Enzymes for Pectin Synthesis in Seed Mucilage. Plant Physiology 178: 1045-1064.

Weigel D, Nordborg M. 2015. Population Genomics for Understanding Adaptation in Wild Plant Species. Annual review of genetics: 1-24.

Weighill D, Jones P, Shah M, Ranjan P, Muchero W, Schmutz J, Sreedasyam A, Macaya-Sanz D, Sykes R, Zhao N, et al. 2018. Pleiotropic and Epistatic Network-Based Discovery: Integrated Networks for Target Gene Discovery. Frontiers in Energy Research 6: 30.

Wheeler NC, Steiner KC, Schlarbaum SE, Neale DB. 2015. The Evolution of Forest Genetics and Tree Improvement Research in the United States. Journal of Forestry 113: 500-510.

Yang J, Lee SH, Goddard ME, Visscher PM. 2011. GCTA: A tool for genome-wide complex trait analysis. American Journal of Human Genetics 88: 76-82.

Yang X, Li X, Li B, Zhang D. 2014. Identification of Genes Differentially Expressed in Shoot Apical Meristems and in Mature Xylem of Populus tomentosa. Plant Molecular Biology Reporter 32: 452-464. Zeng H, Zhang Y, Zhang X, Pi E, Zhu Y. 2017. Analysis of EF-Hand Proteins in Soybean Genome Suggests Their Potential Roles in Environmental and Nutritional Stress Signaling. Frontiers in Plant Science 8: 1-15.

Zhang C, Du X, Tang K, Yang Z, Pan L, Zhu P, Luo J, Jiang Y, Zhang H, Wan H, et al. 2018 a. Arabidopsis AGDP1 links H3K9me2 to DNA methylation in heterochromatin. Nature Communications 9: 4547 .

Zhang J, Yang Y, Zheng K, Xie M, Feng K, Jawdy SS, Gunter LE, Ranjan P, Singan VR, Engle N, et al. 2018b. Genome-wide association studies and expression-based quantitative trait loci analyses reveal roles of HCT2 in caffeoylquinic acid biosynthesis and its regulation by defense-responsive transcription factors in Populus. New Phytologist 220: 502-516. 
Zhou X, Stephens M. 2012. Genome-wide efficient mixed model analysis for association studies. Nature Genetics 44: 821-824.

Zhou X, Stephens M. 2014. Efficient Algorithms for Multivariate Linear Mixed Models in Genome-wide Association Studies. Nature Methods 11: 407-409.

Zhu JK. 2016. Abiotic Stress Signaling and Responses in Plants. Cell 167: 313-324. 


\section{Chapter 3. Local adaptation in Populus trichocarpa}

\subsection{Abstract}

Unraveling the complex patterns of adaptation in forest ecosystems by exploiting the association between genetic and environmental heterogeneity across populations is of increasing interest. Clear understanding of local adaptation can help in effective optimization of productivity and stress tolerance traits, sustainable use and management of forest resources, conservation strategies and domestication of commercial species. Here we integrated genomic, geoclimatic and phenotypic data and used three conceptually different methods for testing the evidence for local adaptation in Populus trichocarpa: (1) correlation of phenotypic traits with geoclimatic variables; (2) genotype-environment association (GEA) analysis; and (3) a multivariate ordination method called redundancy analysis (RDA). We identified genomic outlier loci and underscored the relative contribution of climate and geography on neutral population structure and the relative contribution of climate, geography and genomic data (SNPs) on adaptive traits. Out of 67 genes identified based on outlier loci in GEA, 32 of them were shared with the genes identified from climate and phenotypic RDA models. Climate and geography matrices individually explained $2.57 \%$ and $5.66 \%$ of the variation, respectively in the SNP eigenvector matrix that defines neutral population structure in the climate RDA model. Similarly, individual SNP eigenvector, climate and geography predictor variable matrices explained $21.66 \%, 12.31 \%$ and $16.63 \%$ of the variance in the adaptive trait matrix in the phenotypic RDA model. Based on these approaches we identified multiple loci conferring local adaption including anthocyanin and fatty acid pathways related genes that are involved in biotic and abiotic stress tolerance, defense and growth and development mechanisms in plants. This study provides invaluable insights on the power of using multivariate methods in integrating genomic, geoclimatic and phenotypic data to unravel the genetic mechanisms underlying local adaption in $P$. trichocarpa. The findings from this study can be useful for developing conservation strategies and management of forest resources and complex trait optimization for commercial use.

\subsection{Introduction}

Understanding the complex patterns of adaptation in forest ecosystems is of increasing interest (Bragg, Supple, Andrew, \& Borevitz, 2015; Rellstab, Gugerli, Eckert, Hancock, \& Holderegger, 2015; Sork et al., 2013). Natural selection has a great role in shaping the patterns of genetic diversity and adaptation. Genetic differentiation leading to local adaptation depends on the strength of selection, biotic and abiotic factors, the relative roles of selection and gene flow, demographic history, and spatial and environmental variation. Knowledge of spatial distribution of adaptive genetic variation and its influence on morphological, phenological and physiological traits is crucial in the face of climate change (Neale \& Kremer, 2011). Clear understanding of local adaptation can help in effective optimization of productivity 
and stress tolerance traits, sustainable use and management of forest resources, conservation strategies and domestication of commercial species. Forest ecosystems are at risk and long-lived immobile organisms like trees respond much slower to the changing climate regime and may suffer adaptational lag (Aitken, Yeaman, Holliday, Wang, \& Curtis-McLane, 2008). However, the mechanisms of local adaptation are not very well understood.

Forest trees are excellent model systems for studying local adaptation. Forest trees have long generation times, harbor high genetic and phenotypic variation, and often cover a large range of environments and show geographic clines for adaptive traits (Neale \& Kremer, 2011; Savolainen, Lascoux, \& Merilä, 2013). Spatial patterns of genetic variation are affected by the interaction among habitat heterogeneity (geographic, edaphic and environmental), migration and selection (Richardson, Urban, Bolnick, \& Skelly, 2014). Fitness related traits are affected by the shift in allele frequencies due to local selective forces, which can disrupt local adaptation (Vangestel, Eckert, Wegrzyn, St. Clair, \& Neale, 2018). Despite the belief that the gene flow homogenizes the populations by swamping the adaptive alleles, there is evidence that forest trees exhibit local adaptation at the fine spatial scales (Eckert et al., 2015; Slavov et al., 2009; Slavov \& Zhelev, 2010). Local adaption with gene flow is particularly effective when selection is strong, and the environment is heterogeneous (Lind et al., 2017).

Local adaptation is the tendency of plant populations to show higher fitness in their native environment compared to a foreign habitat. Various methods are in use for understanding the mechanisms of local adaptation: 1) common garden experiments, especially reciprocal transplant experiments that test for the performance of all accessions in all of the sampled home environments; 2) Phenotype-environment correlations; 3) Genetic differentiation (outlier detection) methods that solely require genomic data at the population level; 4) GEA methods that consider associations between genomic and environmental data; and 5) Multivariate ordination methods that simultaneously test for the association of loci with predictor variables. Reciprocal transplant experiments are the classical way for testing whether the genotypes do better in their native environments compared to foreign environments. Furthermore, common gardens in general are useful for unraveling the genetic basis of quantitative traits, for testing the effects of the environment on genotypes and comparing phenotypic differentiation with neutral population genetic variation. However, recent work on local adaptation is more focused on genetic differentiation and GEA methods for identifying the loci conferring local adaptation (Lind, Menon, Bolte, Faske, \& Eckert, 2018).

Outlier detection methods mainly differ in their computational efficiency and the type of data they can use (reviewed in Capblancq, Luu, Blum, \& Bazin, 2018; Forester, Lasky, Wagner, \& Urban, 2018; Lind et al., 2018). The accuracy of these methods depends on the sample size, the nature of the data and the model used to correct for spatial variation (Lotterhos \& Whitlock, 2015). The main difficulty is to distinguish true selection signatures from false positives due to population structure and demographic 
history such as population expansion, population bottleneck and isolation by distance (Jensen, Kim, DuMont, Aquadro, \& Bustamante, 2005; Meirmans, 2012). Moreover, it should be noted that there is no universal method that works for all sample sizes and data types. Differentiation outlier methods (genome scans) allow for detecting loci with strong allele frequency differences among populations without needing environmental or phenotypic data (Evans et al., 2014), although there is a discordance among results of different gene scan methods. In contrast, GEA methods identify loci based on the associations between genetic and environmental data that are potential drivers of selection. This allows for individual level sampling in contrast to the population level sampling in the differentiation methods, thereby providing an explicit link between the genomic loci and predictor variable (for e.g. environmental variable). However, these methods can suffer from high false-positive rates if not correctly controlled for multiple testing (De Mita et al., 2013; Meirmans, 2012). Univariate mixed-effect models that test for the association of one locus at a time with the predictor variables are the most popular methods in this class (Frichot, Schoville, Bouchard, \& François, 2013; Joost et al., 2007; Stucki et al., 2017).

Recently, GEA and related methods have gained popularity in the field of landscape genomics. The main goal of landscape genomics is to use genomic data to determine the relative effects of demographic history, environmental heterogeneity, selection and migration on adaptive evolutionary processes (Sork et al., 2013; Storfer, Patton, \& Fraik, 2018). However, most of the current landscape genomic studies have limited power, largely due to limited sample size. Because of this, there is not much congruence across studies in terms of the adaptive loci identified in forest trees (Ćalić, Bussotti, MartínezGarcía, \& Neale, 2016). Such discrepancies across studies could reflect parallel evolution (Geraldes et al., 2014), but robust conclusions are limited due to the low power for detecting adaptive loci in the studies conducted thus far (Ćalić et al., 2016). In addition to large sample sizes, robust landscape genomics inferences require homogeneous sampling from across the range of the species distribution and refined computationally efficient methods. Furthermore, combining common garden studies with landscape genomics is a better approach for validation of candidate genes and increased power of detection (Bragg et al., 2015; De Villemereuil, Gaggiotti, Mouterde, \& Till-Bottraud, 2016; Flanagan, Forester, Latch, Aitken, \& Hoban, 2018; Prunier, Verta, \& Mackay, 2016). Power and inference can also be improved by integrating genotype, phenotype and environment data. Furthermore, multivariate approaches that analyze many loci simultaneously may provide better estimation of the correlations with predictor variables. These methods are effective in identifying multilocus selection outliers as they test for groups of markers covarying in response to environmental predictors. Furthermore, whenever possible, integrated approaches that take into account of genomic, environmental and phenotypic data from the same population sample are most desirable (Eckert et al., 2015; Talbot et al., 2017; Vangestel et al., 2018). 
Recently, the ordination method known as Redundancy Analysis (RDA) has gained popularity for analyzing multivariate genomic, geoclimatic and phenotypic data (Brauer, Hammer, \& Beheregaray, 2016; Capblancq et al., 2018; Forester, Jones, Joost, Landguth, \& Lasky, 2016; Forester et al., 2018; Gugger, Liang, Sork, Hodgskiss, \& Wright, 2018; Hofmeister, Werner, \& Lovette, 2019; Lasky et al., 2012; Meirmans, 2015; Meirmans, Godbout, Lamothe, Thompson, \& Isabel, 2017; Rellstab et al., 2015; Sork et al., 2016; Talbot et al., 2017). RDA is widely used in community ecological studies, but its application in the field of landscape genomics is relatively recent, though it is probably the best method for integrating all of this information (Eckert et al., 2015; Vangestel et al., 2018). RDA is a family of ordination methods that allow for the representation of complex biological information in a reduced number of dimensions. Indirect ordination methods like PCA are used for reducing the dimensionality of a set of data (Cavalli-Sforza, 1996), but they do not explicitly take predictors into account. Direct ordination methods like RDA account for predictor variables and are effective at detecting multilocus selection by testing how a group of SNPs covary in response to environmental predictors (Rellstab et al., 2015). RDA uses constrained ordination to model a set of predictor variables such that it restricts the decomposed orthogonal response matrix to the combinations of predictors (Jombart, Pontier, \& Dufour, 2009; Legendre \& Legendre, 2012) and uses unconstrained ordination axes to model the response data (e.g. genetic variation). RDA allows for the detection of weak multilocus selection signals while controlling for false positives (Le Corre \& Kremer, 2012; Savolainen et al., 2013; Tiffin \& Ross-Ibarra, 2014). It not only allows detection of loci under selection but also allows for partitioning the variance in the response matrix into predictor variables (redundant variance). RDA provides greater statistical power than PCA based genome scan methods (Capblancq et al., 2018), outlier differentiation methods, GEA methods and other multivariate methods such as random forests, and works well for a range of sample sizes, selection levels, demographic histories and sampling designs (Forester et al., 2018). An advantage of outlier analysis in RDA is it allows identification of loci correlated with the multivariate environmental gradients experienced by plants that may be important to local adaptation, as opposed to testing climate variables individually (Hancock et al., 2011).

With ever growing genomic data and the improvement in genome scan and other differentiation and GEA methods, it is increasingly feasible to determine the loci conferring local adaptation. Several genes controlling cold-hardiness in conifers have been identified using association genetics, differentiation and GEA methods (Eckert et al., 2010; Evans et al., 2014; Vangestel et al., 2018). Some studies have also taken advantage of common garden studies where outlier loci identified from genome scan methods have been shown to control physiological and phenological traits (Capblancq et al., 2018; Evans et al., 2014; Gonzales-Vigil, Hefer, von Loessl, La Mantia, \& Mansfield, 2017). Hundreds of genomic regions showing evidence of recent positive and/or divergent selection and enrichment for 
associations with adaptive traits that displayed patterns consistent with natural selection have been identified in Populus trichocarpa (Evans et al., 2014; Geraldes et al., 2014; Holliday, Zhou, Bawa, Zhang, \& Oubida, 2016; Zhou, Bawa, \& Holliday, 2014). The generally low LD in forest trees warrants the use of high density marker data to detect genetic variants underlying local adaptation (Neale \& Ingvarsson, 2008).

Here we used resequencing data from 869 unrelated trees to understand the genetic mechanisms underlying local adaptation in P. trichocarpa. We take advantage of a common garden in Corvallis, OR to better understand the relative effects of environment and genotype on adaptive traits. Both climate and ecologically important traits vary extensively across the range of $P$. trichocarpa and some variation likely represents local adaptation (adaptation to climate). We built upon the previous population genomics study of $P$. trichocarpa (Evans et al., 2014) with a larger data set and sophisticated statistical methods. We used three conceptually different methods for testing for evidence of local adaptation. First, we tested for the correlation of phenotypic traits with climate variables of the source locations. Second, we used univariate, multivariate and PC-based GEA to identify loci potentially conferring local adaption. Third, we used RDA to answer three major questions: 1) What is the relative contribution of climate and geography in explaining neutral population genetic structure?; 2) What is the relative contribution of genomic, climate and geographic predictors in explaining the variation in adaptive traits?; and 3) Which predictor variables

significantly explain the variation in the genomic matrix and the adaptive trait matrix in the RDA models? Finally, we used this analysis to highlight outlier loci that potentially confer local adaptation in $P$. trichocarpa.

\subsection{Methods}

\subsubsection{Plantation establishment and sampling}

The $P$. trichocarpa association population consists of 1100 trees collected from natural populations in western Washington, Oregon, British Columbia, and California. The trees were clonally propagated from stem cuttings and planted in a common garden in Corvallis, Oregon in July of 2009 (Evans et al., 2014). The plantation consists of three blocks in a completely randomized design, and the trees were planted at $2 \mathrm{~m}$ x $3 \mathrm{~m}$ spacing. The plantation was coppiced in December of 2010 and again in December of 2013. Coppiced plants were allowed to re-sprout and grown for one season, after which they were pruned to a single leader in January of the following year (2012 and 2015, respectively). All 10 phenotypic traits [bud set, bud flush, carbon isotope, height, leaf area, leaf aspect ratio, petiole length and diameter, specific leaf area (SLA), chlorophyll content (SPAD) and stomatal density] reported here were measured 6 to 18 months after the second coppice except for bud set which was measured before December 2010. 
In June of 2014 leaf characteristics were measured for 1056 trees. The first and second fully expanded leaves (counting from the apex) were collected from a branch receiving full sunlight. One of the leaves was used for measuring petiole length and diameter with a digital caliper, and then scanned using a hand-held scanner. Images were analyzed to estimate leaf area, leaf length, leaf width, leaf perimeter, and leaf feret diameter using imageJ software (Schindelin, Rueden, Hiner, \& Eliceiri, 2015). Dry weights were determined for the same leaves for estimation of specific leaf area (SLA). Leaf chlorophyll was estimated using a SPAD 502 Plus meter (Spectrum Technologies) using the average of 3 replicate measures on different parts of the leaf. The other leaf was used for measuring abaxial stomatal density. The details for estimating stomatal density are described in Chhetri et al. (2019). In 2015, 2378 trees were measured for height. We measured bud set for 2169 trees (838 genotypes) in 2010 and bud flush for 2428 trees (869 genotypes) in 2013. Details of the phenotypic data collected from 2014 is described in Chhetri et al. (2019).

\subsubsection{Stable carbon isotope analysis}

In 2013, 759 trees were sampled for carbon isotope analysis. Wood cores were taken from breast height of the tree and the previous year's growth ring were selected for analysis. A cross section of the wood tissue representing the entire growth ring (about 1.2 to $1.8 \mathrm{mg}$ ) representing early, intermediate and late wood was sampled. Detailed methods for processing wood samples for carbon isotope analysis is described in Chhetri et al. (2019).

\subsubsection{Phenotypic data imputation}

We imputed missing phenotypic data using the phenix function of the phenix package in $\mathrm{R}$. Phenix imputes missing phenotypic data using a Bayesian matrix factorization model by accounting for the relatedness among the individuals (Dahl et al., 2016).

\subsubsection{Environmental data collection}

Climate and geography vary considerably across the range of distribution of $P$. trichocarpa (Supplementary Figure S3.1) The climate and geography data (26 variables, Tables $3.1 \& 3.2$ ) from the source location of 869 P. trichocarpa accessions from 1990 to 2010 were obtained from ClimateWNA (T. Wang, Hamann, Spittlehouse, \& Murdock, 2012). The average values across the years were used for all analyses.

\subsubsection{Correlation of adaptive traits with climate}

We first obtained genotypic best linear unbiased predictors of 11 phenotypic traits by running the mixed model analysis using the lmer function of the lme4 package in $\mathrm{R}$ and ran Pearson correlation of phenotypic traits with 26 climate variables and their first 4 PCs that explained more than $90 \%$ of the total variation cumulatively (Table 3.2). We also performed correlation of all phenotypic traits with geoclimate variables and their first four PCs (Table 3.3). 


\subsubsection{Test for the association of climate variables with the SNPs in the genome}

In order to identify the genetic variants that potentially drive local adaptation in $P$. trichocarpa we performed a genome-environment association (GEA) analysis using single and multirait and PC-based GEMMA (X. Zhou \& Stephens, 2012, 2014). The statistical test for association was performed for six climate variables - mean annual precipitation (MAP), mean annual temperature (MAT), mean warmest month temperature (MWMT), relative humidity $(\mathrm{RH})$, summer heat:moisture index (SHM) and continentality [TD, the difference between MWMT and mean coldest month temperature (MCMT)]. Pairwise correlations were run for all 26 climatic variables and only one variable from the group of highly correlated variables $(r>0.8)$ was selected for GWAS. The association test was also performed with the first two PCs of the 22 climate and 3 geography (latitude, longitude and elevation) variables that cumulatively explained more than $80 \%$ of the total variation. Furthermore, a multitrait association was performed for a set of all six selected climate variables. Because the mean annual radiation (MAR) dataset was incomplete, we removed it from the PC based GWAS.

The statistical test of association was performed with 869 re-sequenced unstructured individuals (Figure 3.1) using Genome-wide Efficient Mixed Model Association package (GEMMA, X. Zhou \& Stephens, 2012). The model tested was:

$y=W \alpha+x \beta+u+\epsilon$,

where $y$ is an $n$-vector of phenotypic BLUP values, where $n$ is the number of individuals tested; $W$ is an $n \times c$ matrix of covariates; $\alpha$ is a $c$-vector of corresponding coefficients, where $c$ is the number of principal coordinate axes used; $x$ is an $n$-vector of marker genotypes, $\beta$ is the effect size of the marker, $u$ is an n-vector of random effects that includes a relatedness matrix and $\epsilon$ is an $n$-vector of errors. A genetic relationship matrix estimated using GEMMA was used to control for the effects of relatedness in the association test. Phenotypic association was tested with 6.41 million SNPs that had MAF $>=0.05$. To correct for multiple testing, we used the Bonferroni correction method, but as this method is very stringent for a large number of tests, we relaxed our $P$-value cutoff from $7.417 \times 10^{-9}$ (Bonferroni correction threshold) to $P<1 \times 10^{-7}$, which we designated as a suggestive association threshold. Adjacent significant SNPs were aggregated into peaks using a 20kb moving window.

\subsubsection{Multivariate association: redundancy analysis}

Redundancy analysis (RDA) is a constrained ordination method that combines multiple linear regression and principal component analysis. It is an extension of multiple regression where the variation in a multivariate $\mathrm{Y}$ response matrix is explained by a multivariate $\mathrm{X}$ matrix of explanatory variables. RDA constructs a matrix $\mathrm{Y}^{\prime}$ of fitted genetic or phenotypic values estimated from the regression of each locus or phenotype and performs PCA on the matrix $\mathrm{Y}^{\prime}$ to produce canonical/constrained axes that are linear combinations of the predictors (Legendre \& Legendre, 2012; van den Wollenberg, 1977). Variables 
in the original response matrix are modeled as a function of predictor variables producing as many constrained axes as predictors. We performed redundancy analysis with two-fold objectives - first, in order to examine the effect of spatial and climate variation on the SNPs in the P. trichocarpa genome, and second, to test for association of multivariate phenotypic data with multivariate SNP, climate and geographical variables. SNP eigenvectors were generated using the program smartpca for the same $869 P$. trichocarpa accessions used for GEMMA association with 11.1 million SNPs after removing loci with MAF $<0.01$ (Patterson, Price, \& Reich, 2006). The 113 significant eigenvectors from smartpca were used for all RDA analyses.

For our first objective, we ran three RDA models - one with the SNP eigenvectors (EVs) as the response matrix constrained by the climate variables (model 1), second with SNP EVs as the response matrix constrained by the matrix of climate and spatial (geography or space) variables (model 2) and the other with the SNP EVs as the response matrix constrained by the climate variables with spatial variables used as a covariate (model 3). We used the 113 SNP eigenvectors as a response matrix and the five climate variables (MAP, MAT, MWMT, SHM and RH) out of the six variables selected for GEA above as the explanatory variables for model 1 . We removed TD from the RDA model because the variance inflation factor (VIF) for a few of the climate variables was greater than 10 when TD was included as one of the predictor variables, indicating multicollinearity. The same 5 climate variables selected for the climate RDA model 1 were used in the other two climate RDA models and a phenotypic RDA model (model 2, 3 and 4 below). We ran third degree polynomials on geographical variables (latitude and longitude) and selected the spatial variables (named as Space in the model) based on the stepwise regression (forward selection criteria) using ordistep function of the vegan package in R. We performed RDA analysis with the $r d a$ function of the vegan package (vegan 2.5-2) in R 3.5.1 (Oksanen et al., 2018; R Core Team, 2017).

SNP EVs $\sim$ Clim (RDA model 1)

SNP EVs $\sim$ Clim + Space (RDA model 2)

SNP EVs $\sim$ Clim + Condition (Space) (RDA model 3)

For our second objective, we ran the RDA model with a response matrix of 11 phenotypic traits constrained on the matrices of 113 significant SNP eigenvectors and 5 climate variables with the matrix of spatial variables as a covariate.

Pheno $\sim$ SNP EVs + Clim + Condition (Space) $($ RDA model 4)

For each of the significant (phenotypic RDA model) or outlier SNP EVs (climate RDA model) identified from each RDA model, we selected the top $0.1 \%$ SNPs from the tails of distribution of the SNP loadings (weightings) and considered those as the candidate for outlier loci. 


\subsection{Results}

\subsubsection{Trait heritabilities and genetic correlation of phenotypes with geography and climate variables}

Broad-sense heritabilities of the phenotypic traits showed the presence of substantial genetic variation. The heritability estimates ranged from 0.297 to 0.825 for all traits. Overall, the heritability estimates were higher for phenology traits $\left(H^{2}\right.$ for bud set and bud flush were 0.628 and 0.825 respectively) compared to morphological and physiological traits. Based on the pairwise correlation of geoclimate variables of the source locations of the $P$. trichocarpa accessions, in general, temperature, AHM, SHM and CMD decreased with latitude whereas TD and RH had positive correlation with latitude (Table 3.1). EMT, Eref, CMD and eFFP increased with temperature, whereas PAS decreased with temperature (Table 3.1). Bud set and bud flush had significant correlations with tree height and most of the other morphological traits (Table 3.3). While bud set and bud flush had significant negative correlation to each other, most traits including bud set, bud flush and tree height were strongly positively correlated with many climate and geographical variables, and PC1 of the 26 geo-climatic variables (Table $3.2 \& 3.3$ ). Bud flush and tree height had significant negative correlation with latitude whereas bud set had a significant positive correlation with latitude. Furthermore, tree height had a strong and significant correlation with temperature and MCMT and negative correlation with DD_0 and DD_18. Leaf area and SLA showed a similar trend as tree height - they had significant negative correlation with latitude and positive correlation with temperature. SLA and stomata had positive correlation with CMD, EXT and Eref whereas SPAD had negative correlation with MCMT. Leaf traits had moderate but significant correlations with selected climate and geographical variables, and PC1 of the 26 geo-climatic variables. Furthermore, stomatal density had significant negative correlation with relative humidity and positive correlation with EXT, Eref and CMD (Table 3.3).

\subsubsection{Detection of outlier loci using genotype-environment association (GEA)}

Using single and multitrait association of six climate variables and single trait association of PC1 and PC2 of 26 geoclimate variables with the 6.78 million SNPs, we identified a total of 422 significant SNPs (48 SNP peaks) in the vicinity of 66 genes based on a Bonferroni correction threshold of 5\% significance level $\left(P<7.417 \times 10^{-9}\right)$. Fourteen of these genes were shared across the three genotypeenvironment association (GEA) detection methods - single and multitrait and PC based associations (Figure 3.2a). Multitrait association detected the highest number of genes (48 genes, 99 significant SNPs) followed by single trait association (44 genes, 121 significant SNPs) and PC based single trait association (23 genes, 52 significant SNPs) (Figure 3.2a). With a relaxed $P$-value cutoff $\left(P<1 \times 10^{-7}\right), 833$ SNPs $(137$ SNP peaks) were detected, and these were near 172 genes (Figure 3.2b). 


\subsubsection{Genes identified by genotype-environment association (GEA)}

We identified some interesting genes across the genome for GEA with all (single, multitrait and PC based) GWAS methods. We found several genes in chromosome 10 for single trait (MAP, RH, SHM and TD), multitrait and PC2 GWAS (Figure 3.3; Supplemental Figure 3.2) including Potri.010G079500 and Potri.010G079600 code for a very long chain beta-ketoacyl-CoA synthase. The best match of this gene is KCS11 in Arabidopsis, which is potentially involved in cuticular wax biosynthesis. As expected, multitrait GWAS provided more power and detected genes with significant SNP peaks in several chromosomes (Figure 3.3), but single trait and PC based GWAS also detected genes with significant SNP peaks in various chromosomes (Table 3.4). For example, both PC1 and MAT GWAS revealed a strong peak near two genes: Potri.015G063400 (ABC transporter family protein) and Potri.015G063300 (bHLH family protein) on chromosome 15. Furthermore, PC2 and RH GWAS detected a gene, Potri.016G017400 that is related to the anthocyanin biosynthesis pathway.

\subsubsection{Redundancy analysis (RDA)}

\subsubsection{Association of genome-wide SNPs with geography and climate variables}

RDA analysis with the response matrix of 113 SNP EVs constrained on five climate variables (MAP, MAT, MWMT, SHM and RH) (model 1) showed that all climate variables significantly explained variation in the SNP EV response matrix. RDA model 2 with 113 SNP EVs constrained on five climate and 9 space variables showed that all predictor variables were significant. From RDA model 1, thirteen EVs (EVs 1 to 8, 10, 11, 14, 15 and 37) were identified as outliers from the tails (>3 standard deviations) of the distribution of EVs in each significant RDA axis. From RDA model 2, 17 EVs (all EVs identified in RDA model 1 plus EVs 18, 30, 32 and 33) were identified as outliers using the same criterion. Furthermore, there was a moderate population (river) level grouping of genotypes due to RDA axis 1 (Figure 3.4a \& b) and RDA axis 3 (Supplemental Figure S3.3a, b, c \& d) for both RDA model 1 and RDA model 2. Similarly, the partial RDA analysis with the 113 SNP EVs constrained on five climate variables conditioned on spatial variables (RDA model 3) showed that the explanatory matrix of climate variables significantly explained the variation in the response matrix of SNP eigenvectors. We identified five outlier SNP eigenvectors (EVs 2, 3, 5, 8 and 32) from the tails of the distribution of each significant RDA axis (Figure 3.5a; Supplemental Figure S3.5a-d). Compared to RDA model 1 and 2, there was no population level separation of genotypes due to RDA axis 1 and 3 in RDA model 3 (Figure 3.4c; Supplemental Figure S3.3e \& f). Comparing the three RDA models, among population genetic variation due to climate variables is confounded by geography (Figure 3.4a-c)

Partitioning the variation explained by constrained variables, climate explained only $2.6 \%$ whereas spatial variables (latitude and longitude) explained $5.6 \%$ of the variation in the constrained SNP 
eigenvectors, while both climate and geography shared less than $1 \%$ of the variation explained by the constrained variables (Figure 3.7a).

\subsubsection{Association of phenotypic traits with genome-wide SNPs, geography and climate variables}

Partial RDA analysis with the response matrix of 11 adaptive traits constrained on 113 SNP eigenvectors and five climatic variables conditioned on the matrix of spatial variables (RDA model 4) showed that the explanatory (predictor) variables significantly explained the variation in the phenotypic trait matrix. Out of all explanatory variables (113 SNP eigenvectors and 5 climate variables), 17 SNP eigenvectors significantly explained the variation in the phenotypes (Supplemental Figure S3.4). For the full phenotypic RDA model, (phenotypic matrix constrained on 113 SNP EVs, 5 climate variables and 7 space variables) we identified 16 SNP eigenvectors as significant. EVs 59 and 62 that were significant in the partial model were not significant in the full model, but an additional eigenvector EV61 was significant in full RDA model compared to the partial phenotypic RDA model.

Partitioning the variance explained by the constrained axis into the matrices of three explanatory variables, about $22 \%$ of the total variation in the RDA model was explained by the explanatory (constrained) variables that included 113 SNP eigenvectors, 5 climate variables and the matrix of spatial variables. SNP eigenvectors explained most of the variation with $21.6 \%$ while the other two predictor matrices, climate (5 variables) and the spatial matrix explained $12.3 \%$ and $16.6 \%$ of the total variation, respectively (Figure 3.7b).

\subsubsection{Comparison between Clim and Pheno RDA, and GEA}

A total of 7807 genes (48822 SNPs) were identified based on the selection of top $0.1 \%$ SNP loadings (weightings) from 5 significant SNP eigenvectors detected by the Clim RDA model (for e.g. EV32, Figure 3.5a). Comparing these genes with the genes from GEA analysis, 35 (based on Bonferroni cutoff of $7.417 \times 10^{-9}$ for GEA SNPs) and 79 genes (based on a suggestive association cutoff of $P<1 \times 10^{-7}$ ) were shared across the methods.

A total of 16782 genes (139988 SNPs) were identified based on selection of the top $0.1 \%$ SNP

loadings (weightings) from 17 significant SNP eigenvectors detected by the Pheno RDA model (for e.g. EV1, Figure 3.5b). Comparing these genes with the genes from GEMMA GEA analysis, 49 (based on Bonferroni cutoff of $P<7.417 \times 10^{-9}$ for GEA SNPs) and 118 genes (based on a suggestive association cutoff of $P<1 \times 10^{-7}$ ) were shared across the methods. All methods (Clim and Pheno RDA, and GEA) shared a total of 32 genes at $P<7.417 \times 10^{-9}$ for GEA SNPs (Table 3.4; Figure 3.6). 


\subsubsection{Comparing Pheno RDA model with simple multiple regression models}

With the phenotypic RDA model, we detected 17 significant SNP eigenvectors that significantly explained the variation in the matrix of 11 phenotypic traits (Figure 3.5b; Supplemental Figure S3.4, S3.6a-o). Comparing the simple multiple regression of each of the individual phenotypes in the RDA model with the phenotypic model, we found that 70 predictor variables including SNP eigenvectors, climate and space variables were significant in all simple multiple regression models combined. However, only 17 of them (all were SNP eigenvectors) were significant in the phenotypic RDA model. This difference is mainly driven by significant predictors from multiple regression for stomata, bud set and bud flush where most of the predictor variables that significantly explained the variation in the simple multiple regression were not significant in RDA model (Figure 3.8).

\subsection{Discussion}

Local adaptation is prevalent in forest trees such as Populus, despite large populations with weak neutral population structure (Aitken et al., 2008; Savolainen et al., 2013; Savolainen \& Pyhäjärvi, 2007). Local adaptation is particularly intriguing for wind pollinated trees like Populus where pollen can travel long distances (Slavov et al., 2009). Nevertheless, multiple studies have attempted to underscore the mechanisms of local adaptation in Populus (Capblancq et al., 2018; Evans et al., 2014; J. Wang et al., 2018). Here we attempted to provide the evidence for local adaption in P. trichocarpa by taking advantage of the phenotypic data collected in a common garden in Corvallis, OR and using combination of multiple data combinations (genotypic, phenotypic and geoclimatic data) and methods (RDA and GEA). We built upon the previous evidence of local adaption based on differentiation and GWAS methods (Evans et al., 2014; Chhetri et al., 2019) by incorporating larger genomic, phenotypic and geoclimatic data. We assessed the evidence of selection using the correlation of phenotypic traits with geoclimatic variables, identified outlier loci conferring local adaption using Genotype Environment Association (GEA) and used multivariate canonical redundancy analysis (RDA) to identify loci conferring local adaptation. We also used the latter method to partition the variation in neutral population structure into geography and climate predictor variables and the variation in phenotypic data into genomic, geographic and climatic predictor variables and highlighted their relative effects on local adaptation.

\subsubsection{Genetic variation (phenotypic trait) and phenotype environment correlation}

The presence of substantial heritable genetic variation in the phenotypic traits we studied here adds credibility to the genetic correlations of the phenotypic traits with the environmental variables and the multivariate regression of phenotypic traits with SNP PCs, climate and geography as predictor variables. Multiple studies have shown that climate is a driver of local adaption in Populus (Evans et al., 2014; Slavov \& Zhelev, 2010). Our results from this study suggest the same with significant genetic 
correlations of many of our phenotypic traits with climate of the source locations (Table 3.3). In congruence with other studies (Mckown et al., 2014), most of these traits and first and second PCs of the whole genome re-sequencing data have statistically significant correlations with latitude. Genetic correlations between the phenotypic traits and climate variables are likely confounded by geography and should be interpreted with caution (Chhetri et al., 2019).

However, for two reasons the confounding effects of geography on the relationships of phenotypic traits and the neutral genetic variation is less severe than previously thought in Populus. First, some phenotypes such as tree height, bud flush and bud set had much stronger correlations with environmental variables in our study compared to other studies (Mckown et al., 2014; Oubida et al., 2015). Second, SNP PC1 and SNP PC2 explain only 1.64 and $0.76 \%$ of the total SNP variation in our study (Supplementary Table S3.1) which is lower than previously reported (Mckown et al., 2014; Slavov et al., 2012), suggesting that our data reveal underlying patterns that were not previously detected. In support of this hypothesis, the correlations of SNP PC1 and SNP PC2 with latitude are not as strong as previously reported with smaller sample sizes (Mckown et al., 2014; Slavov et al., 2012).

Phenotypic traits significantly correlated with climate variables and climate PC axes are largely consistent with previous studies (Chhetri et al., 2019; Evans et al., 2014; Mckown et al., 2014). Bud flush was positively correlated, and bud set was negatively correlated with PC1 of 26 geo-climatic variables. PC1 of geoclimate variable is driven by the positive loadings of temperature related variables such as MAT, MCMT, DD5, DD18, MWMT, and moisture index related variables such as SHM and AHM and negative loadings of precipitation (MSP and MAP) related variables, latitude and elevation, and DD_18 and PAS (Table 3.2). These traits are quite important for growth, as early bud flush and late bud set were correlated with increased tree height.

Pairwise correlation of phenotypic traits and climate variables in our study suggest that taller trees generally have larger leaves, longer petioles, larger petiole diameter, but lower stomatal density in abaxial leaf surfaces. However, the contribution of stomatal patterning to tree growth is not clear as the wood carbon isotope composition that is indicative of abiotic stress tolerance showed no correlation with geoclimate as well as other phenotypic traits. Furthermore, there was no consistency across studies in the relationship of most physiological traits. Low trait heritabilities reported for most of these physiological traits makes it harder to make predictions on tree growth and stress tolerance in $P$. trichocarpa (Chhetri et al., 2019; Mckown et al., 2014).

\subsubsection{GEA outlier detection}

We found multiple genes with annotations consistent with their involvement in important plant functions on chromosome 10 and chromosome 16. For example, Potri.016G017400, is 255 bp upstream of a SNP that was associated with PC2 of 26 geoclimate variables $\left(P=3.75 \times 10^{-9}\right)$, as well as in the 
multitrait GWAS of 6 climate variables $\left(P=8.78 \times 10^{-8}\right)$. Potri.016G017400 encodes a putative glucosyl/glucuronosyl transferase, which is from the anthocyanin biosynthesis pathway. Anthocyanins are a group of secondary metabolites that have antioxidant properties. They play important roles in pigmentation and in defense against biotic and abiotic stresses. Genes involved in the anthocyanin biosynthesis pathway have been well characterized in the Brassicaceae family including Arabidopsis spp. and Brassica spp. (Guo et al., 2014; Misyura, Colasanti, \& Rothstein, 2013; Shi \& Xie, 2014) and the Solanaceace family including Solanum lycopersicum, Capsicum spp. and Solanum tuberosum (Liu et al., 2018). In Populus Potri.016G017400 is highly expressed in pre- and early dormant bud, spring and winter apical bud, and early male developmental processes (Figure 3.5).

On chromosome 10, Potri.010G079500 and Potri.010G080200 were close to major peaks in all GWAS analyses (single and multitrait, and PC-based methods). Two of these GWAS hits were within the coding regions of the genes - one was a non-synonymous SNP within the coding region of the Potri.010G079500 gene and the other was a synonymous SNP within the coding region of Potri.010G080200. Both of these genes are putative very long chain beta-ketoacyl-CoA synthase genes, which play a major role in the fatty acid biosynthesis pathway. Potri.010G079500 is highly expressed in young and first fully expanded leaves, late female development organs, stem treated with ammonia, nitrate and urea, female receptive organ, mid male developmental stage and apical and fully open bud. Potri.010G080200 is highly expressed in male and female early developmental stages, early and midspring apical buds, stem node and internode, young and immature leaves and root tips (Figure 3.6). The Potri.010G079500 gene also appears to be important for the alkene composition of cuticular wax in $P$. trichocarpa (Gonzales-Vigil et al., 2017). The gene is downregulated in trees with low alkene composition. Non-alkene producing trees had reduced growth and higher disease susceptibility, emphasizing the adaptive importance of this trait (Gonzales-Vigil et al., 2017).

\subsubsection{Outlier detection using RDA}

Because our SNP data set was large, we used an indirect approach for detecting the loci conferring local adaption. We used significant SNP PCs (eigenvectors) from the PCA analysis as a matrix of genetic data in the RDA analysis. Therefore, instead of identifying outlier loci directly from the RDA models, we identified outlier PCs that were potentially reflecting outlier SNP loadings (weightings) from the tails of the distribution. We extracted the top $0.1 \%$ of the SNPs from the tails of the distribution of the loadings and considered these as outlier loci. We identified 32 genes close to these outlier loci that were in common with the sets identified by the two RDA models and the GEA method. However, only one SNP PC was in common between the 5 and 17 outlier SNP PCs from the climate and phenotypic RDA models. The large number of genes we identified here (both for neutral population genetic structure and phenotypic traits) is not surprising considering that adaption in forest trees is highly polygenic, and the 
adaptive mechanism is different depending on the type of selective forces and the adaptive trait itself (Boyle, Li, \& Pritchard, 2017; Eckert et al., 2009; Evans et al., 2014; Holliday, Wang, \& Aitken, 2013). Under this scenario, selection can affect hundreds or thousands of loci such that the allele frequency of a given locus may not be altered much (Le Corre \& Kremer, 2012), thus requiring a powerful method like RDA to identify the presumed weak selection signal.

The RDA analysis for SNP, climate and geography matrices suggests that a very small portion of among-population variation (7.6\%) is explained by climate and geography in P. trichocarpa, out of which a large proportion of the variation is shared by both climate and geography variables. As climate alone explains only about $2.6 \%$ of the total variation, the large portion of variation in the SNP eigenvector matrix is due to population structure. A large proportion of the among population variance $(92.4 \%)$ remained unexplained. Part of the missing variance may be attributed to historical demographic processes or other environmental variables such as $\mathrm{pH}$ and soil characteristics not included in the RDA model. Nevertheless, all five of the climate variables in all Clim RDA models (RDA model 1, 2 and 3) significantly explained the variation in the SNP eigenvector response matrix.

Both climate and geography explained $12.3 \%$ and $16.6 \%$ of the variation respectively in the phenotypic matrix justifying the effect of climate in adaptive traits. This also matches with the significant correlations of some phenotypic traits with climate and geography (especially latitude) variables showing spatial clinal variation. Variance partitioning in the phenotypic RDA model showed that SNP data accounted for $21.664 \%$ of the phenotypic variation, although SNP data alone explained only $3.891 \%$ of the total phenotypic variation.

Simulation studies have shown that RDA has greater statistical power than GEA methods such as LFMM (Frichot et al., 2013), differentiation methods such as PCAdapt (a non-constrained ordination method, Luu, Bazin, and B. 2017), and other constrained ordination methods (Capblancq et al., 2018; Forester et al., 2018). Moreover, RDA-based methods have low false positive rates coupled with high true-positive rates compared to other general linear models (GLM) and LFMM methods and RDA is better than other methods in picking up weak selection signals (Forester et al., 2016, 2018). Given that adaptation is not always driven by strong differentiation at the individual loci and it is very likely that the covariance among key loci has a major role, small-effect single locus signatures can remain undetected from their neutral genomic background (Berg \& Coop, 2014; De Villemereuil et al., 2016; Le Corre \& Kremer, 2012; Lind et al., 2017; Rajora, Eckert, \& Zinck, 2016). Thus identification of adaptive loci is challenging and the use of multivariate statistics like RDA to unravel the complexity underlying adaptive genetic polymorphisms is warranted (Capblancq et al., 2018; De Kort et al., 2014; Forester et al., 2018; Lasky et al., 2012; Meirmans, 2012; Meirmans et al., 2017; Talbot et al., 2017; Vangestel et al., 2018). RDA methods identified outlier loci that were undetected in other genome scans (PCAdapt) and GEA 
(LFMM) methods in P. trichocarpa (Capblancq et al., 2018). Using a combination of trait-climate correlation, GEA and RDA methods we provided here a comprehensive assessment of the relative roles of geoclimatic, genomic and phenotype variation methods in understanding the genetic mechanisms underlying local adaption.

Local adaptation to varied environmental conditions allows trees to respond to the changing climate regime and can help in overcoming the potential adaptational lag. This study is an important step towards unraveling the evolutionary mechanisms in forest trees. Genomic signals identified here can have important implications in breeding programs and effective optimization of productivity traits for developing Populus as a biofuel feedstock. Furthermore, these findings can be invaluable for developing management and conservation strategies and sustainability of forest resources in the face of climate change.

\subsection{Conclusion}

Integrating genomic, environmental and phenotypic data we provided here a comprehensive assessment of the genetic basis of local adaption in P. trichocarpa. With the correlation of phenotypic traits with geography and climate variables we showed that the phenotypic traits have an environmental gradient that coincides in part with the latitudinal gradient. Using single trait, multitrait and PC-based GEA methods we detected adaptive signals that are common across all methods and identified key genes that potentially affect physiological functioning and adaptation in P. trichocarpa. We complemented our GEA analysis with a conceptually different multivariate ordination method, RDA, where we identified genomic outliers (genes) from both phenotypic and climate RDA models that overlapped with the genes identified from the GEA methods. Variance partitioning of the response matrices in both climate and phenotypic models underscores the importance of geographical variation in neutral population genetic structure (climate RDA model) and adaptive genetic variation (phenotypic RDA model). These findings have important implications for effective optimization of productivity traits, and developing sustainable management and conservation strategies of forest trees. Given that local adaption is believed to be under polygenic control, outlier loci detection methods should incorporate multivariate methods that allow for simultaneous detection of adaptive genomic signals covarying with the adaptive forces, or at least the univariate methods should be complemented with multivariate methods. Furthermore, to gain better insights into the adaption of tree populations it is important to integrate genotypic, environmental and phenotypic data whenever possible. Nevertheless, given the polygenic nature of the adaption we need larger sample size from across the range of distribution range of tree species at the whole genome level to accurately estimate the genome-wide signals contributing to local adaption. 


\subsection{Tables and figures}

Table 3.1 Pearson correlation (r) between 26 geo-climate variables and PCs $1-4$ of 26 geo-climate variables of the source location of $P$.

trichocarpa genotypes. Values greater than 0.13 or less than -0.13 are significant based on the Bonferroni correction criteria at $5 \%$ significance level.

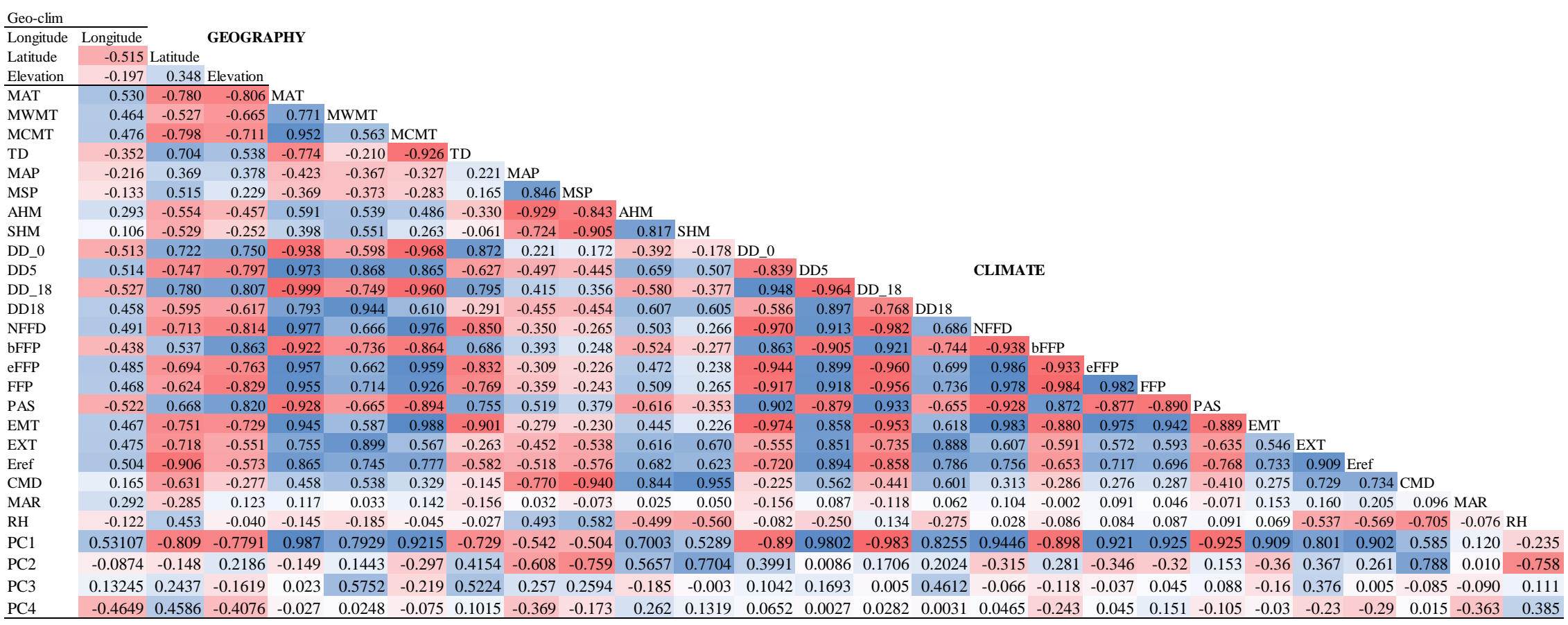


Table 3.2 PCA loadings of 26 geo-climate variables for the first 4 PCs. PC1, PC2, PC3 and PC4 explained $62.44 \%, 17.13 \%, 6.02 \%$ and $5.20 \%$ of the total variation, respectively. Dark blue and dark red colors indicate high positive and high negative values, respectively.

\begin{tabular}{|c|c|c|c|c|c|}
\hline Geo-climate variable & Abbreviation & PC1 & $\mathrm{PC} 2$ & PC3 & $\mathrm{PC} 4$ \\
\hline Mean Annual Temperature $\left({ }^{\circ} \mathrm{C}\right)$ & MAT & 0.245 & -0.071 & 0.001 & -0.023 \\
\hline Degree-days Above $5^{\circ} \mathrm{C}$ & DD5 & 0.243 & 0.003 & -0.070 & -0.125 \\
\hline Number of Frost-Free Days & NFFD & 0.234 & -0.150 & -0.011 & 0.067 \\
\hline Frost Free Period & FFP & 0.229 & -0.151 & -0.115 & 0.016 \\
\hline Mean Coldest Month Temperature $\left({ }^{\circ} \mathrm{C}\right)$ & MCMT & 0.229 & -0.141 & 0.109 & 0.148 \\
\hline Date of End of FFP & eFFP & 0.228 & -0.165 & -0.020 & 0.044 \\
\hline Extreme Minimum Temperature of 30 Years & EMT & 0.226 & -0.171 & 0.074 & 0.109 \\
\hline Hargreaves Reference Evaporation & Eref & 0.225 & 0.124 & 0.166 & -0.092 \\
\hline Degree-days Above $18^{\circ} \mathrm{C}$ & DD18 & 0.206 & 0.095 & -0.151 & -0.356 \\
\hline Extreme Maximum Temperature of 30 Years & EXT & 0.200 & 0.174 & 0.018 & -0.367 \\
\hline Mean Warmest Month Temperature $\left({ }^{\circ} \mathrm{C}\right)$ & MWMT & 0.197 & 0.067 & -0.202 & -0.441 \\
\hline Annual Heat:Moisture Index & AHM & 0.175 & 0.269 & -0.102 & 0.234 \\
\hline Hargreaves Climatic Moisture Deficit & CMD & 0.146 & 0.374 & 0.019 & 0.064 \\
\hline Longitude & Long & 0.133 & -0.043 & 0.306 & -0.263 \\
\hline Summer Heat:Moisture Index & SHM & 0.132 & 0.367 & -0.069 & 0.027 \\
\hline Mean annual radiation & MAR & 0.032 & 0.006 & 0.586 & -0.147 \\
\hline Mean annual relative humidity & RH & -0.059 & -0.363 & -0.215 & 0.009 \\
\hline Mean Annual Summer Precipitation (mm) & MSP & -0.126 & -0.362 & 0.006 & -0.244 \\
\hline Mean Annual Precipitation (mm) & MAP & -0.135 & -0.287 & 0.149 & -0.331 \\
\hline Continentality (difference between MWMT and MCMT) & TD & -0.181 & 0.197 & -0.223 & -0.378 \\
\hline Elevation $(\mathrm{m})$ & Elev & -0.193 & 0.104 & 0.349 & -0.017 \\
\hline Latitude & Lat & -0.202 & -0.071 & -0.365 & -0.037 \\
\hline Degree-days Below $0^{\circ} \mathrm{C}$ & DD_0 & -0.221 & 0.189 & -0.081 & -0.056 \\
\hline Date of Beginning of FFP & bFFP & -0.222 & 0.135 & 0.198 & 0.010 \\
\hline Precipitation as Snow (mm) & PAS & -0.230 & 0.073 & 0.045 & -0.108 \\
\hline Degree-days below $18^{\circ} \mathrm{C}$ & DD_18 & -0.244 & 0.081 & -0.009 & 0.001 \\
\hline
\end{tabular}


Table 3.3 Pearson correlation between phenotypic traits and geo-climate variables and PCs of 26 geoclimate variables. Values greater than 0.12 or less than -0.12 are significant at the $5 \%$ Bonferroni correction level of significance. Dark blue and dark red colors indicate high positive and high negative $P$ values, respectively.

\begin{tabular}{|c|c|c|c|c|c|c|c|c|c|c|c|}
\hline $\begin{array}{l}\text { Geo- } \\
\text { climate*/Trait }\end{array}$ & Bud flush & Bud set & Height & Leaf area & $\begin{array}{l}\begin{array}{l}\text { Petiole } \\
\text { length }\end{array} \\
\end{array}$ & $\begin{array}{l}\begin{array}{l}\text { Petiole } \\
\text { diameter }\end{array} \\
\end{array}$ & $\begin{array}{l}\text { Leaf aspect } \\
\text { ratio }\end{array}$ & $\begin{array}{l}\text { Specific } \\
\text { leaf area } \\
\end{array}$ & $\begin{array}{l}\text { Stomatal } \\
\text { density } \\
\end{array}$ & $\begin{array}{l}\text { Carbon } \\
\text { isotope } \\
\end{array}$ & SPAD \\
\hline Long & 0.374 & -0.435 & 0.427 & 0.343 & 0.309 & 0.233 & -0.165 & 0.012 & -0.088 & 0.031 & -0.017 \\
\hline Lat & -0.508 & 0.61 & -0.418 & -0.149 & -0.179 & -0.096 & 0.212 & -0.329 & -0.17 & 0.026 & 0.175 \\
\hline Elev & -0.256 & 0.293 & -0.229 & -0.072 & -0.149 & -0.033 & 0.123 & -0.131 & -0.035 & 0.12 & 0.091 \\
\hline MAT & 0.456 & -0.555 & 0.431 & 0.194 & 0.238 & 0.115 & -0.224 & 0.223 & 0.07 & -0.085 & -0.155 \\
\hline MWMT & 0.358 & -0.301 & 0.21 & 0.078 & 0.093 & 0.069 & -0.151 & 0.082 & 0.093 & -0.023 & -0.086 \\
\hline MCMT & 0.418 & -0.587 & 0.471 & 0.221 & 0.26 & 0.126 & -0.236 & 0.25 & 0.039 & -0.082 & -0.17 \\
\hline $\mathrm{TD}$ & -0.332 & 0.556 & -0.462 & -0.225 & -0.264 & -0.117 & 0.209 & -0.256 & -0.004 & 0.089 & 0.161 \\
\hline MAP & -0.339 & 0.252 & -0.192 & -0.014 & -0.058 & -0.023 & 0.025 & -0.137 & -0.031 & -0.035 & -0.008 \\
\hline MSP & -0.314 & 0.285 & -0.174 & 0.067 & 0.01 & 0.036 & 0.02 & -0.222 & -0.117 & -0.057 & 0.036 \\
\hline AHM & 0.413 & -0.373 & 0.248 & 0.04 & 0.081 & 0.04 & -0.074 & 0.19 & 0.075 & -0.006 & -0.05 \\
\hline SHM & 0.317 & -0.247 & 0.101 & -0.086 & -0.052 & -0.046 & -0.034 & 0.197 & 0.137 & 0.039 & -0.053 \\
\hline DD_0 & -0.351 & 0.556 & -0.457 & -0.239 & -0.273 & -0.138 & 0.232 & -0.219 & -0.018 & 0.072 & 0.154 \\
\hline DD5 & 0.478 & -0.5 & 0.372 & 0.151 & 0.196 & 0.094 & -0.203 & 0.197 & 0.098 & -0.084 & -0.143 \\
\hline DD_18 & -0.452 & 0.56 & -0.436 & -0.2 & -0.243 & -0.117 & 0.224 & -0.226 & -0.065 & 0.087 & 0.156 \\
\hline DD18 & 0.41 & -0.342 & 0.234 & 0.073 & 0.107 & 0.061 & -0.16 & 0.106 & 0.113 & -0.044 & -0.109 \\
\hline NFFD & 0.397 & -0.548 & 0.444 & 0.218 & 0.256 & 0.126 & -0.23 & 0.211 & 0.022 & -0.086 & -0.152 \\
\hline bFFP & -0.37 & 0.431 & -0.367 & -0.184 & -0.218 & -0.117 & 0.208 & -0.128 & 0.017 & 0.095 & 0.116 \\
\hline eFFP & 0.386 & -0.541 & 0.429 & 0.221 & 0.253 & 0.134 & -0.234 & 0.187 & 0.011 & -0.082 & -0.149 \\
\hline FFP & 0.385 & -0.492 & 0.403 & 0.205 & 0.238 & 0.127 & -0.225 & 0.159 & -0.003 & -0.09 & -0.134 \\
\hline PAS & -0.388 & 0.506 & -0.424 & -0.208 & -0.243 & -0.121 & 0.176 & -0.188 & 0.002 & 0.042 & 0.1 \\
\hline EMT & 0.374 & -0.562 & 0.453 & 0.22 & 0.254 & 0.126 & -0.242 & 0.233 & 0.029 & -0.066 & -0.166 \\
\hline EXT & 0.458 & -0.373 & 0.239 & 0.051 & 0.094 & 0.037 & -0.143 & 0.201 & 0.211 & -0.038 & -0.127 \\
\hline Eref & 0.537 & -0.535 & 0.366 & 0.12 & 0.164 & 0.073 & -0.178 & 0.283 & 0.185 & -0.059 & -0.157 \\
\hline CMD & 0.393 & -0.311 & 0.154 & -0.069 & -0.02 & -0.035 & -0.04 & 0.238 & 0.179 & 0.023 & -0.068 \\
\hline MAR & 0.112 & -0.201 & 0.163 & 0.101 & 0.131 & 0.038 & -0.066 & 0.114 & 0.056 & 0.032 & -0.055 \\
\hline RH & -0.37 & 0.15 & -0.054 & 0.073 & 0.033 & 0.052 & -0.024 & -0.174 & -0.226 & 0.045 & 0.049 \\
\hline $\mathrm{PC} 1$ & 0.483 & -0.559 & 0.424 & 0.174 & 0.219 & 0.107 & -0.212 & 0.238 & 0.081 & -0.066 & -0.147 \\
\hline PC2 & 0.163 & 0.048 & -0.121 & -0.184 & -0.165 & -0.102 & 0.101 & 0.085 & 0.158 & 0.071 & 0.038 \\
\hline PC3 & -0.049 & 0.232 & -0.188 & -0.05 & -0.073 & -0.008 & 0.011 & -0.2 & 0.028 & -0.01 & 0.035 \\
\hline $\mathrm{PC} 4$ & -0.206 & 0.213 & -0.163 & -0.138 & -0.102 & -0.085 & 0.083 & -0.084 & -0.117 & -0.006 & 0.076 \\
\hline Bud flush & NA & -0.340 & 0.312 & 0.190 & 0.190 & 0.145 & -0.142 & 0.187 & 0.067 & 0.036 & -0.070 \\
\hline Bud set & NA & NA & -0.514 & -0.287 & -0.304 & -0.201 & 0.217 & -0.266 & -0.061 & 0.097 & 0.134 \\
\hline
\end{tabular}


Table 3.4 Genes identified from P. trichocarpa single and multitrait GWAS shared by climate and phenotype RDA models.

\begin{tabular}{|c|c|c|c|}
\hline Gene model ${ }^{\mathrm{a}}$ & Trait $^{\mathrm{b}}$ & $P$-value ${ }^{\mathrm{c}}$ & Functional annotation \\
\hline Potri.004G163400 & RH & $2.25 \mathrm{E}-10$ & $\begin{array}{l}\text { similar to Vacuolar ATP synthase } 16 \mathrm{kDa} \text { proteolipid subunit } \\
1 / 3 / 5\end{array}$ \\
\hline Potri.005G095500 & TD & 7.38E-13 & $\begin{array}{l}\text { similar to unnamed protein product (Fragaria } \mathrm{x} \text { ananassa, co- } \\
\text { ortholog of several CAD genes }\end{array}$ \\
\hline Potri.006G082100 & multitrait* & $3.62 \mathrm{E}-10$ & $\begin{array}{l}\text { similar to expressed protein in Arabidopsis thaliana; similar to } \\
\text { expressed protein in Arabidopsis thaliana; [ortholog of } \\
\text { At3g53670,At2g37480,] }\end{array}$ \\
\hline Potri.006G082200 & multitrait & $6.39 \mathrm{E}-09$ & NA \\
\hline Potri.006G170700 & multitrait & $1.09 \mathrm{E}-10$ & $\begin{array}{l}\text { PTHR23257:SF331 - OCTICOSAPEPTIDE/PHOX/BEM1P } \\
\text { DOMAIN-CONTAINING PROTEIN KINASE }\end{array}$ \\
\hline Potri.006G171200 & multitrait & $7.98 \mathrm{E}-10$ & $\begin{array}{l}\text { PTHR11926//PTHR11926:SF221 - } \\
\text { GLUCOSYL/GLUCURONOSYL TRANSFERASES }\end{array}$ \\
\hline Potri.008G131700 & multitrait & $1.58 \mathrm{E}-09$ & $\begin{array}{l}\text { ORG:Malus x domestica, co-ortholog of AAQ96165, } \\
\text { AAQ96164, At1g14920, At2g01570, AAQ54509 }\end{array}$ \\
\hline Potri.008G210400 & TD & 4.87E-09 & NA \\
\hline Potri.008G210500 & TD & $3.41 \mathrm{E}-10$ & NA \\
\hline Potri.008G210600 & $\mathrm{TD}$ & $1.42 \mathrm{E}-09$ & NA \\
\hline Potri.010G079300 & $\mathrm{PC} 2^{* *}$ & $2.34 \mathrm{E}-10$ & NA \\
\hline Potri.010G079400 & $\mathrm{PC} 2$ & $1.47 \mathrm{E}-11$ & NA \\
\hline Potri.010G079500 & multitrait & 4.30E-30 & NA \\
\hline Potri.010G079600 & multitrait & $6.80 \mathrm{E}-29$ & PTHR13288 - DNA-DAMAGE REPAIR PROTEIN DRT111 \\
\hline Potri.010G079700 & PC2 & $6.48 \mathrm{E}-20$ & NA \\
\hline Potri.010G079800 & $\mathrm{TD}$ & $1.56 \mathrm{E}-09$ & NA \\
\hline Potri.010G080000 & multitrait & $4.88 \mathrm{E}-38$ & NA \\
\hline Potri.010G080100 & $\mathrm{TD}$ & $8.84 \mathrm{E}-11$ & NA \\
\hline
\end{tabular}




\begin{tabular}{|c|c|c|c|}
\hline Gene model ${ }^{\mathrm{a}}$ & Trait $^{\mathrm{b}}$ & $P$-value ${ }^{\mathrm{c}}$ & Functional annotation \\
\hline Potri.010G080200 & multitrait & $4.09 \mathrm{E}-42$ & NA \\
\hline Potri.010G080300 & multitrait & $6.24 \mathrm{E}-19$ & $\begin{array}{l}\text { PTHR22773:SF88 - NADH-UBIQUINONE } \\
\text { OXIDOREDUCTASE CHAIN } 4\end{array}$ \\
\hline Potri.010G080400 & multitrait & $5.78 \mathrm{E}-24$ & NA \\
\hline Potri.010G080500 & multitrait & $1.79 \mathrm{E}-16$ & NA \\
\hline Potri.010G080600 & multitrait & $5.12 \mathrm{E}-12$ & $\begin{array}{l}\text { PF04720 - PDDEXK-like family of unknown function } \\
\text { (PDDEXK_6) }\end{array}$ \\
\hline Potri.014G104800 & MWMT & $8.71 \mathrm{E}-11$ & $\begin{array}{l}\text { no apical meristem (NAM) family protein; similar to no apical } \\
\text { meristem (NAM) - Petunia hybrida; similar to }\end{array}$ \\
\hline Potri.014G106400 & MWMT & $1.36 \mathrm{E}-09$ & $\begin{array}{l}\text { EMBL:PHDNANAM (co-ortholog of At } 3 \mathrm{~g} 61910, \text { At } 2 \mathrm{~g} 46770 \text { ) } \\
\text { similar to Thylakoid membrane phosphoprotein } 14 \mathrm{kDa} ; \\
\text { similar to chloroplast precursor }\end{array}$ \\
\hline Potri.015G016700 & RH & $1.93 \mathrm{E}-09$ & PF02362 - B3 DNA binding domain (B3) \\
\hline Potri.016G011500 & multitrait & 7.81E-14 & Transporting two-sector ATPase / Mitochondrial ATPase \\
\hline Potri.016G017400 & $\mathrm{PC} 2$ & $3.75 \mathrm{E}-09$ & $\begin{array}{l}\text { PTHR11926//PTHR11926:SF267 - } \\
\text { GLUCOSYL/GLUCURONOSYL TRANSFERASES }\end{array}$ \\
\hline Potri.016G090400 & multitrait & $1.46 \mathrm{E}-11$ & $\begin{array}{l}\text { PF00651//PF03000 - BTB/POZ domain (BTB) // NPH3 family } \\
\text { (NPH3) }\end{array}$ \\
\hline Potri.016G095100 & PC2 & $6.55 \mathrm{E}-11$ & $\begin{array}{l}\text { PTHR23180:SF293 - ADP-RIBOSYLATION FACTOR } \\
\text { GTPASE-ACTIVATING PROTEIN AGD7 }\end{array}$ \\
\hline Potri.017G143000 & multitrait & 2.14E-09 & $\begin{array}{l}\text { protein kinase family protein; similar to protein kinase domain } \\
\text { containing protein (co-ortholog of At5g24010) }\end{array}$ \\
\hline Potri.018G055100 & multitrait & 1.69E-09 & $\begin{array}{l}\text { PTHR32263:SF8 - INACTIVE POLY [ADP-RIBOSE] } \\
\text { POLYMERASE SRO2-RELATED }\end{array}$ \\
\hline
\end{tabular}

${ }^{\mathrm{a}}$ Gene models are annotated using v3 of the $P$. trichocarpa genome

${ }^{\mathrm{b}}$ Full trait names provide in Table 2

${ }^{\mathrm{c}} \mathrm{SNP} \mathrm{p}$ values $<1 \times 10^{-9}$

*Multitrait set that includes MAP, MAT, MWMT, RH, SHM and TD climate variables

**PC2 of all 26 geoclimate variables listed in Table 2 


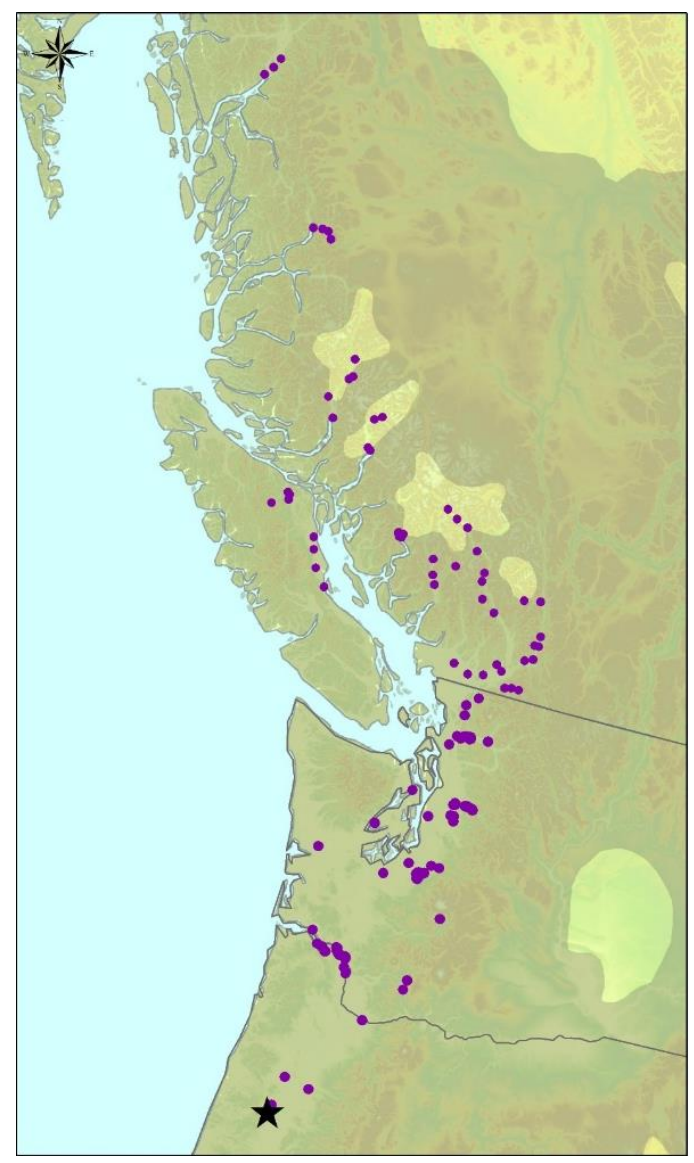

(a)

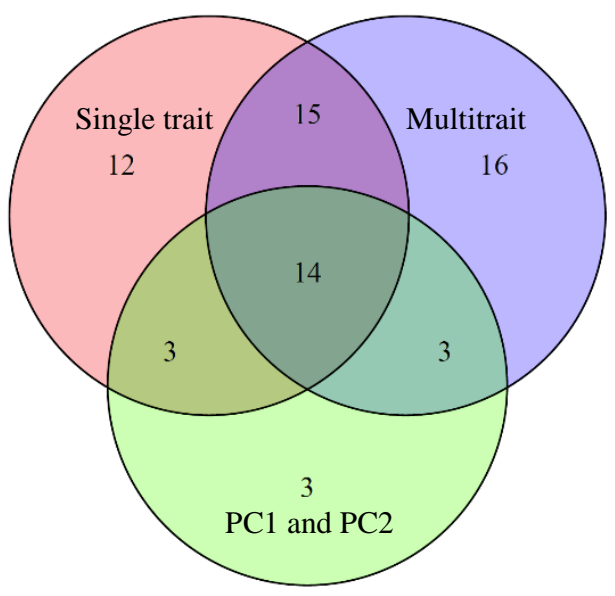

Figure 3.1 Source locations of $869 P$.

trichocarpa genotypes sampled in this study (purple dots). The trees were grown in a common garden in Corvallis, Oregon (black star).

(b)

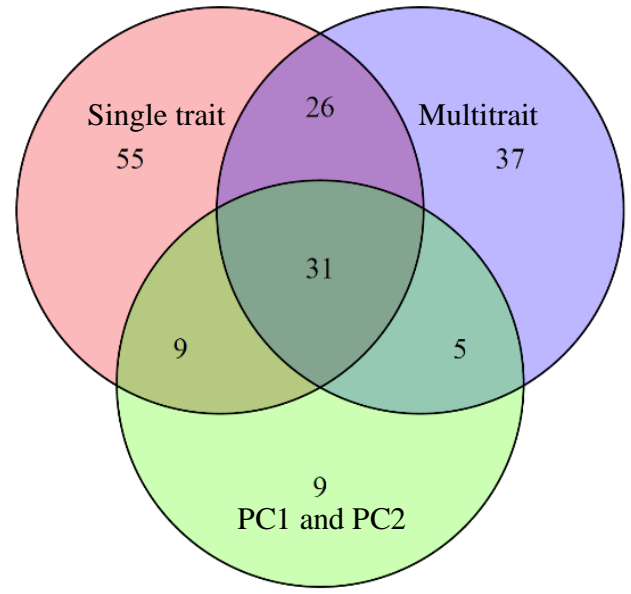

Figure 3.2 Genes detected by genotype environment association (GEA) across the methods - single trait, multitrait and PC-based. (a) $P<7.417 \times 10^{-9}$ (Bonferroni correction threshold). (b) $P<1 \times 10^{-7}$. 

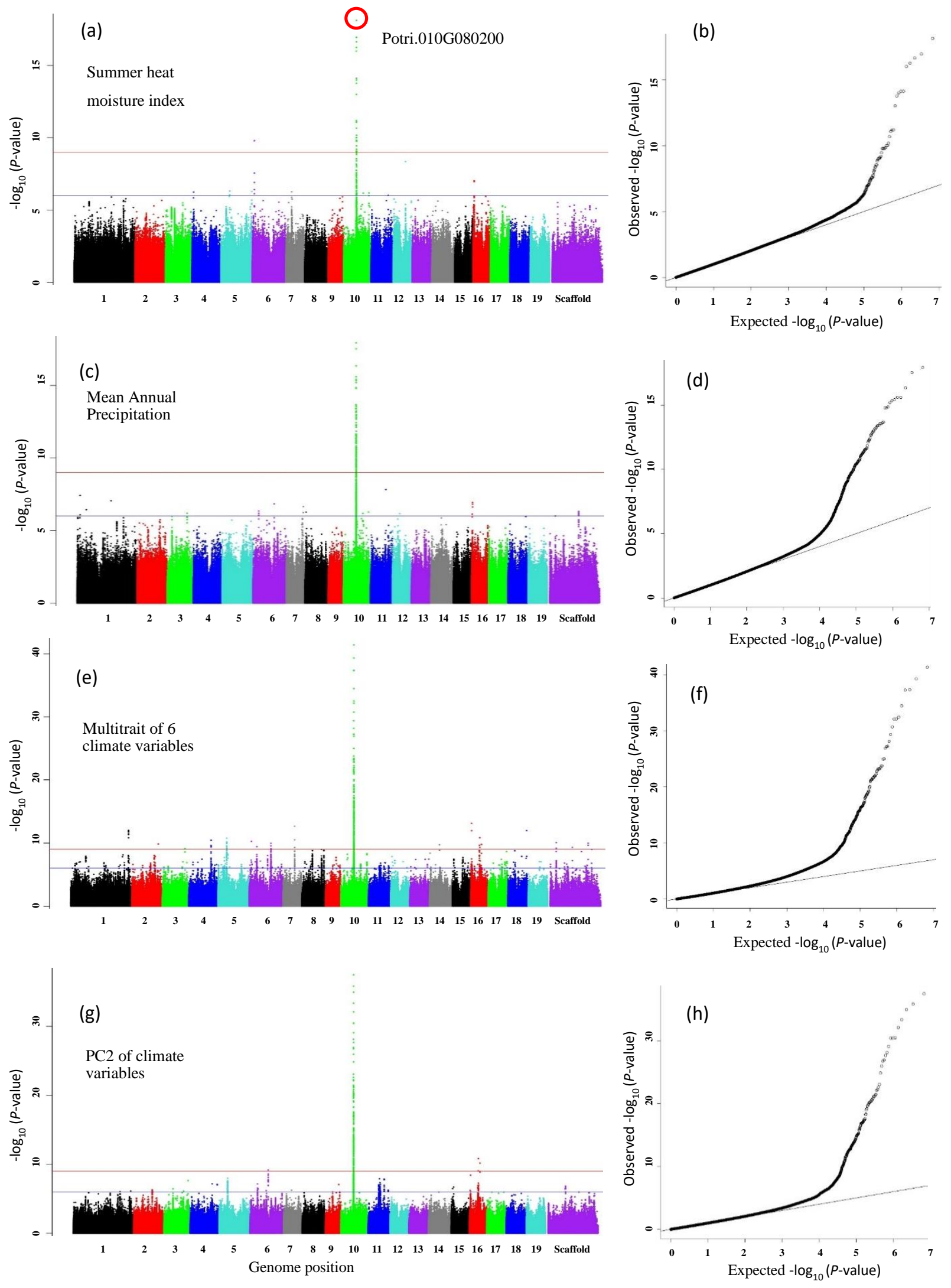
Figure 3.3 Single trait, multitrait and PC based association showing the association of geo-climate variables with the SNPs in chromosome 10 - Manhattan (left) and QQ plots (right). Numbers 1 to 19 represent chromosomes; scaffolds are the reads that did not align to any of the 19 chromosomes. (a) and (b) Summer heat:moisture index (SHM) (c) and (d) Mean annual precipitation (MAP) (e) and (f) Multitrait GWAS of six climate variables - MAP, MAT (mean annual temperature), MWMT (mean warmest month temperature), RH (relative humidity), SHM and TD [temperature difference between MWMT and MCMT (mean coldest month temperature)]. (g) and (h) PC2 of 25 geo-climate variables (includes latitude, longitude and elevation, and 22 climate variables). Red horizontal line indicates Bonferroni correction threshold $\left(P=7.417 \times 10^{-9}\right)$ and blue horizontal line indicates suggestive association threshold $\left(P=1 \times 10^{-7}\right)$. Nearest gene to the highlighted SNP/s (red circle) is Potri.010G080200, which is a very long chain beta-ketoacyl-CoA synthase with potential involvement in cuticular wax biosynthesis.

(a)

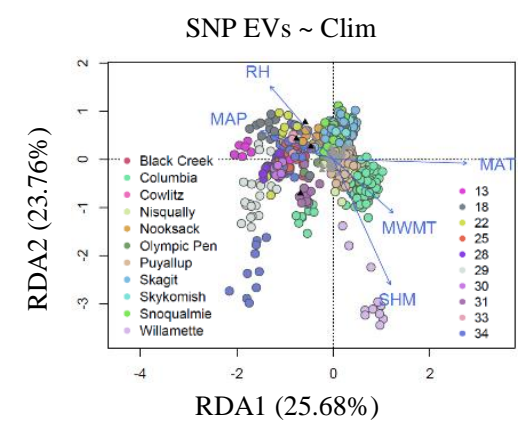

(b)

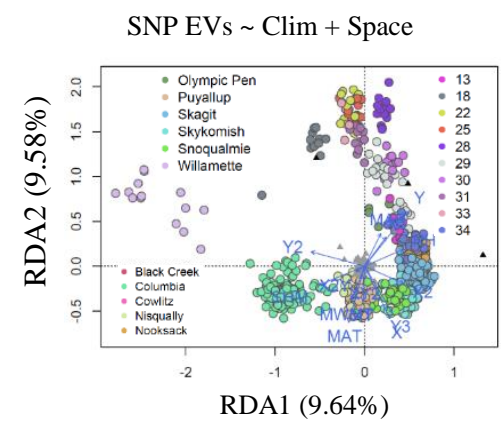

(c)

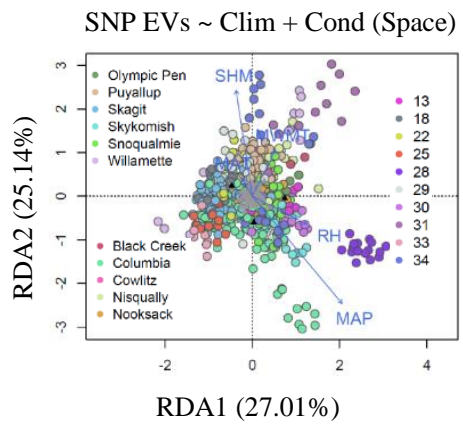

Figure 3.4 Triplots showing RDA on SNP eigenvectors constrained by the matrix of climate variables (a), climate and space variables (b) and climate variables with space as covariates (c). Both RDA1 and RDA2 axes were significant in all RDA models. It appears that the separation of $P$. trichocarpa populations in the RDA axis 1 in the climate only RDA model is due to the confounding effect of spatial variation. Circles indicate 869 P. trichocarpa genotypes (color coded by populations, the rivers). Triangles indicate SNP eigenvectors with outlier eigenvectors (based on a 3 standard deviation cutoff selected from the tails of the distribution of RDA axes) in black. Blue arrows indicate the influence of climate variables on RDA axis. 

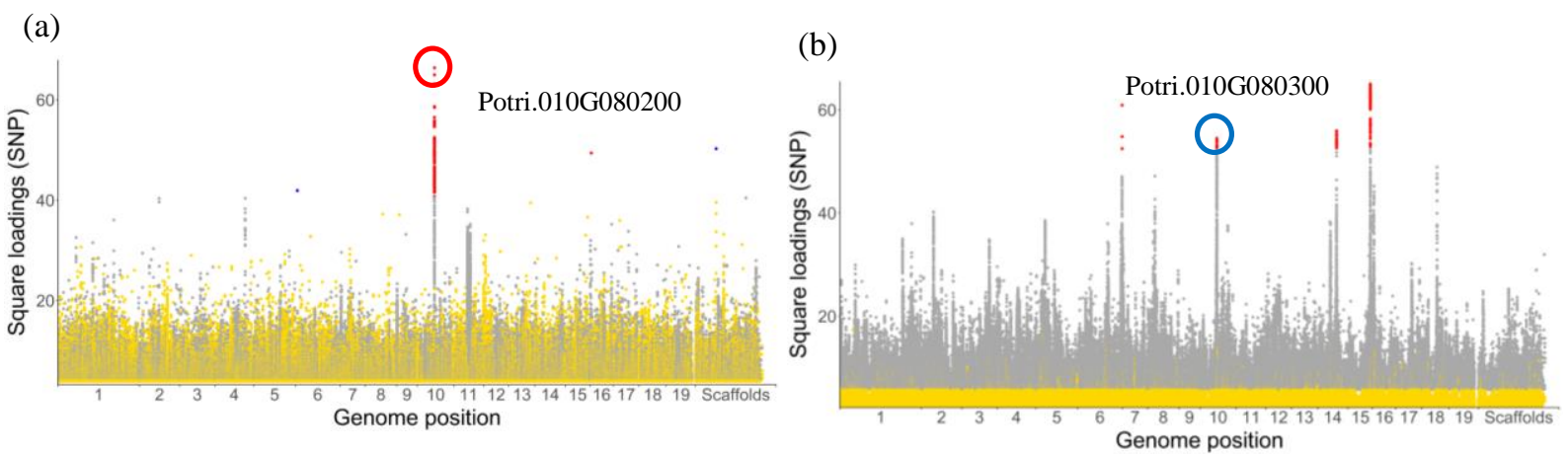

Figure 3.5 Square loadings of top $0.1 \%$ SNPs from one of the 5 significant eigenvectors (EV32) detected from the climate RDA analysis (a) and one of the 17 outlier eigenvectors (EV1) detected from the phenotypic RDA analysis (b). Numbers 1 to 19 represent chromosomes; scaffolds are the reads that did not align to any of the 19 chromosomes. Gray and yellow colors represent positive and negative square loadings, respectively. Red and blue circles indicate top 100 square loadings corresponding to positive and negative loadings, respectively. Climate RDA (EV32) identified the same gene (circled red) that was identified in GEA. Phenotypic RDA (EV1) also identified a similar gene, Potri.010G80300 (circled blue) just 100 bp upstream of the Potri.01G080200. 

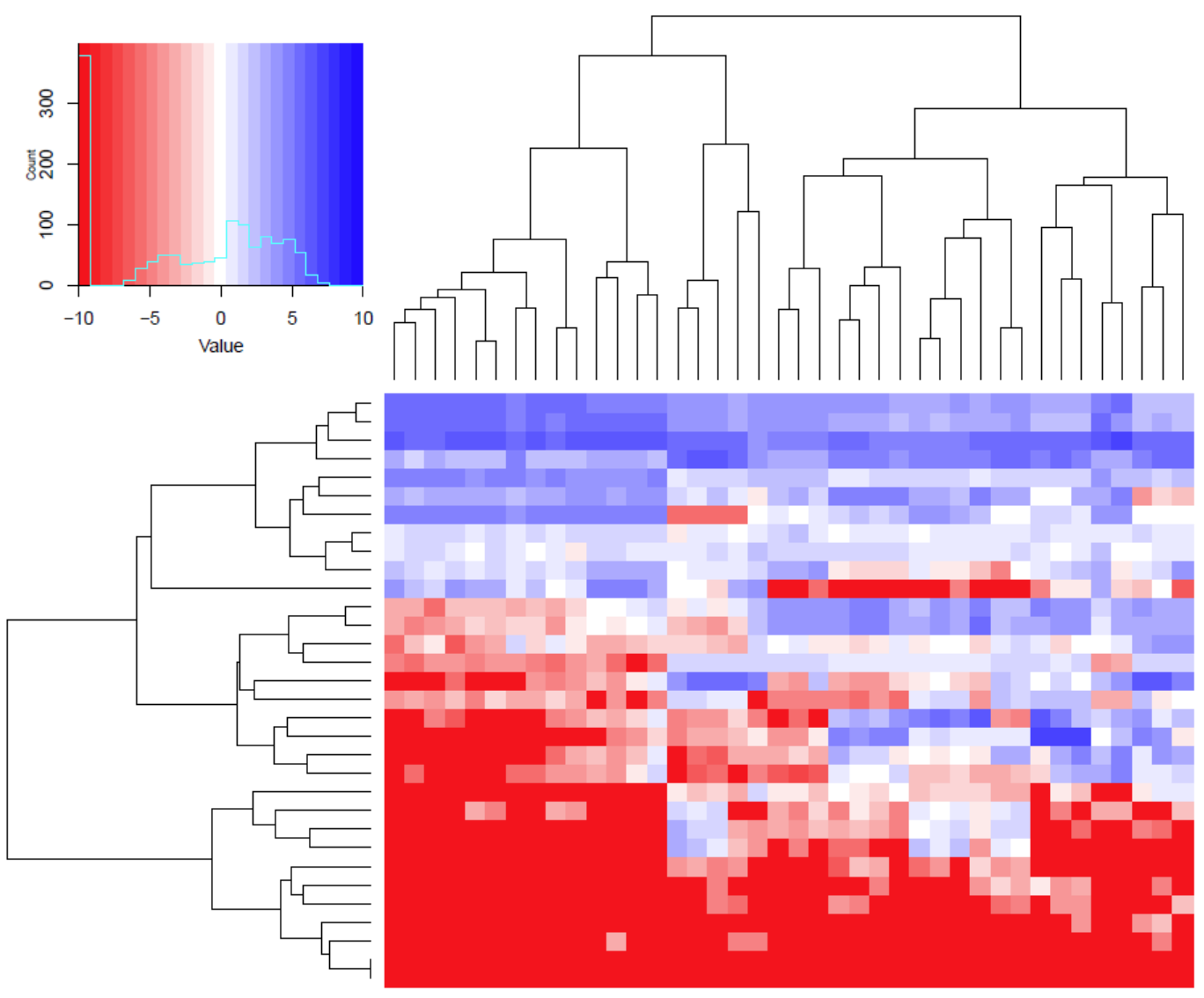

Potri.008G131700

Potri.004G163400

Potri.010G080600

Potri.006G170700

Potri.006G171200

Potri.014G106400

Potri.018G055100

Potri.016G017400

Potri.005G095500

Potri.010G079300

Potri.010G079400

Potri.016G090400

Potri.016G095100

Potri.010G080400

Potri.014G104800

Potri.010G079500

Potri. 10

Potri.010G080200

Potri.010 G080000

Potri.010G080100

Potri.008G210400

Potri.008 G210600

Potri.010G080300

Potri.015G016700

Potri.016G011500

Potri.010G07900

Potri.010G079600

Potri 010G080500

Potri.006G082200

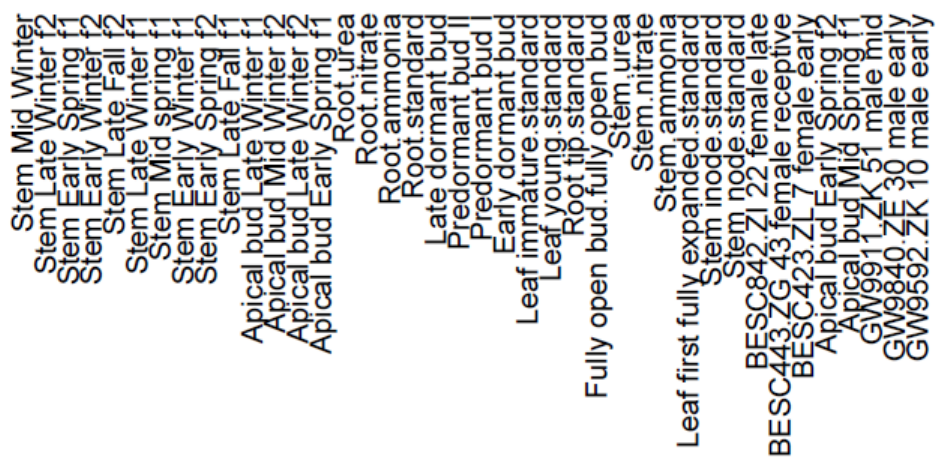

Figure 3.6 Expression level of genes identified from single and multitrait GWAS shared by climate and phenotype RDA models in different tissues in P. trichocarpa. 
(a)

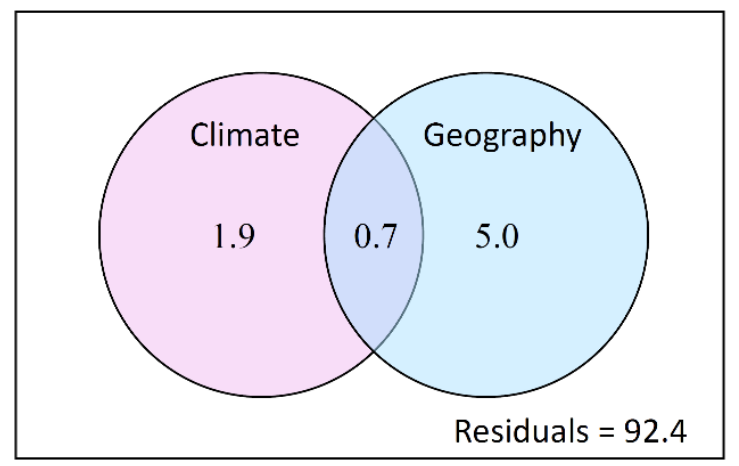

(b)

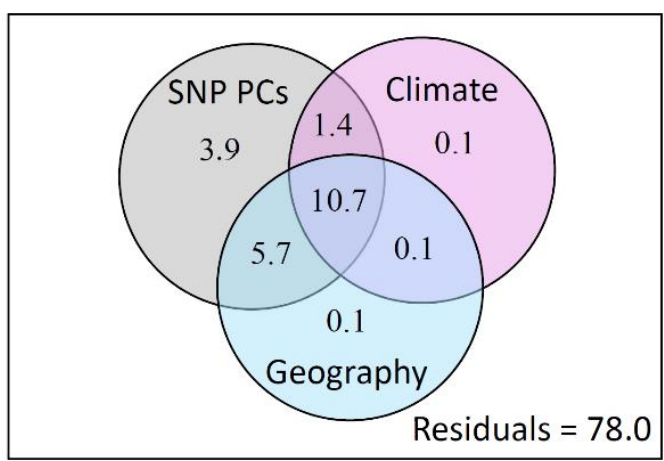

Figure 3.7 Partitioning of variance components in RDA analyses. (a) SNP Clim + Geo model. 7.6\% of the total variation is explained by climate and geography (matrix of spatial variable). (b) Pheno $\sim \mathrm{SNP}+$ Clim + Geo model. $22 \%$ of the total variation is explained by SNP EVs, climate and geography.

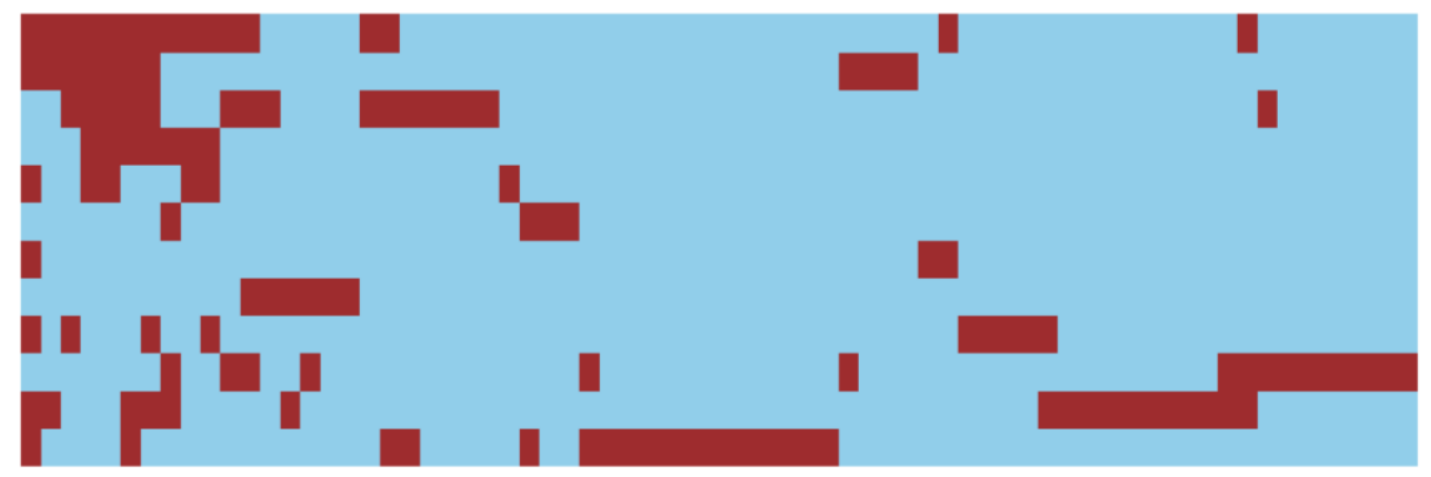
All traits LeafAR PetL Leaf area PetDiam SLA SPAD $\mathrm{Cl}$ Height Stomata Bud set BF

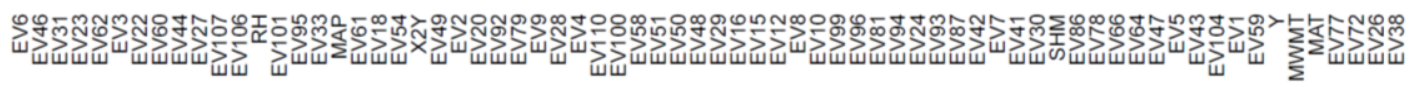

Figure 3.8 Comparison of significant explanatory variables (brown) from RDA model with all 11 traits and the corresponding simple multiple linear regression models. EVs represent SNP eigenvectors, Y represents a space variable, MAT, MWMT, and RH are climate variables. 


\subsection{Supplementary tables and figures}

Supplementary Table S 3.1 Pearson correlation (r) of significant SNP eigenvectors from Clim and

Pheno RDA models (Model 3 and 4) with selected geo-climate and first 4 PCs of 26 geo-climate variables. Values with variable color coding are $r$ values with less than or greater than 0.12 being significant based on Bonferroni correction criteria at 5\% level of significance.

\begin{tabular}{|c|c|c|c|c|c|c|c|c|c|c|c|c|c|}
\hline SNP EV & $\%$ Var* & Longitude & Latitude & Elevation & MAT & MWMT & MAP & SHM & RH & Clim_PC1 & Clim_PC2 & Clim_PC3 & Clim_PC4 \\
\hline EV1 & 1.639 & 0.258 & 0.468 & 0.143 & -0.231 & -0.187 & 0.179 & -0.440 & 0.430 & -0.271 & -0.349 & 0.111 & 0.124 \\
\hline EV2 & & .286 & 0.568 & 0.124 & -0.409 & -0.259 & 0.456 & -0.490 & 0.224 & -0.467 & -0.238 & 0.343 & 0.063 \\
\hline EV3 & & -0.569 & 0.311 & 0.360 & -0.478 & -0.288 & 0.195 & 0.128 & 0.028 & -0.443 & 0.243 & -0.029 & 0.144 \\
\hline EV5 & 407 & .228 & 0.015 & 0.092 & -0.025 & 0.118 & 0.252 & -0.088 & 0.105 & -0.047 & -0.129 & 0.231 & -0.024 \\
\hline EV6 & 284 & 0.276 & -0.229 & -0.140 & 0.262 & 0.166 & 0.146 & -0.052 & 0.032 & 0.226 & -0.245 & 0.051 & -0.240 \\
\hline EV8 & 38 & .167 & 0.089 & -0.230 & 0.071 & -0.061 & -0.201 & 0.012 & -0.033 & .073 & -0.020 & .151 & 0.294 \\
\hline EV18 & 199 & -0.007 & -0.074 & 025 & 0.048 & -0.117 & 0.127 & -0.176 & -0.036 & 0.015 & -0.163 & -0.141 & -0.140 \\
\hline EV22 & & .016 & -0.093 & .065 & 53 & -0.038 & 0.012 & -0.043 & -0.052 & 43 & -0.045 & -0.111 & -0.054 \\
\hline EV23 & 187 & -0.048 & 0.047 & 0.026 & -0.052 & -0.027 & -0.056 & 0.017 & -0.030 & -0.040 & 0.065 & -0.001 & 0.055 \\
\hline EV27 & 181 & 0.004 & 0.006 & -0.010 & -0.024 & -0.014 & 0.056 & 0.018 & -0.024 & -0.025 & 0.017 & 0.036 & -0.037 \\
\hline EV32 & & .024 & -0.051 & -0.067 & 0.077 & 0.130 & -0.149 & 0.213 & -0.156 & 0.100 & 0.180 & 0.036 & 0.039 \\
\hline EV44 & 166 & 0.001 & -0.020 & 0.056 & -0.014 & 0.010 & 0.040 & -0.008 & 0.014 & -0.013 & 0.004 & 0.027 & -0.058 \\
\hline EV46 & 165 & 0.036 & 0.004 & -0.034 & 0.032 & -0.005 & 0.099 & -0.066 & 0.160 & 0.013 & -0.152 & 0.012 & 0.020 \\
\hline EV59 & 156 & -0.014 & -0.016 & -0.011 & 0.012 & -0.021 & 0.000 & 0.016 & 0.043 & 0.012 & -0.021 & -0.048 & 0.035 \\
\hline EV60 & 156 & 0.008 & 0.008 & -0.024 & -0.002 & -0.001 & 0.007 & -0.021 & 0.007 & -0.004 & -0.010 & 0.009 & 0.003 \\
\hline EV62 & & .014 & -0.041 & -0.007 & 0.040 & -0.025 & 0.080 & -0.036 & 0.070 & 0.026 & -0.108 & -0.045 & -0.034 \\
\hline EV72 & .152 & 0.012 & -0.035 & 0.016 & 0.025 & -0.022 & 0.068 & -0.026 & 0.050 & 0.013 & -0.074 & -0.044 & -0.042 \\
\hline EV93 & 144 & -0.004 & 0.000 & -0.036 & 0.027 & 0.043 & -0.029 & 0.034 & -0.009 & .030 & 0.017 & 0.024 & 0.038 \\
\hline EV106 & & -0.024 & 0.007 & & 00 & .002 & -0.015 & 0.006 & -0.008 & 0.000 & 0.011 & 0.003 & 0.019 \\
\hline EV107 & 0.141 & -0.021 & 0.018 & -0.029 & 0.012 & 0.022 & -0.023 & -0.001 & 0.010 & 0.011 & -0.002 & 0.019 & 0.047 \\
\hline
\end{tabular}

*Percentage of total variance explained by SNP eigenvectors 
(a)

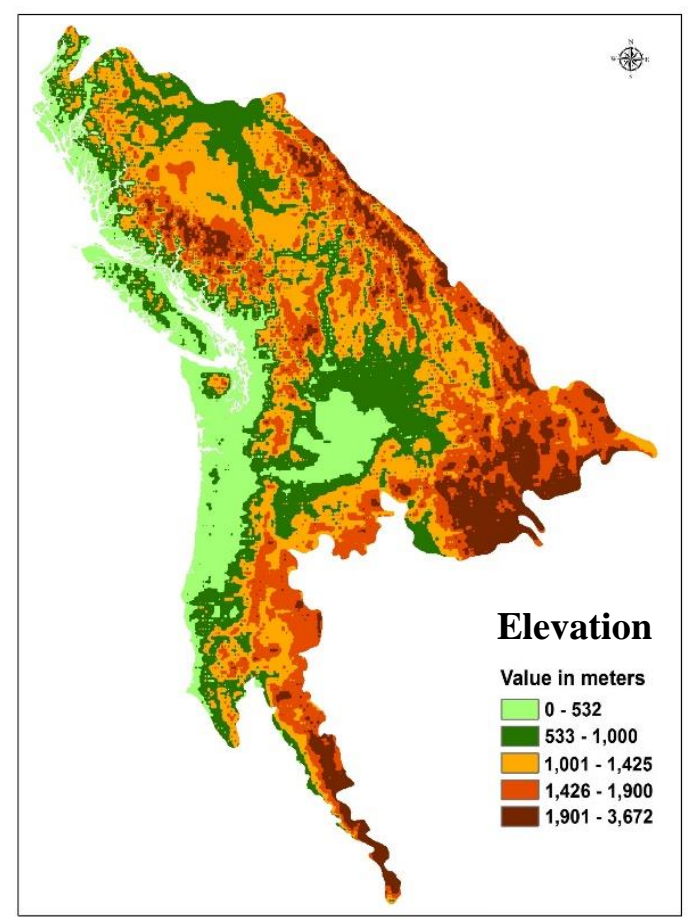

(c)

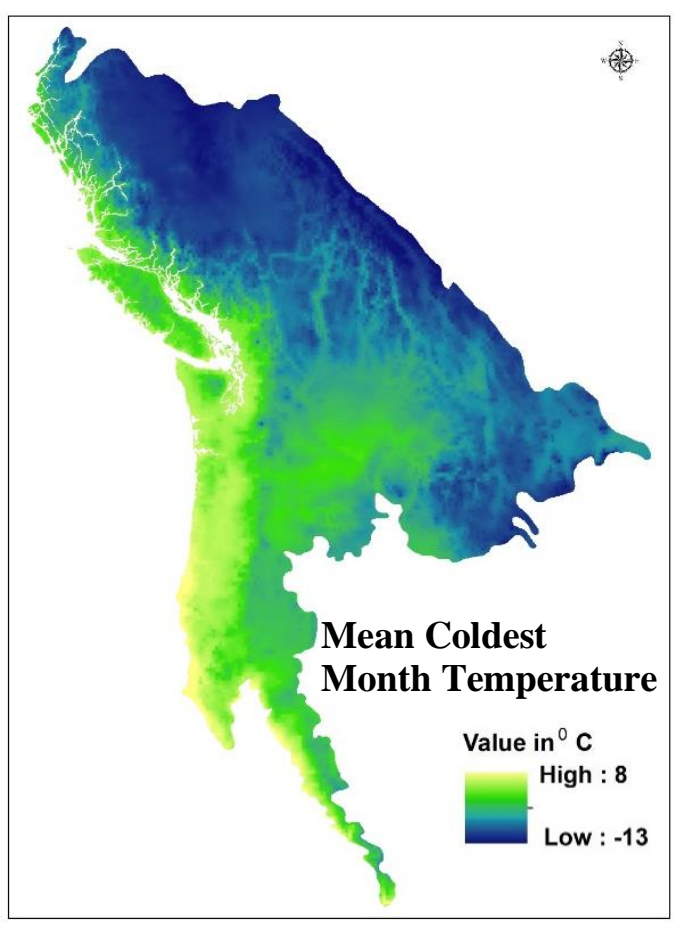

(b)

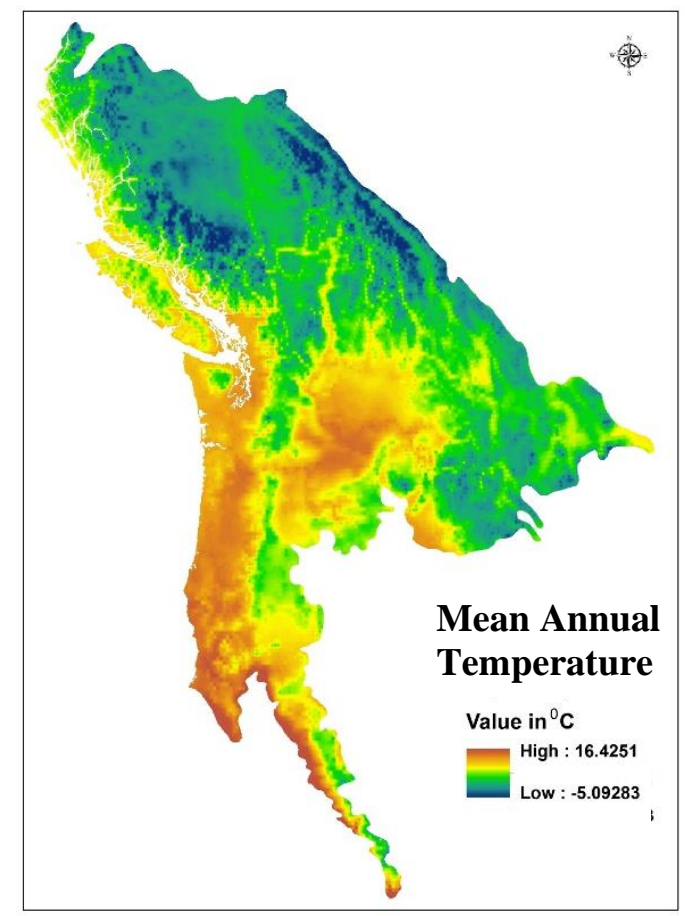

(d)

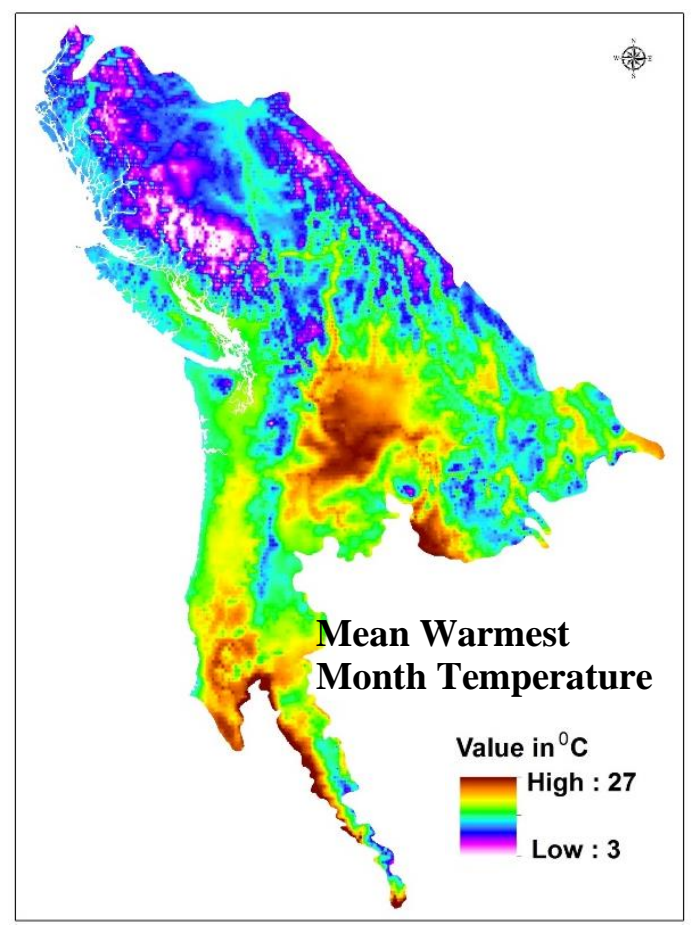


(e)

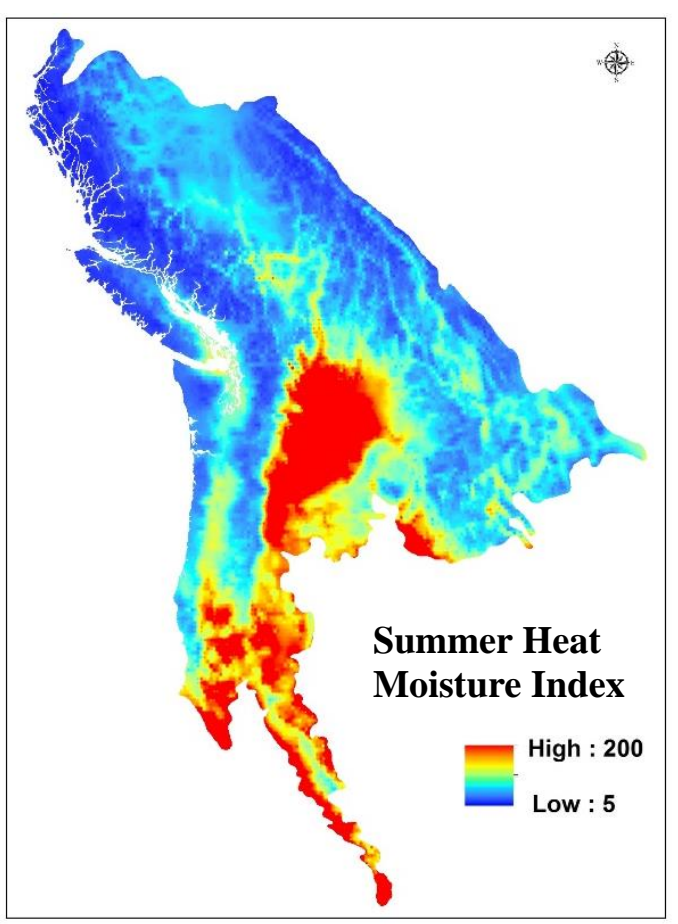

(g)

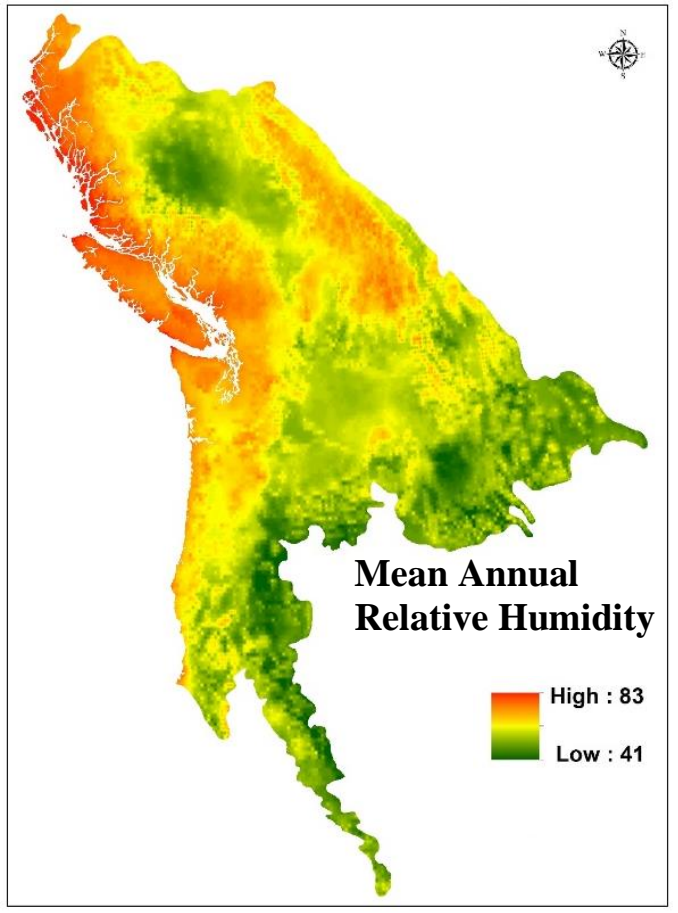

(f)

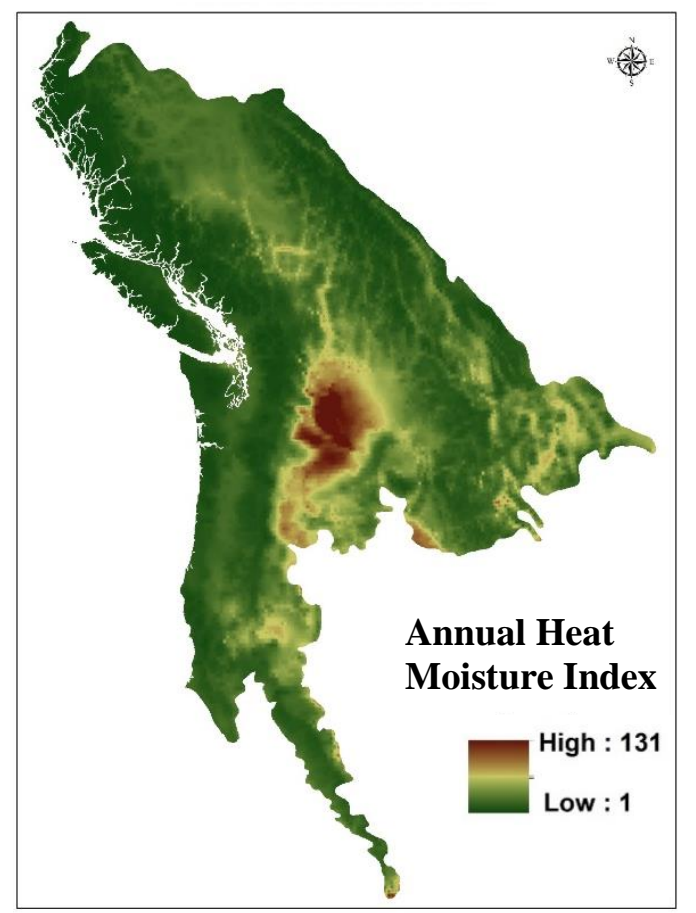

\section{Supplementary Figure S 3.1 Geography}

and climate maps for the range of $P$.

trichocarpa distribution. (a) Elevation; (b)

Mean annual temperature (MAT); (c) Mean

coldest month temperature (MCMT); (d)

Mean warmest month temperature (MWMT);

(e) Summer heat:moisture index (SHM); (f)

Annual heat:moisture index (AHM); (g)

Mean annual relative humidity $(\mathrm{RH})$. 
(a)

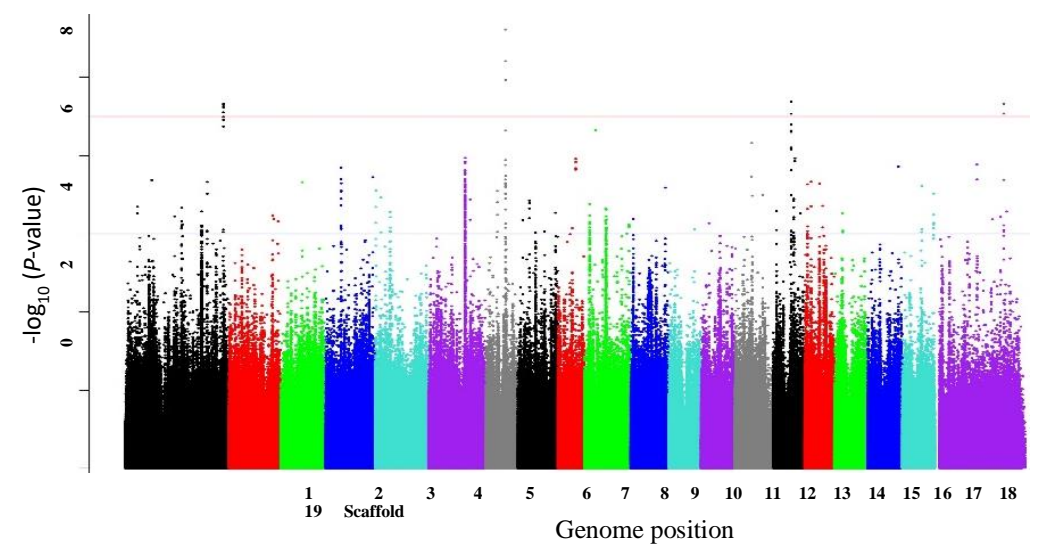

(c)

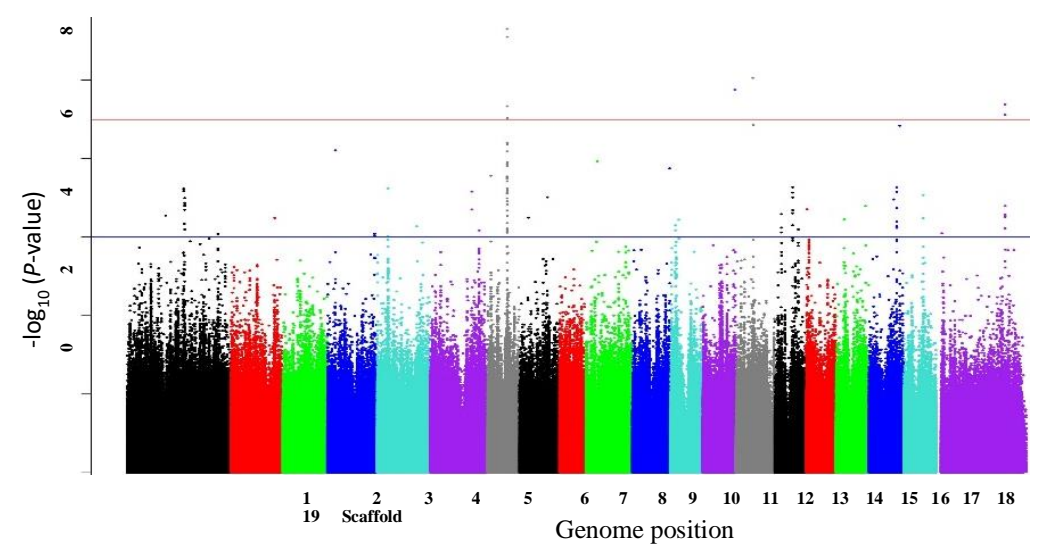

(e)

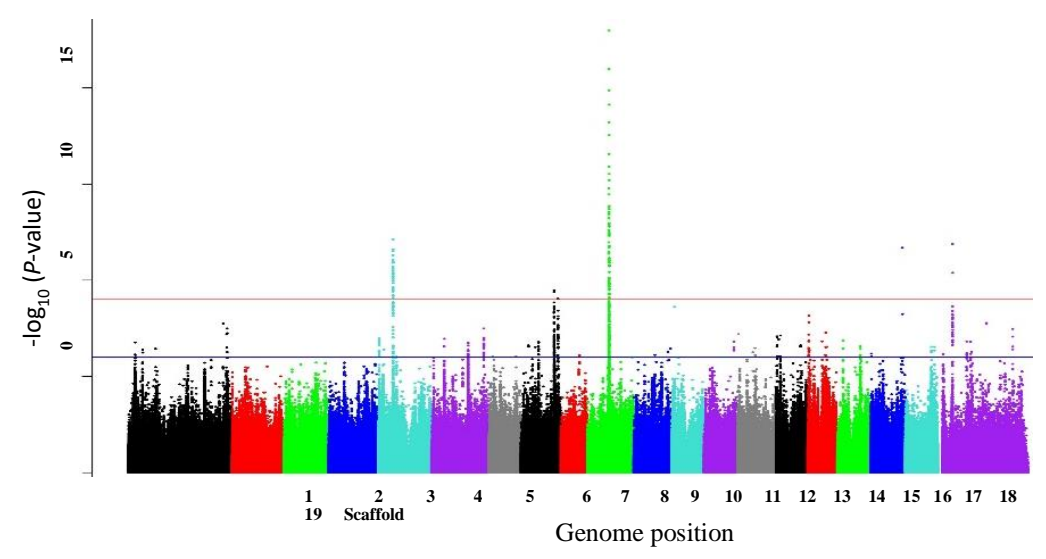

(b)

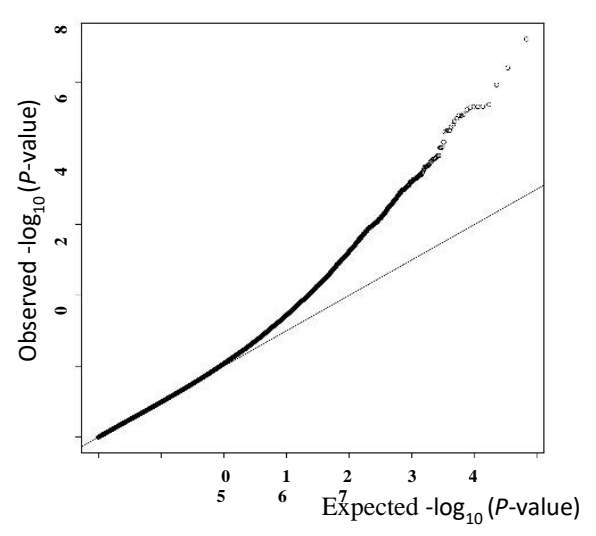

(d)

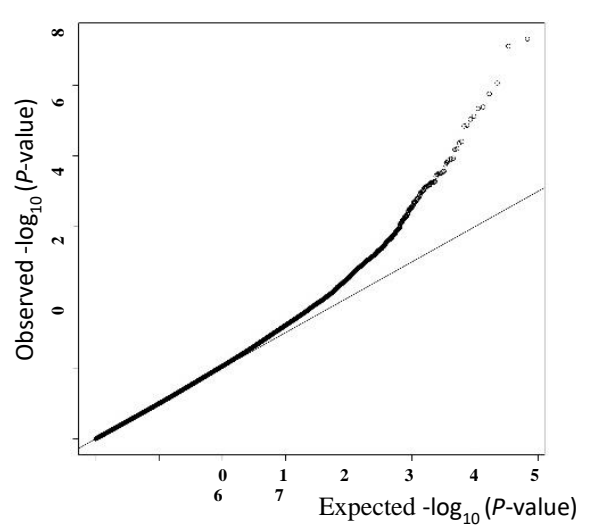

(f)

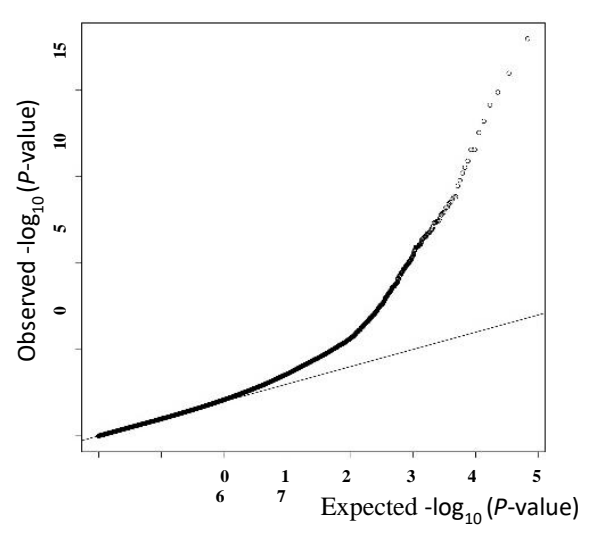


(g)

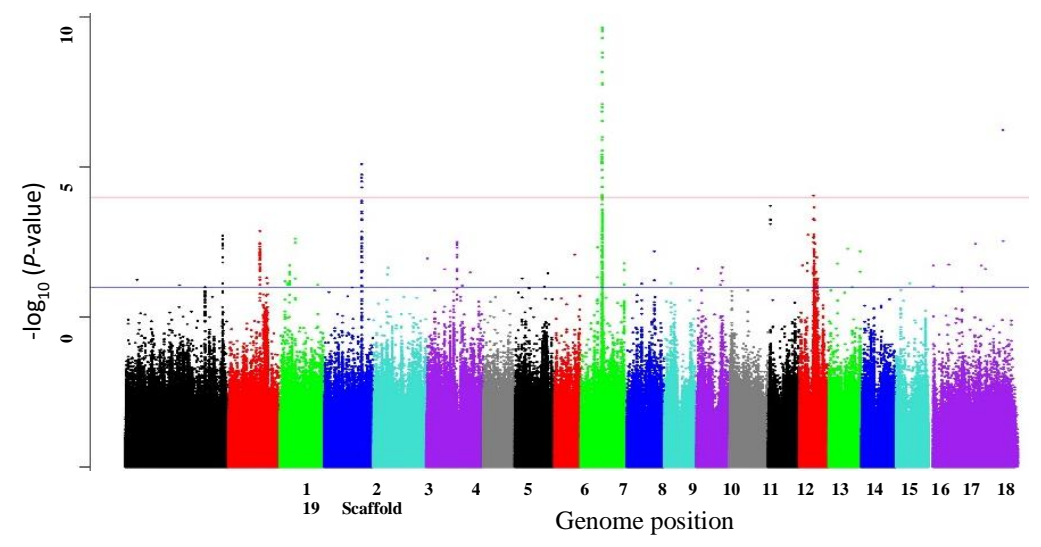

(i)

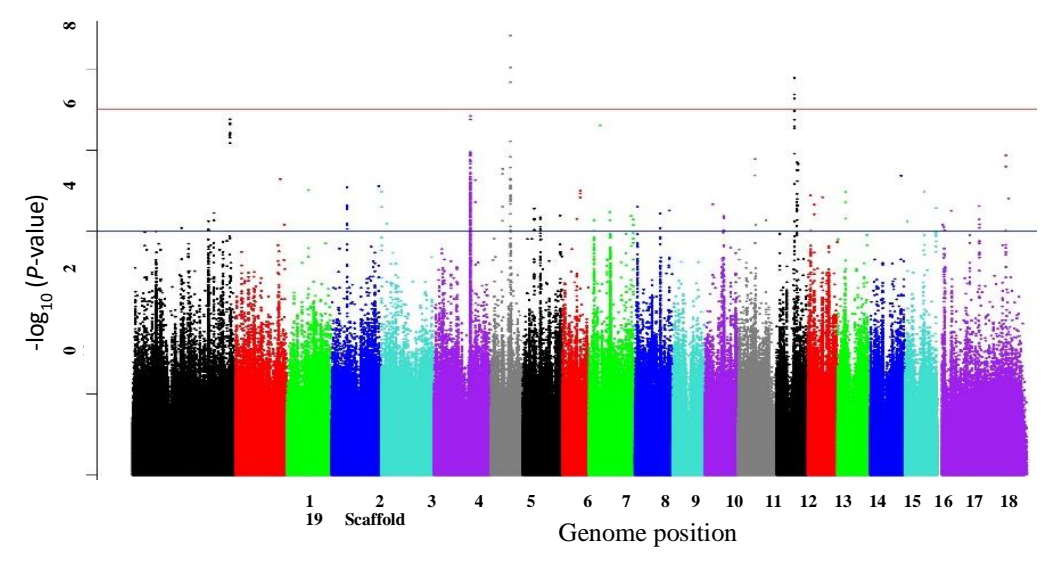

(h)

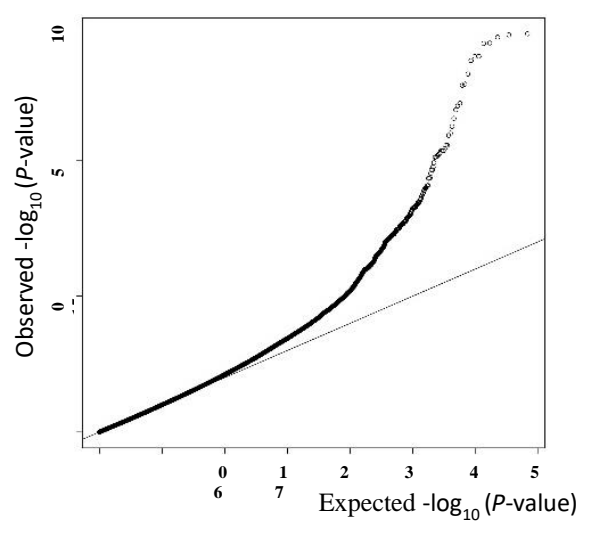

(j)

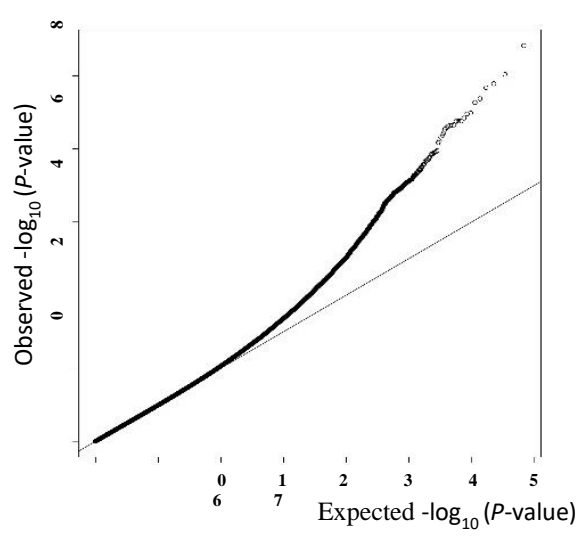

Supplementary Figure S 3.2 Single trait GWAS showing the association of climate variables with the SNPs in the genome - Manhattan (left) and QQ (right) plots. Numbers 1 to 19 represent chromosomes; scaffolds are the reads that did not align to any of the 19 chromosomes. (a) and (b) MAT (Mean annual temperature); (c) and (d) MWMT (Mean warmest month temperature); (e) and (f) TD (Temperature difference between MWMT and MCMT (Mean coldest month temperature); (g) and (h) RH (Mean annual relative humidity); (i) and (j) PC1 of 25 geo-climate variables (includes latitude, longitude and elevation, and 22 climate variables). Red horizontal line indicates Bonferroni correction threshold $\left(P=7.417 \times 10^{-9}\right)$ and blue horizontal line indicates suggestive association threshold $\left(P=1 \times 10^{-7}\right)$. 
(a)

SNP EVs Clim

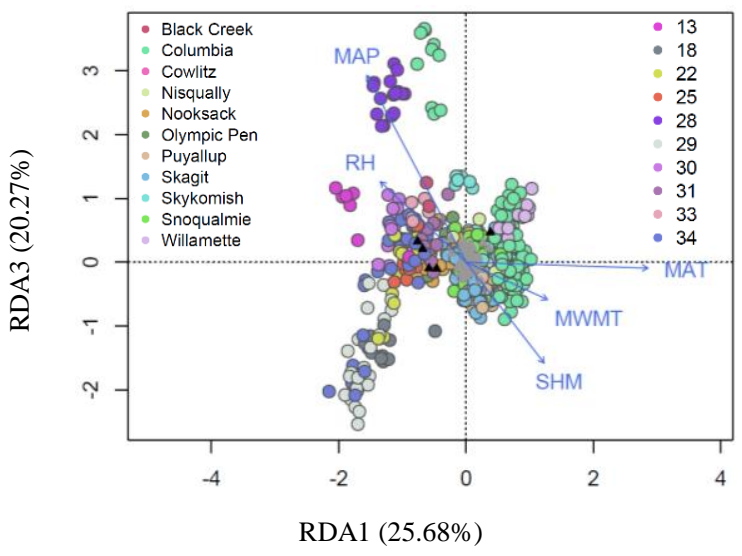

(c)

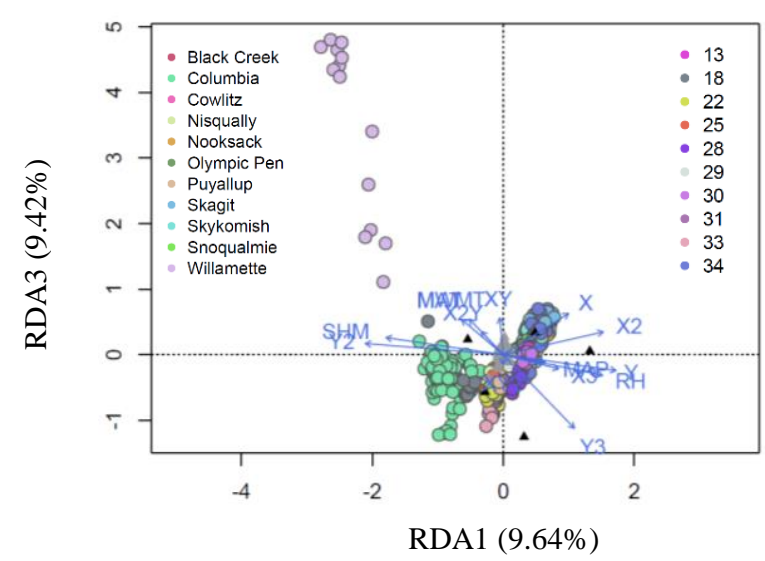

(e)

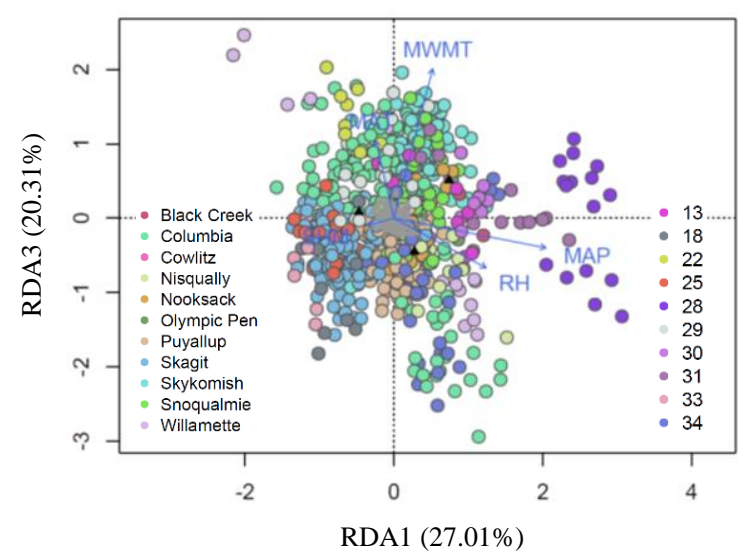

(b)

SNP EVs $\sim$ Clim

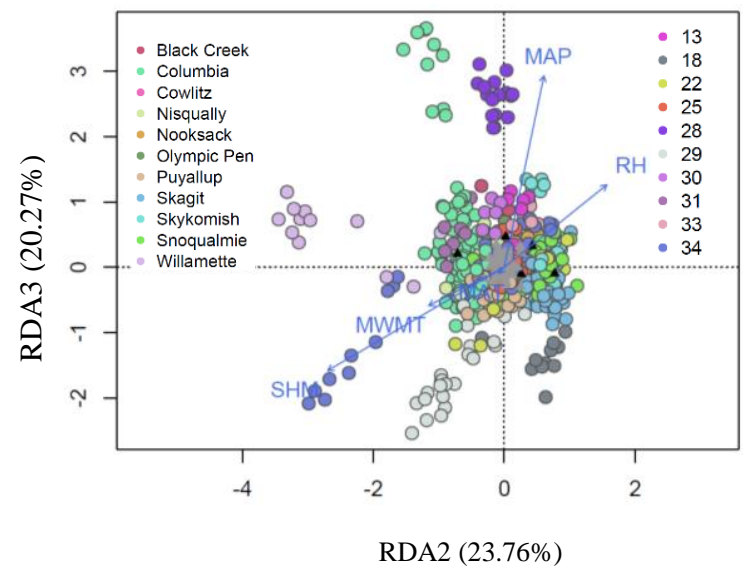

(d)

SNP EVs $\sim$ Clim + Space

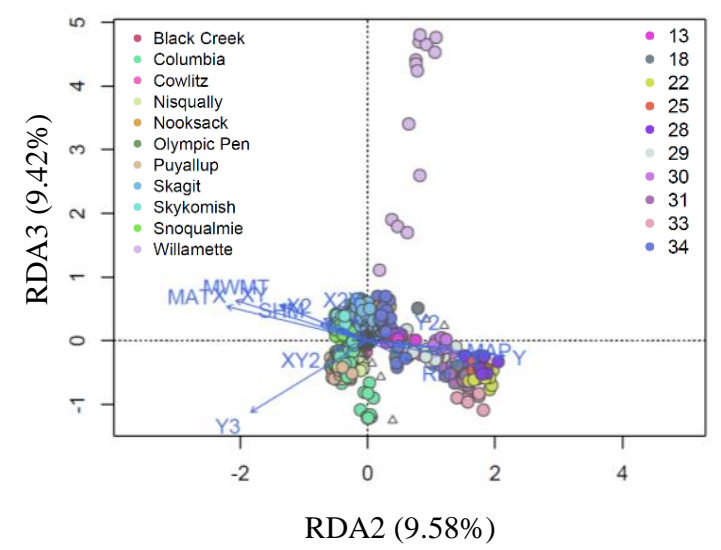

(f) SNP EVs $\sim$ Clim + Condition (Space)

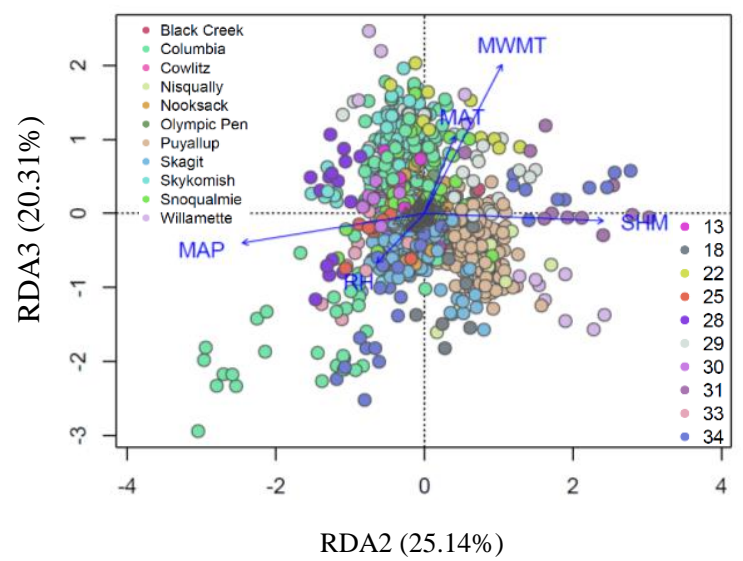


Supplementary Figure S 3.3 RDA triplot with the response matrix of 113 SNP eigenvectors constrained by the matrix of climate variables [(a) and (b)], climate and space variables [(c) and (d)] and climate variables conditioned on space variables [(e) and (f)]. Circles indicate 869 P. trichocarpa genotypes (color coded by population). Triangles indicate SNP eigenvectors with outlier eigenvectors (based on a 3 standard deviation cutoff selected from the tails of the distribution of RDA axes) in black. Blue arrows indicate the influence of climate variables on RDA axis.

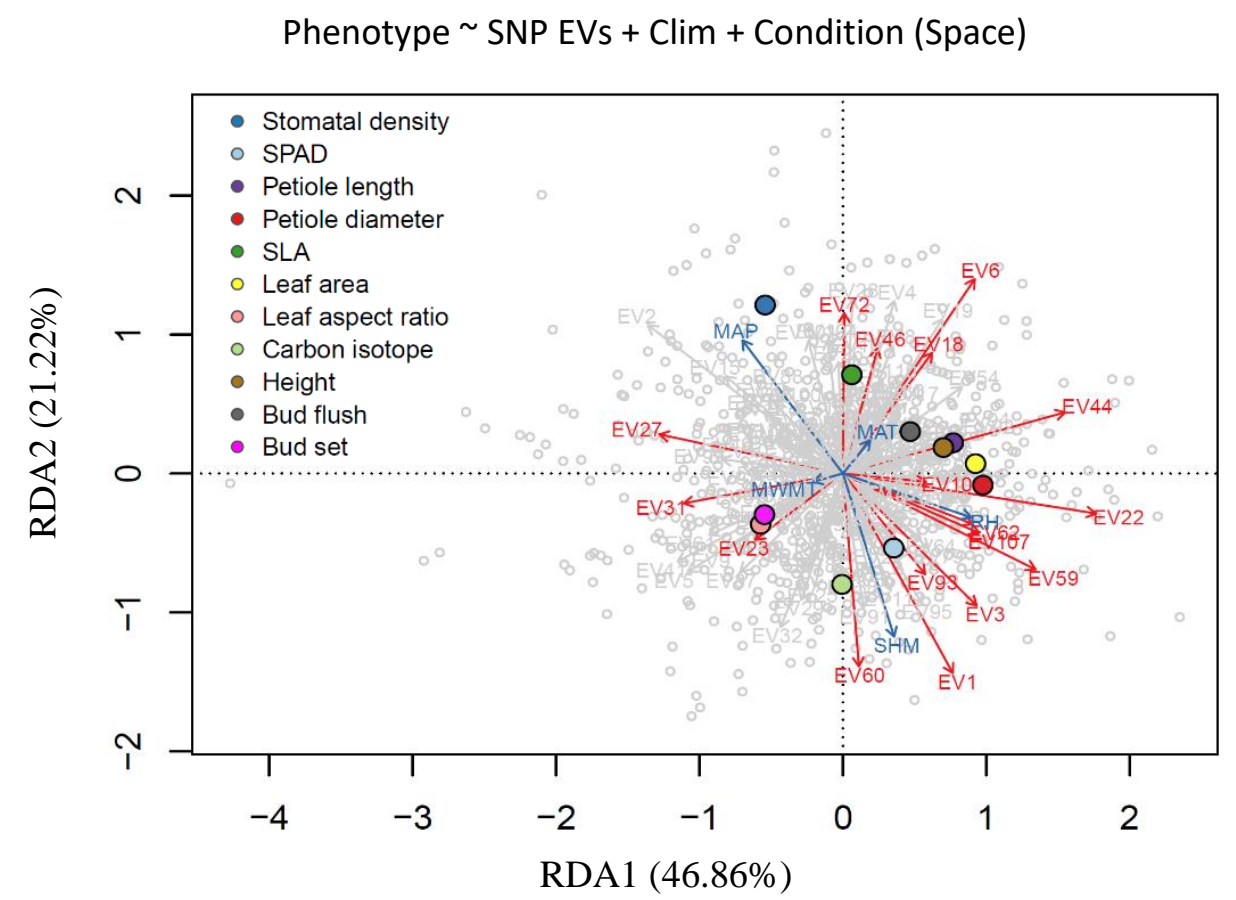

Supplementary Figure S 3.4 RDA triplot with the response matrix of 11 phenotypic traits (large colored circles) constrained by the matrices of 113 SNP eigenvectors and 5 climate variables with the spatial matrix used as covariate. Arrows indicate variable containing SNP eigenvectors and climate with blue indicating climate variables and red indicating significant variables in the RDA model. Small gray circles indicate 869 P. trichocarpa genotypes. 
(a)

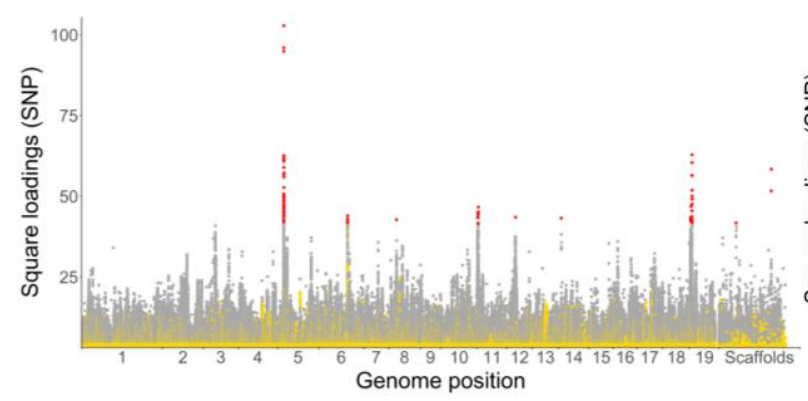

(c)

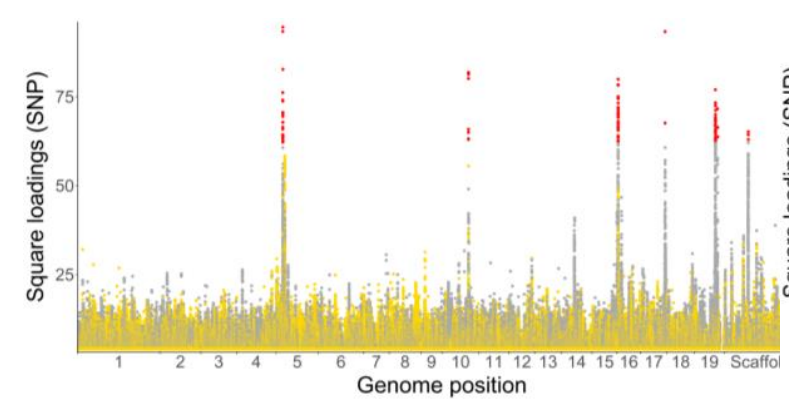

(b)

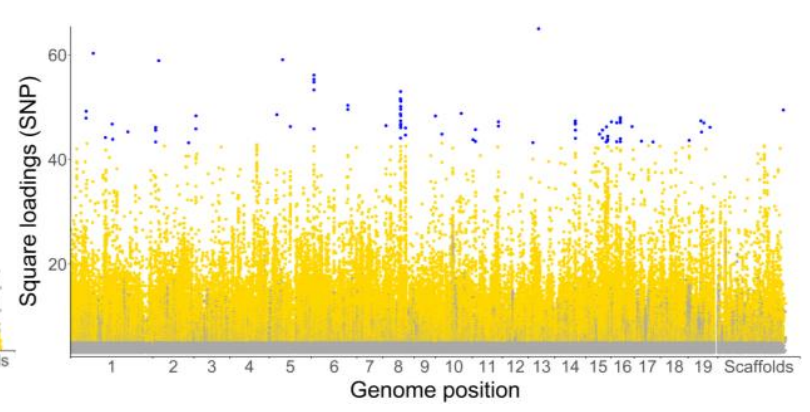

(d)

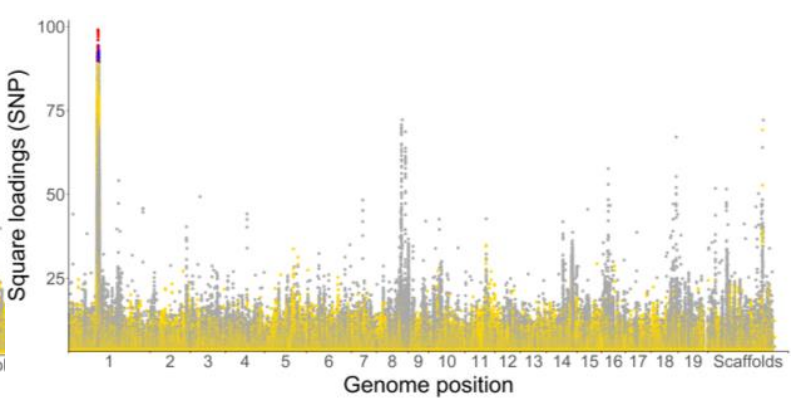

Supplementary Figure S 3.5 Square loadings of top 0.1\% SNPs of outlier eigenvectors (EVs) detected from the climate RDA analysis (a) EV2 (b) EV3 (c) EV5 (d) EV8. Numbers 1 to 19 represent chromosomes; scaffolds are the reads that did not align to any of the 19 chromosomes. Gray and yellow colors represent positive and negative square loadings, respectively. Red and blue indicate top 100 square loadings corresponding to positive and negative loadings, respectively.

(a)

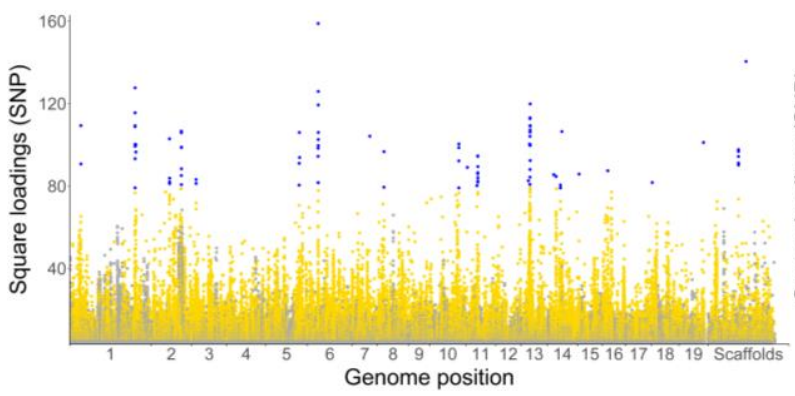

(b)

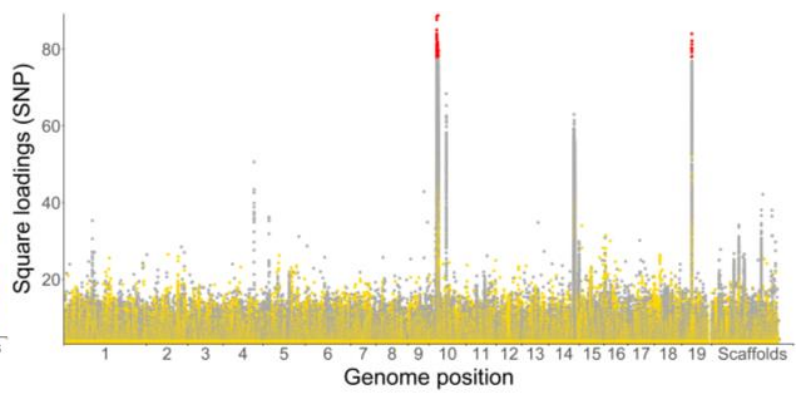


(c)

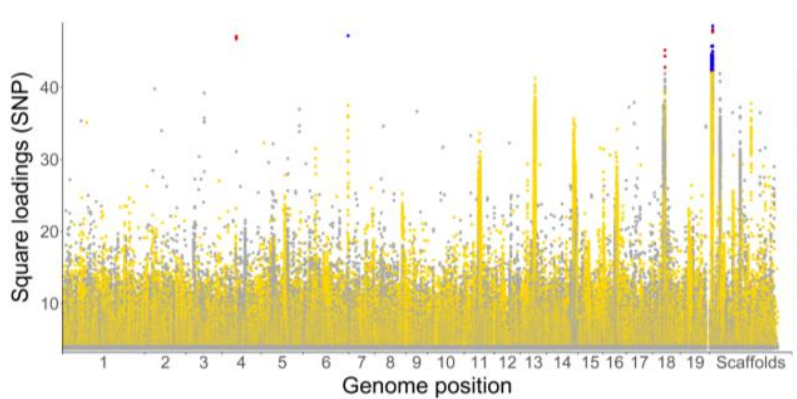

(e)

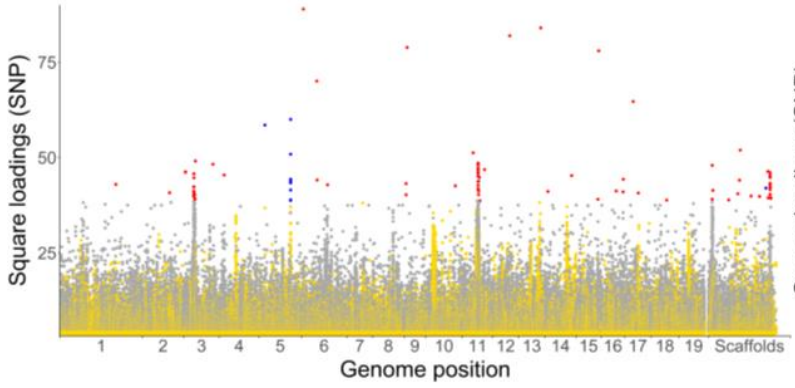

(g)

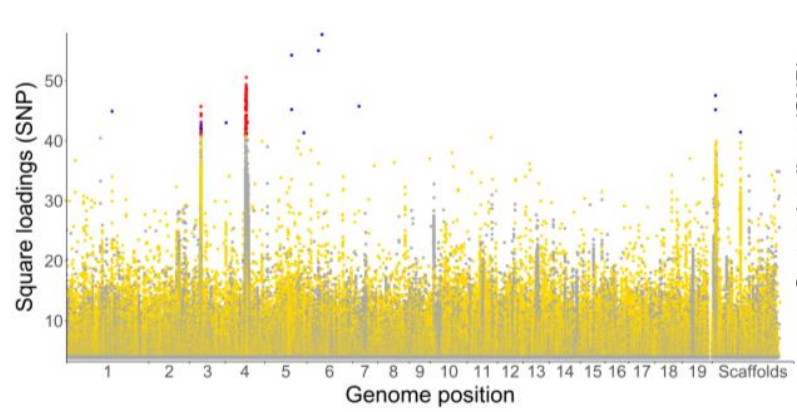

(i)

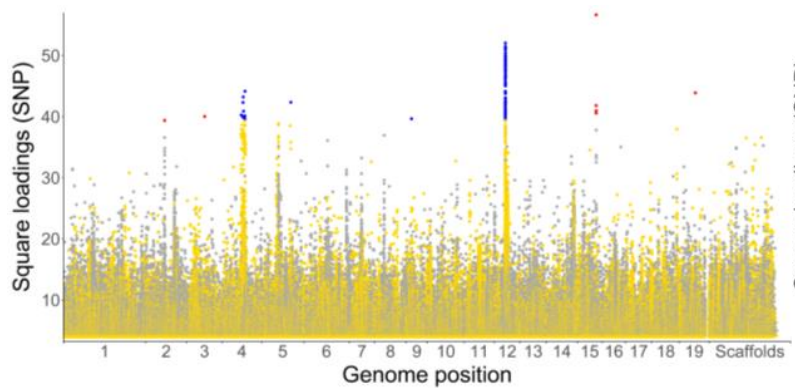

(d)

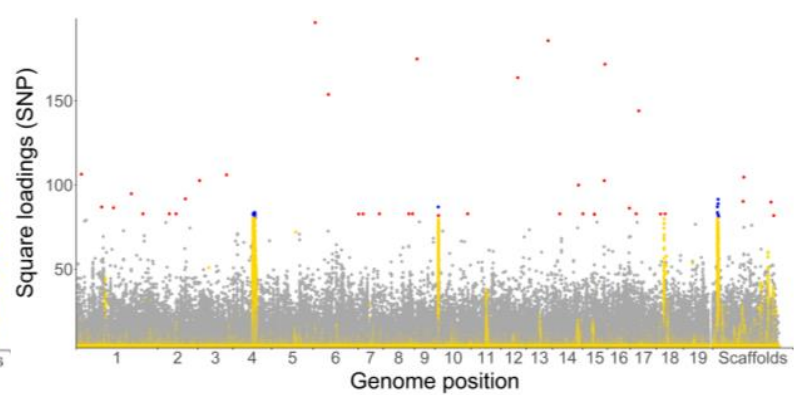

(f)

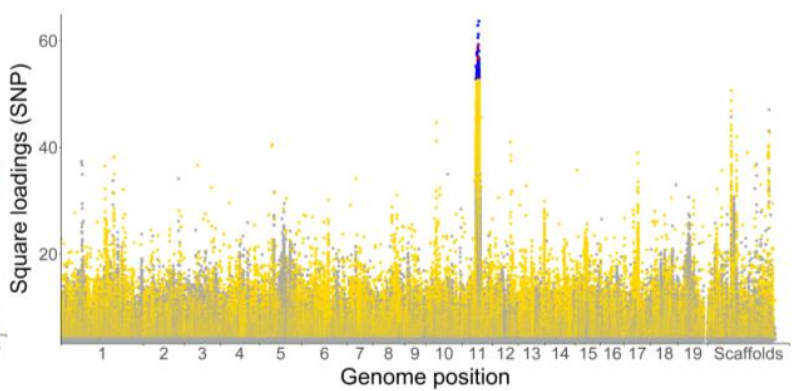

(h)

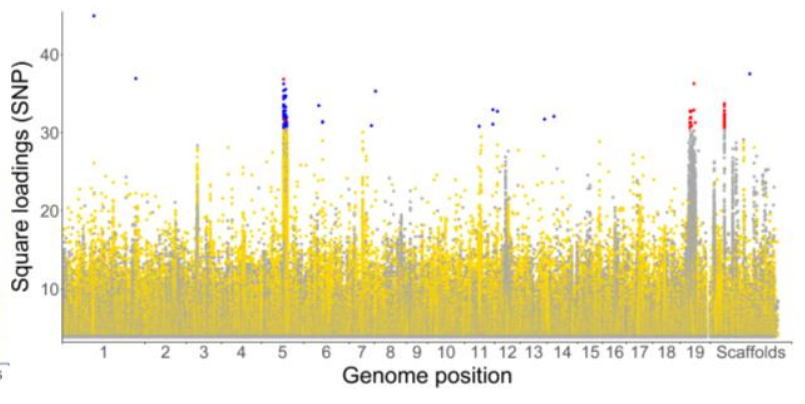

(j)

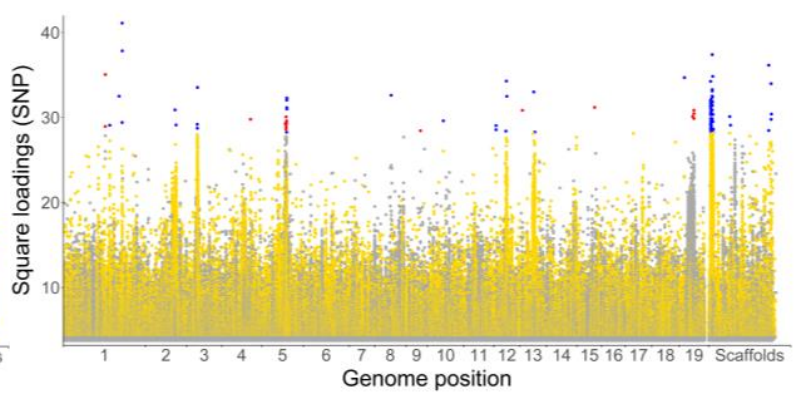


(k)

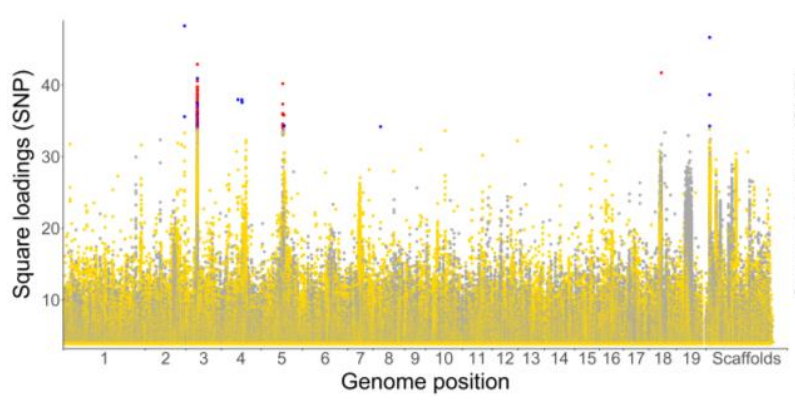

$(\mathrm{m})$

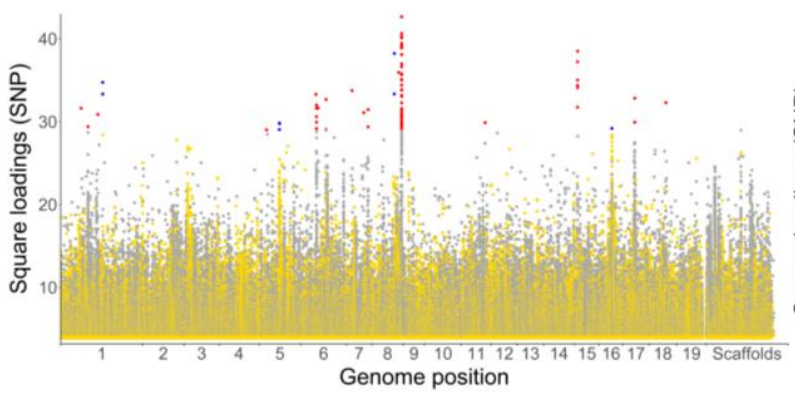

(o)

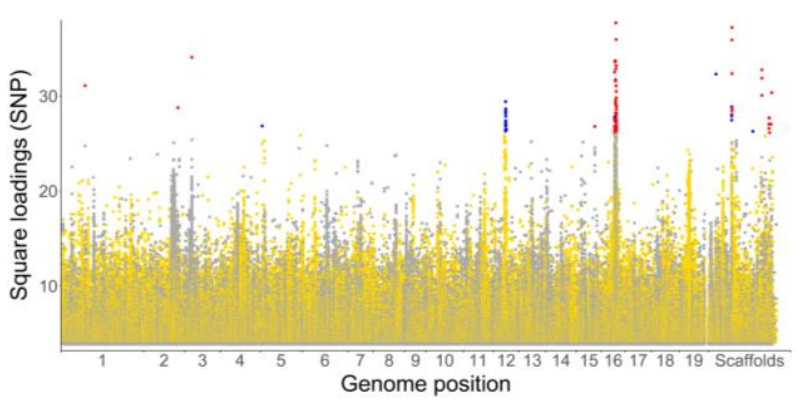

(l)

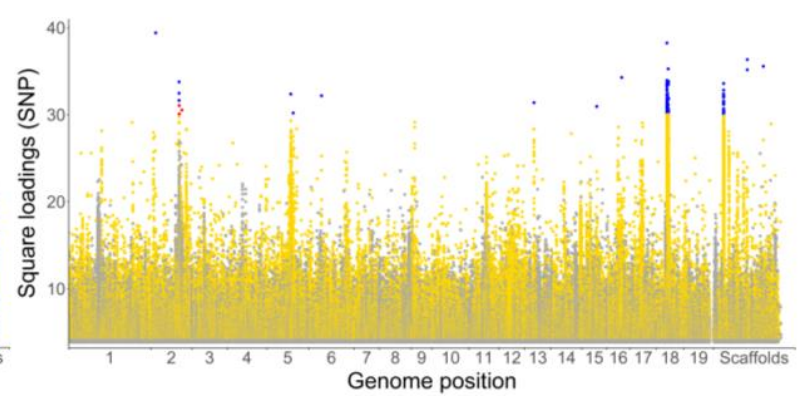

(n)

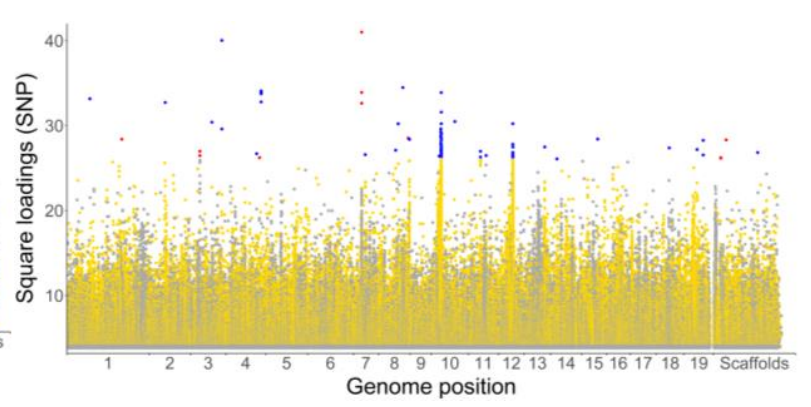

Supplementary Figure S 3.6 Square loadings of top 0.1\% SNPs of outlier eigenvectors (EVs) detected from the phenotypic RDA analysis (a) EV6 (b) EV18 (c) EV22 (d) EV23 (e) EV27 (f) EV31 (g) EV44 (h) EV46 (i) EV59 (j) EV60 (k) EV62 (1) EV72 (m) EV93 (n) EV106 (0) EV107. Numbers 1 to 19 represent chromosomes; scaffolds are the reads that did not align to any of the 19 chromosomes. Gray and yellow colors represent positive and negative square loadings, respectively. Red and blue indicate top 100 square loadings corresponding to positive and negative loadings, respectively. 


\subsection{References}

Aitken, S. N., Yeaman, S., Holliday, J. A., Wang, T., \& Curtis-McLane, S. (2008). Adaptation, migration or extirpation: climate change outcomes for tree populations. Evolutionary Applications, 1(1), 95111. doi: 10.1111/j.1752-4571.2007.00013.x

Berg, J. J., \& Coop, G. (2014). A Population Genetic Signal of Polygenic Adaptation. PLoS Genetics, 10(8). doi: 10.1371/journal.pgen.1004412

Boyle, E. A., Li, Y. I., \& Pritchard, J. K. (2017). An Expanded View of Complex Traits: From Polygenic to Omnigenic. Cell, 169(7), 1177-1186. doi: 10.1016/j.cell.2017.05.038

Bragg, J. G., Supple, M. A., Andrew, R. L., \& Borevitz, J. O. (2015). Genomic variation across landscapes: insights and applications. New Phytologist, 207, 953-967. doi: 10.1111/nph.13410

Brauer, C. J., Hammer, M. P., \& Beheregaray, L. B. (2016). Riverscape genomics of a threatened fish across a hydroclimatically heterogeneous river basin. Molecular Ecology, 25(20), 5093-5113. doi: $10.1111 / \mathrm{mec} .13830$

Ćalić, I., Bussotti, F., Martínez-García, P. J., \& Neale, D. B. (2016). Recent landscape genomics studies in forest trees—what can we believe? Tree Genetics and Genomes, 12(1), 1-7. doi: 10.1007/s11295015-0960-0

Capblancq, T., Luu, K., Blum, M. G. B., \& Bazin, E. (2018). Evaluation of redundancy analysis to identify signatures of local adaptation. Molecular Ecology Resources, 18(6), 1223-1233. doi: 10.1111/1755-0998.12906

Cavalli-Sforza, L. . L. . (1996). Population Structure and Human Evolution. Proceedings of the Royal Society of London. Series B, Biological Sciences, 164(995), 362-379. Royal Society.

Chhetri, H. B., Macaya-Sanz, D., Kainer, D., Biswal, A. K., Evans, L. M., Chen, J. G., ... DiFazio, S. P. (2019). Multitrait genome-wide association analysis of Populus trichocarpa identifies key polymorphisms controlling morphological and physiological traits. New Phytologist, 223, 293-309. doi: 10.1111/nph.15777

Dahl, A., Iotchkova, V., Baud, A., Johansson, S., Gyllensten, U., Soranzo, N., .. Marchini, J. (2016). A multiple-phenotype imputation method for genetic studies. Nature Genetics, 48(4), 466-472. doi: 10.1038/ng.3513

De Kort, H., Vandepitte, K., Bruun, H. H., Closset-Kopp, D., Honnay, O., \& Mergeay, J. (2014). Landscape genomics and a common garden trial reveal adaptive differentiation to temperature across Europe in the tree species Alnus glutinosa. Molecular Ecology, 23(19), 4709-4721. doi: $10.1111 /$ mec. 12813

De Mita, S., Thuillet, A. C., Gay, L., Ahmadi, N., Manel, S., Ronfort, J., \& Vigouroux, Y. (2013). Detecting selection along environmental gradients: Analysis of eight methods and their effectiveness 
for outbreeding and selfing populations. Molecular Ecology, 22(5), 1383-1399. doi:

$10.1111 / \mathrm{mec} .12182$

De Villemereuil, P., Gaggiotti, O. E., Mouterde, M., \& Till-Bottraud, I. (2016). Common garden experiments in the genomic era: New perspectives and opportunities. Heredity, 116(3), 249-254. doi: $10.1038 / \mathrm{hdy} .2015 .93$

Eckert, A. J., Maloney, P. E., Vogler, D. R., Jensen, C. E., Mix, A. D., \& Neale, D. B. (2015). Local adaptation at fine spatial scales: an example from sugar pine (Pinus lambertiana, Pinaceae). Tree Genetics and Genomes, 11(3). doi: 10.1007/s11295-015-0863-0

Eckert, A. J., Van Heerwaarden, J., Wegrzyn, J. L., Nelson, C. D., Ross-Ibarra, J., González-Martínez, S. C., \& Neale, D. B. (2010). Patterns of population structure and environmental associations to aridity across the range of loblolly pine (Pinus taeda L., Pinaceae). Genetics, 185(3), 969-982. doi: 10.1534/genetics.110.115543

Eckert, A. J., Wegrzyn, J. L., Pande, B., Jermstad, K. D., Lee, J. M., Liechty, J. D., ... Neale, D. B. (2009). Multilocus patterns of nucleotide diversity and divergence reveal positive selection at candidate genes related to cold hardiness in coastal Douglas fir (Pseudotsuga menziesii var. menziesii). Genetics, 183(1), 289-298. doi: 10.1534/genetics.109.103895

Evans, L. M., Slavov, G. T., Rodgers-Melnick, E., Martin, J., Ranjan, P., Muchero, W., ... DiFazio, S. P. (2014). Population genomics of Populus trichocarpa identifies signatures of selection and adaptive trait associations. Nature Genetics, 46(10), 1089-1096. doi: 10.1038/ng.3075

Flanagan, S. P., Forester, B. R., Latch, E. K., Aitken, S. N., \& Hoban, S. (2018). Guidelines for planning genomic assessment and monitoring of locally adaptive variation to inform species conservation. Evolutionary Applications, 11(7), 1035-1052. doi: 10.1111/eva.12569

Forester, B. R., Jones, M. R., Joost, S., Landguth, E. L., \& Lasky, J. R. (2016). Detecting spatial genetic signatures of local adaptation in heterogeneous landscapes. Molecular Ecology, 25(1), 104-120. doi: 10.1111/mec.13476

Forester, B. R., Lasky, J. R., Wagner, H. H., \& Urban, D. L. (2018). Comparing methods for detecting multilocus adaptation with multivariate genotype-environment associations. Molecular Ecology, 27(9), 2215-2233. doi: 10.1111/mec.14584

Frichot, E., Schoville, S. D., Bouchard, G., \& François, O. (2013). Testing for associations between loci and environmental gradients using latent factor mixed models. Molecular Biology and Evolution, 30(7), 1687-1699. doi: 10.1093/molbev/mst063

Geraldes, A., Farzaneh, N., Grassa, C. J., Mckown, A. D., Guy, R. D., Mansfield, S. D., .. Cronk, Q. C. B. (2014). Landscape genomics of Populus trichocarpa: The role of hybridization, limited gene flow, and natural selection in shaping patterns of population structure. Evolution, 68(11), 3260- 
3280. doi: 10.1111/evo.12497

Gonzales-Vigil, E., Hefer, C. A., von Loessl, M. E., La Mantia, J., \& Mansfield, S. D. (2017). Exploiting Natural Variation to Uncover an Alkene Biosynthetic Enzyme in Poplar. The Plant Cell, 29(8), 2000-2015. doi: 10.1105/tpc.17.00338

Gugger, P. F., Liang, C. T., Sork, V. L., Hodgskiss, P., \& Wright, J. W. (2018). Applying landscape genomic tools to forest management and restoration of Hawaiian koa (Acacia koa) in a changing environment. Evolutionary Applications, 11(2), 231-242. doi: 10.1111/eva.12534

Guo, N., Cheng, F., Wu, J., Liu, B., Zheng, S., Liang, J., \& Wang, X. (2014). Anthocyanin biosynthetic genes in Brassica rapa. BMC Genomics, 15, 426.

Hancock, A. M., Witonsky, D. B., Alkorta-Aranburu, G., Beall, C. M., Gebremedhin, A., Sukernik, R., ... Di Rienzo, A. (2011). Adaptations to climate-mediated selective pressures in humans. PLoS Genetics, 7(4). doi: 10.1371/journal.pgen.1001375

Hofmeister, N. R., Werner, S. J., \& Lovette, I. (2019). Environment but not geography explains genetic variation in the invasive and largely panmictic European starling in North America. BioRxiv, 1-19. doi: http://dx.doi.org/10.1101/643858

Holliday, J. A., Wang, T., \& Aitken, S. (2013). Predicting Adaptive Phenotypes From Multilocus Genotypes in Sitka Spruce (Picea sitchensis) Using Random Forest. G3 Genes Genomes Genetics, 2(9), 1085-1093. doi: 10.1534/g3.112.002733

Holliday, J. A., Zhou, L., Bawa, R., Zhang, M., \& Oubida, R. W. (2016). Evidence for extensive parallelism but divergent genomic architecture of adaptation along altitudinal and latitudinal gradients in Populus trichocarpa. New Phytologist, 209(3), 1240-1251. doi: 10.1111/nph.13643

Jensen, J. D., Kim, Y., DuMont, V. B., Aquadro, C. F., \& Bustamante, C. D. (2005). Distinguishing between selective sweeps and demography using DNA polymorphism data. Genetics, 170(3), 14011410. doi: 10.1534/genetics.104.038224

Jombart, T., Pontier, D., \& Dufour, A. B. (2009). Genetic markers in the playground of multivariate analysis. Heredity, 102(4), 330-341. doi: 10.1038/hdy.2008.130

Joost, S., Bonin, A., Bruford, M. W., Després, L., Conord, C., Erhardt, G., \& Taberlet, P. (2007). A spatial analysis method (SAM) to detect candidate loci for selection: Towards a landscape genomics approach to adaptation. Molecular Ecology, 16(18), 3955-3969. doi: 10.1111/j.1365294X.2007.03442.x

Lasky, J. R., Des Marais, D. L., McKay, J. K., Richards, J. H., Juenger, T. E., \& Keitt, T. H. (2012). Characterizing genomic variation of Arabidopsis thaliana: The roles of geography and climate. Molecular Ecology, 21(22), 5512-5529. doi: 10.1111/j.1365-294X.2012.05709.x

Le Corre, V., \& Kremer, A. (2012). The genetic differentiation at quantitative trait loci under local 
adaptation. Molecular Ecology, 21(7), 1548-1566. doi: 10.1111/j.1365-294X.2012.05479.x

Legendre, P., \& Legendre, L. (2012). Numerical Ecology. Elsevier.

Lind, B. M., Friedline, C. J., Wegrzyn, J. L., Maloney, P. E., Vogler, D. R., Neale, D. B., \& Eckert, A. J. (2017). Water availability drives signatures of local adaptation in whitebark pine (Pinus albicaulis Engelm.) across fine spatial scales of the Lake Tahoe Basin, USA. Molecular Ecology, 26(12), 3168-3185. doi: 10.1111/mec.14106

Lind, B. M., Menon, M., Bolte, C. E., Faske, T. M., \& Eckert, A. J. (2018). The genomics of local adaptation in trees: are we out of the woods yet? Tree Genetics and Genomes, 14(2). doi: $10.1007 / \mathrm{s} 11295-017-1224-\mathrm{y}$

Liu, Y., Tikunov, Y., Schouten, R. E., Marcelis, L. F. M., Visser, R. G. F., \& Bovy, A. (2018). Anthocyanin Biosynthesis and Degradation Mechanisms in Solanaceous Vegetables: A Review. Frontiers in Chemistry, 6(52). doi: 10.3389/fchem.2018.00052

Lotterhos, K. E., \& Whitlock, M. C. (2015). The relative power of genome scans to detect local adaptation depends on sampling design and statistical method. Molecular Ecology, 24(5), 10311046. doi: $10.1111 / \mathrm{mec} .13100$

Luu, K., Bazin, E., \& Blum, M. G. B. (2017). pcadapt : an R package to perform genome scans for selection based on principal component analysis. 33, 67-77. doi: 10.1111/1755-0998.12592

Mckown, A. D., Guy, R. D., Klápště, J., Geraldes, A., Friedmann, M., Cronk, Q. C. B., ... Douglas, C. J. (2014). Geographical and environmental gradients shape phenotypic trait variation and genetic structure in Populus trichocarpa. New Phytologist, 201(4), 1263-1276. doi: 10.1111/nph.12601

Meirmans, P. G. (2012). The trouble with isolation by distance. Molecular Ecology, 21(12), 2839-2846. doi: 10.1111/j.1365-294X.2012.05578.x

Meirmans, P. G. (2015). Seven common mistakes in populuation and how to avoid them. Molecular Ecology, 2015(24), 3223-3231.

Meirmans, P. G., Godbout, J., Lamothe, M., Thompson, S. L., \& Isabel, N. (2017). History rather than hybridization determines population structure and adaptation in Populus balsamifera. Journal of Evolutionary Biology, 30(11), 2044-2058. doi: 10.1111/jeb.13174

Misyura, M., Colasanti, J., \& Rothstein, S. J. (2013). Physiological and genetic analysis of Arabidopsis thaliana anthocyanin biosynthesis mutants under chronic adverse environmental conditions. Journal of Experimental Botany, 64(1), 229-240. doi: 10.1093/jxb/ers328

Neale, D. B., \& Ingvarsson, P. K. (2008). Population, quantitative and comparative genomics of adaptation in forest trees. Current Opinion in Plant Biology, 11(2), 149-155. doi: 10.1016/j.pbi.2007.12.004

Neale, D. B., \& Kremer, A. (2011). Forest tree genomics: Growing resources and applications. Nature 
Reviews Genetics, 12(2), 111-122. doi: 10.1038/nrg2931

Oksanen, J., Blanchet, F. G., Friendly, M., Kindt, R., Legendre, P., McGlinn, D., ... Wagner, H. (2018). vegan: Community Ecology Package. R package version 2.5-2. Retrieved from https://cran.rproject.org/package=vegan

Oubida, R. W., Gantulga, D., Zhang, M., Zhou, L., Bawa, R., \& Holliday, J. A. (2015). Partitioning of multivariate phenotypes using regression trees reveals complex patterns of adaptation to climate across the range of black cottonwood (Populus trichocarpa). Frontiers in Plant Science, 6(March), 181. doi: $10.3389 /$ fpls.2015.00181

Patterson, N., Price, A. L., \& Reich, D. (2006). Population structure and eigenanalysis. PLoS Genetics, 2(12), 2074-2093. doi: 10.1371/journal.pgen.0020190

Prunier, J., Verta, J. P., \& Mackay, J. J. (2016). Conifer genomics and adaptation: At the crossroads of genetic diversity and genome function. New Phytologist, 209(1), 44-62. doi: 10.1111/nph.13565

R Core Team. (2017). R: A language and environment for statistical computing. R Foundation for Statistical Computing, Vienna, Austria. Retrieved from http://www.r-project.org/

Rajora, O. P., Eckert, A. J., \& Zinck, J. W. R. (2016). Single-locus versus multilocus patterns of local adaptation to climate in eastern white pine (Pinus strobus, Pinaceae). PLoS ONE, 11(7), 1-26. doi: 10.1371/journal.pone.0158691

Rellstab, C., Gugerli, F., Eckert, A. J., Hancock, A. M., \& Holderegger, R. (2015). A practical guide to environmental association analysis in landscape genomics. Molecular Ecology, 24(17), 4348-4370. doi: $10.1111 / \mathrm{mec} .13322$

Richardson, J. L., Urban, M. C., Bolnick, D. I., \& Skelly, D. K. (2014). Microgeographic adaptation and the spatial scale of evolution. Trends in Ecology and Evolution, 29(3), 165-176. doi: 10.1016/j.tree.2014.01.002

Savolainen, O., Lascoux, M., \& Merilä, J. (2013). Ecological genomics of local adaptation. Nature Reviews. Genetics, 14(11), 807-820. doi: 10.1038/nrg3522

Savolainen, O., \& Pyhäjärvi, T. (2007). Genomic diversity in forest trees. Current Opinion in Plant Biology, 10(2), 162-167. doi: 10.1016/j.pbi.2007.01.011

Schindelin, J., Rueden, C. T., Hiner, M. C., \& Eliceiri, K. W. (2015). The ImageJ ecosystem: An open platform for biomedical image analysis. Molecular Reproduction and Development, 82(7-8), 518529. doi: 10.1002/mrd.22489

Shi, M.-Z., \& Xie, D.-Y. (2014). Biosynthesis and Metabolic Engineering of Anthocyanins in Arabidopsis thaliana. Recent Patents on Biotechnology, 8, 47-60.

Slavov, G. T., Difazio, S. P., Martin, J., Schackwitz, W., Muchero, W., Rodgers-Melnick, E., ... Tuskan, G. A. (2012). Genome resequencing reveals multiscale geographic structure and extensive linkage 
disequilibrium in the forest tree Populus trichocarpa. New Phytologist, 196(3), 713-725. doi: 10.1111/j.1469-8137.2012.04258.x

Slavov, G. T., Leonardi, S., Burczyk, J., Adams, W. T., Strauss, S. H., \& Difazio, S. P. (2009). Extensive pollen flow in two ecologically contrasting populations of Populus trichocarpa. Molecular Ecology, 18(2), 357-373. doi: 10.1111/j.1365-294X.2008.04016.x

Slavov, G. T., \& Zhelev, P. (2010). Salient Biological Features, Systematics, and Genetic Variation of Populus. In Genetics and Genomics of Populus (pp. 15-38). doi: 10.1007/978-1-4419-1541-2

Sork, V. L., Aitken, S. N., Dyer, R. J., Eckert, A. J., Legendre, P., \& Neale, D. B. (2013). Putting the landscape into the genomics of trees: Approaches for understanding local adaptation and population responses to changing climate. Tree Genetics and Genomes, 9(4), 901-911. doi: 10.1007/s11295013-0596-х

Sork, V. L., Squire, K., Gugger, P. F., Steele, S. E., Levy, E. D., \& Eckert, A. J. (2016). Landscape genomic analysis of candidate genes for climate adaptation in a california endemic oak, Quercus lobata. American Journal of Botany, 103(1), 33-46. doi: 10.3732/ajb.1500162

Storfer, A., Patton, A., \& Fraik, A. K. (2018). Navigating the interface between landscape genetics and landscape genomics. Frontiers in Genetics, 9(MAR). doi: 10.3389/fgene.2018.00068

Stucki, S., Orozco-terWengel, P., Forester, B. R., Duruz, S., Colli, L., Masembe, C., ... Joost, S. (2017). High performance computation of landscape genomic models including local indicators of spatial association. Molecular Ecology Resources, 17(5), 1072-1089. doi: 10.1111/1755-0998.12629

Talbot, B., Chen, T. W., Zimmerman, S., Joost, S., Eckert, A. J., Crow, T. M., ... Manel, S. (2017). Combining genotype, phenotype, and environment to infer potential candidate genes. Journal of Heredity, 108(2), 207-216. doi: 10.1093/jhered/esw077

Tiffin, P., \& Ross-Ibarra, J. (2014). Advances and limits of using population genetics to understand local adaptation. Trends in Ecology and Evolution, 29(12), 673-680. doi: 10.1016/j.tree.2014.10.004

van den Wollenberg, A. L. (1977). Redundancy analysis an alternative for canonical correlation analysis. Psychometrika, 42(2), 207-219. doi: 10.1007/BF02294050

Vangestel, C., Eckert, A. J., Wegrzyn, J. L., St. Clair, J. B., \& Neale, D. B. (2018). Linking phenotype, genotype and environment to unravel genetic components underlying cold hardiness in coastal Douglas-fir (Pseudotsuga menziesii var. menziesii). Tree Genetics and Genomes, 14(1). doi: 10.1007/s11295-017-1225-x

Wang, J., Ding, J., Tan, B., Robinson, K. M., Michelson, I. H., Johansson, A., ... Ingvarsson, P. K. (2018). A major locus controls local adaptation and adaptive life history variation in a perennial plant. Genome Biology, 19(1), 72. doi: 10.1186/s13059-018-1444-y

Wang, T., Hamann, A., Spittlehouse, D. L., \& Murdock, T. Q. (2012). ClimateWNA-high-resolution 
spatial climate data for western North America. Journal of Applied Meteorology and Climatology, 51(1), 16-29. doi: 10.1175/JAMC-D-11-043.1

Zhou, L., Bawa, R., \& Holliday, J. A. (2014). Exome resequencing reveals signatures of demographic and adaptive processes across the genome and range of black cottonwood (Populus trichocarpa).

Molecular Ecology, 23(10), 2486-2499. doi: 10.1111/mec.12752

Zhou, X., \& Stephens, M. (2012). Genome-wide efficient mixed model analysis for association studies. Nature Genetics, 44(7), 821-824. doi: 10.1038/ng.2310.

Zhou, X., \& Stephens, M. (2014). Efficient multivariate linear mixed model algorithms for genome-wide association studies. Nature Methods, 11(4), 407-409. doi: 10.1038/nmeth.2848 


\section{Chapter 4. Genome-wide association study of wood anatomical, wood chemistry and morphological traits in Populus trichocarpa}

\subsection{Abstract}

To understand the genetic mechanisms underlying wood anatomy, wood chemistry and morphological traits in Populus trichocarpa, we used 869 unrelated genotypes from a common garden in Clatskanie, OR that were previously collected from across the distribution range in western North America. Using GEMMA mixed model analysis, we tested for the association of 27 phenotypic traits and 19 multitrait combinations with 6.741 million SNPs covering the entire genome. Broad-sense trait heritabilities ranged from 0.117 to 0.477 . As expected, traits were correlated within the trait types anatomical traits were correlated to each other and morphological traits were correlated to each other. Most traits were significantly correlated with geoclimatic variables suggesting the role of climate and geography in shaping the variation of this species. We identified a total of 20 and 33 gene models from single and multitrait GWAS, respectively. Two SNPs from single trait GWAS and 10 SNPs from multitrait GWAS passed a Bonferroni threshold of $7.417 \times 10^{-9}$, leading to the identification of two and eight nearby candidate genes, respectively. We have presented here one of the most comprehensive GWAS analyses for $P$. trichocarpa to date including the first GWAS for wood anatomical traits for this species. We identified important genes related to defense mechanisms and abiotic stress tolerance with wood anatomical and wood chemistry GWAS and genes involved in light and hormone signaling pathways with morphological trait GWAS. The identified genes have great potential for optimizing traits for lignocellulosic biofuel production.

\subsection{Introduction}

It is of increasing interest to identify the molecular variants underlying adaptive and morphological trait variation in plant populations. Loci highlighted by such analyses have great potential for optimizing the trait of interest through genetic engineering or breeding, thereby producing trees with increased productivity, enhanced abiotic stress tolerance, and/or improved quality of end products. Because of their wide geographical distribution and climatic gradients, large effective population sizes, and high genetic variation, forest trees are excellent model systems for understanding local adaptation and the genetic architecture of the complex traits (González-Martínez, Ersoz, Brown, Wheeler, \& Neale, 2006; Ingvarsson, Hvidsten, \& Street, 2016; Neale \& Kremer, 2011; Neale \& Savolainen, 2004; Street \& Ingvarsson, 2011). In this regard, efforts have been made to optimize the ecologically and economically important tree Populus for lignocellulosic biofuel production. Vast amounts of genomic and phenotypic resources are available for the genus. Several large-scale genome-wide association studies have identified the underlying genetic architecture related to morphological, physiological, wood chemistry and disease 
resistance traits (Bdeir et al., 2019; McKown, Klápště, et al., 2014; Muchero et al., 2015; Zhang et al., 2018). Furthermore, the biology of wood formation, cell wall ultrastructure and composition, and cell wall recalcitrance are fairly well studied (Allwright et al., 2016; Du et al., 2016, 2018; Du, Pan, Xu, Li, \& Zhang, 2013; Du et al., 2019; Escamez et al., 2017; Fahrenkrog et al., 2017; Gandla, Martín, \& Jönsson, 2018; Groover, Nieminen, Helariutta, \& Mansfield, 2010; Johnson, Kim, Ralph, \& Mansfield, 2017; Muchero et al., 2015; Porth et al., 2015; Porth, Klápště, et al., 2013; Porth, Ranjan, et al., 2013; Studer et al., 2011; Wegrzyn et al., 2010; Xi, Song, Sun, Shen, \& Li, 2017). However, the genetic architecture underlying wood anatomical traits such as vessel size and density is relatively unknown, despite the importance of these traits for cell wall composition and the overall performance of the tree.

Wood anatomy not only contributes to the structural integrity of the tree, but it is also critical for transport and storage processes (Hietz, Rosner, Hietz-Seifert, \& Wright, 2017; Sperry, 2003). Anatomical structures like vessel size and density are related to cell wall structure and composition and wood density. These traits together affect long-distance axial transport of nutrients and hydraulic conductivity. Lignified cell walls and fibers add strength and living parenchyma cells provide radial transport and storage. There are often trade-offs among vessel properties, wood density and hydraulic conductivity (Preston, Cornwell, \& DeNoyer, 2006). Wood traits are generally heritable (Carlquist, 2012) and serve as useful traits in phylogenetic analyses (Hietz et al., 2017). Radial variation in wood anatomical properties in the stem affects wood functional traits such as hydraulic conductivity and often scales with tree size and leaf characteristics (Lachenbruch, Moore, \& Evans, 2011). Genome-wide association studies of wood anatomy traits undoubtedly complement the current understanding of genetic architecture of other structural and functional traits in Populus trichocarpa and will serve as an important step for optimizing traits suitable for lignocellulosic biofuels. Furthermore, GWAS of morphological traits collected from the same tree and the same time period provide a better understanding of the overall genetic architecture of complex traits.

Nevertheless, the limitation of power due to sample size in detecting the genetic variants associated with complex traits is a major hurdle in trees. Despite the recent revolution in the genomic technology, establishment, management, and intensive phenotyping of large common gardens is expensive and logistically challenging. Furthermore, SNP loci and candidate genes identified thus far explain only a small proportion of the genetic variation in complex traits in general (Visscher et al., 2017). Genes controlling complex traits do not work in isolation, but instead are interconnected in networks of thousands of genes, each of which may contribute incrementally to the variation in complex traits (Boyle, Li, \& Pritchard, 2017). Recently, methods such as multitrait GWAS and meta analyses using summary statistics have become increasing popular due to their role in enhancing the power of GWAS and the identification of potentially pleiotropic loci (Porter \& O'Reilly, 2017). While no raw data 
is required for summary statistics, using raw data for multitrait GWAS can enhance power and lead to discovery of novel associations (Chhetri et al., 2019).

Here we focus on Populus trichocarpa, a targeted species for lignocellulosic biofuel production that has a distribution spanning from northern California to northern British Columbia. Tremendous resources including whole-genome resequencing data, multiple common gardens for association mapping, transcriptome and metabolite data and expression networks are available for this species (Chhetri et al., 2019; Weighill et al., 2019). Here we present a genome-wide association study of wood anatomical traits for the first time for this species. We also present a GWAS for important morphological and wood chemistry traits from the same trees that together with anatomical traits affect overall plant productivity. We complemented the single trait GWAS with multitrait analyses. Since this study is based on data collected from a common garden in Clatskanie, OR this allowed for the direct comparison of GWAS genes identified for the same traits from other plantations (Chhetri et al., 2019; Evans et al., 2014).

\subsection{Methods}

\subsubsection{Phenotypic data collection}

Wood anatomical, wood chemistry and morphological trait data were collected from a field trial of 1100 P. trichocarpa genotypes that was established in Clatskanie, OR in 2009 (Figure 4.1). These genotypes were previously collected from across the natural range of $P$. trichocarpa from northern California to northern British Columbia and were clonally replicated and planted in a randomized block design with three replicates of each genotype at $2 \mathrm{~m} \mathrm{x} 3 \mathrm{~m}$ spacing in the field trial (Evans et al., 2014; Slavov et al., 2012).

In June 2012, 557 trees were sampled for wood anatomical traits. Wood cores of $12 \mathrm{~mm}$ diameter in size were taken from the main trunk of the tree using an increment borer. Free hand cross-sections were made from the previous year's growth ring and fixed in 70\% alcohol. The tissue sections were stained in $1 \%$ Safranin O solution for 30 seconds before preparing the slides for imaging. Images at 100x magnification were taken to sample the early, intermediate and late wood from the growth ring. 100x images were used for measuring vessel density, size and number (Figure 4.2). All images were processed using the software imageJ to extract quantitative measurements - vessel count, density and size (Schindelin, Rueden, Hiner, \& Eliceiri, 2015).

Leaf characteristics were measured for $676-687$ trees (Table 4.1). The first and second fully expanded leaf (counting from the apex) were collected from a branch receiving full sunlight. One of the leaves was used for measuring petiole length and minimum and maximum petiole diameter with a caliper and then scanned using a hand-held scanner. The imageJ software (Schindelin et al., 2015) was used to estimate leaf area, leaf circularity, leaf length, leaf width and leaf perimeter. Dry and wet weights were measured for the same leaf and leaf area and leaf dry weight were used to estimate the specific leaf area 
(SLA). Leaf chlorophyll content (SPAD) was assessed using a SPAD 502 Plus meter (Spectrum Technologies) with an average of 3 replicate measures on a leaf section. The second leaf was used for measuring the abaxial stomatal density. Clear nail polish was applied to the broadest part of the leaf close to the midrib. A clear piece of tape was then used to capture an imprint of the epidermal leaf surface. The slides for the imprints were prepared and the number of stomata in $1 \mathrm{~mm}^{2}$ area in four random microscopic fields at 400x magnification were counted.

\subsubsection{Phenotyping for lignin content and $S / G$ ratio}

Details of the methods for estimating lignin content and the ratio of syringyl:guaiacyl (S/G) lignin monomers are fully described in Muchero et al. (2015). Briefly, 1456 wood samples (919 trees sampled in June and 535 in December of 2012) and 1462 (925 trees sampled in June and 537 in December of 2012) were analyzed for lignin content and $\mathrm{S} / \mathrm{G}$ ratio, respectively from the plantation in Clatskanie, OR. The increment cores were air-dried before estimating lignin content and S/G ratio using pyMBMS analysis.

\subsubsection{Statistical analyses}

To estimate the genetic control of quantitative traits, broad-sense heritability $\left(H^{2}\right)$ was estimated for all traits using the genotypes with replicate clonal measurements using the following formula:

$$
H^{2}=\frac{\sigma_{G}^{2}}{\sigma_{G}^{2}+\sigma_{E}^{2}},
$$

where $\sigma_{G}^{2}$ is genotypic variance due to clonal differences and $\sigma_{E}^{2}$ is environmental variance.

Outliers were removed, and the data were evaluated for normality. Variance components were estimated employing the liner regression model with the lmer and ranef functions of the lme4 package implemented in R. Genotype and the position of the tree (i.e. row and column) and year (for lignin and $\mathrm{S} / \mathrm{G}$ ratio) in the garden was used as a random effect in the model. Error was estimated from the residuals of the model. Genetic correlation between the traits was estimated using the Best Linear Unbiased Predictors (BLUPs) from the same model.

\subsubsection{Genotypic data}

Methods for obtaining genotypic data were as described previously (Chhetri et al., 2019; Evans et al., 2014; Weighill et al., 2018). Briefly, whole genome re-sequencing data was obtained from 1053 trees using Illumina genetic analyzers at the DOE Joint Genome Institute. After removing trees related more closely than first cousins and highly differentiated California trees, 869 trees were left, which were used for all analyses. A genetic relationship matrix was estimated using GEMMA and used as a covariate in the GWAS analyses. Furthermore, SNPs with minor allele frequency $\leq 0.05$ and markers with severe departures from Hardy-Weinberg expectations were removed. 


\subsubsection{Association analysis}

The tests for statistical association were employed using Genome-wide Efficient Mixed Model Association package (GEMMA, Zhou \& Stephens, 2012, 2014). Phenotypic BLUPs, genetic relationship matrix and 6,741,160 SNPs were used for the association test. Single trait GWAS was run for 27 phenotypes (Table 4.1). The tested model was:

$y=W \alpha+x \beta+u+\epsilon$,

where $y$ is an $n$-vector of phenotypic BLUP values, where $n$ is the number of individuals tested; $W$ is an $n \times c$ matrix of covariates; $\alpha$ is a $c$-vector of corresponding coefficients, where $c$ is the number of principal coordinate axes used; $x$ is an $n$-vector of marker genotypes, $\beta$ is the effect size of the marker, $u$ is an n-vector of random effects that includes a relatedness matrix and $\epsilon$ is an $n$-vector of errors.

Multitrait GWAS was run for 19 multitrait sets (Table 4.2). The same procedure was used for selecting trait sets as described in Chhetri et al. (2019). Multitrait association was conducted with GEMMA using the same model as for single trait associations, except $y$ is an $n \times d$ matrix of $d$ phenotypes for $n$ individuals.

\subsubsection{Analyses of association results}

We used a $P$-value cutoff $(\alpha \leq 0.05)$ based on the Bonferroni correction criterion of $7.417 \times 10^{-9}$ and a more liberal $P$-value cutoff of $1 \times 10^{-7}$ to identify suggestive associations. For the purpose of summarizing the results, significant SNPs within $10 \mathrm{~kb}$ of one another were merged into peaks. Gene models that were closest to significant SNPs were identified based on v3 of the P. trichocarpa genome. Annotation information including gene expression level in different plant tissues and annotation of putative gene function was obtained from Phytozome (Goodstein et al., 2012). Percentage of variance explained (PVE) by SNPs was estimated using the formula in Shim et al. (2015).

\subsection{Results}

\subsubsection{Heritabilities of wood anatomy, chemistry, and morphology traits}

Overall the broad-sense heritabilities estimated for wood anatomy, chemistry and morphology related traits in this study were low to moderate (Table 4.1). Broad-sense heritabilities for wood anatomy and wood chemistry traits ranged from 0.114 for late wood vessel area to 0.464 for S/G ratio. Late wood anatomy (late wood vessel count, vessel area and vessel size) and lignin content had low heritabilities compared to other wood traits. For morphology related traits, broad-sense heritabilities ranged from 0.122 for specific leaf area to 0.477 for stomatal density.

Narrow sense heritabilities (SNP chip heritabilities) for wood anatomy and chemistry traits ranged from 0.199 for early wood vessel size to 0.708 for $\mathrm{S} / \mathrm{G}$ ratio whereas for morphology related traits it ranged from 0.038 for specific leaf area to 0.965 for tree height (Table 4.1). Although there was no correlation between broad-sense and SNP chip heritabilities ( $\mathrm{r}$ not shown), low SNP chip heritability was 
generally reflective of low broad sense heritability and there was a significant correlation between the sample size (number of genotypes used for GWAS) and SNP chip heritability values $(\mathrm{r}=0.441, P=0.021)$ for the traits measured in this study (Table 4.1)

\subsubsection{Genetic correlation of phenotypic traits within and between the common gardens}

As expected, most morphological traits measured in the Clatskanie common garden were correlated to each other - leaf traits such as leaf area, leaf length, leaf dry weight, leaf wet weight, petiole diameter and length, leaf perimeter and leaf aspect ratio were highly significantly correlated to each other and SPAD and stomatal density had low, but significant correlations with most leaf traits (Table 4.3). Similarly, most wood traits were correlated to each other. S/G ratio was significantly correlated with lignin content. Wood anatomy traits such as vessel area and size and vessel count within each of the growth ring regions (early, intermediate or late wood indicating a different maturation stages) were correlated to each other. Wood anatomy traits were also significantly correlated to each other across growth ring areas (Table 4.3). Furthermore, some morphological traits such as tree height and diameter

were significantly (although weakly) correlated with wood traits such as S/G ratio, early wood vessel size and area, intermediate wood vessel size and count, and late wood vessel size and count (Table 4.3).

Genetic correlation of the same phenotypic traits measured between the Clatskanie and Corvallis common gardens showed that most traits were weakly, but significantly correlated to each other (Table 4.4). Pairwise genetic correlations of tree height $(\mathrm{r}=0.436, P<0.001)$ and stomatal density $(0.345$, $P<0.001)$ between the sites showed the strongest correlations among all traits compared (Table 4.4).

\subsubsection{Phenotypic trait correlations with climate variables}

As seen for the phenotypic traits in Corvallis common garden (Chhetri et al., 2019), most phenotypic traits had significant correlations with latitude, and therefore the correlations between the phenotypic traits and other geoclimate variables cannot easily be discerned. Nevertheless, most morphological traits had significant (although weak) correlations with most geoclimatic variables (Table 4.5). Similarly, wood traits such as S/G ratio and late wood vessel area had significant (although weak) correlations with most geoclimatic variables (Table 4.5).

\subsubsection{Genes identified from single trait and multitrait GWAS}

We performed single trait GWAS with 6.741 million SNPs for 27 morphological and wood anatomical and wood chemistry traits. Only two SNPs passed the Bonferroni correction threshold of $\mathrm{P}<7.417 \times 10^{-9}$ (Table 4.1). However, we identified a total of 77 SNPs that passed suggestive association $P$-value cutoff of $1 \times 10^{-7}$ (Table 4.1, Figure 4.3, Supplementary Figure S4.1). These associated SNPs belonged to 12 separate SNP peaks and were within or close to 20 P. trichocarpa gene models (Table 4.6). PVE for significant SNPs ranged from $3.30 \%$ to $5.72 \%$. 
Multitrait GWAS performed on 19 sets of traits identified 10 SNPs that passed the Bonferroni correction $P$-value cutoff and 47 SNPs that passed the suggestive association $P$-value cutoff of $1 \times 10^{-7}$ and 9 SNPs passed Bonferroni correction threshold of $\mathrm{P}<7.417 \times 10^{-9}$ (Table 4.2, Figure 4.4, Supplementary Figure S4.2). These SNPs belonged to 31 separate SNP peaks and were within or close to $33 P$. trichocarpa gene models (Table 4.7). PVE of these SNPs ranged from $0.0003 \%$ to $6.32 \%$ for the individual traits comprising the multitrait set (large table, data not shown). One gene overlapped between single and multitrait GWAS.

\subsubsection{Genes identified for wood anatomical and wood chemistry traits}

Out of the single trait GWAS for 11 wood anatomical and wood chemistry traits, we identified a total of 5 gene models belonging to 4 traits. A total of 23 gene models were identified for 13 multitrait sets related to wood traits.

\subsection{Discussion}

\subsubsection{Patterns of genetic variation}

Broad-sense heritabilities for most traits in this study ranged from $0.2-0.4$, suggesting that the traits were under moderate genetic control. Heritability estimates for morphological traits were comparable to other similar studies (Chhetri et al., 2019; Evans et al., 2014; Mckown et al., 2014). However, some wood related traits and SLA had low heritabilities $(<0.2)$, which may be reflective of relatively small sample size (although this is not the case for lignin content) compared to morphological traits. This might also suggest that the environment had a strong influence on these traits, but it seems unlikely that wood anatomical traits would be more susceptible to environmental effects than morphological traits. One possible reason is the variation in micro-environment in the common garden that might affect the growth ring pattern in trees, so that there is more error in estimating the wood anatomy traits from small wood samples. In any case, the results for these low heritability traits should be interpreted with caution.

As reported in previous studies geography has a major role in shaping adaptive trait variation in P. trichocarpa. Correlation of these adaptive traits with latitude obscures the actual between-trait and trait-climate relationships (Chhetri et al., 2019). Most morphological traits such as tree height and leaf characteristics had strong significant correlations with latitude compared to previous studies (Chhetri et al., 2019; Mckown et al., 2014). In contrast to Chhetri et al. (2019), tree height including diameter at breast height had a very low or no correlation with leaf traits as in McKown et al. (2014). However, tree height and diameter had significant negative correlations with wood chemistry (S/G ratio) and intermediate and late wood vessel counts and significant positive correlations with vessel size of all maturation stages (early, intermediate and late woods). However, vessel density was positively correlated with latitude and vessel size was negatively correlated with latitude. It is therefore difficult to deconvolute 
the indirect effects of latitudinal variation from the direct effects of wood anatomy on productivity in this study.

Further corroboration for the effects of environment shaping these traits can be gleaned from comparing the common gardens. Genetic correlation of the directly comparable traits between the Clatskanie and Corvallis common garden showed very low or no correlations except for stomatal density and tree height (Table 4.4). Furthermore, different loci seem to be controlling phenotypic traits in the two gardens, based on a complete lack of overlap in significant loci discovered by GWAS in the Corvallis, OR common garden (Chhetri et al., 2019). This might be due to differential effects of local environments on phenotypes in each of the respective common gardens.

As temperature decreases, and moisture and precipitation increase with latitude, relationships of the phenotypic traits with the climate variables corroborates the trait relationships explained above. S/G ratio and leaf characteristics including leaf dry weight and petiole length and diameter had significant positive correlation with latitude, mean annual precipitation and relative humidity, but significant negative correlation with mean annual temperature. Similarly, abaxial stomatal density was negatively correlated with precipitation (Table 4.5). Taken together, southern trees had higher S/G ratio, larger but less dense vessels, smaller leaves with higher abaxial stomatal density and low chlorophyll content, and larger tree height and diameter.

$\mathrm{S} / \mathrm{G}$ ratio was significantly correlated with lignin content and had significant negative correlations with tree height and diameter. S/G ratio increases with latitude and decreases with temperature, but late wood vessel area (similar to the relationship of height and diameter with latitude and temperature) decreases with latitude and increases with temperature.

\subsubsection{GWAS genes}

The identification of genomic variants (and associated gene models) controlling morphological and anatomical and wood chemistry traits in this study suggests that these traits are under polygenic control as expected. While we found none to only a few significantly associated or suggestive SNPs for most traits we studied here, we think that this is mainly due to lack of power due to sample size although this is one of the most comprehensive GWAS analyses to date in terms of the number of SNPs used. We did not find any gene model controlling more than one trait from our single trait GWAS analyses, but we identified 33 gene models with potential pleiotropic effects controlling sets of morphological, wood anatomical and wood chemistry traits (Table 4.7).

The GWAS analysis for wood anatomical traits we reported here is the first such study in Populus. We identified 4 gene models from single trait GWAS and 7 gene models from multitrait sets that include only wood anatomical traits. We found one additional gene model from single trait and 15 additional gene models from multitrait GWAS that included wood chemistry traits, lignin and S/G ratio 
independently or in combination with wood anatomical traits. None of the genes we identified here has been reported in earlier studies.

\subsubsection{Gene models detected using single trait and multitrait GWAS}

Using single and multitrait GWAS for wood anatomy and chemistry and morphology related traits we identified genes implicated in stress responses, defense mechanisms, growth and development, amino acid and hormone transporters, involvement in RNA interference (RNAi) and micro RNA (miRNA) pathways, signaling pathways, cellular and developmental processes, and enzyme inhibitors. However, we also identified a few genes with unknown functions.

The two GWAS hits from the single trait GWAS that passed the Bonferroni threshold of $\mathrm{P}<7.417 \times 10^{-9}$ were both from the GWAS with stomatal density (Figures $4.3 \mathrm{k} \& 1$ ). One of these SNPs with $P$-value $2.77 \times 10^{-9}$ was within the gene model, Potri.015G117500 that encodes peptide-Ofucosyltransferase. The gene is highly expressed in the first fully expanded leaf, early and mid-spring stems, normal root, root treated with ammonia and nitrate; low expression in early and late female development organs and late fall stems and moderate expression in apical bud, immature and young leaves, early male development, fully open, predormant, early and late dormant and fully open bud, early winter stem, root tip, mid male development, stem treated with ammonia, nitrate and urea, stem node and internode (Figure 4.5). In Arabidopsis O-fucosyltransferase affects DELLA and associated regulators such as phytochrome-interacting-factor3 (PIF3) and PIF4 in the barasinosteroid- and light-signaling pathways (Zentella et al., 2017). DELLA proteins are important integrators of multiple signaling pathways in flowering plants. DELLA are repressors of phytohormone (GA) signaling, master growth repressors that restrict plant growth by affecting genes in cell division, expansion and differentiation (Zentella et al., 2007). In Arabidopsis O-fucosyltransferase modifies DELLA protein RGA (repressor of gal-3). The GA-deficient mutant gal-3 is a nongerminating, extreme dwarf that flowers late and produces male-sterile flowers. This protein is also known to be involved in cell-to-cell adhesion (deficiency in pectin biosynthesis pathway leading to loss of cell adhesion) together with GAUT8 (QUASIMODO1, see below) in Arabidopsis (Verger, Chabout, Gineau, \& Mouille, 2016). McKown, Guy, et al. (2014) identified a similar gene, Potri.004G059000, encoding BRASSINOSTEROIDINSENSITIVE 2 for stomatal density based on the GWAS on 34K SNP array data for $464 P$. trichocarpa trees. BRASSINOSTEROID-INSENSITIVE 2 potentially affects the brassinosteroid signaling pathway and the regulation of stomatal development (Gudesblat et al., 2012; Khan et al., 2013). The other top GWAS hit for stomatal density was within a gene model Potri.015G117300 of unknown function. The gene is highly expressed in early and mid-spring stems, stem treated with ammonia, nitrate and urea, root tip, the first fully expanded and young leaves and fully open bud (Figure 4.5) 
We identified a total of 8 genes based on the 10 SNPs that passed Bonferroni threshold in the multitrait GWAS, of which 4 genes were detected for the leaf morphology multitrait, (leaf area, leaf dry weight, leaf length and leaf width) and 4 genes were detected for 4 wood anatomy and wood chemistry related multitrat GWASs (Figures $4.4 \& 4.7$ ). The gene model near the top GWAS hit (SNP Pvalue $=3.62 \times 10^{-13}$ ) for leaf morphology multitrait was Potri.010G032600, which encodes a small subunit (40S) ribosomal protein S11. Ribosomal proteins are involved in gene regulation, cellular functions and development mechanisms, apoptosis and aging (Bhavsar, Makley, \& Tsonis, 2010). The SNP is 9.5kb downstream of this gene. No expression data is available for this gene (Figure 4.6). There are at least 249 genes encoding 80 ribosomal proteins in Arabidopsis (Barakat et al., 2001) and are known to have effects in growth and development (Devis, Firth, Liang, \& Byrne, 2015).

With leaf morphology multitrait we also identified the gene Potri.019G067300, 10.5kb downstream of a significant SNP $\left(P\right.$-value $\left.=1.9 \times 10^{-8}\right)$ that encodes a protein related to galactosyltransferase-8 (GAUT8). A gene with a similar function, Potri.004G111000, that encodes GAUT9 was detected for the same multitrait GWAS from the Corvallis common garden (Chhetri et al., 2019). GAUT8 and GAUT9 belonged to the same clade B1 in a phylogenetic tree based on the GAUT protein family of Arabidopsis thaliana and P. trichocarpa (Biswal et al., 2018). GAUT9 affects leaf size in Populus deltoides (Chhetri et al., 2019) whereas GAUT8 (also known as QUASIMODO1) has high expression in stems in Arabidopsis (Caffall, Pattathil, Phillips, Hahn, \& Mohnen, 2009) and is thought to be involved in cell wall pectic homogalacturonan (HG) and xylan biosynthesis (Orfila et al., 2005; Verger et al., 2016). Genes encoding glycosyltransferases, a large family of enzymes mainly involved in biosynthesis of polysaccharides and glycoproteins in the plant cell wall (Hansen, Harholt, Oikawa, \& Scheller, 2012), were also detected in single trait GWAS for stomatal density and leaf aspect ratio in this study (Table $4.6 \& 4.7$ ).

The gene model Potri.006G275800 was found $8.7 \mathrm{~kb}$ downstream of the top GWAS hit for wood multitrait 1 (vessel area and vessel count for early, intermediate and late wood, and lignin and S/G ratio; SNP $P$-value $=1.03 \times 10^{-9}$, Table 4.4) and for the top two GWAS hits for wood anatomy multitrait 4 (intermediate wood vessel area and count, both SNPs with $P$-value $=3.99 \times 10^{-8}$ ). The gene encodes leucine rich repeat-containing protein that has a function in defense. A gene with a similar annotation (Potri.012G028700) was detected for leaf morphology multitrait ( ${ }^{\text {th }}$ top GWAS hit for leaf morphology multitrait, SNP $P$-value $=5 \times 10^{-9}$, gene found $1.3 \mathrm{~kb}$ upstream of the SNP) as well (Table 4.4). This suggests functional relationships among wood anatomical, wood chemistry and morphological traits.

The gene model Potri.019G037900 was found 1.2kb upstream of the second best GWAS hit for wood multitrait 5 (intermediate wood vessel size, lignin and S/G ratio; SNP $P$-value $=1.77 \times 10^{-9}$ ) which encodes for double-stranded-RNA-binding proteins. In Arabiodopsis, the biogenesis of small RNA 
(miRNA and tasiRNA) requires the involvement of DOUBLE-STRANDED RNA BINDING (DRB) proteins (Eamens, Kim, Curtin, \& Waterhouse, 2012; Eamens, Smith, Curtin, Wang, \& Waterhouse, 2009). Small RNAs are involved in regulating the expression of genes related to growth and development. The same gene Potri.019G037900 (and the SNP) was detected in single trait GWAS for intermediate wood vessel size as well, but the SNP had a weaker association $\left(P\right.$-value $\left.=8.44 \times 10^{-9}\right)$.

The gene model Potri.004G183900 was found 4.6kb upstream of the top GWAS hit for wood anatomy multitrait 5 (late wood vessel area and count, SNP $P$-value $=5.91 \times 10^{-9}$ ) which encodes a receptor like kinase (RLK), serine/threonine protein kinase. RLKs play major roles in defense mechanisms (e.g. disease resistance) and development functions in plants (Afzal, Wood, \& Lightfoot, 2008). Genes encoding L-type RLKs are involved in resistance to the fungal pathogen Sphaerulina musiva in $P$. trichocarpa (Muchero et al., 2018). Wood anatomy is related to lignin composition in plants and lignin biosynthesis genes are involved in defense mechanisms (Moura, Bonine, de Oliveira Fernandes Viana, Dornelas, \& Mazzafera, 2010; Xie et al., 2018).

The gene model Potri.017G090200 was found 1.8kb upstream of the $2^{\text {nd }}$ top GWAS hit for wood multitrait 1 , (SNP $P$-value $\left.=7.32 \times 10^{-9}\right)$ and encodes calmodulin-binding protein. Calmodulin $(\mathrm{CaM})$ and calmodulin-like proteins (CMLs) are one of the three main families of calcium $\left(\mathrm{CA}^{2+}\right)$ sensor proteins in plants (Ranty et al., 2016). Calmodulin (CaM) and calmodulin-like proteins (CMLs) are well characterized in plants and are involved in regulating plant responses to abiotic stresses (Zeng et al., 2015).

One of the calmodulin binding proteins, EF-hand motif that is involved in cell signaling, developmental processes and biotic and abiotic responses was detected in the multitrait GWAS from Corvallis, OR plantation that included carbon isotope, leaf area and stomatal density in the multitrait set (Chhetri et al., 2019). Here we identified a gene with similar role controlling wood anatomy traits. Wood anatomy traits have potential functional relationships with carbon isotope, leaf area and stomatal density. 4.5.4 Comparison with previous studies

We compared our GWAS results with the GWAS from the previous studies for the same traits in P. trichocarpa (Chhetri et al., 2019; Evans et al., 2014; McKown, Klápště, et al., 2014; Porth, Ranjan, et al., 2013; Wegrzyn et al., 2010), but we did not find any overlapping gene models. This may be due to a) differential environmental effects on the traits due to plantation sites, b) traits measured in different seasons of the year and different years were subjected to varied environmental pressures, c) variability in the microenvironment within the sites as reflected by the different broad-sense heritability values, d) differences in the developmental stages of the traits across the studies and e) difference in the genotypes and the sequence data types (e.g. SNP array vs whole genome sequence data). Furthermore, we were not able to replicate any significant SNP hits or the gene models for the same traits from another $P$. 
trichocarpa common garden in Corvallis, OR in our study, although several single traits such as stomatal density, petiole diameter and tree height and the multitrait set containing leaf area, leaf dry weight, leaf length and leaf width were common between the two common gardens [Clatskanie, OR (this study) and Corvallis, OR] and measurements were performed using the same methods. This might very well reflect the differential influence of the environment on genotypes in the two populations. Nevertheless, we believe that our comprehensive GWAS study with genome-wide sequencing data highlights the underlying genes controlling adaptive traits in P. trichocarpa and complements the findings from other similar studies. Moreover, we have reported here genes underlying complex wood anatomy traits in Populus for the first time.

\subsection{Conclusion}

We presented here the first comprehensive GWAS for wood anatomical traits in Populus that provides an insight into the type of genes controlling structural and functional properties important for plant development, function and stress tolerance. We complemented this with GWAS for important wood chemistry and morphological traits that have functional relationships with wood anatomical traits. As shown in previous $P$. trichocarpa GWAS study in another common garden (Chhetri et al. 2019), we achieved more power with multitrait GWAS in this study as well. Furthermore, the multitrait sets formed based on the genetic correlations and the functional relationship of the traits within and among the wood anatomical, wood chemistry and morphological traits provided insight into pleiotropic genes controlling these traits. Some of the genes we identified in this study had no known functions which might be interesting to explore further. Genes identified here, especially the genes controlling wood anatomical traits, can be good targets for biotechnology experiment for optimizing wood traits for biofuel production. However, as is the case with most GWAS studies, despite the use of largescale whole genome resequencing data and much broader sampling compared to most of the previous similar studies and with the use of multitrait GWAS, a very small percent of the variation in the traits were explained by the significant SNPs. This warrants a large and homogeneous sampling from across the range of distribution for future studies. Moreover, none of the genetic variants detected in the GWAS from another common garden was duplicated in this study. This may be because of the differential effect of environment between the common gardens, which is somewhat reflected by very low correlation of the traits between the two common gardens. Furthermore, additional variation due to the difference in the timing (different years) of data collection might have influenced the GWAS study. 


\subsection{Tables and figures}

Table 4.1 Broad-sense heritability estimates $\left(H^{2}\right)$ and the number of SNP-trait associations for wood chemistry and anatomical and morphological traits in P. trichocarpa.

\begin{tabular}{|c|c|c|c|c|c|}
\hline Trait & $H^{2}$ & Genotypes & Total trees ${ }^{\mathrm{a}}$ & $\begin{array}{l}\text { SNPs } \\
<1 \times 10^{-7 b}\end{array}$ & Chip_ $H^{2}(+/-\mathrm{CI})^{\mathrm{c}}$ \\
\hline \multicolumn{6}{|l|}{ Wood anatomy and chemistry } \\
\hline Early wood vessel area & 0.335 & 489 & $548(59)$ & 0 & $0.542(+/-0.545)$ \\
\hline Early wood vessel count & 0.38 & 489 & $548(59)$ & 1 & $0.611(+/-0.582)$ \\
\hline Early wood vessel size & 0.392 & 489 & $548(59)$ & 0 & $0.199(+/-0.325)$ \\
\hline Intermediate wood vessel area & 0.196 & 570 & $633(63)$ & 0 & $0.475(+/-0.300)$ \\
\hline Intermediate wood vessel count & 0.233 & 570 & $633(63)$ & 0 & $0.562(+/-0.484)$ \\
\hline Intermediate wood vessel size & 0.218 & 570 & $633(63)$ & 2 & $0.301(+/-0.406)$ \\
\hline Late wood vessel area & 0.114 & 557 & $636(79)$ & 0 & $0.373(+/-0.359)$ \\
\hline Late wood vessel count & 0.146 & 557 & $636(79)$ & 0 & $0.327(+/-0.388)$ \\
\hline Late wood vessel size & 0.22 & 557 & $636(79)$ & 1 & $0.373(+/-0.359)$ \\
\hline Lignin content & 0.177 & 830 & $1456(492)$ & 0 & $0.36(+/-0.355)$ \\
\hline $\mathrm{S} / \mathrm{G}$ ratio & 0.464 & 831 & $1462(494)$ & 1 & $0.708(+/-0.312)$ \\
\hline \multicolumn{6}{|l|}{ Morphology } \\
\hline Diameter (breast height) & 0.227 & 869 & $2438(860)$ & 9 & $0.929(+/-0.278)$ \\
\hline Height & 0.294 & 869 & $2438(860)$ & 0 & $0.965(+/-0.257)$ \\
\hline Leaf area & 0.434 & 676 & 813 (137) & 0 & $0.87(+/-0.349)$ \\
\hline Leaf aspect ratio & 0.251 & 676 & 813 (137) & 2 & $0.439(+/-0.320)$ \\
\hline Leaf circularity & 0.285 & 676 & 813 (137) & 6 & $0.313(+/-0.318)$ \\
\hline Leaf dry weight & 0.452 & 685 & $836(151)$ & 18 & $0.99(+/-0.325)$ \\
\hline Leaf length & 0.477 & 676 & 813 (137) & 0 & $0.679(+/-0.320)$ \\
\hline Leaf perimeter & 0.459 & 676 & 813 (137) & 0 & $0.786(+/-0.357)$ \\
\hline Leaf wet weight & 0.393 & 687 & $842(155)$ & 26 & $0.985(+/-0.351)$ \\
\hline Leaf width & 0.386 & 676 & 813 (137) & 0 & $0.905(+/-0.355)$ \\
\hline
\end{tabular}




\begin{tabular}{llrlrl}
\hline \multirow{2}{*}{ Trait } & \multicolumn{3}{c}{ SNPs } \\
\hline Maximum petiole diameter & 0.426 & 687 & $840(153)$ & & \\
Minimum petiole diameter & 0.249 & 683 & $834(151)$ & & $0.928(+/-0.323)$ \\
Petiole length & 0.397 & 685 & $839(154)$ & 0 & $0.843(+/-0.345)$ \\
SPAD & 0.214 & 687 & $843(156)$ & 0 & $0.987(+/-0.335)$ \\
Specific leaf area & 0.122 & 667 & $797(130)$ & 0 & $0.232(+/-0.282)$ \\
Stomatal density & 0.477 & 721 & $884(163)$ & 0 & $0.038(+/-0.318)$ \\
\hline
\end{tabular}

${ }^{a}$ Number of genotypes with replicates in parentheses

${ }^{b}$ Numbers in parentheses indicate significant $P$-value that passed Bonferroni correction threshold of $P<7.417 \times 10^{-9}$.

${ }^{\mathrm{c}}$ Mean Chip Heritability values for phenotypes with confidence interval (CI)

Note: All RLRTpvalues were highly significant except for late wood vessel area, late wood vessel count and specific leaf area, which was not significant. 
Table 4.2 List of traits used for multitrait associations and significant SNPs identified in P. trichocarpa.

\begin{tabular}{|c|c|c|c|c|}
\hline Trait combination & Abbreviation & Trait name & PopN $^{\mathrm{a}}$ & $\begin{array}{l}\text { SNPs } \\
<1 \times 10^{-7 b c}\end{array}$ \\
\hline $\begin{array}{l}\text { Diameter (breast height), height, lignin } \\
\text { content, S/Gratio }\end{array}$ & DB_HT_LI_SG & $\begin{array}{l}\text { morphology and wood } \\
\text { chemisty trait } 1\end{array}$ & 830 & 0 \\
\hline $\begin{array}{l}\text { Diameter (breast height), lignin content, } \mathrm{S} / \mathrm{G} \\
\text { ratio, early wood vessel area }\end{array}$ & DB_LI_SG_EWva & $\begin{array}{l}\text { morphology and wood } \\
\text { multitrait } 1\end{array}$ & 485 & 0 \\
\hline $\begin{array}{l}\text { Early wood vessel area, early wood vessel } \\
\text { count }\end{array}$ & EWva_EWvc & $\begin{array}{l}\text { wood anatomy } \\
\text { multitrait } 1\end{array}$ & 489 & 3 \\
\hline $\begin{array}{l}\text { Early wood vessel area, early wood vessel } \\
\text { count, intermediate wood vessel area, } \\
\text { intermediate wood vessel count, lignin } \\
\text { content, late wood vessel area, late wood } \\
\text { vessel count, S/G ratio }\end{array}$ & $\begin{array}{l}\text { EWva_EWvc_IWva_IW } \\
\text { vc_LI_LWva_LWvc_SG }\end{array}$ & wood multitrait 1 & 407 & $7(2)$ \\
\hline $\begin{array}{l}\text { Early wood vessel area, early wood vessel } \\
\text { count, intermediate wood vessel area, } \\
\text { intermediate wood vessel count, late wood } \\
\text { vessel area, late wood vessel count }\end{array}$ & $\begin{array}{l}\text { EWva_EWvc_IWva_IW } \\
\text { vc_LWva_LWvc }\end{array}$ & $\begin{array}{l}\text { wood anatomy } \\
\text { multitrait } 1\end{array}$ & 411 & 2 \\
\hline $\begin{array}{l}\text { Early wood vessel area, intermediate wood } \\
\text { vessel area, late wood vessel area }\end{array}$ & EWva_IWva_LWva & $\begin{array}{l}\text { wood anatomy } \\
\text { multitrait } 2\end{array}$ & 411 & 0 \\
\hline $\begin{array}{l}\text { Early wood vessel area, leaf area, stomatal } \\
\text { density }\end{array}$ & EWva_LA_SD & $\begin{array}{l}\text { morphology and wood } \\
\text { anatomy multitrait } 1\end{array}$ & 425 & 5 \\
\hline $\begin{array}{l}\text { Early wood vessel area, lignin content, S/G } \\
\text { ratio }\end{array}$ & EWva_LI_SG & wood multitrait 2 & 485 & 0 \\
\hline $\begin{array}{l}\text { Early wood vessel count, intermediate wood } \\
\text { vessel count, late wood vessel count }\end{array}$ & EWvc_IWvc_LWvc & $\begin{array}{l}\text { wood anatomy } \\
\text { multitrait } 2\end{array}$ & 411 & 1 \\
\hline $\begin{array}{l}\text { Early wood vessel count, lignin content, } S / G \\
\text { ratio }\end{array}$ & EWvc_LI_SG & wood multitrait 3 & 485 & 0 \\
\hline $\begin{array}{l}\text { Early wood vessel size, intermediate wood } \\
\text { vessel size, lignin content, } S / G \text { ratio, late } \\
\text { wood vessel size }\end{array}$ & $\begin{array}{l}\text { EWvs_IWvs_LI_SG_L } \\
\text { Wvs }\end{array}$ & wood multitrait 4 & 407 & 3 \\
\hline $\begin{array}{l}\text { Early wood vessel size, intermediate wood } \\
\text { vessel size, late wood vessel size }\end{array}$ & EWvs_IWvs_LWvs & $\begin{array}{l}\text { wood anatomy } \\
\text { multitrait } 3\end{array}$ & 411 & 0 \\
\hline $\begin{array}{l}\text { Intermediate wood vessel size, intermediate } \\
\text { wood vessel count }\end{array}$ & IWva_IWvc & $\begin{array}{l}\text { wood anatomy } \\
\text { multitrait } 4\end{array}$ & 570 & $3(1)$ \\
\hline $\begin{array}{l}\text { Intermediate wood vessel size, lignin content, } \\
\text { S/Gratio }\end{array}$ & IWvs_LI_SG & wood multitrait 5 & 566 & $2(1)$ \\
\hline $\begin{array}{l}\text { Leaf area, leaf dry weight, leaf length, lead } \\
\text { width }\end{array}$ & LA_LD_LL_LW & $\begin{array}{l}\text { leaf morphology } \\
\text { multitrait }\end{array}$ & 674 & $9(4)$ \\
\hline $\begin{array}{l}\text { Lignin content, late wood vessel size, } S / G \\
\text { ratio }\end{array}$ & LI_LWvs_SG & wood multitrait 6 & 552 & 4 \\
\hline Lignin content, $\mathrm{S} / \mathrm{G}$ ratio & LI_SG & $\begin{array}{l}\text { wood chemistry } \\
\text { multitrait }\end{array}$ & 830 & 2 \\
\hline $\begin{array}{l}\text { Lignin content, specific leaf area, stomatal } \\
\text { density, early wood vessel area }\end{array}$ & LI_SL_SD_EWva & $\begin{array}{l}\text { morphology and wood } \\
\text { multitrait } 2\end{array}$ & 415 & 2 \\
\hline $\begin{array}{l}\text { Late wood vessel area, late wood vessel } \\
\text { count }\end{array}$ & LWva_LWvc & $\begin{array}{l}\text { wood anatomy } \\
\text { multitrait } 5\end{array}$ & 557 & $3(1)$ \\
\hline
\end{tabular}


Table 4.3 Pairwise correlation (r) of P. trichocarpa wood chemistry, anatomical and morphological traits in Clatskanie, OR. Values greater than 0.14 or less than -0.14 are significant based on the Bonferroni correction criteria at $5 \%$ significance level.

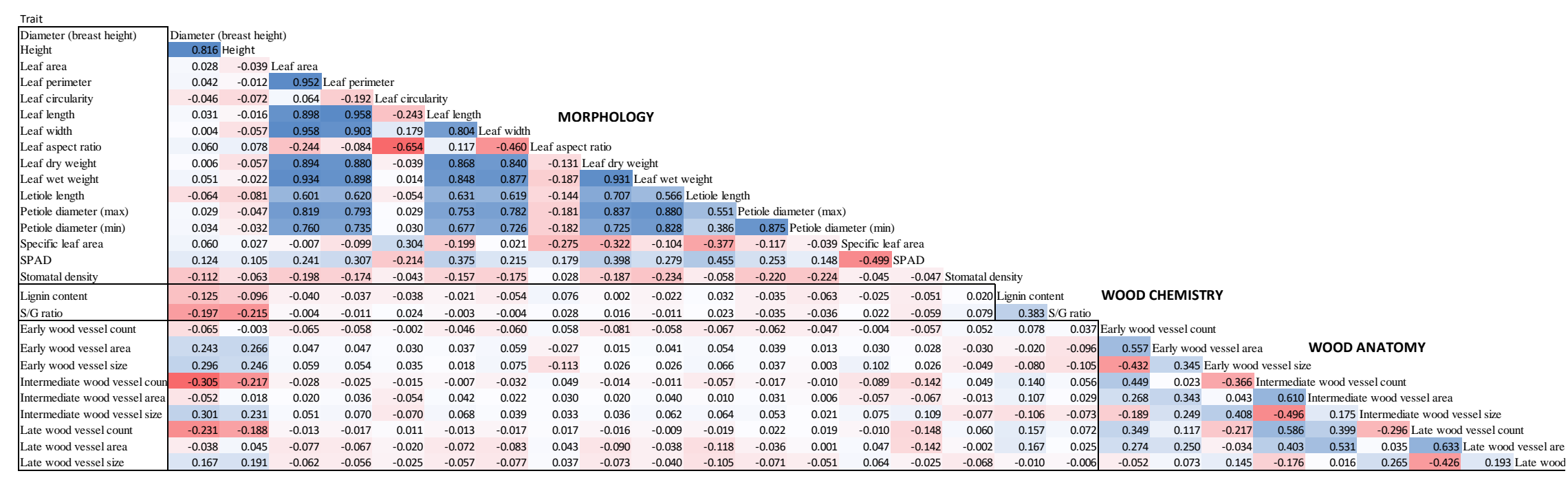


Table 4.4 Pearson correlation (r) of P. trichocarpa wood chemistry, anatomical and morpholgical traits in Clatskanie, OR with morphological and physiological traits in Corvallis, OR. Values greater than 0.14 or less than -0.14 are significant based on the Bonferroni correction criteria at $5 \%$ significance level.

\begin{tabular}{|c|c|c|c|c|c|c|c|c|c|c|c|c|c|c|c|c|c|c|}
\hline & \multicolumn{18}{|c|}{ CORVALLIS } \\
\hline \multirow{28}{*}{ 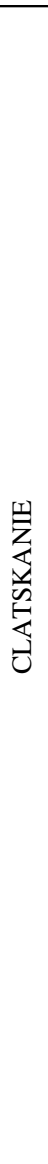 } & Traits & $\begin{array}{l}\text { Water } \\
\text { potential }\end{array}$ & $\begin{array}{l}\text { Stomatal } \\
\text { density }\end{array}$ & SPAD & $\begin{array}{l}\text { Petiole } \\
\text { length }\end{array}$ & $\begin{array}{l}\begin{array}{l}\text { Petiole } \\
\text { diameter } \\
(\max )\end{array} \\
\end{array}$ & $\begin{array}{l}\text { Specific } \\
\text { leaf area }\end{array}$ & $\begin{array}{c}\text { Leaf } \\
\text { area }\end{array}$ & $\begin{array}{l}\text { Leaf } \\
\text { perimeter }\end{array}$ & $\begin{array}{l}\text { Leaf } \\
\text { length }\end{array}$ & $\begin{array}{l}\text { Leaf } \\
\text { width }\end{array}$ & $\begin{array}{l}\text { Leaf } \\
\text { feret }\end{array}$ & $\begin{array}{l}\text { Leaf } \\
\text { aspect } \\
\text { ratio }\end{array}$ & $\begin{array}{l}\text { Leaf dry } \\
\text { weight }\end{array}$ & $\begin{array}{l}\text { Carbon } \\
\text { isotope }\end{array}$ & $\begin{array}{l}\text { Bud } \\
\text { flush }\end{array}$ & Bud set & Height \\
\hline & Diameter (breast height) & 0.09 & -0.09 & -0.07 & 0.21 & 0.17 & $\begin{array}{ll}7 & 0.15\end{array}$ & 0.26 & 0.28 & 0.26 & 0.24 & 0.25 & -0.09 & 0.18 & -0.01 & 0.23 & -0.42 & 20.38 \\
\hline & Height & 0.13 & -0.06 & -0.05 & 0.23 & 0.15 & 0.14 & 0.25 & 0.25 & 0.22 & 0.26 & 0.22 & -0.15 & 0.17 & 0.00 & 0.25 & -0.45 & 0.44 \\
\hline & Leaf area & -0.01 & -0.12 & 0.04 & 0.05 & 0.09 & -0.17 & 0.10 & 0.08 & 0.10 & 0.07 & 0.09 & 0.02 & 0.14 & 0.03 & -0.15 & 0.26 & -0.15 \\
\hline & Leaf perimeter & -0.01 & -0.12 & 0.07 & 0.08 & 0.11 & -0.18 & 0.12 & 0.11 & 0.14 & 0.08 & 0.13 & 0.05 & 0.16 & 0.05 & -0.12 & 0.26 & -0.13 \\
\hline & Leaf circularity & 0.01 & 0.04 & -0.06 & -0.10 & -0.08 & 0.04 & -0.05 & -0.09 & -0.10 & -0.02 & -0.10 & -0.05 & -0.05 & -0.04 & -0.13 & 0.03 & -0.09 \\
\hline & Leaf length & -0.03 & -0.12 & 0.07 & 0.07 & 0.11 & -0.18 & 0.11 & 0.11 & 0.15 & 0.06 & 0.14 & 0.07 & 0.15 & 0.05 & -0.12 & 0.26 & -0.12 \\
\hline & Leaf width & 0.00 & -0.08 & 0.04 & 0.01 & 0.06 & -0.15 & 0.06 & 0.04 & 0.05 & 0.05 & 0.04 & 0.00 & 0.11 & 0.02 & -0.18 & 0.29 & -0.19 \\
\hline & Leaf aspect ratio & -0.02 & -0.07 & 0.06 & 0.08 & 0.09 & -0.01 & 0.09 & 0.13 & 0.17 & 0.03 & 0.17 & 0.11 & 0.06 & 0.05 & 0.15 & -0.12 & 0.16 \\
\hline & Leaf dry weight & -0.05 & -0.10 & 0.05 & 0.02 & 0.10 & -0.23 & 0.10 & 0.08 & 0.10 & 0.07 & 0.08 & 0.01 & 0.15 & 0.03 & -0.14 & 0.30 & -0.15 \\
\hline & Leaf wet weight & -0.02 & -0.14 & 0.05 & 0.07 & 0.15 & -0.20 & 0.17 & 0.15 & 0.16 & 0.13 & 0.15 & -0.02 & 0.20 & 0.04 & -0.10 & 0.23 & -0.12 \\
\hline & Letiole length & -0.03 & -0.02 & 0.10 & 0.02 & 0.01 & -0.20 & -0.05 & -0.05 & -0.03 & -0.06 & -0.04 & 0.07 & 0.01 & 0.01 & -0.19 & 0.40 & -0.26 \\
\hline & Petiole diameter (max) & -0.02 & -0.12 & 0.03 & 0.02 & 0.13 & -0.19 & 0.12 & 0.10 & 0.11 & 0.11 & 0.10 & -0.03 & 0.15 & 0.05 & -0.08 & 0.26 & -0.12 \\
\hline & Petiole diameter (min) & -0.01 & -0.13 & 0.04 & 0.03 & 0.15 & -0.17 & 0.14 & 0.13 & 0.13 & 0.12 & 0.12 & -0.02 & 0.16 & 0.05 & -0.05 & 0.20 & -0.10 \\
\hline & Specific leaf area & 0.00 & -0.03 & -0.09 & 0.02 & -0.04 & 0.09 & 0.01 & 0.01 & 0.02 & -0.01 & 0.02 & 0.03 & -0.02 & 0.02 & -0.02 & -0.10 & -0.01 \\
\hline & SPAD & -0.05 & -0.08 & 0.20 & -0.03 & 0.01 & -0.15 & -0.05 & -0.05 & -0.04 & -0.04 & -0.04 & 0.02 & -0.01 & 0.08 & 0.00 & 0.20 & -0.05 \\
\hline & Stomatal density & -0.02 & 0.35 & -0.09 & -0.09 & -0.10 & 0.17 & -0.16 & -0.16 & -0.18 & -0.11 & -0.18 & -0.03 & -0.18 & -0.09 & 0.06 & -0.01 & -0.04 \\
\hline & Lignin content & 0.00 & 0.00 & -0.02 & -0.01 & -0.03 & -0.05 & -0.05 & -0.04 & -0.05 & -0.04 & -0.05 & 0.00 & -0.02 & 0.01 & -0.01 & 0.03 & -0.07 \\
\hline & S/G ratio & -0.07 & -0.03 & 0.10 & -0.09 & -0.07 & -0.08 & -0.07 & -0.05 & -0.03 & -0.09 & -0.03 & 0.08 & -0.04 & 0.03 & -0.11 & 0.18 & -0.17 \\
\hline & Early wood vessel count & 0.07 & -0.03 & -0.03 & 0.03 & 0.04 & 0.06 & 0.04 & 0.03 & 0.01 & 0.05 & 0.02 & -0.03 & 0.05 & -0.01 & 0.09 & -0.13 & 0.12 \\
\hline & Early wood vessel area & 0.08 & -0.09 & -0.08 & 0.09 & 0.09 & 0.09 & 0.13 & 0.13 & 0.11 & 0.13 & 0.11 & -0.06 & 0.13 & -0.09 & 0.07 & -0.16 & 0.15 \\
\hline & Early wood vessel size & -0.02 & -0.06 & -0.06 & 0.05 & 0.04 & 0.04 & 0.05 & 0.07 & 0.07 & 0.05 & 0.06 & -0.01 & 0.05 & -0.01 & -0.04 & -0.03 & 0.03 \\
\hline & Intermediate wood vessel count & 0.06 & 0.07 & 0.02 & -0.01 & -0.01 & 0.00 & 0.02 & 0.00 & -0.02 & 0.01 & -0.01 & -0.03 & 0.03 & 0.01 & 0.07 & -0.05 & 0.06 \\
\hline & Intermediate wood vessel area & 0.06 & -0.03 & 0.01 & 0.02 & 0.03 & -0.02 & 0.09 & 0.09 & 0.06 & 0.09 & 0.07 & -0.05 & 0.09 & -0.02 & 0.10 & -0.10 & 0.12 \\
\hline & Intermediate wood vessel size & -0.06 & -0.10 & -0.02 & 0.03 & 0.06 & -0.01 & 0.09 & 0.10 & 0.10 & 0.09 & 0.10 & -0.01 & 0.05 & -0.02 & 0.03 & -0.11 & 0.05 \\
\hline & Late wood vessel count & 0.05 & -0.03 & -0.07 & 0.03 & -0.01 & 0.04 & 0.02 & 0.01 & 0.00 & 0.02 & 0.01 & -0.01 & 0.04 & 0.01 & 0.06 & 0.01 & 0.05 \\
\hline & Late wood vessel area & 0.05 & -0.05 & 0.00 & 0.14 & 0.10 & -0.01 & 0.13 & 0.13 & 0.11 & 0.13 & 0.11 & -0.06 & 0.14 & -0.05 & 0.15 & -0.16 & 0.19 \\
\hline & Late wood vessel size & -0.05 & -0.02 & 0.06 & 0.06 & 0.04 & -0.03 & 0.06 & 0.09 & 0.07 & 0.06 & 0.06 & -0.04 & 0.04 & -0.02 & 0.09 & -0.16 & 0.11 \\
\hline
\end{tabular}


Table 4.5 Pearson correlation (r) of P. trichocarpa wood chemistry, anatomical and morphological traits in Clatskanie, OR with morphological and physiological traits in Corvallis, OR. Values greater than 0.15 or less than -0.15 are significant based on the Bonferroni correction criteria at $5 \%$ significance level.

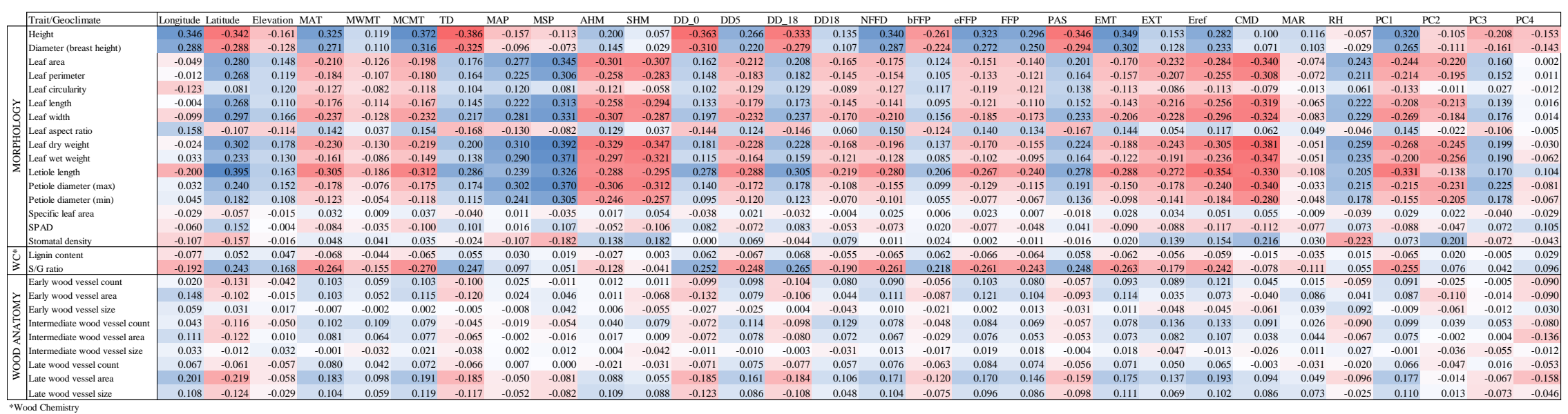


Table 4.6 Genes identified from $P$. trichocarpa single trait GWAS.

\begin{tabular}{|c|c|c|c|}
\hline Gene model $^{\mathrm{a}}$ & Trait & $P$-value ${ }^{\mathrm{b}}$ & Functional annotation \\
\hline Potri.014G117300 & $\begin{array}{l}\text { Diameter (breast } \\
\text { height) }\end{array}$ & $1.95 \mathrm{E}-08$ & Gibberellin 2-beta-dioxygenase 2 \\
\hline Potri.014G117400 & $\begin{array}{l}\text { Diameter (breast } \\
\text { height) }\end{array}$ & $1.88 \mathrm{E}-08$ & similar to MYB family transcription factor \\
\hline Potri.018G141400 & $\begin{array}{l}\text { Diameter (breast } \\
\text { height) }\end{array}$ & $5.67 \mathrm{E}-08$ & $\mathrm{ABC}$ transporter \\
\hline Potri.005G247400 & Leaf circularity & $9.20 \mathrm{E}-08$ & similar to prolyl oligopeptidase \\
\hline Potri.005G247600 & Leaf circularity & 4.63E-08 & $\begin{array}{l}\text { similar to maoC-like dehydratase domain- } \\
\text { containing protein }\end{array}$ \\
\hline Potri.005G247700 & Leaf circularity & $1.37 \mathrm{E}-08$ & multi-copper oxidase type 1 family protein \\
\hline Potri.005G247900 & Leaf circularity & $9.01 \mathrm{E}-08$ & ubiquitin family protein \\
\hline Potri.019G042600 & $\begin{array}{l}\text { Leaf dry weight, leaf } \\
\text { wet weight* }\end{array}$ & 1.49E-08 & $\begin{array}{l}\text { MITOCHONDRIAL OUTER } \\
\text { MEMBRANE PROTEIN } 25\end{array}$ \\
\hline Potri.019G042700 & $\begin{array}{l}\text { Leaf dry weight, leaf } \\
\text { wet weight }\end{array}$ & 9.73E-09 & $\begin{array}{l}\text { similar to expressed protein in Arabidopsis } \\
\text { thaliana (co-ortholog of At } 4 \mathrm{~g} 27620 \text {, } \\
\text { At } 4 \mathrm{~g} 27610 \text { ) }\end{array}$ \\
\hline Potri.015G117300 & Stomatal density & $6.49 \mathrm{E}-09$ & NA \\
\hline Potri.015G117400 & Stomatal density & $6.11 \mathrm{E}-08$ & PROTEIN ARGONAUTE 2-RELATED \\
\hline Potri.015G117500 & Stomatal density & 2.77E-09 & $\begin{array}{l}\text { Peptide-O-fucosyltransferase/GDP-L- } \\
\text { fucose:polypeptide fucosyltransferase }\end{array}$ \\
\hline Potri.015G117600 & Stomatal density & 8.31E-08 & $\begin{array}{l}\text { zinc finger (C3HC4-type RING finger) } \\
\text { family protein; similar to } \mathrm{C} 3 \mathrm{HC} 4 \text { type } \\
\text { (RING finger) }\end{array}$ \\
\hline Potri.002G238700 & Leaf weight weight & 5.74E-08 & $\begin{array}{l}\text { similar to hypothetical protein (co-ortholog } \\
\text { (1of2) of At5g48890) }\end{array}$ \\
\hline Potri.001G311900 & Leaf aspect ratio & 2.29E-08 & $\begin{array}{l}\text { Cyanohydrin beta- } \\
\text { glucosyltransferase/Uridine } \\
\text { diphosphoglucose:aldehyde cyanohydrin } \\
\text { beta-glucosyltransferase }\end{array}$ \\
\hline Potri.006G1 19400 & $\mathrm{~S} / \mathrm{G}$ ratio & $9.15 \mathrm{E}-08$ & $\begin{array}{l}\text { PLATZ TRANSCRIPTION FACTOR } \\
\text { FAMILY PROTEIN }\end{array}$ \\
\hline Potri.004G141500 & $\begin{array}{l}\text { Early wood vessel } \\
\text { count }\end{array}$ & $8.11 \mathrm{E}-08$ & $\begin{array}{l}\text { DNA repair and recombination protein } \\
\text { RAD54 and RAD54-like protein }\end{array}$ \\
\hline Potri.012G007600 & $\begin{array}{l}\text { Intermediate wood } \\
\text { vessel size }\end{array}$ & $9.58 \mathrm{E}-08$ & $\begin{array}{l}\text { CARBON CATABOLITE REPRESSOR } \\
\text { PROTEIN } 4\end{array}$ \\
\hline Potri.019G037900 & $\begin{array}{l}\text { Intermediate wood } \\
\text { vessel size }\end{array}$ & $8.44 \mathrm{E}-09$ & $\begin{array}{l}\text { Staufen and related double-stranded-RNA- } \\
\text { binding proteins }\end{array}$ \\
\hline Potri.006G205700 & Late wood vessel size & $3.17 \mathrm{E}-08$ & $\begin{array}{l}\text { ubiquitin-conjugating enzyme family } \\
\text { protein; similar to DNA-binding protein } \\
\text { CROC-1B (Homo sapiens) }\end{array}$ \\
\hline
\end{tabular}

${ }^{\mathrm{a}}$ Gene models are annotated using v3 of the $P$. trichocarpa genome

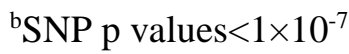

*smallest $P$-value reported (leaf dry weight) 
Table 4.7 Genes identified from P. trichocarpa multitrait GWAS.

\begin{tabular}{|c|c|c|c|}
\hline Gene model ${ }^{\mathrm{a}}$ & Trait & $P$-value & Functional annotation \\
\hline Potri.001G085400 & $\begin{array}{l}\text { Early wood vessel area, early wood } \\
\text { vessel count }\end{array}$ & 3.07E-08 & $\begin{array}{l}\text { similar to Yippee-like protein } \\
\text { (co-ortholog of At4g27740) }\end{array}$ \\
\hline Potri.005G038400 & $\begin{array}{l}\text { Early wood vessel area, early wood } \\
\text { vessel count }\end{array}$ & 7.70E-08 & PPR repeat family (PPR_2) \\
\hline Potri.001G140500 & $\begin{array}{l}\text { Early wood vessel area, early wood } \\
\text { vessel count, intermediate wood } \\
\text { vessel area, intermediate wood } \\
\text { vessel count, lignin content, late } \\
\text { wood vessel area, late wood vessel } \\
\text { count, S/G ratio }\end{array}$ & 2.29E-08 & $\begin{array}{l}\text { CARBOXYLATE CLAMP- } \\
\text { TETRATRICOPEPTIDE } \\
\text { REPEAT PROTEIN }\end{array}$ \\
\hline Potri.002G108000 & $\begin{array}{l}\text { Early wood vessel area, early wood } \\
\text { vessel count, intermediate wood } \\
\text { vessel area, intermediate wood } \\
\text { vessel count, lignin content, late } \\
\text { wood vessel area, late wood vessel } \\
\text { count, } \text { S/G ratio }\end{array}$ & $6.31 \mathrm{E}-08$ & $\begin{array}{l}\text { Auxin responsive-protein } \\
\text { IAA8 }\end{array}$ \\
\hline Potri.006G275900 & $\begin{array}{l}\text { Early wood vessel area, early wood } \\
\text { vessel count, intermediate wood } \\
\text { vessel area, intermediate wood } \\
\text { vessel count, lignin content, late } \\
\text { wood vessel area, late wood vessel } \\
\text { count, S/G ratio }\end{array}$ & 4.16E-08 & $\begin{array}{l}\text { MYB-LIKE DNA-BINDING } \\
\text { PROTEIN }\end{array}$ \\
\hline Potri.008G160600 & $\begin{array}{l}\text { Early wood vessel area, early wood } \\
\text { vessel count, intermediate wood } \\
\text { vessel area, intermediate wood } \\
\text { vessel count, lignin content, late } \\
\text { wood vessel area, late wood vessel } \\
\text { count, } S / G \text { ratio }\end{array}$ & $8.15 \mathrm{E}-08$ & $\begin{array}{l}\text { Non-specific serine/threonine } \\
\text { protein kinase }\end{array}$ \\
\hline Potri.017G090200 & $\begin{array}{l}\text { Early wood vessel area, early wood } \\
\text { vessel count, intermediate wood } \\
\text { vessel area, intermediate wood } \\
\text { vessel count, lignin content, late } \\
\text { wood vessel area, late wood vessel } \\
\text { count, S/G ratio }\end{array}$ & 7.32E-09 & $\begin{array}{l}\text { CALMODULIN-BINDING } \\
\text { PROTEIN-LIKE PROTEIN }\end{array}$ \\
\hline Potri.017G110300 & $\begin{array}{l}\text { Early wood vessel area, early wood } \\
\text { vessel count, intermediate wood } \\
\text { vessel area, intermediate wood } \\
\text { vessel count, lignin content, late } \\
\text { wood vessel area, late wood vessel } \\
\text { count, S/G ratio }\end{array}$ & 7.63E-08 & NA \\
\hline Potri.006G275800 & $\begin{array}{l}\text { Early wood vessel area, early wood } \\
\text { vessel count, intermediate wood } \\
\text { vessel area, intermediate wood } \\
\text { vessel count, lignin content, late } \\
\text { wood vessel area, late wood vessel }\end{array}$ & $2.37 \mathrm{E}-10$ & $\begin{array}{l}\text { PTHR23155//PTHR23155:SF } \\
609 \text { - LEUCINE-RICH } \\
\text { REPEAT-CONTAINING } \\
\text { PROTEIN // SUBFAMILY } \\
\text { NOT NAMED }\end{array}$ \\
\hline
\end{tabular}




\begin{tabular}{|c|c|c|c|}
\hline 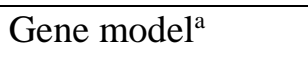 & Trait & $P$-value & Functional annotation \\
\hline Potri.014G171300 & $\begin{array}{l}\text { count, S/G ratio* and Intermediate } \\
\text { wood vessel area, intermediate } \\
\text { wood vessel count } \\
\text { Early wood vessel area, early wood } \\
\text { vessel count, intermediate wood } \\
\text { vessel area, intermediate wood } \\
\text { vessel count, late wood vessel area, } \\
\text { late wood vessel count }\end{array}$ & $9.71 \mathrm{E}-08$ & $\begin{array}{l}\text { similar to heavy-metal- } \\
\text { associated domain-containing } \\
\text { protein; similar to strong } \\
\text { similarity to farnesylated } \\
\text { proteins ATFP4 }\end{array}$ \\
\hline Potri.004G002600 & $\begin{array}{l}\text { Early wood vessel area, leaf area, } \\
\text { stomotal density and early wood } \\
\text { vessel area, leaf area, stomatal } \\
\text { density }\end{array}$ & $9.57 \mathrm{E}-09$ & $\begin{array}{l}\text { (1 of 2) PTHR } 31803: \text { SF } 10 \text { - } \\
\text { UBIQUINOL OXIDASE } 4 \text {, } \\
\text { CHLOROPLASTIC/CHRO } \\
\text { MOPLASTIC }\end{array}$ \\
\hline Potri.016G013400 & $\begin{array}{l}\text { Early wood vessel area, leaf area, } \\
\text { stomotal density }\end{array}$ & $3.20 \mathrm{E}-08$ & $\begin{array}{l}\text { (1 of 3) K15223 - upstream } \\
\text { activation factor subunit } \\
\text { UAF30 (UAF30, SPP27) }\end{array}$ \\
\hline Potri.015G113800 & $\begin{array}{l}\text { Early wood vessel area, leaf area, } \\
\text { stomotal density*** and lignin } \\
\text { content, specific leaf area, stomatal } \\
\text { density, early wood vessel area }\end{array}$ & $2.22 \mathrm{E}-08$ & $\begin{array}{l}\text { Protein of unknown function, } \\
\text { DUF594 (DUF594) // } \\
\text { Domain of unknown function } \\
\text { (DUF4220) (DUF4220) }\end{array}$ \\
\hline Potri.011G022200 & $\begin{array}{l}\text { Early wood vessel count, } \\
\text { intermediate wood vessel count, late } \\
\text { wood vessel count }\end{array}$ & $2.78 \mathrm{E}-08$ & $\begin{array}{l}\text { (1 of 3) PTHR33076:SF6 - } \\
\text { NON-SPECIFIC LIPID- } \\
\text { TRANSFER PROTEIN } 15\end{array}$ \\
\hline Potri.009G149900 & $\begin{array}{l}\text { Early wood vessel size, intermediate } \\
\text { wood vessel size, lignin content, } \\
\text { S/G ratio, late wood vessel size }\end{array}$ & $8.52 \mathrm{E}-08$ & $\begin{array}{l}\text { amino acid transporter family } \\
\text { protein; similar to low } \\
\text { similarity to amino acid } \\
\text { permease (Oryza sativa) } \\
\text { GI:7415521 }\end{array}$ \\
\hline Potri.T031400 & $\begin{array}{l}\text { Early wood vessel size, intermediate } \\
\text { wood vessel size, lignin content, } \\
\text { S/G ratio, late wood vessel size }\end{array}$ & $9.42 \mathrm{E}-08$ & NA \\
\hline Potri.001G058100 & $\begin{array}{l}\text { Intermediate wood vessel area, } \\
\text { intermediate wood vessel count }\end{array}$ & 4.15E-08 & $\begin{array}{l}\text { similar to expressed protein in } \\
\text { Arabidopsis thaliana (co- } \\
\text { ortholog of At1g27290) }\end{array}$ \\
\hline Potri.019G037900 & $\begin{array}{l}\text { Intermediate wood vessel size, } \\
\text { lignin content, } S / G \text { ratio }\end{array}$ & $1.77 \mathrm{E}-09$ & $\begin{array}{l}\text { Staufen and related double- } \\
\text { stranded-RNA-binding } \\
\text { proteins }\end{array}$ \\
\hline Potri.001G467800 & $\begin{array}{l}\text { Intermediate wood vessel size, } \\
\text { lignin content, S/G ratio** and } \\
\text { Lignin content, late wood vessel } \\
\text { size, S/G ratio }\end{array}$ & $3.69 \mathrm{E}-08$ & $\begin{array}{l}\text { similar to uracil } \\
\text { phosphoribosyltransferase }\end{array}$ \\
\hline Potri.004G056300 & $\begin{array}{l}\text { Leaf area, leaf dry weight, leaf } \\
\text { length, leaf width }\end{array}$ & $6.88 \mathrm{E}-12$ & Fruit bromelain \\
\hline Potri.007G061600 & $\begin{array}{l}\text { Leaf area, leaf dry weight, leaf } \\
\text { length, leaf width }\end{array}$ & $1.03 \mathrm{E}-09$ & $\begin{array}{l}\text { vacuolar protein sorting- } \\
\text { associated protein } 35 \\
\text { (VPS35) }\end{array}$ \\
\hline Potri.007G099700 & Leaf area, leaf dry weight, leaf & 8.07E-08 & Leucine rich repeat (LRR_8) \\
\hline
\end{tabular}




\begin{tabular}{|c|c|c|c|}
\hline 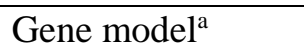 & Trait & $P$-value ${ }^{\mathrm{b}}$ & Functional annotation \\
\hline Potri.010G031900 & $\begin{array}{l}\text { Leaf area, leaf dry weight, leaf } \\
\text { length, leaf width }\end{array}$ & $5.23 \mathrm{E}-08$ & $\begin{array}{l}\text { similar to RUB-activating } \\
\text { enzyme (Ubiquitin activating } \\
\text { enzyme E1 like protein) }\end{array}$ \\
\hline Potri.010G032600 & $\begin{array}{l}\text { Leaf area, leaf dry weight, leaf } \\
\text { length, leaf width }\end{array}$ & $3.62 \mathrm{E}-13$ & $\begin{array}{l}\text { small subunit ribosomal } \\
\text { protein S11 (RP-S11, } \\
\text { MRPS11, rpsK) }\end{array}$ \\
\hline Potri.012G028700 & $\begin{array}{l}\text { Leaf area, leaf dry weight, leaf } \\
\text { length, leaf width }\end{array}$ & $5.90 \mathrm{E}-09$ & $\begin{array}{l}\text { Ras suppressor protein } \\
\text { (contains leucine-rich repeats) }\end{array}$ \\
\hline Potri.019G067300 & $\begin{array}{l}\text { Leaf area, leaf dry weight, leaf } \\
\text { length, leaf width }\end{array}$ & $1.90 \mathrm{E}-08$ & $\begin{array}{l}\text { (1 of 1) PTHR11214:SF125 - } \\
\text { BETA-1,3- } \\
\text { GALACTOSYLTRANSFER } \\
\text { ASE 8-RELATED }\end{array}$ \\
\hline Potri.005G054400 & $\begin{array}{l}\text { Lignin content, late wood vessel } \\
\text { size, } S / G \text { ratio }\end{array}$ & $3.10 \mathrm{E}-08$ & $\begin{array}{l}\text { RING FINGER DOMAIN- } \\
\text { CONTAINING, } \\
\text { POLYCOMB GROUP } \\
\text { COMPONENT }\end{array}$ \\
\hline Potri.006G242100 & $\begin{array}{l}\text { Lignin content, late wood vessel } \\
\text { size, } S / G \text { ratio }\end{array}$ & $5.48 \mathrm{E}-08$ & $\begin{array}{l}\text { similar to expressed protein in } \\
\text { Arabidopsis thaliana } \\
\text { (ortholog of At2g25737) }\end{array}$ \\
\hline Potri.006G242200 & $\begin{array}{l}\text { Lignin content, late wood vessel } \\
\text { size, S/G ratio }\end{array}$ & 8.37E-08 & PPR repeat family (PPR_2) \\
\hline Potri.009G137800 & Lignin content, $\mathrm{S} / \mathrm{G}$ ratio & $1.42 \mathrm{E}-08$ & $\begin{array}{l}\text { similar to vacuolar ATP } \\
\text { synthase subunit B }\end{array}$ \\
\hline Potri.012G047300 & Lignin content, $\mathrm{S} / \mathrm{G}$ ratio & 4.57E-08 & METHYLTRANSFERASE \\
\hline Potri.004G183900 & $\begin{array}{l}\text { Late wood vessel area, late wood } \\
\text { vessel count }\end{array}$ & 5.91E-09 & $\begin{array}{l}\text { SERINE/THREONINE- } \\
\text { PROTEIN KINASE }\end{array}$ \\
\hline
\end{tabular}

${ }^{\mathrm{a}}$ Gene models are annotated using v3 of the $P$. trichocarpa genome

${ }^{\text {b}} \mathrm{SNP} \mathrm{p}$ values $<1 \times 10^{-7}$

*Early wood vessel area, early wood vessel count, intermediate wood vessel area, intermediate wood vessel count, lignin content, late wood vessel area, late wood vessel count, S/G ratio with lowest $P$ value

**Intermediate wood vessel size, lignin content, $\mathrm{S} / \mathrm{G}$ ratio with lowest $P$-value ***Early wood vessel area, leaf area, stomatal density with lowest $P$-value 


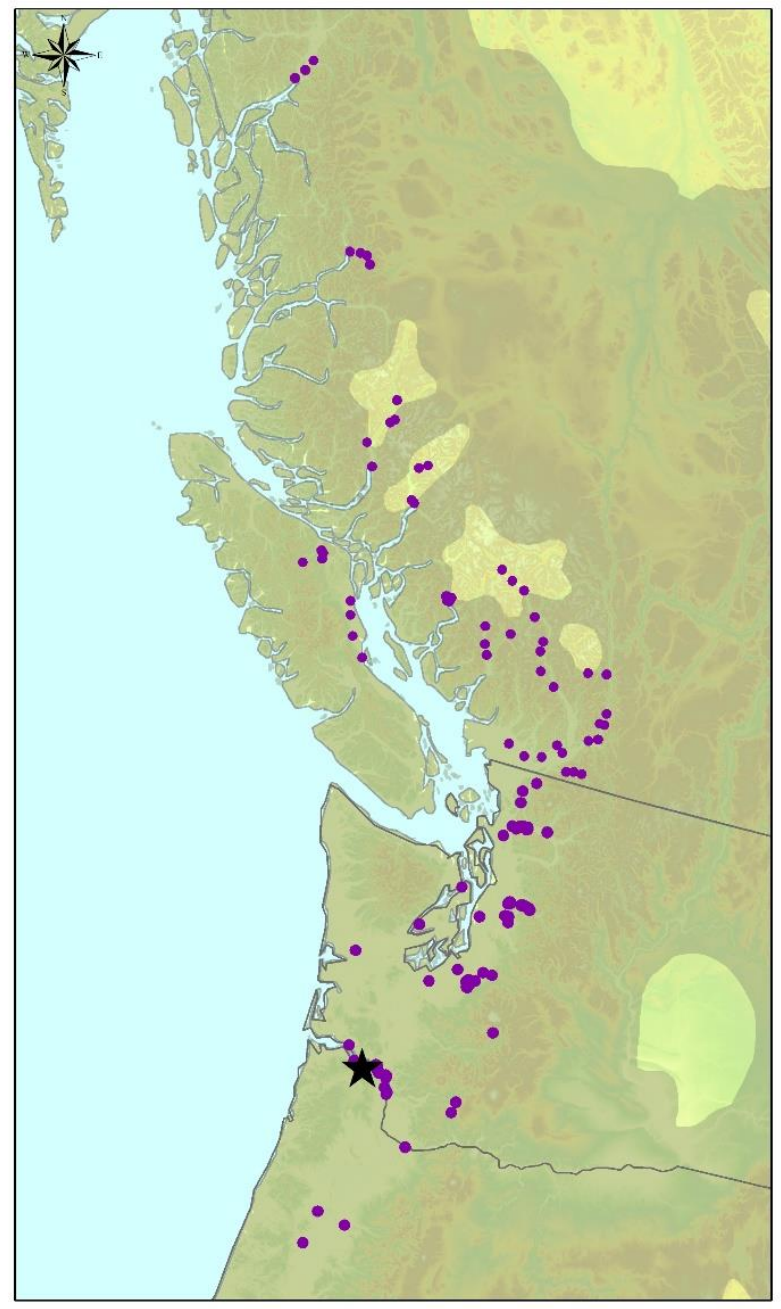

Figure 4.1 Source locations of 869 P. trichocarpa genotypes sampled in this study (purple dots). The trees were grown in a common garden in Clatskanie, Oregon, USA (black star).

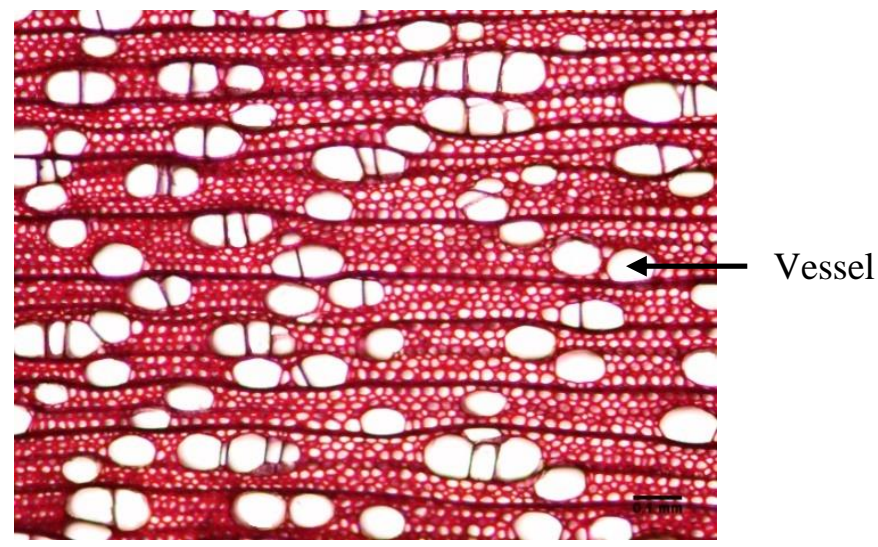

Figure 4.2 Representative $P$. trichocarpa stem cross section at 100x magnification. 
(a)

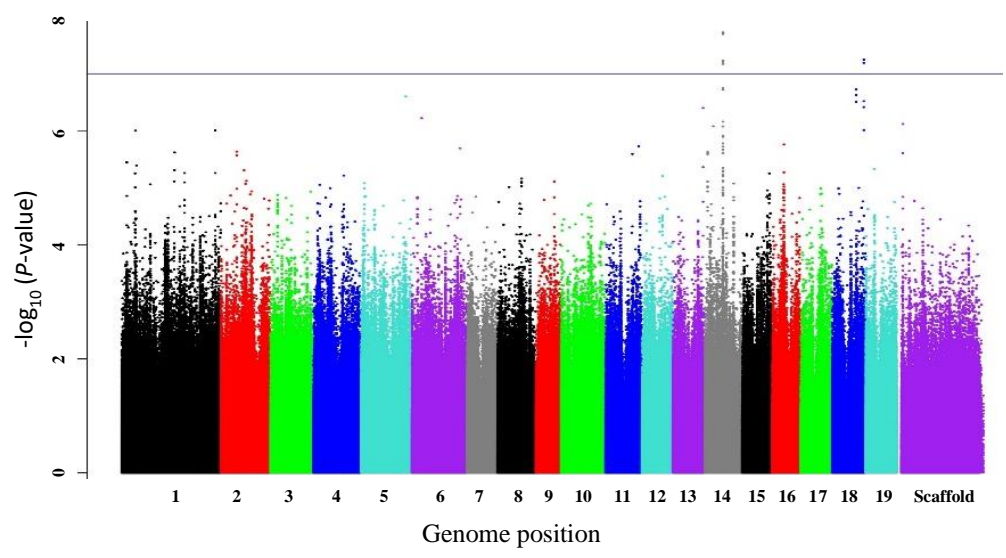

(c)

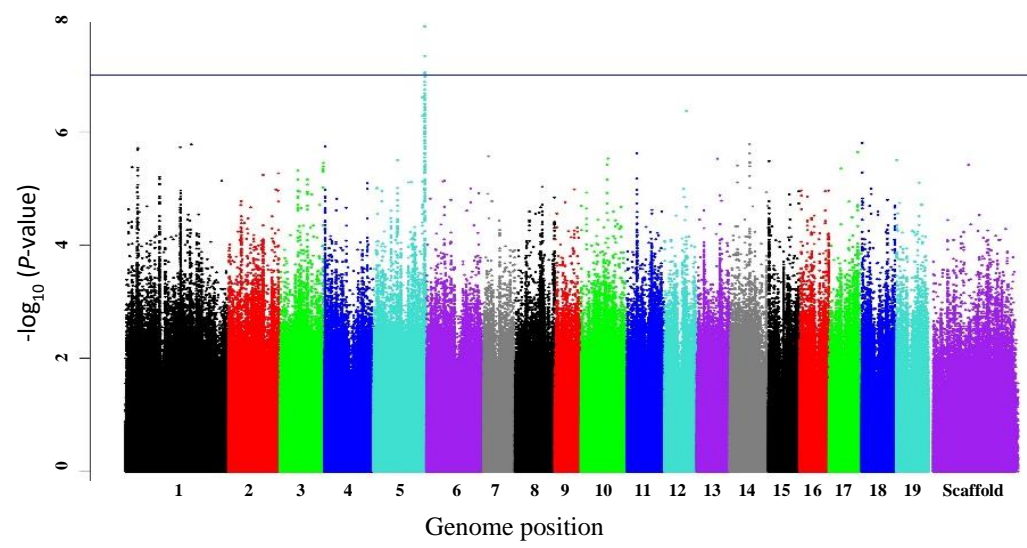

(e)

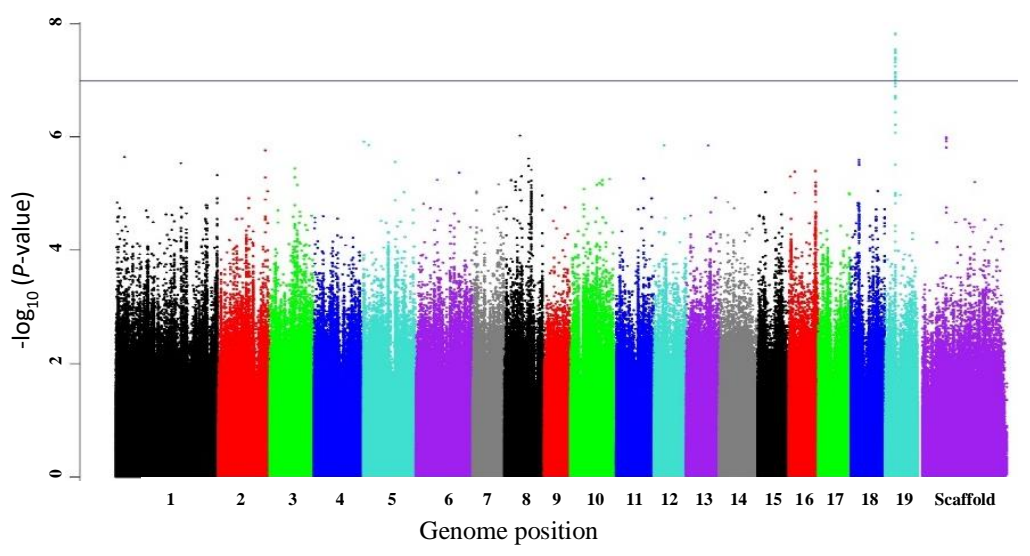

(b)

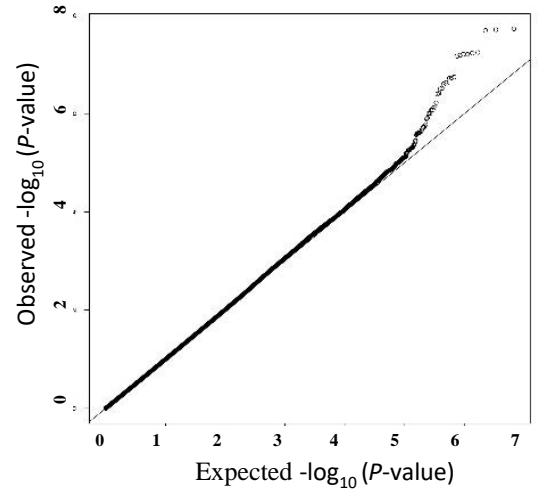

(d)

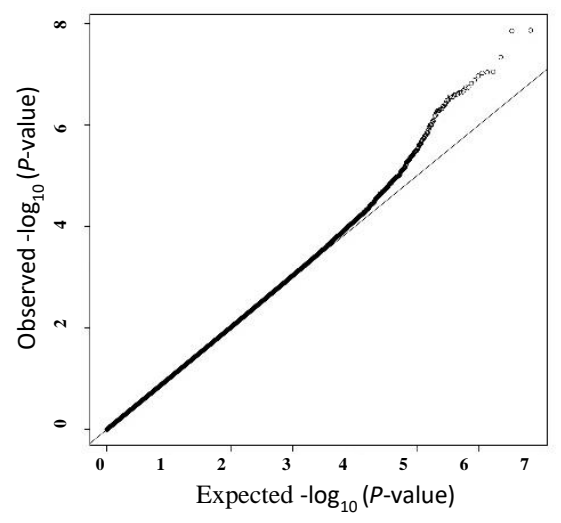

(f)

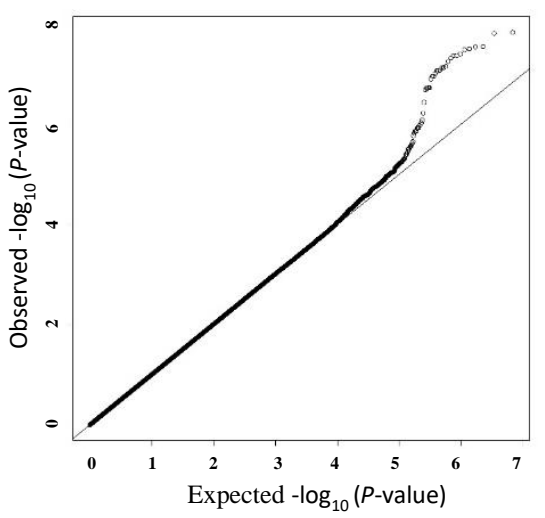


(g)

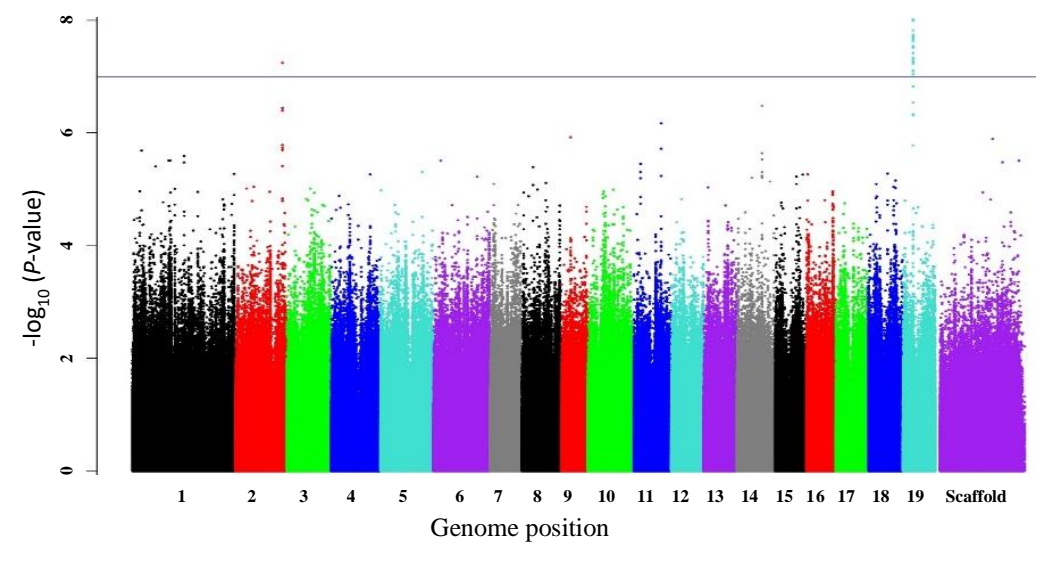

(i)

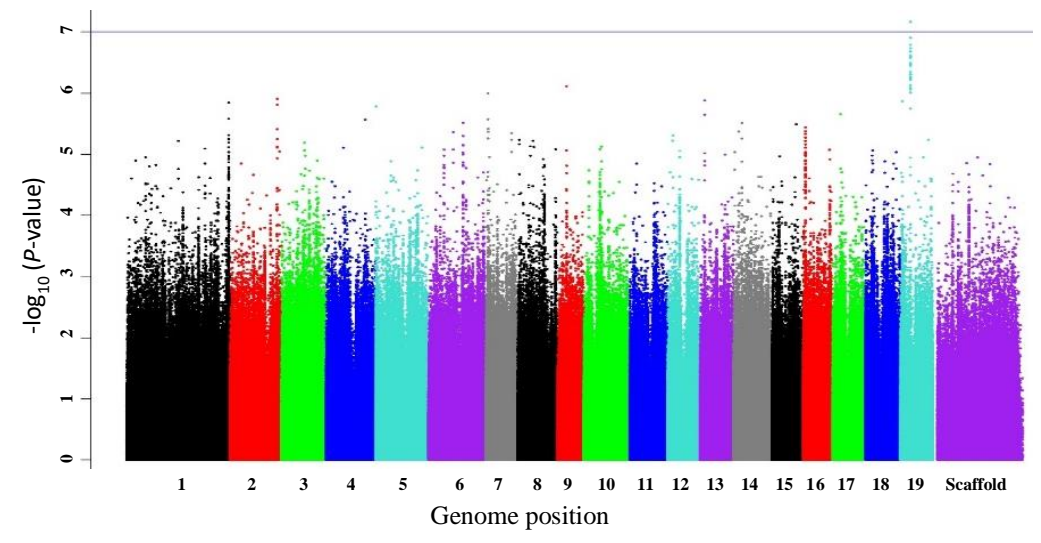

(k)

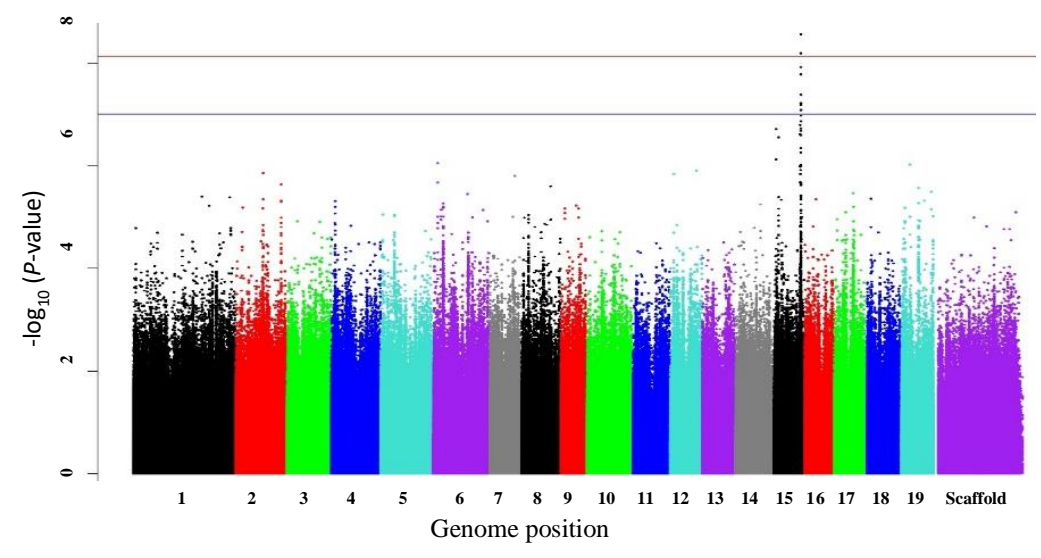

(h)

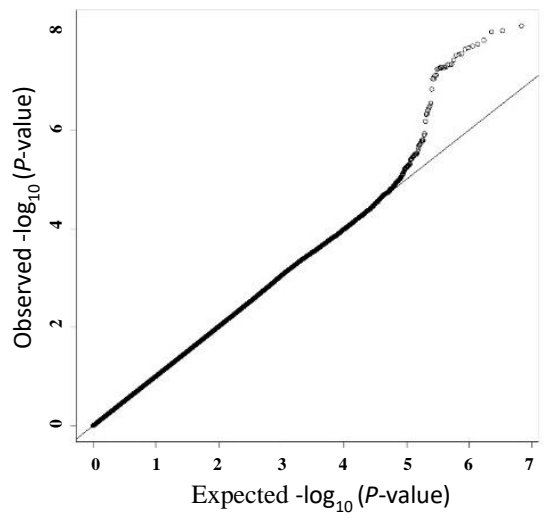

(j)

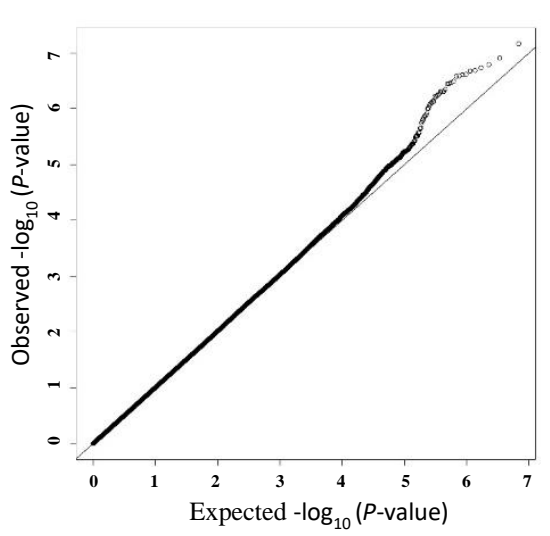

(1)

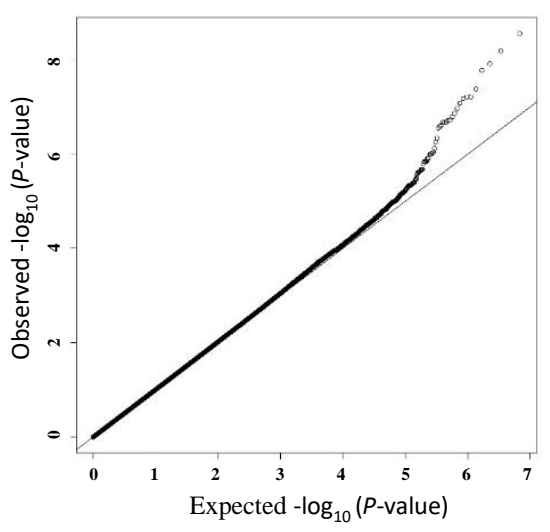



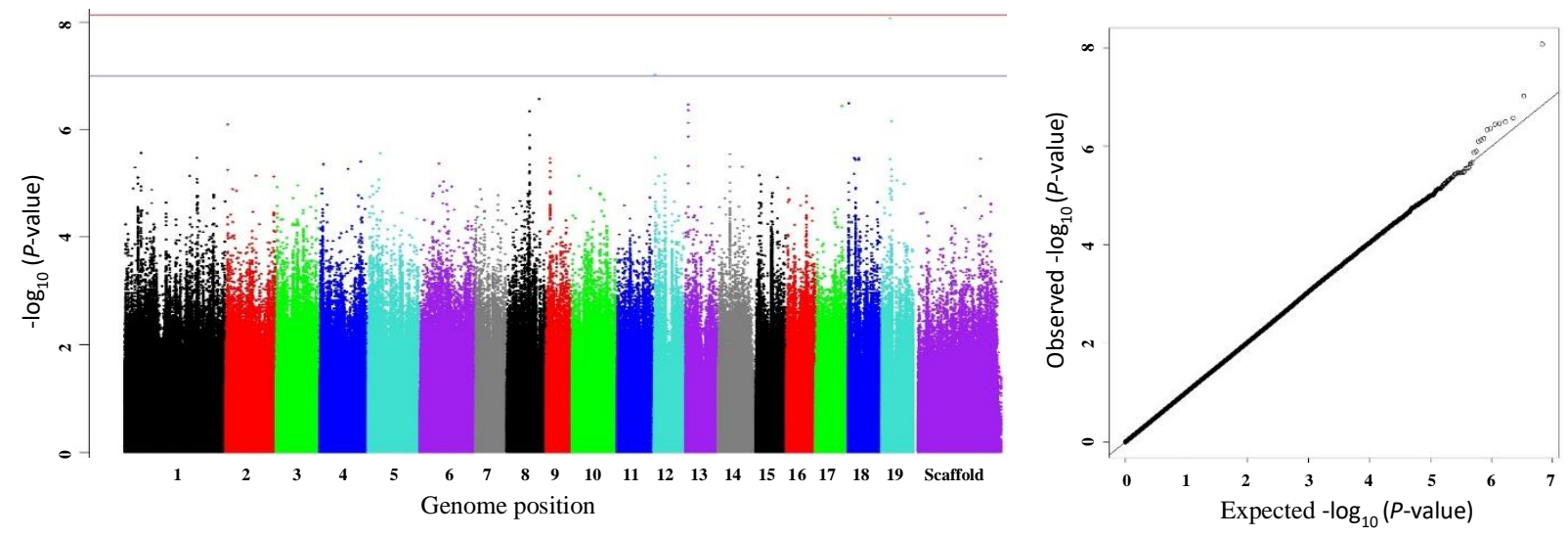

Figure 4.3 Single trait GWAS - Manhattan (left) and QQ plots (right). Numbers 1 to 19 represent chromosomes; scaffolds are the reads that did not align to any of the 19 chromosomes. (a) and (b) DBH; (c) and (d) Leaf circularity; (e) and (f) Leaf dry weight; (g) and (h) Leaf wet weight; (i) and (j) Petiole maximum diameter; (k) and (l) Abaxial stomatal density; (m) and (n) Intermediate wood vessel size. Red horizontal line indicates Bonferroni correction threshold $\left(P=7.417 \times 10^{-9}\right)$ and blue horizontal line indicates suggestive association threshold $\left(P=1 \times 10^{-7}\right)$. 
(a)

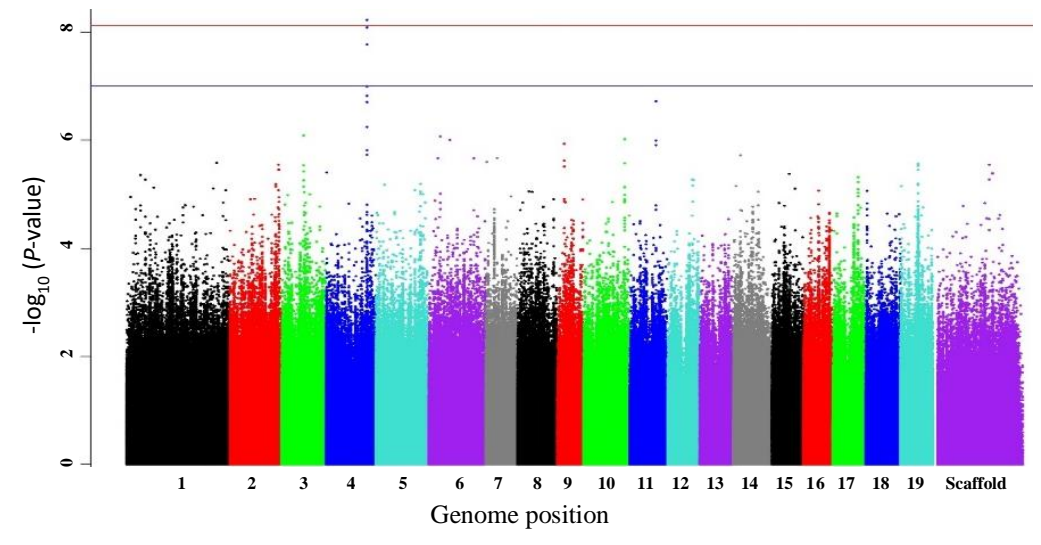

(c)

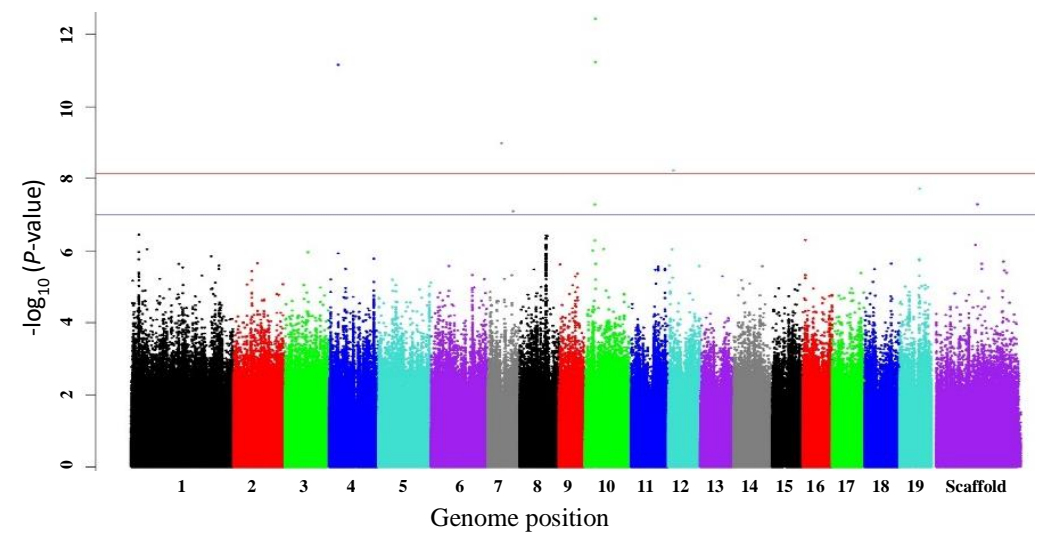

(b)

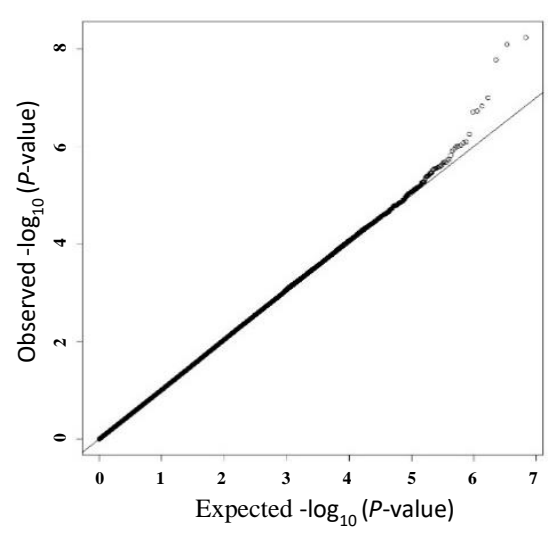

(d)

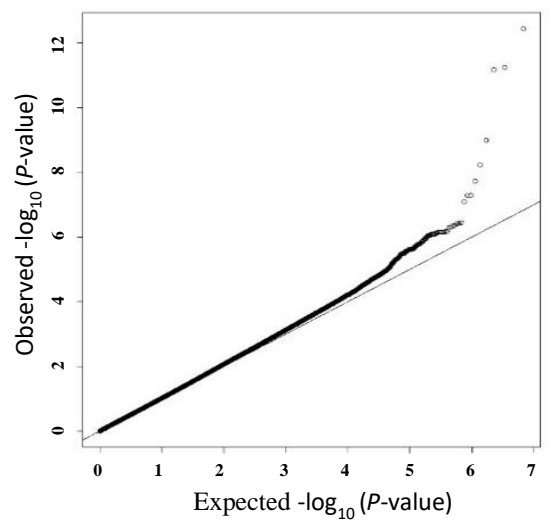

Figure 4.4 Multitrait GWAS - Manhattan (left) and QQ plots (right). Numbers 1 to 19 represent chromosomes; scaffolds are the reads that did not align to any of the 19 chromosomes. (a) and (b) Late wood vessel area and Late wood vessel count; (c) and (d) Leaf area, leaf dry weight, leaf length and leaf wet weight. Red horizontal line indicates Bonferroni correction threshold $\left(P=7.417 \times 10^{-9}\right)$ and blue horizontal line indicates suggestive association threshold $\left(P=1 \times 10^{-7}\right)$. 

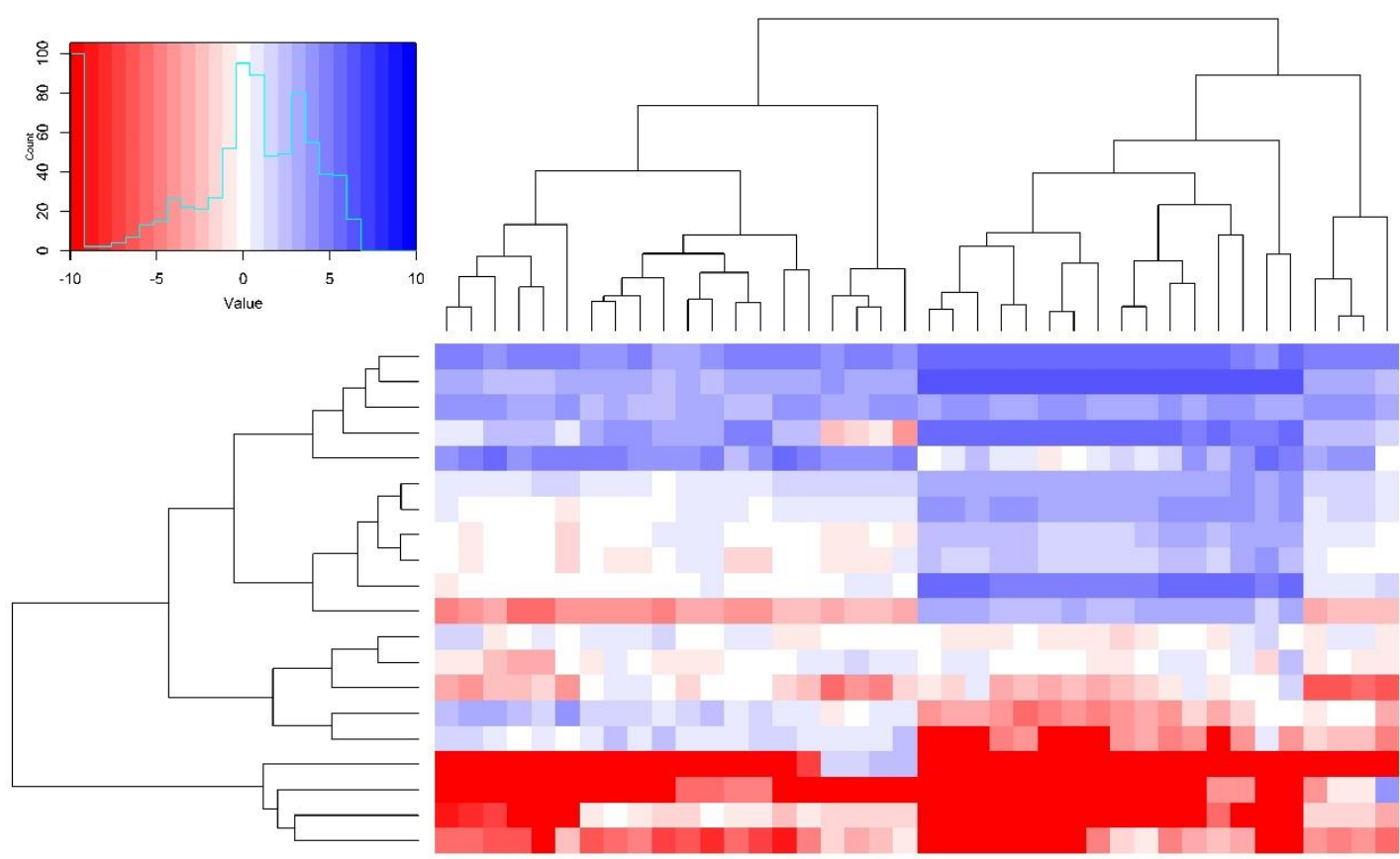

Potri.015G117600 Potri.006G205700

Potri.005G247400

Potri.019G042600

Potri.005G247700

Potri.005G247600

Potri.014G117400

Potri.019G042700

Potri.004G141500

Potri.005G247900

Potri.019G037900

Potri.015G117400

Potri.015G117500

Potri.015G117300

Potri.012G007600

Potri.006G119400

Potri.001G311900

Potri.002G238700

Potri.018G141400

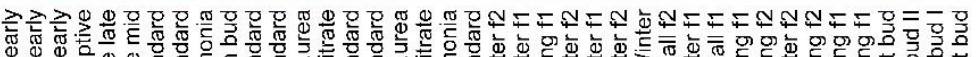

Potri.014G117300

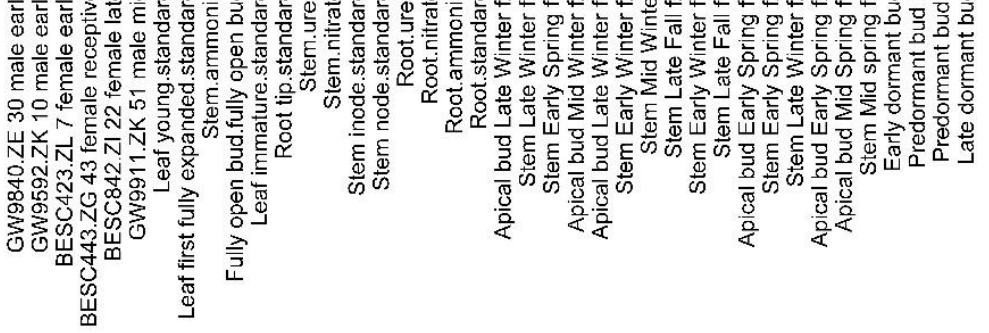

Figure 4.5 Expression level of gene models identified from single trait GWAS in different tissues in $P$.

trichocarpa. 

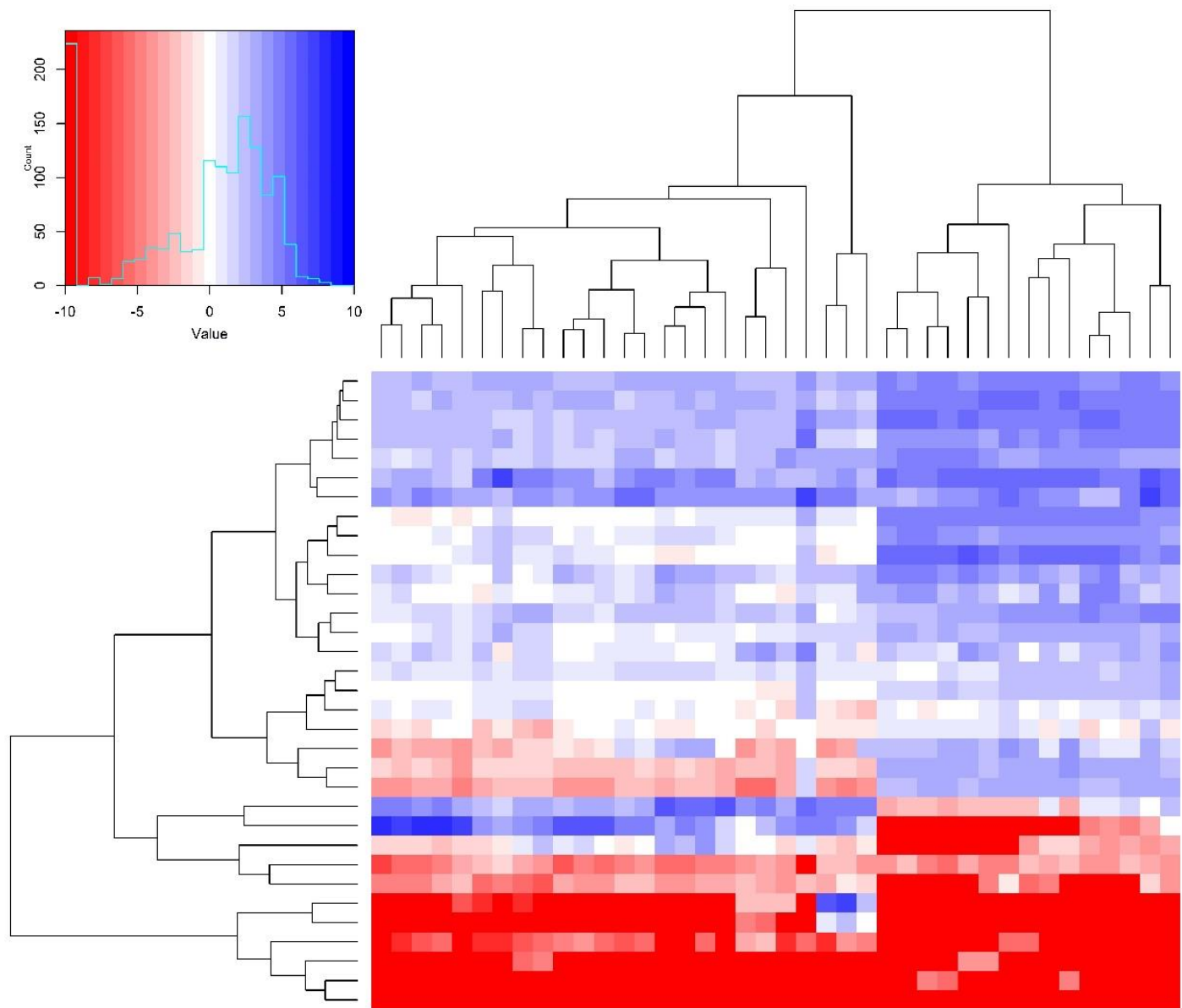

Potri.010G031900 Potri.008G160400 Potri.009G137800 Potri.016G013400 Potri.017G090200 Potri.001G140500 Potri.002G108000 Potri.001G467800 Potri.007G099700 Potri.004G002600 Potri.001G058100 Potri.009G149900 Potri.008G160600 Potri.006G242200 Potri.012G047300 Potri.001G085400 Potri.017G110300 Potri.005G038400 Potri.006G275800 Potri.004G183900 Potri.004G183900 Potri.005G054400 Potri.006 242100 Potri.006G242100 Potri.006G275900 Potri.014G171300 Potri.007G061600 Potri.015G113800 Potri.019G067300 Potri.011G022200 Potri.004G056300 Potri.012G028700 Potri.T031400 Potri.010G032600

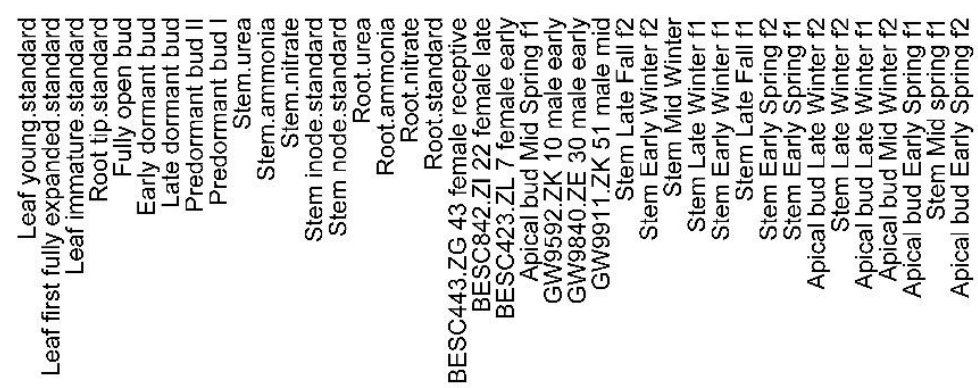

Figure 4.6 Expression level of gene models identified from multitrait GWAS in different tissues in $P$. trichocarpa. 


\subsection{Supplementary figures}

(a)

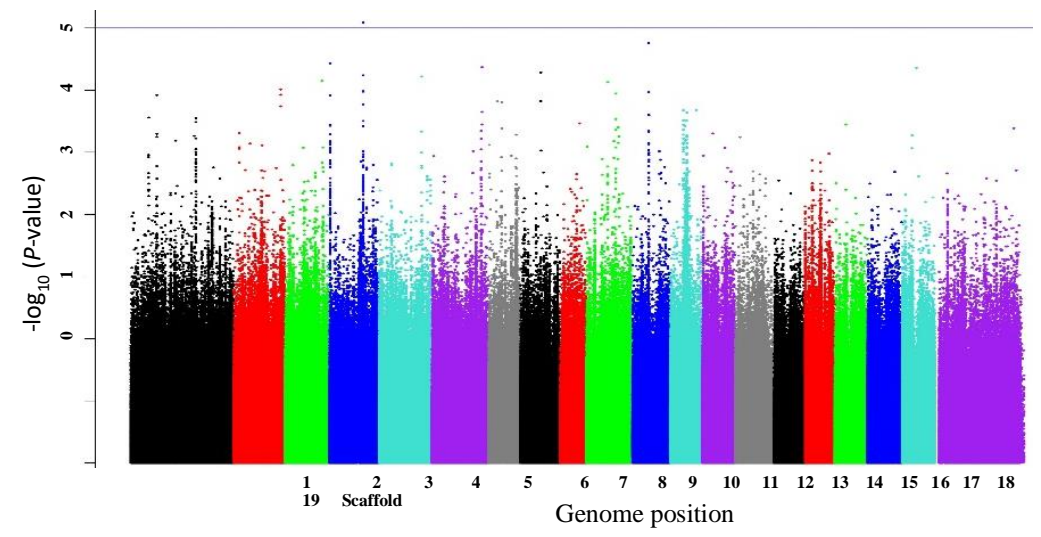

(c)

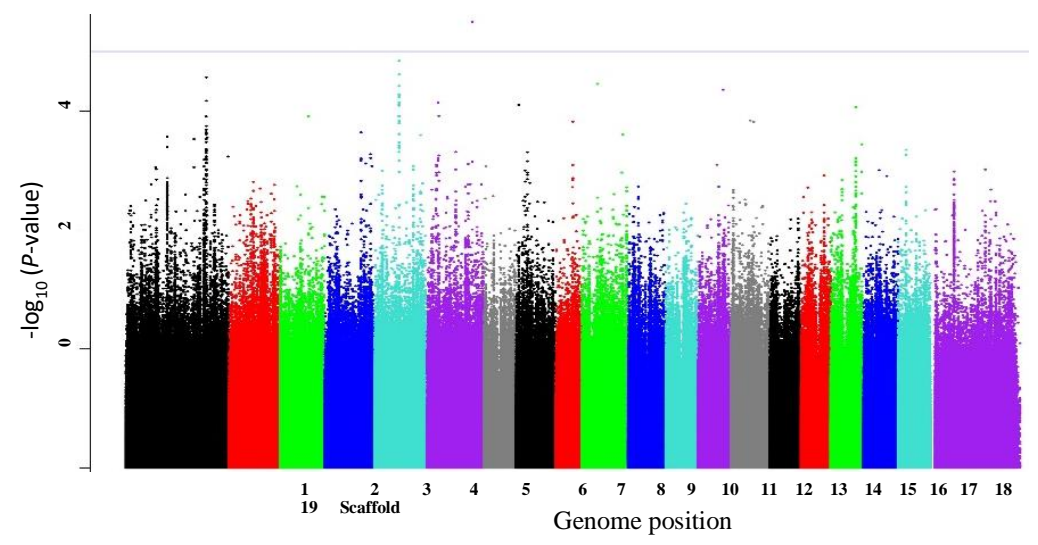

(e)

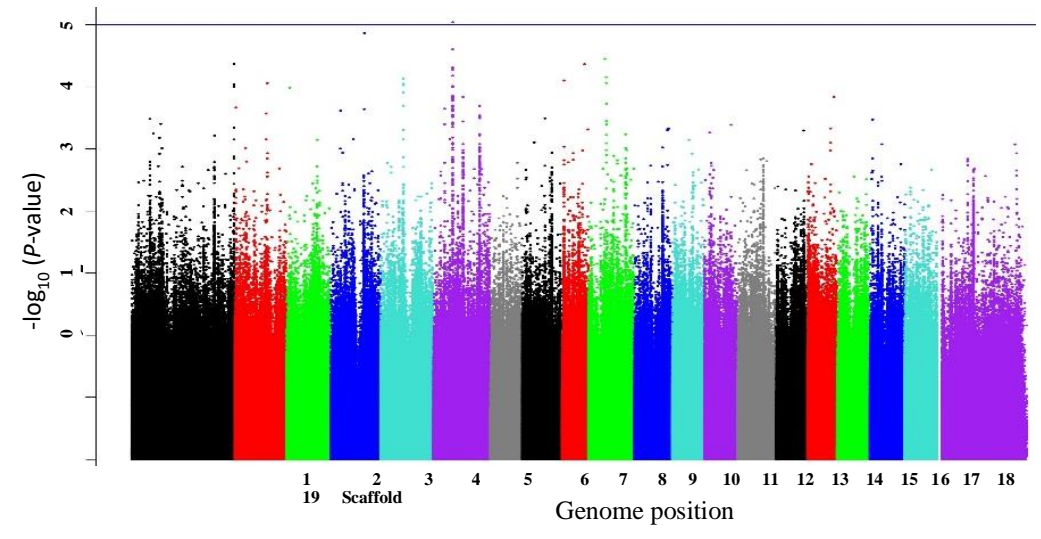

(b)

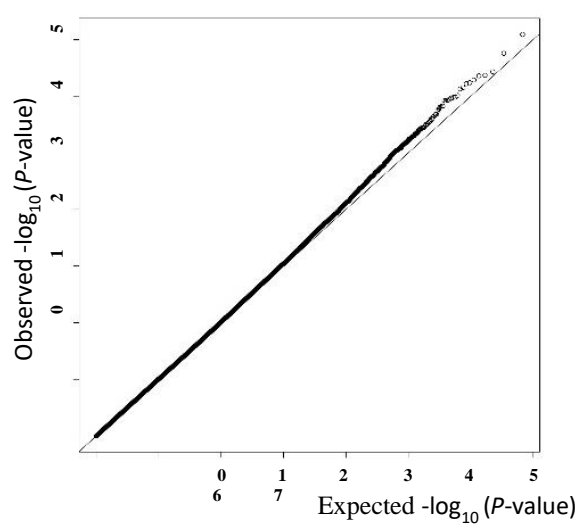

(d)

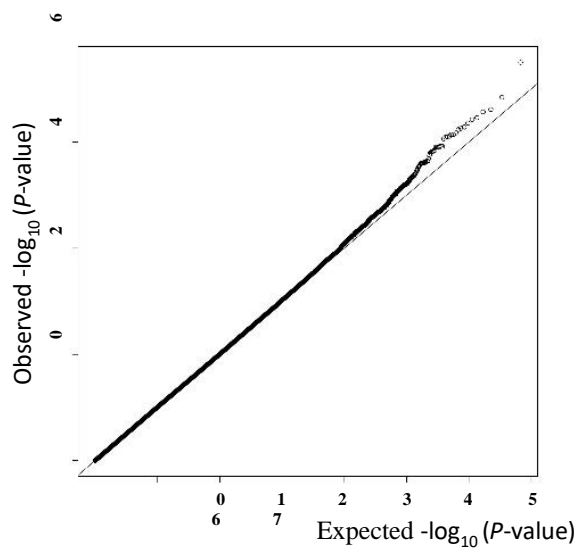

(f)

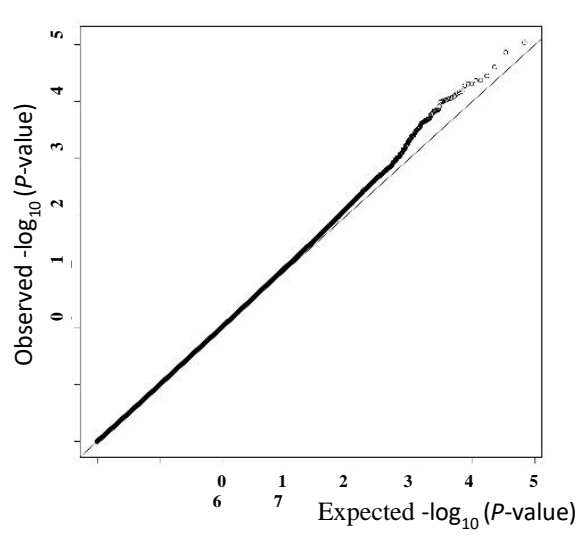


(g)

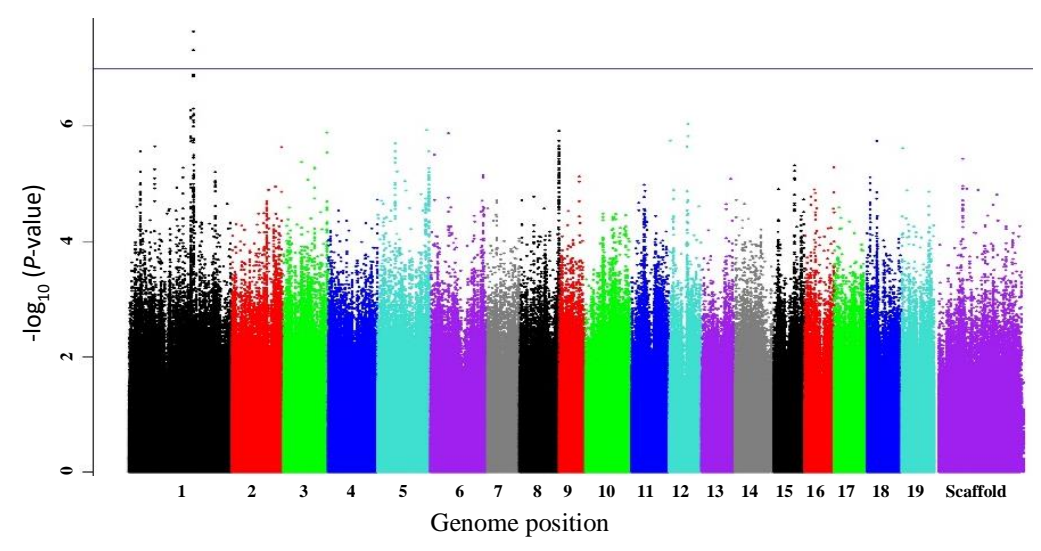

(h)

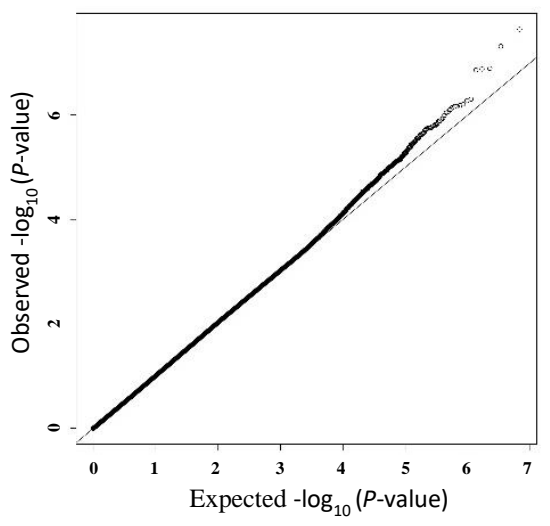

Supplementary Figure S 4.1 Single trait GWAS - Manhattan (left) and QQ plot (right). Numbers 1 to 19 represent chromosomes; scaffolds are the reads that did not align to any of the 19 chromosomes. (a) and (b) Intermediate wood vessel count; (c) and (d) Late wood vessel size; (e) and (f) S/G ratio; (g) and (h) Leaf aspect ratio. Red horizontal line indicates Bonferroni correction threshold $\left(P=7.417 \times 10^{-9}\right)$ and blue horizontal line indicates suggestive association threshold $\left(P=1 \times 10^{-7}\right)$.

(a)

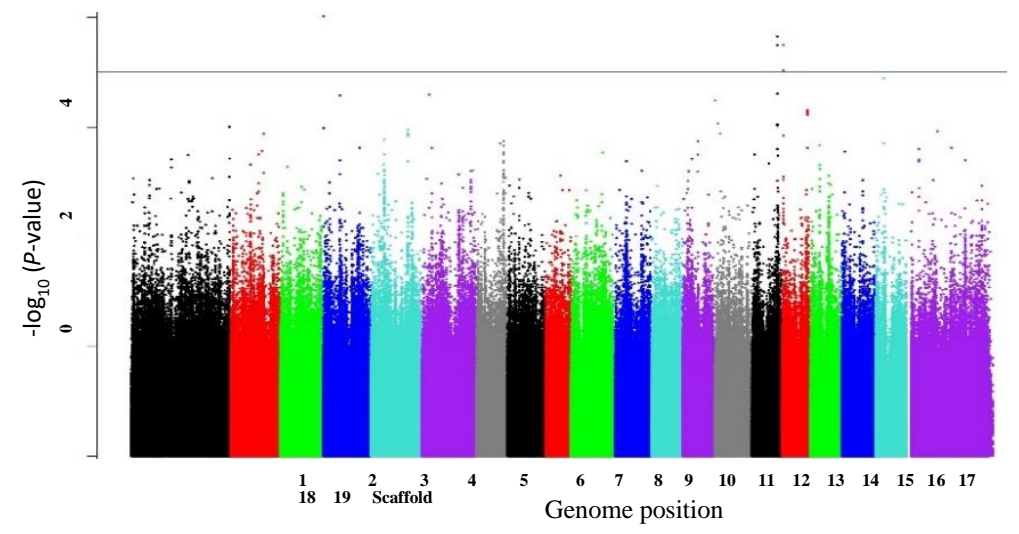

(b)

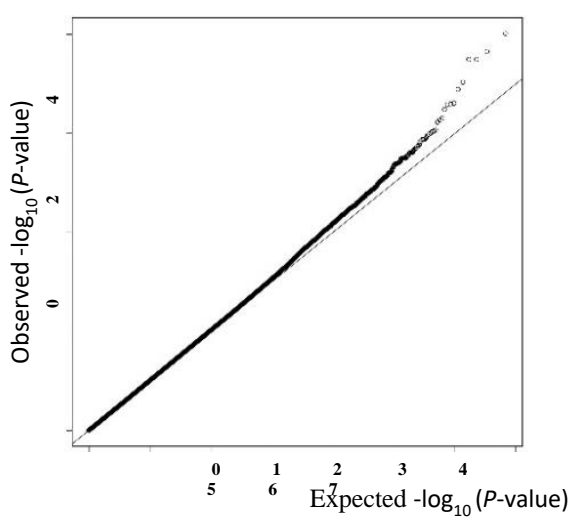


(c)

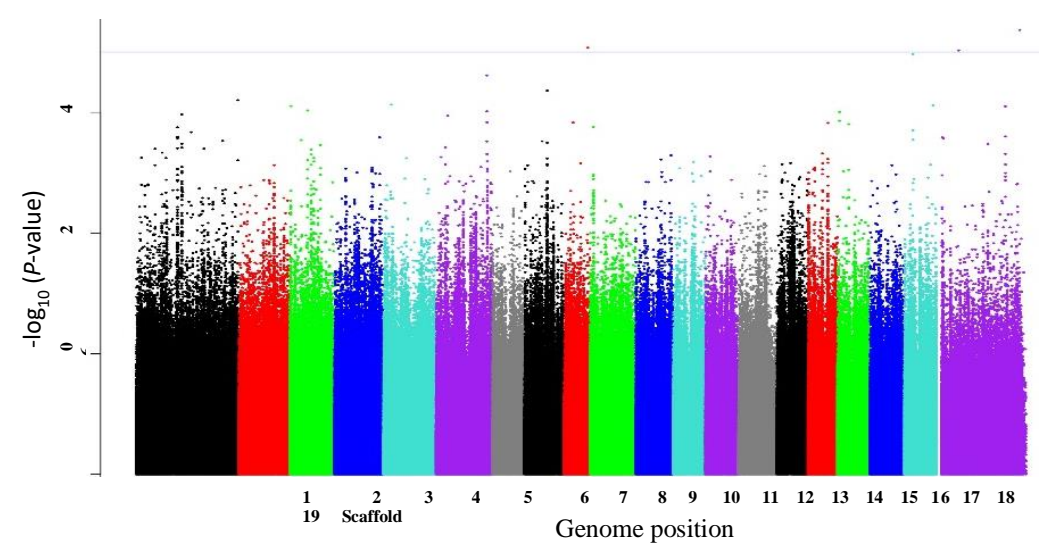

(e)

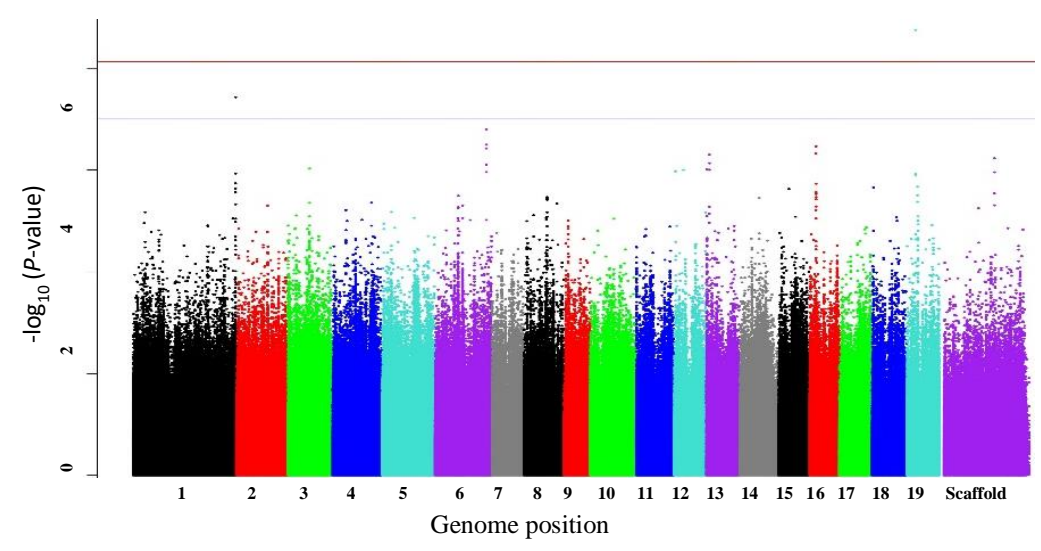

(g)

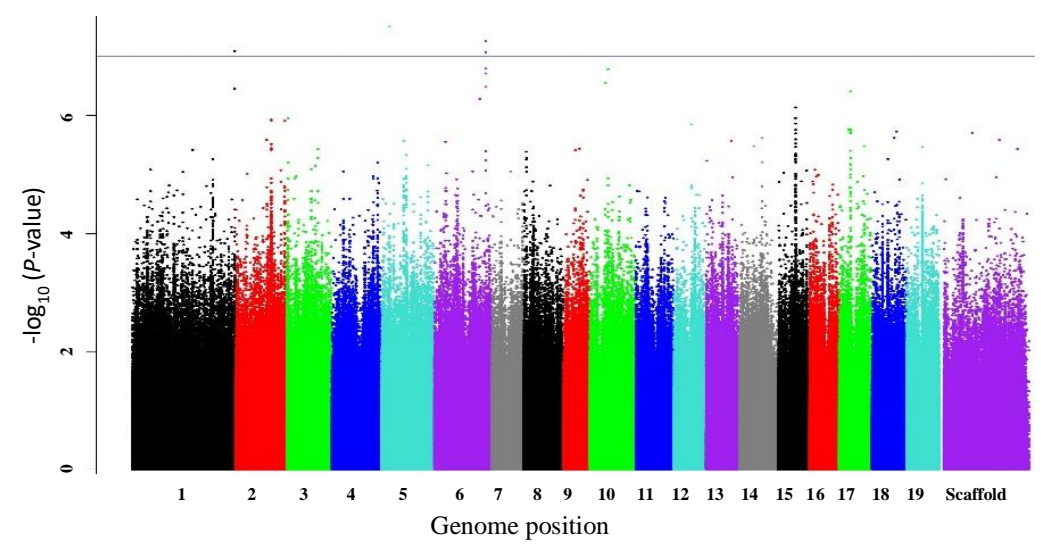

(d)

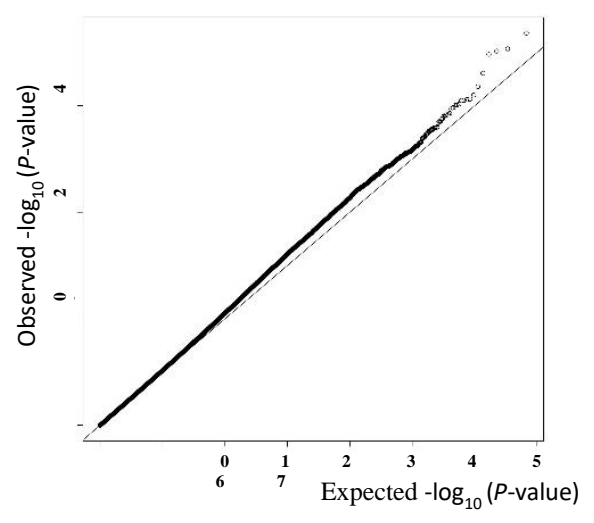

(f)

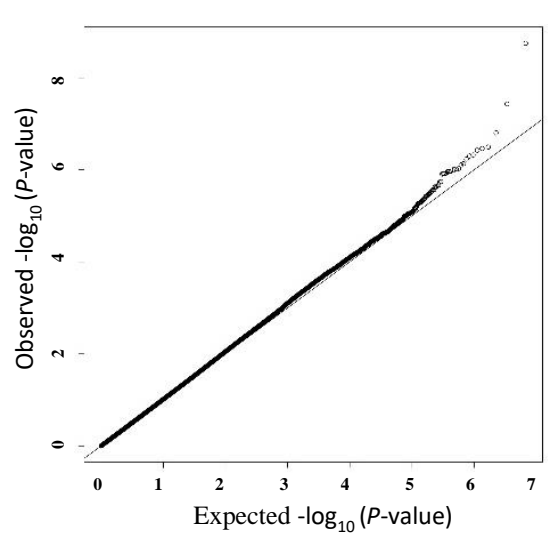

(h)

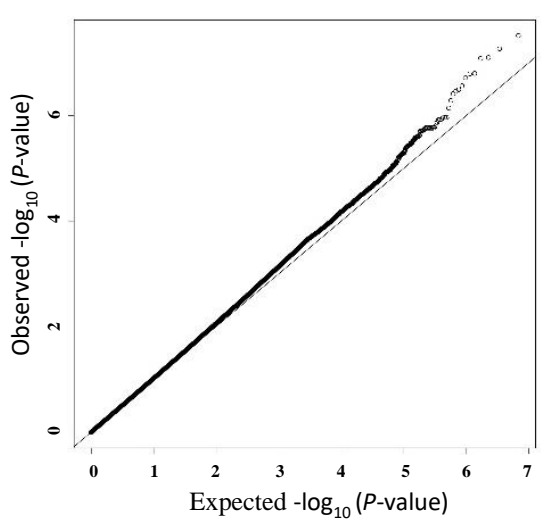


(i)

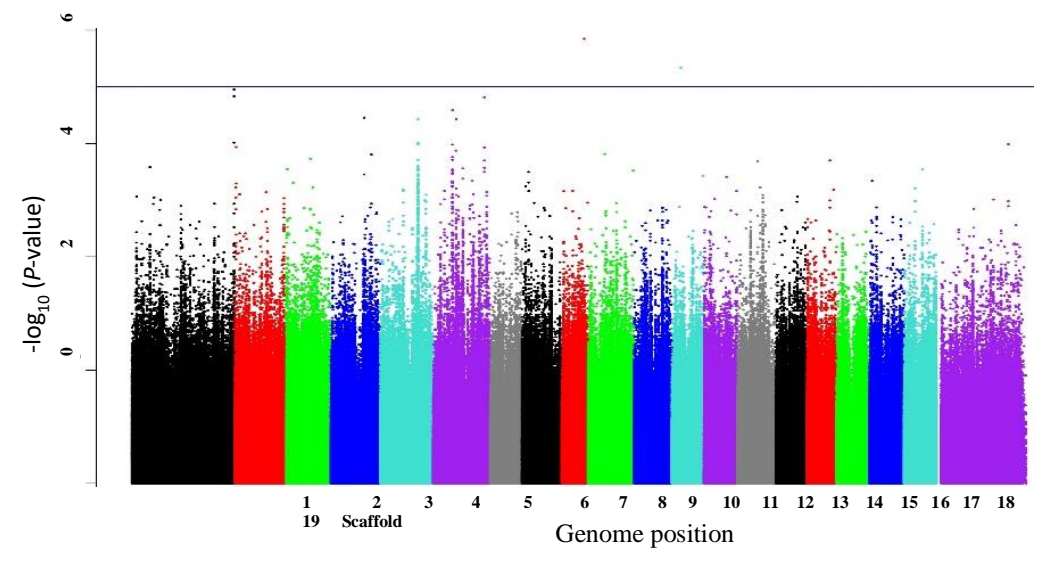

(k)

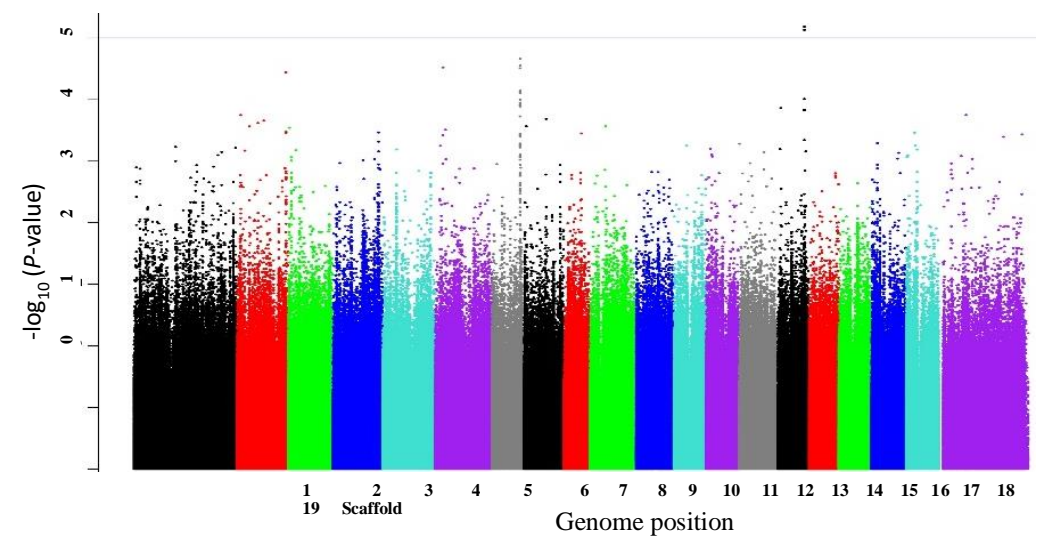

(m)

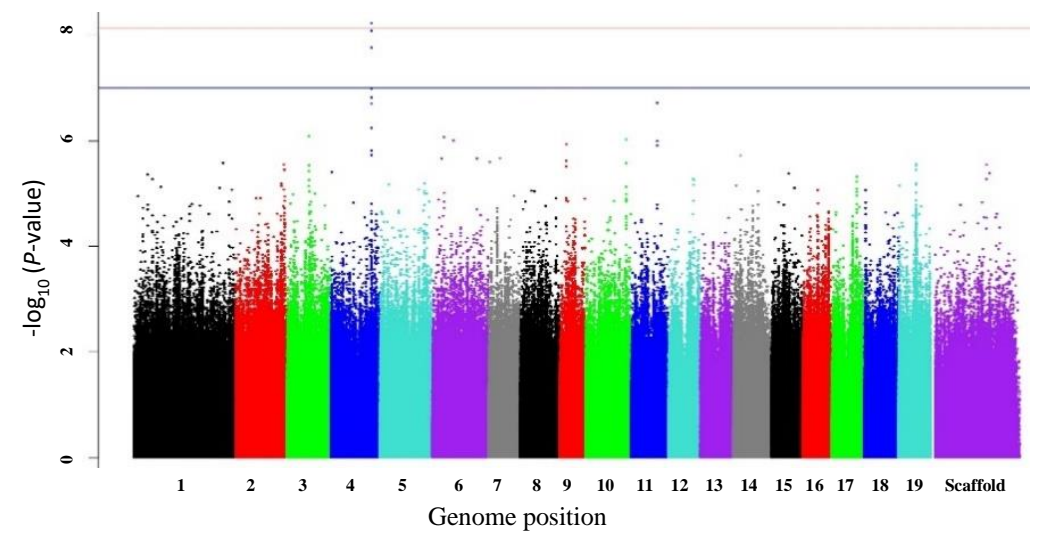

(j)

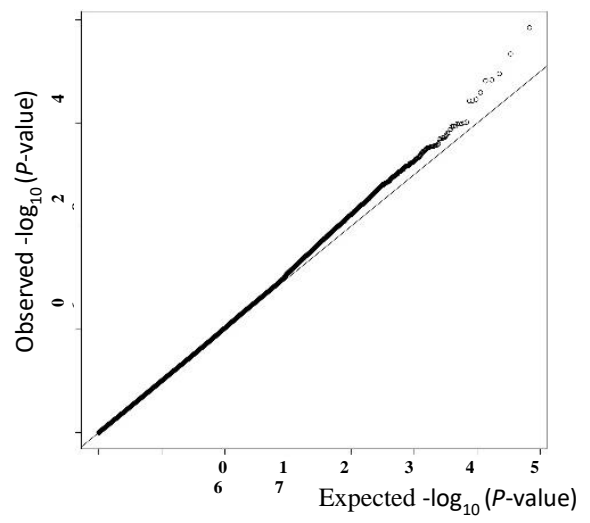

(1)

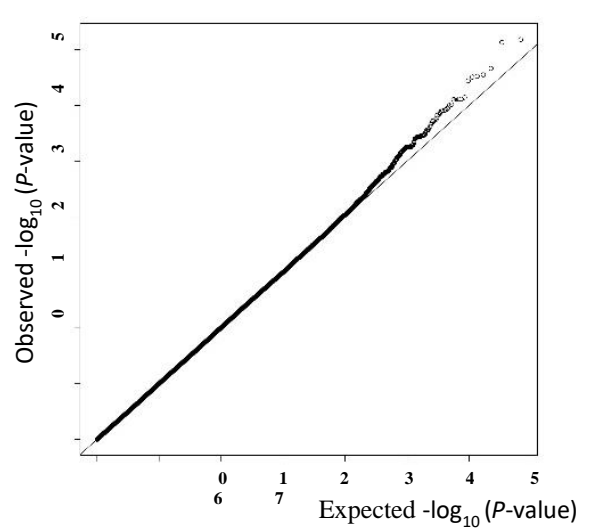

(n)

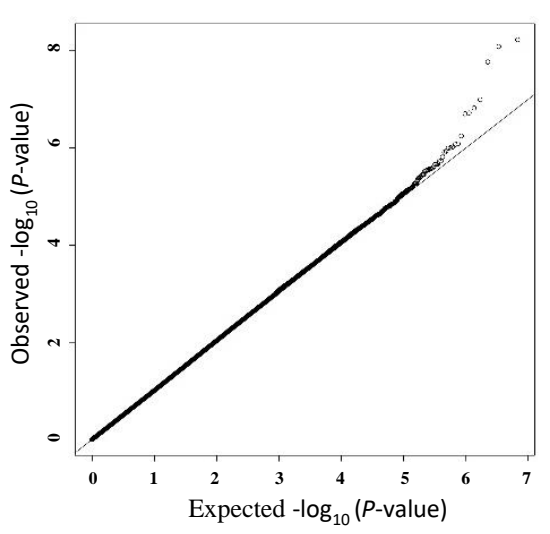


Supplementary Figure S 4.2 Multitrait GWAS - Manhattan (left) and QQ plot (right). Numbers 1 to 19 represent chromosomes; scaffolds are the reads that did not align to any of the 19 chromosomes. (a) and (b) Early wood vessel area, leaf area and stomatal density; (c) and (d) Early wood vessel size, intermediate wood vessel size, lignin content, S/G ratio and late wood vessel size; (e) and (f) Intermediate wood vessel size, lignin content and S/G ratio; (g) and (h) Lignin content, late wood vessel size and S/G ratio; (i) and (j) Lignin content and S/G ratio; (k) and (1) Lignin content, specific leaf area, stomatal density and early wood vessel area; (m) and (n) Late wood vessel area and late wood vessel count. Red horizontal line indicates Bonferroni correction threshold $\left(P=7.417 \times 10^{-9}\right)$ and blue horizontal line indicates suggestive association threshold $\left(P=1 \times 10^{-7}\right)$. 


\subsection{References}

Afzal, A. J., Wood, A. J., \& Lightfoot, D. A. (2008). Plant Receptor-Like Serine Threonine Kinases: Roles in Signaling and Plant Defense. Molecular Plant-Microbe Interactions, 21(5), 507-517. doi: 10.1094/mpmi-21-5-0507

Allwright, M. R., Payne, A., Emiliani, G., Milner, S., Viger, M., Rouse, F., ... Taylor, G. (2016). Biomass traits and candidate genes for bioenergy revealed through association genetics in coppiced European Populus nigra (L.). Biotechnology for Biofuels, 9(1), 1-22. doi: 10.1186/s13068-016-0603-1

Barakat, A., Szick-miranda, K., Chang, I., Guyot, R., Blanc, G., Cooke, R., ... Bailey-serres, J. (2001). The Organization of Cytoplasmic Ribosomal Protein Genes in the Arabidopis Genome. Plant Physio, 127, 398-415. doi: 10.1104/pp.010265.398

Bdeir, R., Muchero, W., Yordanov, Y., Tuskan, G. A., Busov, V., \& Gailing, O. (2019). Genome-wide association studies of bark texture in Populus trichocarpa. Tree Genetics and Genomes, 15(1). doi: $10.1007 / \mathrm{s} 11295-019-1320-2$

Bhavsar, R. B., Makley, L. N., \& Tsonis, P. A. (2010). The other lives of ribosomal proteins. Human Genomics, 4(5), 327-344. doi: 10.1186/1479-7364-4-5-327

Biswal, A. K., Atmodjo, M. A., Pattathil, S., Amos, R. A., Yang, X., Winkeler, K., ... Mohnen, D. (2018). Working towards recalcitrance mechanisms: Increased xylan and homogalacturonan production by overexpression of GAlactUronosylTransferase 12 (GAUT12) causes increased recalcitrance and decreased growth in Populus. Biotechnology for Biofuels, 11(1), 1-26. doi: 10.1186/s13068-017-1002-y

Boyle, E. A., Li, Y. I., \& Pritchard, J. K. (2017). An Expanded View of Complex Traits: From Polygenic to Omnigenic. Cell, 169(7), 1177-1186. doi: 10.1016/j.cell.2017.05.038

Caffall, K. H., Pattathil, S., Phillips, S. E., Hahn, M. G., \& Mohnen, D. (2009). Arabidopsis thaliana TDNA mutants implicate GAUT genes in the biosynthesis of pectin and xylan in cell walls and seed testa. Molecular Plant, 2(5), 1000-1014. doi: 10.1093/mp/ssp062

Carlquist, S. (2012). How wood evolves: a new synthesis. Botany, 90(10), 901-940. doi: 10.1139/b2012048

Chhetri, H. B., Macaya-sanz, D., Kainer, D., Biswal, A. K., Evans, L. M., Chen, J., ... Difazio, S. P. (2019). Multitrait genome-wide association analysis of Populus trichocarpa identifies key polymorphisms controlling morphological and physiological traits. New Phytologist, 223, 293-309. doi: 10.1111/nph.15777

Devis, D., Firth, S. M., Liang, Z., \& Byrne, M. E. (2015). Dosage Sensitivity of RPL9 and Concerted Evolution of Ribosomal Protein Genes in Plants. Frontiers in Plant Science, 6(December), 1-12. doi: 10.3389/fpls.2015.01102 
Du, Q., Gong, C., Wang, Q., Zhou, D., Yang, H., Pan, W., ... Zhang, D. (2016). Genetic architecture of growth traits in Populus revealed by integrated quantitative trait locus (QTL) analysis and association studies. New Phytologist, 209(3), 1067-1082. doi: 10.1111/nph.13695

Du, Q., Lu, W., Quan, M., Xiao, L., Song, F., Li, P., ... Zhang, D. (2018). Genome-Wide Association Studies to Improve Wood Properties: Challenges and Prospects. Frontiers in Plant Science, 9(December), 1-10. doi: 10.3389/fpls.2018.01912

Du, Q., Pan, W., Xu, B., Li, B., \& Zhang, D. (2013). Polymorphic simple sequence repeat (SSR) loci within cellulose synthase (PtoCesA) genes are associated with growth and wood properties in Populus tomentosa. New Phytologist, 197(3), 763-776. doi: 10.1111/nph.12072

Du, Q., Yang, X., Xie, J., Quan, M., Xiao, L., Lu, W., ... Zhang, D. (2019). Time-specific and pleiotropic quantitative trait loci coordinately modulate stem growth in Populus. Plant Biotechnology Journal, 17(3), 608-624. doi: 10.1111/pbi.13002

Eamens, A. L., Kim, K. W., Curtin, S. J., \& Waterhouse, P. M. (2012). DRB2 is required for microRNA biogenesis in Arabidopsis thaliana. PLoS ONE, 7(4). doi: 10.1371/journal.pone.0035933

Eamens, A. L., Smith, N. A., Curtin, S. J., Wang, M. B., \& Waterhouse, P. M. (2009). The Arabidopsis thaliana double-stranded RNA binding protein DRB1 directs guide strand selection from microRNA duplexes. Rna, 15(12), 2219-2235. doi: 10.1261/rna.1646909

Escamez, S., Latha Gandla, M., Derba-Maceluch, M., Lundqvist, S. O., Mellerowicz, E. J., Jönsson, L. J., \& Tuominen, H. (2017). A collection of genetically engineered Populus trees reveals wood biomass traits that predict glucose yield from enzymatic hydrolysis. Scientific Reports, 7(1), 1-11. doi: $10.1038 / \mathrm{s} 41598-017-16013-0$

Evans, L. M., Slavov, G. T., Rodgers-Melnick, E., Martin, J., Ranjan, P., Muchero, W., ... DiFazio, S. P. (2014). Population genomics of Populus trichocarpa identifies signatures of selection and adaptive trait associations. Nature Genetics, 46(10), 1089-1096. doi: 10.1038/ng.3075

Fahrenkrog, A. M., Neves, L. G., Resende, M. F. R., Dervinis, C., Davenport, R., Barbazuk, W. B., \& Kirst, M. (2017). Population genomics of the eastern cottonwood (Populus deltoides). Ecology and Evolution, 7(22), 9426-9440. doi: 10.1002/ece3.3466

Gandla, M. L., Martín, C., \& Jönsson, L. J. (2018). Analytical Enzymatic Saccharification of Lignocellulosic Biomass for Conversion to Biofuels and Bio-Based Chemicals. Energies, 11(11). doi: 10.3390/en11112936

González-Martínez, S. C., Ersoz, E., Brown, G. R., Wheeler, N. C., \& Neale, D. B. (2006). DNA sequence variation and selection of tag single-nucleotide polymorphisms at candidate genes for drought-stress response in Pinus taeda L. Genetics, 172(3), 1915-1926. doi: 10.1534/genetics.105.047126 
Goodstein, D. M., Shu, S., Howson, R., Neupane, R., Hayes, R. D., Fazo, J., ... Rokhsar, D. S. (2012). Phytozome: A comparative platform for green plant genomics. Nucleic Acids Research, 40(D1), 1178-1186. doi: 10.1093/nar/gkr944

Groover, A. T., Nieminen, K., Helariutta, Y., \& Mansfield, S. D. (2010). Wood Formation in Populus. In S. Jansson, Ri. P. Bhalerao, \& A. T. Groover (Eds.), Genetics and Genomics of Populus (pp. 201224). Springer.

Gudesblat, G. E., Schneider-Pizoń, J., Betti, C., Mayerhofer, J., Vanhoutte, I., Van Dongen, W., ... Russinova, E. (2012). SPEECHLESS integrates brassinosteroid and stomata signalling pathways. Nature Cell Biology, 14(5), 548-554. doi: 10.1038/ncb2471

Hansen, S. F., Harholt, J., Oikawa, A., \& Scheller, H. V. (2012). Plant Glycosyltransferases Beyond CAZy: A Perspective on DUF Families. Frontiers in Plant Science, 3(March), 1-10. doi: 10.3389/fpls.2012.00059

Hietz, P., Rosner, S., Hietz-Seifert, U., \& Wright, S. J. (2017). Wood traits related to size and life history of trees in a Panamanian rainforest. New Phytologist, 213(1), 170-180. doi: 10.1111/nph.14123

Ingvarsson, P. K., Hvidsten, T. R., \& Street, N. R. (2016). Towards integration of population and comparative genomics in forest trees. New Phytologist, 212(2), 338-344. doi: 10.1111/nph.14153

Johnson, A. M., Kim, H., Ralph, J., \& Mansfield, S. D. (2017). Natural acetylation impacts carbohydrate recovery during deconstruction of Populus trichocarpa wood. Biotechnology for Biofuels, 10(1), 112. doi: 10.1186/s13068-017-0734-z

Khan, M., Rozhon, W., Bigeard, J., Pflieger, D., Husar, S., Pitzschke, A., ... Poppenberger, B. (2013). Brassinosteroid-regulated GSK3/Shaggy-like kinases phosphorylate mitogen-activated protein (MAP) kinase kinases, which control stomata development in Arabidopsis thaliana. Journal of Biological Chemistry, 288(11), 7519-7527. doi: 10.1074/jbc.M112.384453

Lachenbruch, B., Moore, J. R., \& Evans, R. (2011). Radial Variation in Wood Structure and Function in Woody Plants, and Hypotheses for its Occurence. In F. C. Meinzer, B. Lachenbruch, \& T. E. Dawson (Eds.), Size and Age Related Changes in Tree Structure and Function (pp. 121-164). Springer.

Mckown, A. D., Guy, R. D., Klapste, J., Geraldes, A., Friedmann, M., Cronk, Q. C. B., ... Douglas, C. J. (2014). Geographical and environmental gradients shape phenotypic trait variation and genetic structure in Populus trichocarpa. New Phytologist, 201(4), 1263-1276. doi: 10.1111/nph.12601

McKown, A. D., Guy, R. D., Quamme, L., Klápště, J., La Mantia, J., Constabel, C. P., ... Azam, M. S. (2014). Association genetics, geography and ecophysiology link stomatal patterning in Populus trichocarpa with carbon gain and disease resistance trade-offs. Molecular Ecology, 23(23), 57715790. doi: 10.1111/mec.12969 
McKown, A. D., Klápště, J., Guy, R. D., Geraldes, A., Porth, I., Hannemann, J., ... Douglas, C. J. (2014). Genome-wide association implicates numerous genes underlying ecological trait variation in natural populations of Populus trichocarpa. New Phytologist, 203(2), 535-553. doi: 10.1111/nph.12815

Moura, J. C. M. S., Bonine, C. A. V., de Oliveira Fernandes Viana, J., Dornelas, M. C., \& Mazzafera, P. (2010). Abiotic and biotic stresses and changes in the lignin content and composition in plants. Journal of Integrative Plant Biology, 52(4), 360-376. doi: 10.1111/j.1744-7909.2010.00892.x

Muchero, W., Guo, J., DiFazio, S. P., Chen, J., Ranjan, P., Slavov, G. T., ... Tuskan, G. A. (2015). Highresolution genetic mapping of allelic variants associated with cell wall chemistry in Populus. BMC Genomics, 16, 24. doi: 10.1186/s12864-015-1215-Z

Muchero, W., Sondreli, K. L., Chen, J.-G., Urbanowicz, B. R., Zhang, J., Singan, V., .. LeBoldus, J. M. (2018). Association mapping, transcriptomics, and transient expression identify candidate genes mediating plant-pathogen interactions in a tree. Proceedings of the National Academy of Sciences, 115(45), 11573-11578. doi: 10.1073/pnas.1804428115

Neale, D. B., \& Kremer, A. (2011). Forest tree genomics: Growing resources and applications. Nature Reviews Genetics, 12(2), 111-122. doi: 10.1038/nrg2931

Neale, D. B., \& Savolainen, O. (2004). Association genetics of complex traits in conifers. New Phytologist, 9(7), 325-330. doi: 10.1111/j.1469-8137.2010.03593.x

Orfila, C., Sørensen, S. O., Harholt, J., Geshi, N., Crombie, H., Truong, H. N., ... Scheller, H. V. (2005). QUASIMODO1 is expressed in vascular tissue of Arabidopsis thaliana inflorescence stems, and affects homogalacturonan and xylan biosynthesis. Planta, 222(4), 613-622. doi: 10.1007/s00425005-0008-z

Porter, H. F., \& O’Reilly, P. F. (2017). Multivariate simulation framework reveals performance of multitrait GWAS methods. Scientific Reports, 7(May 2016), 1-12. doi: 10.1038/srep38837

Porth, I., Klápště, J., McKown, A. D., La Mantia, J., Guy, R. D., Ingvarsson, P. K., ... El-Kassaby, Y. A. (2015). Evolutionary Quantitative Genomics of Populus trichocarpa. PLOS ONE, 10(11), e0142864. doi: 10.1371/journal.pone.0142864

Porth, I., Klápště, J., Skyba, O., Friedmann, M. C., Hannemann, J., Ehlting, J., ... Douglas, C. J. (2013). Network analysis reveals the relationship among wood properties, gene expression levels and genotypes of natural Populus trichocarpa accessions. New Phytologist, 200(3), 727-742. doi: 10.1111/nph.12419

Porth, I., Ranjan, P., Klapšte, J., Guy, R. D., Tuskan, G. A., Ehlting, J., ... Hannemann, J. (2013). Genome-wide association mapping for wood characteristics in Populus identifies an array of candidate single nucleotide polymorphisms. New Phytologist. doi: 10.1111/nph.12422

Preston, K. A., Cornwell, W. K., \& DeNoyer, J. L. (2006). Wood density and vessel traits as distinct 
correlates of ecological strategy in 51 California coast range angiosperms. New Phytologist, 170(4), 807-818. doi: 10.1111/j.1469-8137.2006.01712.x

Ranty, B., Aldon, D., Cotelle, V., Galaud, J.-P., Thuleau, P., \& Mazars, C. (2016). Calcium Sensors as Key Hubs in Plant Responses to Biotic and Abiotic Stresses. Frontiers in Plant Science, 7(March), 1-7. doi: 10.3389/fpls.2016.00327

Schindelin, J., Rueden, C. T., Hiner, M. C., \& Eliceiri, K. W. (2015). The ImageJ ecosystem: An open platform for biomedical image analysis. Molecular Reproduction and Development, 82(7-8), 518529. doi: $10.1002 / \mathrm{mrd} .22489$

Shim, H., Chasman, D. I., Smith, J. D., Mora, S., Ridker, P. M., Nickerson, D. A., ... Stephens, M. (2015). A multivariate genome-wide association analysis of 10 LDL subfractions, and their response to statin treatment, in 1868 Caucasians. PLoS ONE, 10(4), 1-20. doi: 10.1371/journal.pone.0120758

Slavov, G. T., Difazio, S. P., Martin, J., Schackwitz, W., Muchero, W., Rodgers-Melnick, E., ... Tuskan, G. A. (2012). Genome resequencing reveals multiscale geographic structure and extensive linkage disequilibrium in the forest tree Populus trichocarpa. New Phytologist, 196(3), 713-725. doi: 10.1111/j.1469-8137.2012.04258.x

Sperry, J. S. (2003). Evolution of Water Transport and Xylem Structure. International Journal of Plant Sciences, 164(S3), S115-S127. doi: 10.1086/368398

Street, N. R., \& Ingvarsson, P. K. (2011). Association genetics of complex traits in plants. New Phytologist, 189, 909-922. doi: 10.1111/j.1469-8137.2010.03593.x

Studer, M. H., DeMartini, J. D., Davis, M. F., Sykes, R. W., Davison, B., Keller, M., ... Wyman, C. E. (2011). Lignin content in natural Populus variants affects sugar release. Proceedings of the National Academy of Sciences, 108(15), 6300-6305. doi: 10.1073/pnas.1009252108

Verger, S., Chabout, S., Gineau, E., \& Mouille, G. (2016). Cell adhesion in plants is under the control of putative O-fucosyltransferases. Journal of Cell Science, 129(15), e1.2-e1.2. doi: 10.1242/jcs.195537

Visscher, P. M., Wray, N. R., Zhang, Q., Sklar, P., McCarthy, M. I., Brown, M. A., \& Yang, J. (2017). 10 Years of GWAS Discovery: Biology, Function, and Translation. American Journal of Human Genetics, 101(1), 5-22. doi: 10.1016/j.ajhg.2017.06.005

Wegrzyn, J. L., Eckert, A. J., Choi, M., Lee, J. M., Stanton, B. J., Sykes, R., ... Neale, D. B. (2010). Association genetics of traits controlling lignin and cellulose biosynthesis in black cottonwood (Populus trichocarpa, Salicaceae) secondary xylem. New Phytologist, 188(2), 515-532. doi: 10.1111/j.1469-8137.2010.03415.x

Weighill, D., Jones, P., Bleker, C., Ranjan, P., Shah, M., Zhao, N., .. Jacobson, D. (2019). MultiPhenotype Association Decomposition: Unraveling Complex Gene-Phenotype Relationships. Frontiers in Genetics, 10(May), 1-17. doi: 10.3389/fgene.2019.00417 
Weighill, D., Jones, P., Shah, M., Ranjan, P., Muchero, W., Schmutz, J., ... Jacobson, D. (2018).

Pleiotropic and Epistatic Network-Based Discovery: Integrated Networks for Target Gene

Discovery. Frontiers in Energy Research, 6(May), 30. doi: 10.3389/fenrg.2018.00030

Xi, W., Song, D., Sun, J., Shen, J., \& Li, L. (2017). Formation of wood secondary cell wall may involve two type cellulose synthase complexes in Populus. Plant Molecular Biology, 93(4-5), 419-429. doi: $10.1007 / \mathrm{s} 11103-016-0570-8$

Xie, M., Zhang, J., Tschaplinski, T. J., Tuskan, G. A., Chen, J.-G., \& Muchero, W. (2018). Regulation of Lignin Biosynthesis and Its Role in Growth-Defense Tradeoffs. Frontiers in Plant Science, 9(September), 1-9. doi: 10.3389/fpls.2018.01427

Zeng, H., Xu, L., Singh, A., Wang, H., Du, L., \& Poovaiah, B. W. (2015). Involvement of calmodulin and calmodulin-like proteins in plant responses to abiotic stresses. Frontiers in Plant Science, 6(August), 1-12. doi: 10.3389/fpls.2015.00600

Zentella, R., Sui, N., Barnhill, B., Hsieh, W. P., Hu, J., Shabanowitz, J., ... Sun, T. P. (2017). The Arabidopsis O-fucosyltransferase SPINDLY activates nuclear growth repressor della. Nature Chemical Biology, 13(5), 479-485. doi: 10.1038/nchembio.2320

Zentella, R., Zhang, Z.-L., Park, M., Thomas, S. G., Endo, A., Murase, K., ... Sun, T. (2007). Global Analysis of DELLA Direct Targets in Early Gibberellin Signaling in Arabidopsis. The Plant Cell, 19(10), 3037-3057. doi: 10.1105/tpc.107.054999

Zhang, J., Yang, Y., Zheng, K., Xie, M., Feng, K., Jawdy, S. S., ... Muchero, W. (2018). Genome-wide association studies and expression-based quantitative trait loci analyses reveal roles of HCT2 in caffeoylquinic acid biosynthesis and its regulation by defense-responsive transcription factors in Populus. New Phytologist, 220(2), 502-516. doi: 10.1111/nph.15297

Zhou, X., \& Stephens, M. (2012). Genome-wide efficient mixed-model analysis for association studies. Nature Genetics, 44(7), 821-826. doi: 10.1038/ng.2310

Zhou, X., \& Stephens, M. (2014). Efficient multivariate linear mixed model algorithms for genome- wide association studies. Nature Genetics, 11(4), 407-411. doi: 10.1038/nmeth.2848 


\section{Chapter 5. Overall conclusion}

One of the major goals in population genomics is to understand the role of evolutionary forces such as selection, mutation and gene flow in shaping the spatial and temporal patterns of phenotypic variation. With the revolution in sequencing technology, it is now possible to assess the molecular mechanisms underlying phenotypic variation in adaptive traits. The identified genetic targets have great potential for optimizing traits related to abiotic stress tolerance and improving productivity. The genomic revolution has also enabled the identification of underlying genetic mechanisms affecting local adaptation, leading to better understanding of the biology of the species in the context of rapidly changing climates (Aitken, Yeaman, Holliday, Wang, \& Curtis-McLane, 2008). Because of their wide geographical distribution and climatic gradients, large effective population sizes, high genetic variation, forest trees are excellent model systems for understanding local adaptation and genetic architecture of the complex traits (González-Martínez, Ersoz, Brown, Wheeler, \& Neale, 2006; Ingvarsson, Hvidsten, \& Street, 2016; Neale \& Kremer, 2011; Neale \& Savolainen, 2004; Street \& Ingvarsson, 2011).

Efforts have been made to optimize this ecologically and economically important tree Populus for lignocellulosic biofuel production using association genetics by identifying the genomic regions underlying traits of interest (Street \& Ingvarsson, 2011). Using natural populations that have undergone many generations of recombination between ancestral haplotypes allows identification of the genomic region affecting a trait at fine scale. To this end, several small and some large scale GWAS studies have been performed to understand the underlying genetic architecture related to morphological, physiological, wood chemistry and disease resistance traits in Populus (Allwright et al., 2016; Bdeir et al., 2019; Du et al., 2019; Evans et al., 2014; Fahrenkrog et al., 2017; McKown, Klápště, et al., 2014; Muchero et al., 2015; Porth et al., 2015, 2013; Wegrzyn et al., 2010; Zhang et al., 2018). However, the comprehensive assessment of morphological and physiological traits comparing GWAS from multiple plantations is very rare (Evans et al., 2014). Furthermore, there is no GWAS study on wood anatomical traits such as vessel size and density that is related to wood chemistry and important plant functions. My dissertation provides a comprehensive assessment of GWAS on important wood anatomical, wood chemistry, morphological and physiological traits in Populus trichocarpa. I also assess the effect of environment on GWAS for morphological traits measured in two contrasting common gardens. Furthermore, I complemented phenotypic GWAS with the genomics of local adaptation to understand the molecular genetic mechanisms underlying local adaptation in $P$. trichocarpa.

In chapter 2, I performed 14 single and 12 multitrait GWAS on morphological and physiological data collected from a common garden in Corvallis, OR. Using GEMMA software (Zhou \& Stephens, 2012, 2014), I tested for the association of these traits with 6.78 million SNPs in the genome from 882 
trees. I created multitrait sets based on the correlation and functional relationships of the traits and showed that multitrait GWAS provided substantial increase in power over single trait GWAS for detecting the SNP variants.

Complex adaptive traits are not just affected by single or a few loci, and thousands of loci in the network with non-zero effect sizes affect the trait (Boyle, Li, \& Pritchard, 2017). Power analyses indicate that most association studies in forest trees are orders of magnitude too small to detect the effects of alleles of small effect and low frequency (Visscher et al., 2017). Therefore, despite the high heritability of many morphological traits, only a small proportion of heritability is explained by single nucleotide polymorphisms (SNPs) in most GWAS analyses, suggesting insufficient statistical power (Solovieff, Cotsapas, Lee, Purcell, \& Smoller, 2013). While increasing sample size of GWAS populations is clearly desirable, it is costly and, in some cases, may not be feasible. Therefore, I used a multivariate framework, to increase the power of my GWAS study. The multitrait approach offers substantial increase in power compared to the standard univariate approach (Porter \& O'Reilly, 2017). One of the big advantages of multitrait GWAS is that missing information in one of the phenotypes in the multitrait set can be complemented by the other phenotypes (Ritchie, Holzinger, Li, Pendergrass, \& Kim, 2015). Multitrait GWAS also takes advantage of pleiotropic effects of polymorphisms, thereby increasing statistical power even when the traits have low correlation (Broadaway et al., 2016; Hackinger \& Zeggini, 2017). Finally, unlike analyses based on principal components, multitrait GWAS effectively captures indirect genetic effects whereby a SNP affects one phenotype through its effects on a functionally-related phenotype (Porter \& O'Reilly, 2017; Stephens, 2013).

In chapter 2, I identified 5 SNPs that passed the Bonferroni correction $P$-value cutoff of $7.37 \times 10^{-9}$ with multitrait GWAS for a total of 12 sets of traits compared to none with single trait GWAS for 14 traits. Furthermore, a total of 32 SNPs passed the suggestive association $P$-value cutoff of $1 \times 10^{-7}$ for multitrait GWAS compared to 4 SNPs for single traits GWAS. With multitrait GWAS I identified $22 P$. trichocarpa gene models, whereas with single trait GWAS I identified only 4 gene models. For single trait GWAS, no SNP passed Bonferroni correction threshold and only 4 SNPs passed the suggestive association threshold. To gain further insight into possible functions of candidate genes identified by GWAS analysis, I examined the position of the genes in networks constructed from RNA-seq expression data and metabolites profiles for the same population. Functional characterization of one of the genes highlighted by this analysis was Potri.004G111000, identified from the multitrait GWAS for LA-LD-LLLW. GAUT9 belongs to the GAUT gene family of proven and putative pectin homogalacturonan (HG) galacturnosyltransferases (Atmodjo et al., 2011; Biswal et al., 2018; Sterling et al., 2006; Voiniciuc et al., 2018). Multiple lines of evidence showed that this gene can affect leaf development. GAUT9 had reciprocal effects on leaf size - leaf size increased on knockdown lines. 
Another notable gene I identified was for multitrait GWAS of CI-LA-SD where I identified EFHand Calcium Binding Domain. Calcium is an important second messenger in eukaryotes and has important roles in cell signaling and response to biotic and abiotic stresses and developmental cues (Edel, Marchadier, Brownlee, Kudla, \& Hetherington, 2017; Ranty et al., 2016; Zhu, 2016). The EF-hand motif is the most common and highly conserved calcium-binding motif (Lewit-Bentley \& Réty, 2000; Zeng, Zhang, Zhang, Pi, \& Zhu, 2017). The co-expression network further provided the evidence that the gene Potri.001G411800, identified in multitrait GWAS is involved in responses to abiotic stress. It is coexpressed with a late embryogenesis abundant (LEA) hydroxyproline-rich glycoprotein (Potri.009G158900), a group that has a major role in responses to drought, salinity and, osmotic and temperature related stresses (Gao \& Lan, 2016; Magwanga et al., 2018). Potri.001G411800 is also associated with 10 different metabolites in the same population, including several that are related to plant development and stress responses. These findings have important implications for optimizing traits related to plant productivity and stress tolerance in the biotechnology experiment. Furthermore, the lines of evidence (LOE) approach taken here to identify coexpressed genes and metabolites provided further support to the genes identified by GWAS. In conclusion, using single trait GWAS complemented with a multitrait approach helps identify pleiotropic loci. Furthermore, the LOE approach adds confidence and reduces false positives in GWAS results.

In chapter 3, I tested for the evidence of local adaptation in $P$. trichocarpa using three conceptually different methods. First, I tested for the correlation of phenotypic traits with climate and geography variables. Several traits including bud set, bud flush, tree height and diameter, and leaf traits had significant correlation with climate and geography variables suggesting the role of climate and geography in shaping the variation and local adaptation of this species.

Second, I tested for the association of six environmental variables with 6.741 million SNPs in the genome for 869 genotypes using univariate as well as multivariate genotype-environment association (GEA) approach. Furthermore, I also performed GWAS of the first two PCs of 26 climate and geography variables with 6.741 million SNPs in the genome. I identified a total of 422 significant SNPs in vicinity of 67 genes conferring local adaptation based on a Bonferroni correction threshold of 5\% significance level $\left(P<7.417 \times 10^{-9}\right)$. Fourteen of these genes were shared across the three genotype-environment association (GEA) detection methods - single and multitrait and PC based associations. Using GEA methods I identified multiple important genes including Potri.010G079500 (a very long chain beta-ketoacyl-CoA synthase, the best match of this gene is KCS11 in Arabidopsis, potential involvement in cuticular wax biosynthesis), Potri.010G080200 (also a very long chain beta-ketoacyl-CoA synthase) and Potri.010G079600 (DNA damage repair protein) genes. 
Third, using the multivariate ordination method called redundancy analysis (RDA), I identified several genomic outlier loci conferring local adaptation in P. trichocarpa. Decomposing the variance in the SNP response matrix into the matrices of climate and geography, respectively using RDA (climate RDA model), I underscored the relative contribution of climate and geography on neutral population structure. Similarly, I decomposed the variation in phenotypic matrix (11 traits) into the matrices of SNP (113 SNP eigenvectors), climate (5 variables) and geography (space variables selected based on the stepwise regression of $3^{\text {rd }}$ degree polynomials for latitude and longitude) (phenotypic RDA model) and showed that the climate explained the highest amount of variation $(21.664 \%)$ among the predictor matrices suggesting the impact of climate variables on adaptive traits. Furthermore, I identified a total of 7807 and 16782 outlier genes from climate and phenotypic RDA models, respectively. Comparison of these gene models with 67 gene models identified from GEA methods at $P$-value cutoff of $7.417 \times 10^{-9}$ showed that 32 of them were shared across climate and phenotypic RDA and the GEA methods. This included a few interesting genes in chromosome 10 including Potri.010G079500. The Potri.010G079500 gene appears to be important for the alkene composition of cuticular wax in P. trichocarpa (GonzalesVigil, Hefer, von Loessl, La Mantia, \& Mansfield, 2017). The gene is downregulated in trees with low alkene composition. Non-alkene producing trees had reduced growth and higher disease susceptibility, emphasizing the adaptive importance of this trait (Gonzales-Vigil et al., 2017).

Because my SNP data set was large (>11.1 million SNPs at MAF cutoff of 0.01), I used an indirect approach for detecting the loci conferring local adaption. I used significant SNP PCs (eigenvectors) from the PCA analysis as a matrix of genetic data in the RDA analysis. I considered the top $0.1 \%$ of the SNPs from the tails of the distribution of the loadings from the significant SNP PCs as outlier loci. A total of 5 and 17 outlier SNP PCs were identified as outliers in the climate and phenotypic RDA models, respectively. Given that the adaptation in forest trees is polygenic such that selection can affect hundreds or thousands of loci, the large number of genes we identified here is not surprising (Boyle et al., 2017; Eckert et al., 2009; Evans et al., 2014; Holliday, Wang, \& Aitken, 2013). Furthermore, the use of powerful methods like RDA is important to identify loci especially with weak selection signals. Overall, this study provided important insights on the mechanisms of local adaptation in forest trees. The genomic adaptation signals identified here can have important implications in breeding and optimizing the traits of interest in domestication and natural management of Populus and other tree species. This can also aid in predicting the performance of a genotype in a new environment.

In chapter 4, I performed GWAS on phenotypic traits in a different common garden in Clatskanie, OR with contrasting environmental conditions compared to Corvallis, OR. The Clatskanie common garden is located close to the mouth of Columbia river and the Pacific Ocean whereas Corvallis common garden is located in an inland valley, and is not as wet as the Clatskanie site. My objective for 
chapter 4 was two-fold: 1) I tested for the effect of environment on the GWAS of comparable morphological traits such as leaf and stomatal density traits from Corvallis common garden; and 2) I performed GWAS on wood anatomy traits such as vessel size and density for the first time in Populus.

Genes underlying wood chemistry and functional genomics of wood property traits are fairly well studied (Muchero et al., 2015; Porth et al., 2015, 2013; Wegrzyn et al., 2010), but the genetic architecture underlying wood anatomical traits such as vessel size and density that are functionally related to cell wall composition and overall plant function is unknown. Using GEMMA univariate GWAS for 27 phenotypic traits including wood anatomical, wood chemistry and morphological traits with 6.741 million SNPs in the genome for 869 trees, I detected two SNPs that passed Bonferroni correction threshold of $P<7.417 \times 10^{-9}$ (both SNPs detected for stomatal density) and 77 SNPs that passed suggestive association threshold of $P<1 \times 10^{-7}$. These 77 SNPs were within or close to 20 P. trichocarpa gene models. As in chapter 2, I also formed 19 multitrait combinations based on the correlation and functional relationships of the traits and identified 9 SNPs that passed the Bonferroni correction $P$-value cutoff and 47 SNPs that passed the suggestive association $P$-value cutoff. These SNPs were within or close to $33 P$. trichocarpa gene models.

Correlation of phenotypic traits with latitude in chapter 3 shows that geography (latitudinal variation in particular) has a major role on adaptive trait variation in P. trichocarpa, which similar to other studies, obscures the actual between-trait and trait-climate relationships (Chhetri et al., 2019; Mckown et al., 2014). Low correlations between traits from different common gardens suggest that there is a large effect of environment on phenotypic traits. This is also reflected in the GWAS study such that despite the moderate heritabilities of most traits, the genes controlling phenotypic traits localized to completely different positions in this study compared to the GWAS performed for the phenotypic traits in Corvallis, OR common garden. Furthermore, the correlation of phenotypic traits with climate variables such as temperature and moisture and precipitation supported the evidence of local adaptation in $P$. trichocarpa.

Nevertheless, I was able to identify some related genes for some functionally related traits with the GWAS from two common gardens. The gene model Potri.017G090200 identified from wood anatomy multitrait 5 (late wood vessel area and count, SNP $P$-value $=7.32 \times 10^{-9}$ ) encodes calmodulin-binding protein-like protein. Calmodulin (CaM) and calmodulin-like proteins (CMLs) are one of the three main families of calcium (CA2+) sensor proteins in plants (Ranty et al., 2016). Calmodulin (CaM) and calmodulin-like proteins (CMLs) are well characterized in plants and are involved in regulating plant responses to abiotic stresses (Zeng et al., 2017). One of the calmodulin binding proteins, EF-hand motif that is involved in cell signaling, developmental processes and biotic and abiotic responses was detected in the multitrait GWAS from Corvallis, OR plantation that included carbon isotope, leaf area and stomatal 
density in the multitrait set (Chhetri et al., 2019). Here I identified a gene with similar role controlling wood anatomy traits. Wood anatomy traits have potential functional relationships with carbon isotope, leaf area and stomatal density.

The gene, Potri.015G117500, identified based on two GWAS hits for stomatal density that passed Bonferroni threshold of $P<7.417 \times 10^{-9}$, provided another case where a gene with a similar role was identified for the same trait. Potri.015G1 17500 encodes peptide-O-fucosyltransferase which belongs to the family of glycosyltransferases (Hansen, Harholt, Oikawa, \& Scheller, 2012; Vogt \& Jones, 2000). In Arabidopsis O-fucosyltransferase affects DELLA and associated regulators such as phytochromeinteracting-factor3 (PIF3) and PIF4 in the barasinosteroid- and light-signaling pathways (Zentella et al., 2007). McKown et al. (2014) identified a similar gene, Potri.004G059000, encoding BRASSINOSTEROID-INSENSITIVE 2, for stomatal density based on the GWAS on 34K SNP array data for $464 P$. trichocarpa trees. BRASSINOSTEROID-INSENSITIVE 2 may affect the brassinosteroid signaling pathway and the regulation of stomatal development (Gudesblat et al., 2012; Khan et al., 2013).

In chapter 4, I detected several interesting genes related to plant growth, development and defense mechanisms, but these genes should be verified with co-expression networks from RNA-seq data and metabolite profiles from the same population. Furthermore, the functional validation of all genes detected in chapter 2, 3 and 4 is necessary in order to target the gene in the biotechnology experiments to optimize important plant growth and development and abiotic stress tolerance traits. This will aid in the domestication of Populus trees for feedstock development. Moreover, GWAS methods such as gene and pathway-based methods and insertion deletion (indel) GWAS methods should be used, as the former provide more power and the latter is conceptually different and highlights the importance of indels in the genome for controlling complex traits. SNPs identified in GWAS are useful not only for identifying the genetic variants that can be targeted in the biotechnology experiment to optimize the traits of interest, but they can also be useful, together with other marker information available for the population in a genomic selection program to estimate breeding values from genotypic data.

As I have highlighted, the lack of power in GWAS and local adaptation studies is mainly due to sample size. Increasing sample size by homogeneously sampling from across the range of the distribution is always important for understanding the underlying genetics controlling complex traits. Moreover, powerful methods such as multitrait GWAS coupled with integration of multi-omic data should complement large sample size and compensate for lack of power. Furthermore, given that the local adaptation is believed to be polygenic and confounded by demographic history, multivariate methods like redundancy analysis (RDA) provide more power to detect weak selection signals. Lastly, future local adaptation studies should integrate various data types including phenotypic, genomic and environmental data whenever possible. 


\section{References}

Aitken, S. N., Yeaman, S., Holliday, J. A., Wang, T., \& Curtis-McLane, S. (2008). Adaptation, migration or extirpation: climate change outcomes for tree populations. Evolutionary Applications, 1(1), 95111. doi: 10.1111/j.1752-4571.2007.00013.x

Allwright, M. R., Payne, A., Emiliani, G., Milner, S., Viger, M., Rouse, F., ... Taylor, G. (2016). Biomass traits and candidate genes for bioenergy revealed through association genetics in coppiced European Populus nigra (L.). Biotechnology for Biofuels, 9(1), 1-22. doi: 10.1186/s13068-016-0603-1

Atmodjo, M. A., Sakuragi, Y., Zhu, X., Burrell, A. J., Mohanty, S. S., Atwood, J. A., ... Mohnen, D. (2011). Galacturonosyltransferase (GAUT)1 and GAUT7 are the core of a plant cell wall pectin biosynthetic homogalacturonan:galacturonosyltransferase complex. Proceedings of the National Academy of Sciences, 108(50), 20225-20230. doi: 10.1073/pnas.1112816108

Bdeir, R., Muchero, W., Yordanov, Y., Tuskan, G. A., Busov, V., \& Gailing, O. (2019). Genome-wide association studies of bark texture in Populus trichocarpa. Tree Genetics and Genomes, 15(1). doi: $10.1007 / \mathrm{s} 11295-019-1320-2$

Biswal, A. K., Atmodjo, M. A., Li, M., Baxter, H. L., Yoo, C. G., Pu, Y., ... Mohnen, D. (2018). Sugar release and growth of biofuel crops are improved by downregulation of pectin biosynthesis. Nature Biotechnology, 36(3), 249-257. doi: 10.1038/nbt.4067

Boyle, E. A., Li, Y. I., \& Pritchard, J. K. (2017). An Expanded View of Complex Traits: From Polygenic to Omnigenic. Cell, 169(7), 1177-1186. doi: 10.1016/j.cell.2017.05.038

Broadaway, K. A., Cutler, D. J., Duncan, R., Moore, J. L., Ware, E. B., Jhun, M. A., ... Epstein, M. P. (2016). A Statistical Approach for Testing Cross-Phenotype Effects of Rare Variants. American Journal of Human Genetics, 98(3), 525-540. doi: 10.1016/j.ajhg.2016.01.017

Chhetri, H. B., Macaya-sanz, D., Kainer, D., Biswal, A. K., Evans, L. M., Chen, J., ... Difazio, S. P. (2019). Multitrait genome-wide association analysis of Populus trichocarpa identifies key polymorphisms controlling morphological and physiological traits. New Phytologist, 223, 293-309. doi: 10.1111/nph.15777

Du, Q., Yang, X., Xie, J., Quan, M., Xiao, L., Lu, W., ... Zhang, D. (2019). Time-specific and pleiotropic quantitative trait loci coordinately modulate stem growth in Populus. Plant Biotechnology Journal, 17(3), 608-624. doi: 10.1111/pbi.13002

Eckert, A. J., Wegrzyn, J. L., Pande, B., Jermstad, K. D., Lee, J. M., Liechty, J. D., ... Neale, D. B. (2009). Multilocus patterns of nucleotide diversity and divergence reveal positive selection at candidate genes related to cold hardiness in coastal Douglas fir (Pseudotsuga menziesii var. menziesii). Genetics, 183(1), 289-298. doi: 10.1534/genetics.109.103895

Edel, K. H., Marchadier, E., Brownlee, C., Kudla, J., \& Hetherington, A. M. (2017). The Evolution of 
Calcium-Based Signalling in Plants. Current Biology, 27(13), R667-R679. doi:

10.1016/j.cub.2017.05.020

Evans, L. M., Slavov, G. T., Rodgers-Melnick, E., Martin, J., Ranjan, P., Muchero, W., ... DiFazio, S. P. (2014). Population genomics of Populus trichocarpa identifies signatures of selection and adaptive trait associations. Nature Genetics, 46(10), 1089-1096. doi: 10.1038/ng.3075

Fahrenkrog, A. M., Neves, L. G., Resende, M. F. R., Dervinis, C., Davenport, R., Barbazuk, W. B., \& Kirst, M. (2017). Population genomics of the eastern cottonwood (Populus deltoides). Ecology and Evolution, 7(22), 9426-9440. doi: 10.1002/ece3.3466

Gao, J., \& Lan, T. (2016). Functional characterization of the late embryogenesis abundant (LEA) protein gene family from Pinus tabuliformis (Pinaceae) in Escherichia coli. Scientific Reports, 6(September 2015), 1-10. doi: 10.1038/srep19467

Gonzales-Vigil, E., Hefer, C. A., von Loessl, M. E., La Mantia, J., \& Mansfield, S. D. (2017). Exploiting Natural Variation to Uncover an Alkene Biosynthetic Enzyme in Poplar. The Plant Cell, 29(8), 2000-2015. doi: 10.1105/tpc.17.00338

González-Martínez, S. C., Ersoz, E., Brown, G. R., Wheeler, N. C., \& Neale, D. B. (2006). DNA sequence variation and selection of tag single-nucleotide polymorphisms at candidate genes for drought-stress response in Pinus taeda L. Genetics, 172(3), 1915-1926. doi:

10.1534/genetics.105.047126

Gudesblat, G. E., Schneider-Pizoń, J., Betti, C., Mayerhofer, J., Vanhoutte, I., Van Dongen, W., ... Russinova, E. (2012). SPEECHLESS integrates brassinosteroid and stomata signalling pathways. Nature Cell Biology, 14(5), 548-554. doi: 10.1038/ncb2471

Hackinger, S., \& Zeggini, E. (2017). Statistical methods to detect pleiotropy in human complex traits. Open Biology, 7(11), 170125. doi: 10.1098/rsob.170125

Hansen, S. F., Harholt, J., Oikawa, A., \& Scheller, H. V. (2012). Plant Glycosyltransferases Beyond CAZy: A Perspective on DUF Families. Frontiers in Plant Science, 3(March), 1-10. doi: 10.3389/fpls.2012.00059

Holliday, J. A., Wang, T., \& Aitken, S. (2013). Predicting Adaptive Phenotypes From Multilocus Genotypes in Sitka Spruce (Picea sitchensis) Using Random Forest. G3 Genes Genomes Genetics, 2(9), 1085-1093. doi: 10.1534/g3.112.002733

Ingvarsson, P. K., Hvidsten, T. R., \& Street, N. R. (2016). Towards integration of population and comparative genomics in forest trees. New Phytologist, 212(2), 338-344. doi: 10.1111/nph.14153

Khan, M., Rozhon, W., Bigeard, J., Pflieger, D., Husar, S., Pitzschke, A., ... Poppenberger, B. (2013). Brassinosteroid-regulated GSK3/Shaggy-like kinases phosphorylate mitogen-activated protein (MAP) kinase kinases, which control stomata development in Arabidopsis thaliana. Journal of 
Biological Chemistry, 288(11), 7519-7527. doi: 10.1074/jbc.M112.384453

Lewit-Bentley, A., \& Réty, S. (2000). EF-hand calcium-binding proteins. Current Opinion in Structural Biology, 10(6), 637-643. doi: 10.1016/S0959-440X(00)00142-1

Magwanga, R. O., Lu, P., Kirungu, J. N., Lu, H., Wang, X., Cai, X., ... Liu, F. (2018). Characterization of the late embryogenesis abundant (LEA) proteins family and their role in drought stress tolerance in upland cotton. BMC Genetics, 19(1), 1-31. doi: 10.1186/s12863-017-0596-1

Mckown, A. D., Guy, R. D., Klápště, J., Geraldes, A., Friedmann, M., Cronk, Q. C. B., ... Douglas, C. J. (2014). Geographical and environmental gradients shape phenotypic trait variation and genetic structure in Populus trichocarpa. New Phytologist, 201(4), 1263-1276. doi: 10.1111/nph.12601

McKown, A. D., Guy, R. D., Quamme, L., Klápště, J., La Mantia, J., Constabel, C. P., ... Azam, M. S. (2014). Association genetics, geography and ecophysiology link stomatal patterning in Populus trichocarpa with carbon gain and disease resistance trade-offs. Molecular Ecology, 23(23), 57715790. doi: 10.1111/mec.12969

McKown, A. D., Klápště, J., Guy, R. D., Geraldes, A., Porth, I., Hannemann, J., .. Douglas, C. J. (2014). Genome-wide association implicates numerous genes underlying ecological trait variation in natural populations of Populus trichocarpa. New Phytologist, 203(2), 535-553. doi: 10.1111/nph.12815

Muchero, W., Guo, J., DiFazio, S. P., Chen, J., Ranjan, P., Slavov, G. T., ... Tuskan, G. A. (2015). Highresolution genetic mapping of allelic variants associated with cell wall chemistry in Populus. BMC Genomics, 16, 24. doi: 10.1186/s12864-015-1215-z

Neale, D. B., \& Kremer, A. (2011). Forest tree genomics: growing resources and applications. Nature Reviews. Genetics, 12(2), 111-122. doi: 10.1038/nrg2931

Neale, D. B., \& Savolainen, O. (2004). Association genetics of complex traits in conifers. New Phytologist, 9(7), 325-330. doi: 10.1111/j.1469-8137.2010.03593.x

Porter, H. F., \& O’Reilly, P. F. (2017). Multivariate simulation framework reveals performance of multitrait GWAS methods. Scientific Reports, 7(May 2016), 1-12. doi: 10.1038/srep38837

Porth, I., Klápště, J., McKown, A. D., La Mantia, J., Guy, R. D., Ingvarsson, P. K., ... El-Kassaby, Y. A. (2015). Evolutionary Quantitative Genomics of Populus trichocarpa. PLOS ONE, 10(11), e0142864. doi: 10.1371/journal.pone.0142864

Porth, I., Klápště, J., Skyba, O., Friedmann, M. C., Hannemann, J., Ehlting, J., ... Douglas, C. J. (2013). Network analysis reveals the relationship among wood properties, gene expression levels and genotypes of natural Populus trichocarpa accessions. New Phytologist, 200(3), 727-742. doi: 10.1111/nph.12419

Ranty, B., Aldon, D., Cotelle, V., Galaud, J.-P., Thuleau, P., \& Mazars, C. (2016). Calcium Sensors as Key Hubs in Plant Responses to Biotic and Abiotic Stresses. Frontiers in Plant Science, 7(March), 
1-7. doi: 10.3389/fpls.2016.00327

Ritchie, M. D., Holzinger, E. R., Li, R., Pendergrass, S. A., \& Kim, D. (2015). Methods of integrating data to uncover genotype-phenotype interactions. Nature Reviews Genetics, 16(2), 85-97. doi: $10.1038 / \mathrm{nrg} 3868$

Solovieff, N., Cotsapas, C., Lee, P. H., Purcell, S. M., \& Smoller, J. W. (2013). Pleiotropy in complex traits: Challenges and strategies. Nature Reviews Genetics, 14(7), 483-495. doi: 10.1038/nrg3461

Stephens, M. (2013). A Unified Framework for Association Analysis with Multiple Related Phenotypes. PLoS ONE, 8(7), e65245. doi: 10.1371/journal.pone.0065245

Sterling, J. D., Atmodjo, M. A., Inwood, S. E., Kumar Kolli, V. S., Quigley, H. F., Hahn, M. G., \& Mohnen, D. (2006). Functional identification of an Arabidopsis pectin biosynthetic homogalacturonan galacturonosyltransferase. Proceedings of the National Academy of Sciences, 103(13), 5236-5241. doi: 10.1073/pnas.0600120103

Street, N. R., \& Ingvarsson, P. K. (2011). Association genetics of complex traits in plants. New Phytologist, 189, 909-922. doi: 10.1111/j.1469-8137.2010.03593.x

Visscher, P. M., Wray, N. R., Zhang, Q., Sklar, P., McCarthy, M. I., Brown, M. A., \& Yang, J. (2017). 10 Years of GWAS Discovery: Biology, Function, and Translation. American Journal of Human Genetics, 101(1), 5-22. doi: 10.1016/j.ajhg.2017.06.005

Vogt, T., \& Jones, P. (2000). Glycosyltransferases in plant-natural product synthesis: Characterization of a supergene family. Trends in Plant Science, 5(9), 380-386. doi: 10.1016/S1360-1385(00)01720-9

Voiniciuc, C., Engle, K. A., Günl, M., Dieluweit, S., Schmidt, M. H.-W., Yang, J.-Y., ... Usadel, B. (2018). Identification of Key Enzymes for Pectin Synthesis in Seed Mucilage. Plant Physiology, 178(3), 1045-1064. doi: 10.1104/pp.18.00584

Wegrzyn, J. L., Eckert, A. J., Choi, M., Lee, J. M., Stanton, B. J., Sykes, R., ... Neale, D. B. (2010). Association genetics of traits controlling lignin and cellulose biosynthesis in black cottonwood (Populus trichocarpa, Salicaceae) secondary xylem. New Phytologist, 188(2), 515-532. doi: 10.1111/j.1469-8137.2010.03415.x

Zeng, H., Zhang, Y., Zhang, X., Pi, E., \& Zhu, Y. (2017). Analysis of EF-Hand Proteins in Soybean Genome Suggests Their Potential Roles in Environmental and Nutritional Stress Signaling. Frontiers in Plant Science, 8(May), 1-15. doi: 10.3389/fpls.2017.00877

Zentella, R., Zhang, Z.-L., Park, M., Thomas, S. G., Endo, A., Murase, K., ... Sun, T. (2007). Global Analysis of DELLA Direct Targets in Early Gibberellin Signaling in Arabidopsis. The Plant Cell, 19(10), 3037-3057. doi: 10.1105/tpc.107.054999

Zhang, J., Yang, Y., Zheng, K., Xie, M., Feng, K., Jawdy, S. S., ... Muchero, W. (2018). Genome-wide association studies and expression-based quantitative trait loci analyses reveal roles of HCT2 in 
caffeoylquinic acid biosynthesis and its regulation by defense-responsive transcription factors in Populus. New Phytologist, 220(2), 502-516. doi: 10.1111/nph.15297

Zhou, X., \& Stephens, M. (2012). Genome-wide efficient mixed-model analysis for association studies. Nature Genetics, 44(7), 821-826. doi: 10.1038/ng.2310

Zhou, X., \& Stephens, M. (2014). Efficient multivariate linear mixed model algorithms for genome- wide association studies. Nature Genetics, 11(4), 407-411. doi: 10.1038/nmeth.2848

Zhu, J. K. (2016). Abiotic Stress Signaling and Responses in Plants. Cell, 167(2), 313-324. doi: 10.1016/j.cell.2016.08.029 